\title{
An experimental investigation into the feasibility of MIMO techniques within the HF band
}

Thesis submitted to the Faculty of Science, University of Leicester in candidature for the degree of Doctor of Philosophy

\section{By}

\author{
Nasir Mehmood Abbasi \\ Department of Engineering \\ University of Leicester
}

August 2011 


\title{
Nasir Mehmood Abbasi
}

An experimental investigation into the feasibility of MIMO techniques within the HF band

\begin{abstract}
MIMO research so far has primarily focussed on the VHF-SHF frequency bands with an aim to increase the data rates achieved over a limited spectrum. MIMO requires a rich scattering environment in order to create parallel spatial data pipes between antennas at the transmitter and the receiver. The HF band is also affected by the multipath effects through reflections in the different layers of the ionosphere and hence is a suitable candidate for MIMO.
\end{abstract}

A $255 \mathrm{~km}$ radio link was established between Durham and Leicester in UK to make measurements using different types of antenna arrays in the HF band. Both spaced and collocated antenna arrays were used in various campaigns over the period of two years between September 2007 and August 2009. The correlation between the signals from antennas in a spaced array was found to depend not only on the separation distance but also on the orientation of the antennas in the array. The correlation between the antenna elements in the collocated arrays was dependent on the polarization and the far field patterns (both magnitude and phase). The capacity estimates derived from the channel matrix obtained using the Fourier transform of the received signal verified the correlation coefficient results.

NEC-2 software was used to analyze the response of the antennas in the array. It was determined that antenna modelling could be used to design small, collocated antenna arrays for HF-MIMO application.

This research may act as the foundation stone on which the future endeavours into the application of MIMO techniques in the HF band could be built. 
Acknowledgement

It is a pleasure for me to thank the people who contributed towards this research. My gratitude goes to Professor Mike Warrington who supervised this research and was always willing to encourage and support me in addition to the invaluable input and suggestions. Dr. David Siddle as co-supervisor was always there to listen and advise, no matter how busy he was. I would also like to acknowledge Dr. Salil Gunashekar for his forthcoming support and co-operation throughout this project.

I am indebted to my wife who supported me all along and showed great patience. She shielded me from many day to day worries and allowed me the time and space to concentrate on my research.

I would not want to miss out on dedication to my deceased parents whose love and affection has always been a source of strength for me and their memory is still the most cherished asset of my life.

Lastly but most importantly thanks to the God Almighty who is the supreme architect and makes things possible for human beings. 


\section{Contents}

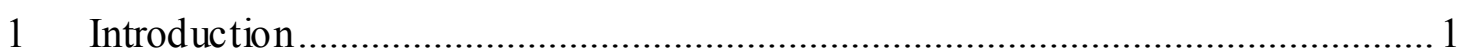

1.1 Use of multiple antennas in a multipath environment.................................... 3

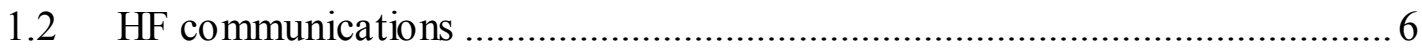

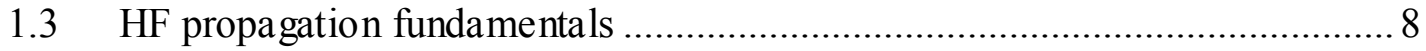

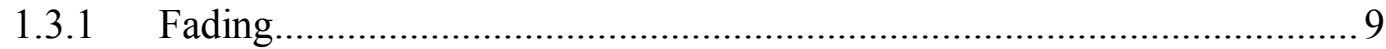

$1.4 \quad$ Ionosondes and oblique propagation........................................................ 10

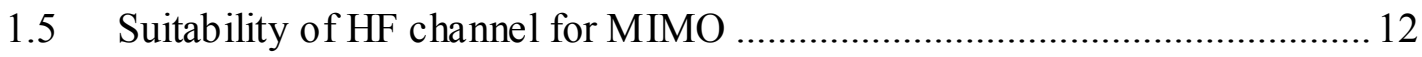

1.6 Important contributions of this research................................................... 13

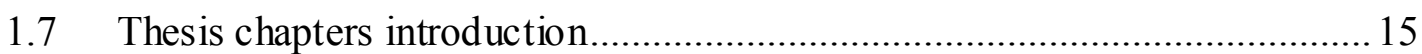

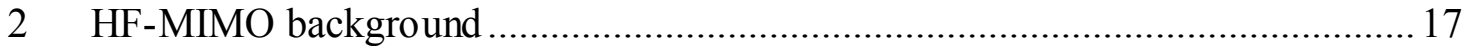

2.1 Theoretical Framework for MIMO Channel Capacity................................. 17

2.2 Spatial Multiplexing Algorithm development............................................. 19

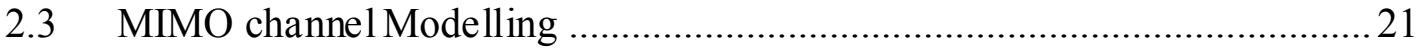

2.3.1 Gesbert's channel model................................................................ 21

2.3.2 Experimental characterization of MIMO channel .............................. 22

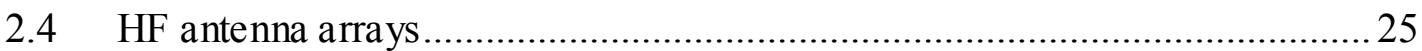

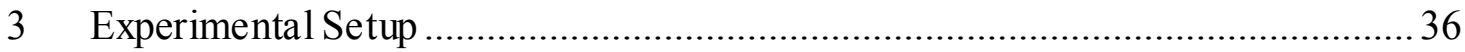

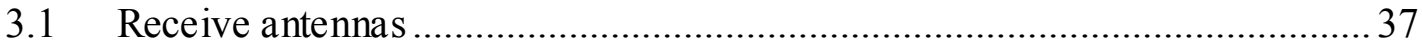

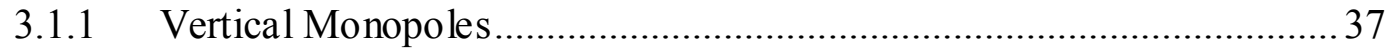

3.1.2 Crossed wire Inverted V antennas .................................................... 40

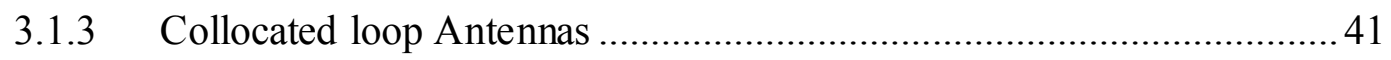

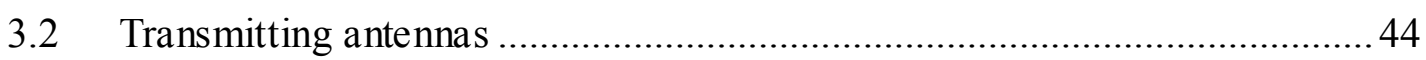

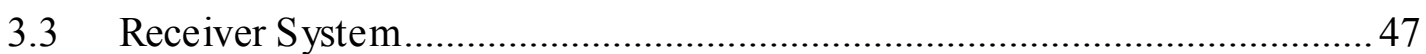

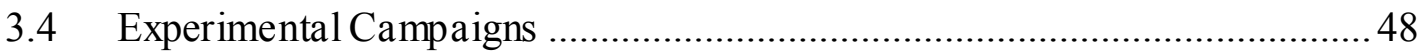

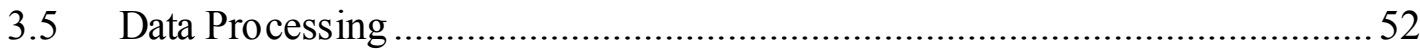

4 Measurements using spaced and collocated antenna arrays in HF-MIMO ..........55

4.1 Correlation measurements using the L-shaped spaced array .........................55

4.1.1 A comparison of separation distance and orientation dependent effects on correlation .55

4.1.2 A comparison of separation distance effects on correlation .................... 60

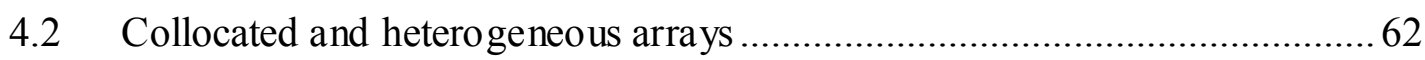

4.2.1 Experiment with the crossed wire inverted $\mathrm{V}$ antennas and the

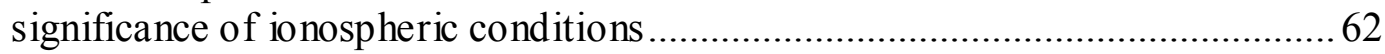

4.2.2 Comparison of spaced arrays and the collocated array in 4x4 MIMO ... 69

4.2.3 Comparison of crossed wire array with smaller loop array and monopole

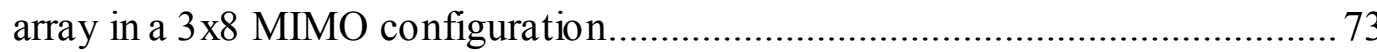


4.2.4 Comparison of large collocated array and small collocated active array in $4 \times 8$ MIMO configuration

4.2.5 Comparison of large collocated array (crossed wires) and small collocated array (active dipo les+monopole) in 2x8 MIMO configuration

4.2.6 Comparison of large collocated array and small collocated Giselle array in $2 \times 8$ MIMO configuration

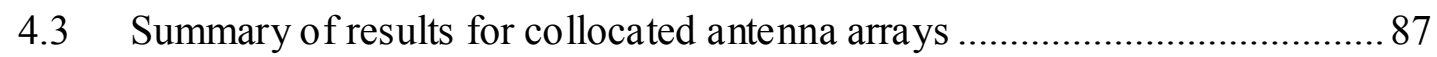

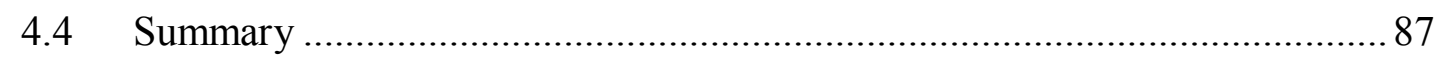

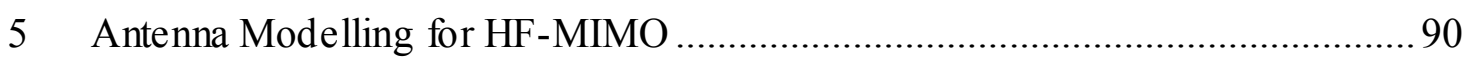

5.1 Antenna Modelling with Numerical Electromagnetic Code (NEC) .............. 90

5.1.1 Modelling guidelines for NEC ........................................................ 91

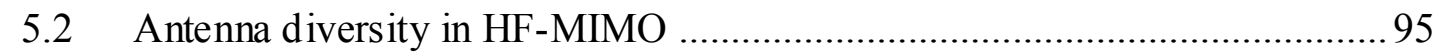

5.3 Modelling of collocated HF-MIMO antennas .............................................. 96

5.4 Modelling results for crossed wire inverted $V$ antennas .............................. 98

5.4.1 Comparison of simulations with experiments ...................................... 101

5.5 Modelling results for Giselle collocated loop antennas .............................. 104

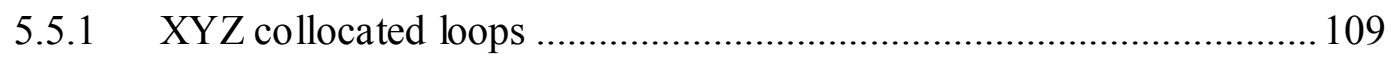

5.5.2 Modelling results for crossed dipoles with a vertical antenna .............. 115

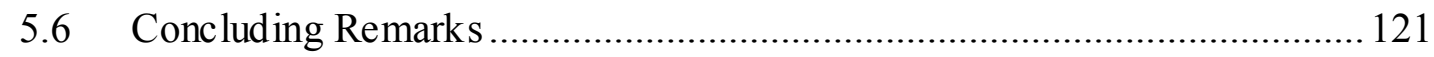

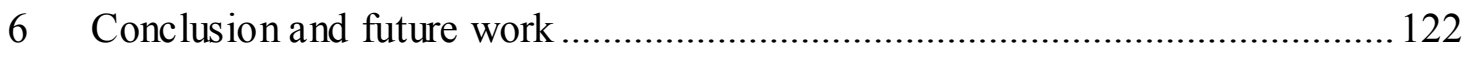

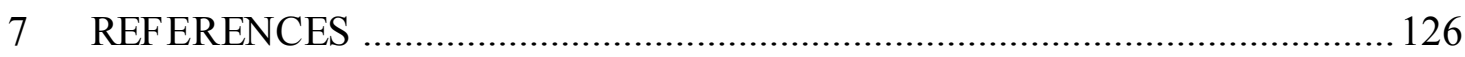

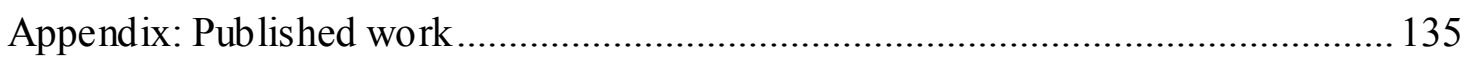




\section{Introduction}

Wireless communication technologies have made huge progress in the recent decades. Data rates that can now be achieved wirelessly have increased tremendously; ho wever the applications are also becoming more and more data intensive putting a strain on the limited bandwidth resource. Thus new ways to utilize the spectrum efficiently have to be explored. Multiple input multiple output (MIMO) is one such method which uses more than one antenna at both transmit and receive ends of the communication link. MIMO introduces space as another variable that can be utilized to introduce additional degree of freedom within the same bandwidth.

A simple diagram depicting a MIMO system using $\mathrm{M}$ transmit and $\mathrm{N}$ receive antennas is shown in the Figure 1.1.

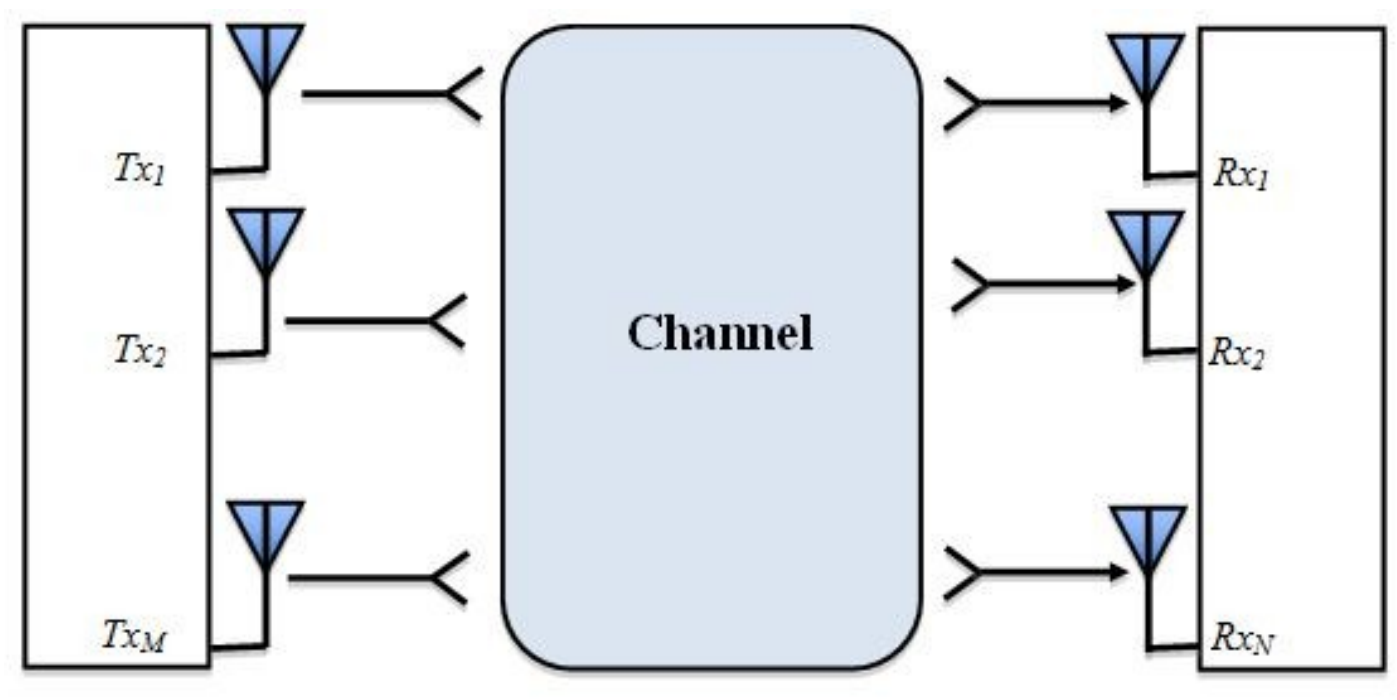

Figure 1.1 MIMO system

MIMO offers a number of advantages over the traditional single input single output (SISO) systems. These advantages stem from the fact that signals from different input antennas traverse different propagation paths provided by the multipath channel. Multiple antennas at transmit and receive ends of the communication link along with suitable coding and signal processing has been shown to improve the reliability of 
communications or to increase the data throughput. These two are not mutually exclusive however and coding techniques exist which allow for both advantages to be gained simultaneously [Bohnke et al, 2004]. The benefits of employing MIMO can be categorized as follows [Paulraj et al., 2003].

\section{1) Array Gain}

It is the power gain or the increase in SNR. Array gain can be achieved using single input multiple output (SIMO), MIMO or multiple input single output (MISO) systems. Channel knowled ge is required at the receiver in SIMO and at the transmitter in MISO systems to exploit array gain.

\section{2) Diversity Gain}

Diversity gain helps overcome the effects of multipath fading by increasing the signal to interference ratio. A combining technique at the receiver is used to optimize this gain. Diversity antennas at receiver have been employed for quite some time. If the transmit-receive antenna pairs undergo independent channel fades then the diversity order is the product of number of transmit and receive antennas. Diversity gain can also be achieved in MISO and MIMO systems using space time coding.

\section{3) Spatial Multiplexing Gain}

If the channel is rich in multipath, independent streams of communication can be used between antenna pairs. Data can be multiplexed and sent over these channels to achieve a high capacity. Ideally this capacity increase is proportional to the minimum of the number of transmit and receive antennas.

4) Interference Reduction

MIMO can be used to mitigate the effects of co-channel interference to improve the capacity. Multiple antennas can be used to discriminate between the desired signal and 
the interferer and thus filter it out. Interference reduction can be implemented at both the transmitter and the receiver.

Wireless communication has to deal with the effects introduced by the channel. These include signal degradation due to path loss and the arrival of copies of the signals at the receiver through different routes. Multipath propagation between the transmitter and receiver is possible due to the presence of obstacles which cause reflections, refractions and scattering. Such phenomenon redirects and spread the signal energy. This results in copies of the signal which undergo different amplitude and phase variations and arrive at the receiver with different path delays. These copies combine at the receiver constructively or destructively depending upon their phase and result in deep nulls in the received signal. This phenomenon is called multipath fading. The variations in the amplitude of the signal at the receiver do not allow a high data rate to be maintained. Multipath propagation also causes inter symbol interference (ISI) when the delay between the two copies is comparable with the symbol length. The symbol periods get overlapped necessitating a lowering of data rate in order to maintain the integrity of transmission. Different methods have been used to overcome the deleterious effects of multipath propagation which involve using more antennas at the receiver e.g. diversity, using better signal processing techniques like the rake receivers and by using multiplexing algorithms like the OFDM.

\subsection{Use of multiple antennas in a multipath environment}

MIMO technology is distinguished by the use of more than one antenna at the transmitter and the receiver. It is based on the premise that the multipath environment introduces replicas of the signal which arrive at the receiver with different amplitude, phase and angle of arrival. Thus if more antennas are introduced, each one of them 
might be able to receive signals which come from a different path and are thus ind ependent. Thus MIMO envisages turning a phenomenon that was considered to be a drawback into something advantageous; instead of suppressing the copies of the signal it utilizes these as additional channels of propagation. Classic implementation of MIMO requires a Rayleigh channel undergoing independent fades. Real wireless channels also fulfil the basic condition for the application of MIMO most of the times.

To exploit a multipath environment, antenna type, orientation and placement is very important. Thus any number of similar antennas placed very close to each other with same orientation would not prove beneficial. Each antenna in a MIMO configuration must be able to receive components of the multipath signals which are ind ependent. In other words signals received on the antennas must not be correlated as it is only through the signals being uncorrelated that more channels can be set-up at the same carrier frequency. Classically, in order to achieve decorrelation spaced antennas have been used in diversity applications. Recent developments in MIMO use collocated antennas with pattern and polarization diversity to overcome space constraints and some of such antennas designed for MIMO in the high frequency (HF) band form part of this thesis. The main parameters for MIMO antennas performance are the correlation, mutual coupling and total active reflection coefficient (TARC) [Sung Ho Chae et al., 2007]. Correlation determines the likeness of one signal with the other. Mutual coupling is the effect of the EM fields of one antenna on the other antenna and decreases the efficiency of the closely spaced antennas. TARC is the ratio of the incident power to the power actually being radiated by the antennas.

The drawbacks of MIMO include the additional space requirements and the signal processing complexity introduced. Space time codes are used if the same information is to be transmitted on all antennas to improve reliability while spatial 
multiplexing algorithms use each transmit-receive pair as an independent channel and carry different user data.

The different ways to introduce decorrelation between the MIMO antennas are: 1) Space diversity: Decorrelation is achieved by placing sensors at a distance (measured in terms of wavelength) from one another. In order to achieve significant decorrelation a minimum spacing of $\lambda / 2$ is recommended [Jakes, 1974].

2) Pattern diversity: This kind of diversity is achieved using antennas with varying radiation patterns. The main beam of the MIMO antennas is directed at different multipath components to receive de-correlated signals [Liang Dong et al., 2002] Pattern diversity can be used to achieve significant decorrelation for high capacity gains.

3) Polarization diversity: Polarization diversity means using antennas with different polarizations. Polarization mismatch can cause a 10-20 dB loss in the received signal power. Such losses can be reduced significantly using polarization diversity which can lead to capacity gains [Andrews et al, 2001]. In the case of HF the signals are elliptically polarized and divided into two separate modes distinguished by the direction of their rotation in the clockwise and anti-clockwise direction. The ability to separate these modes at the receiver could result in the diversity gain in heterogonous arrays.

In MIMO applications to achieve capacity gains pattern and polarization diversity are normally applied simultaneously. It has been shown that application of polarization and pattern diversity increases the decorrelation and hence the capacity. Large gains up to $12 \mathrm{~dB}$ can be achieved using spatial, polarization and pattern diversity at 2.05 GHz carrier frequency [Dietrich et al., 2001]. 


\section{$1.2 \mathrm{HF}$ communications}

The ionosphere is a region extending from $50 \mathrm{~km}$ to $500 \mathrm{~km}$ from Earth's surface. It consists of ionized particles, which can cause radio waves to refract [Davies, 1990]. Ionosphere is formed by the ultraviolet (UV) radiation from the sun which breaks the structure of the stable gases in the region into electrons and positive ions.

The ionosphere is very variable in terms of its composition. The variations are both temporal and geographical. Temporal changes include the difference in the ionospheric structure during the day and the night, as well as seasonal changes. The position of the sun which depends on the 11 year periodic solar cycle is another factor which determines the state of the ionosphere. Solar flares resulting from an increase in $\mathrm{X}$-ray and ultraviolet rays cause an increase in the total electron content (TEC) in the E and $\mathrm{F}$ layers. The composition of ionosphere is not uniform around the globe and there are significant changes at different latitude. Geomagnetic storms are also linked with the changes in the ionosphere.

The ionosphere is generally classified vertically into three layers.

D layer

It is the lowest layer of the ionosphere extending from $50-90 \mathrm{~km}$ above the surface of the Earth. High rate of recombination renders this layer to be very lightly ionized. It absorbs frequencies in the lower end of the HF band and thus obstructs communication. It is present during day time only.

E layer

This is the middle layer ranging in height from $90-140 \mathrm{~km}$ in the atmosphere and is highly variable in space and time. It is important for reflecting some signals in the lower HF band. The ionization of this layer diminishes significantly during the night. It contains more intense patches of ionization called sporadic E layer. 
F layer

The $\mathrm{F}$ layer is the most important layer for HF communic ations with an approximate elevation of $140-400 \mathrm{~km}$. It has the greatest electron density and is predominantly responsible for $\mathrm{HF}$ communication at night along with the sporadic $\mathrm{E}$ layer. $\mathrm{F}$ layer is divided into F1 and F2 regions. F1 is the lower part of the F region which is present only during the day time and merges with the F2 layer during the night.

Figure 1.2 shows the plasma density of the different layers of the ionosphere compared to the height on the left side. It can be seen that the electron density profile is very much different during the day and night time. On the right the vertical temperature profile and the processes are given.

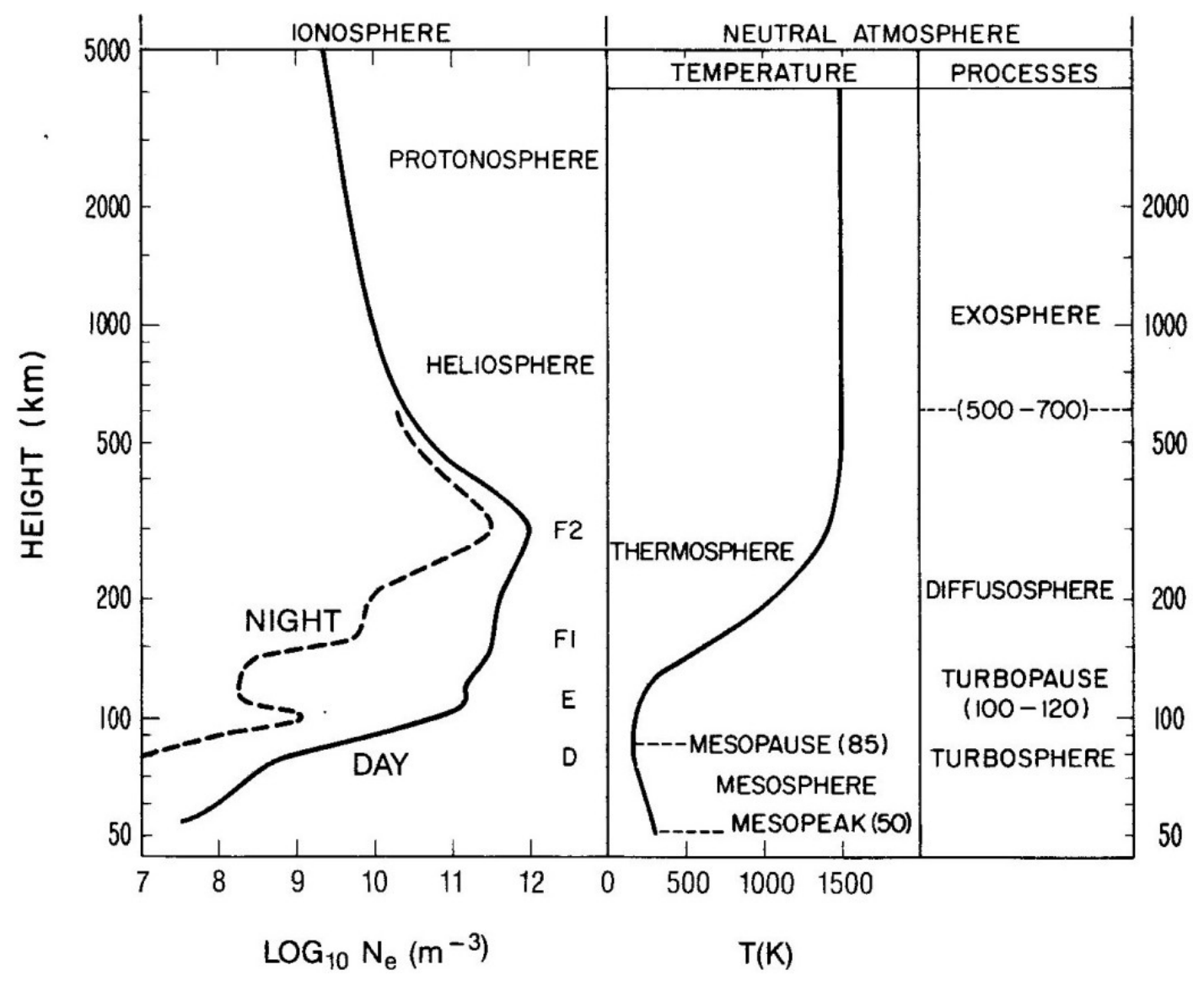

Figure 1.2 Plasma density in relation to height (taken from Ionos pheric Radio by K. Davies) 


\subsection{HF propagation fundamentals}

HF communication can take place through ground waves, direct line of sight or the sky wave. However sky wave communication is most important for long distance communication. An important decision in HF communications is the selection of the frequency of transmission. The optimum working frequency is selected from a range of frequencies between the lowest useable frequency (LUF) and the maximum useable frequency (MUF). LUF and MUF depend on ionospheric conditions as well as the link geometry. The wave incident on the ionosphere may be split up in different parts going along different paths in the ionosphere. These different paths are called modes. The propagation paths are ascribed by 3 characters with the first character for the number of hops to the receiver and the other two for the ionospheric layer used e.g. 1F1 would be the one hop path reflecting from F1 layer and $2 \mathrm{~F} 2$ would be the 2 hop path reflecting from the F2 layer. This is shown in the Figure 1.3. There may also be present other complex modes like the chordal modes and modes due to the phenomenon of ducting.

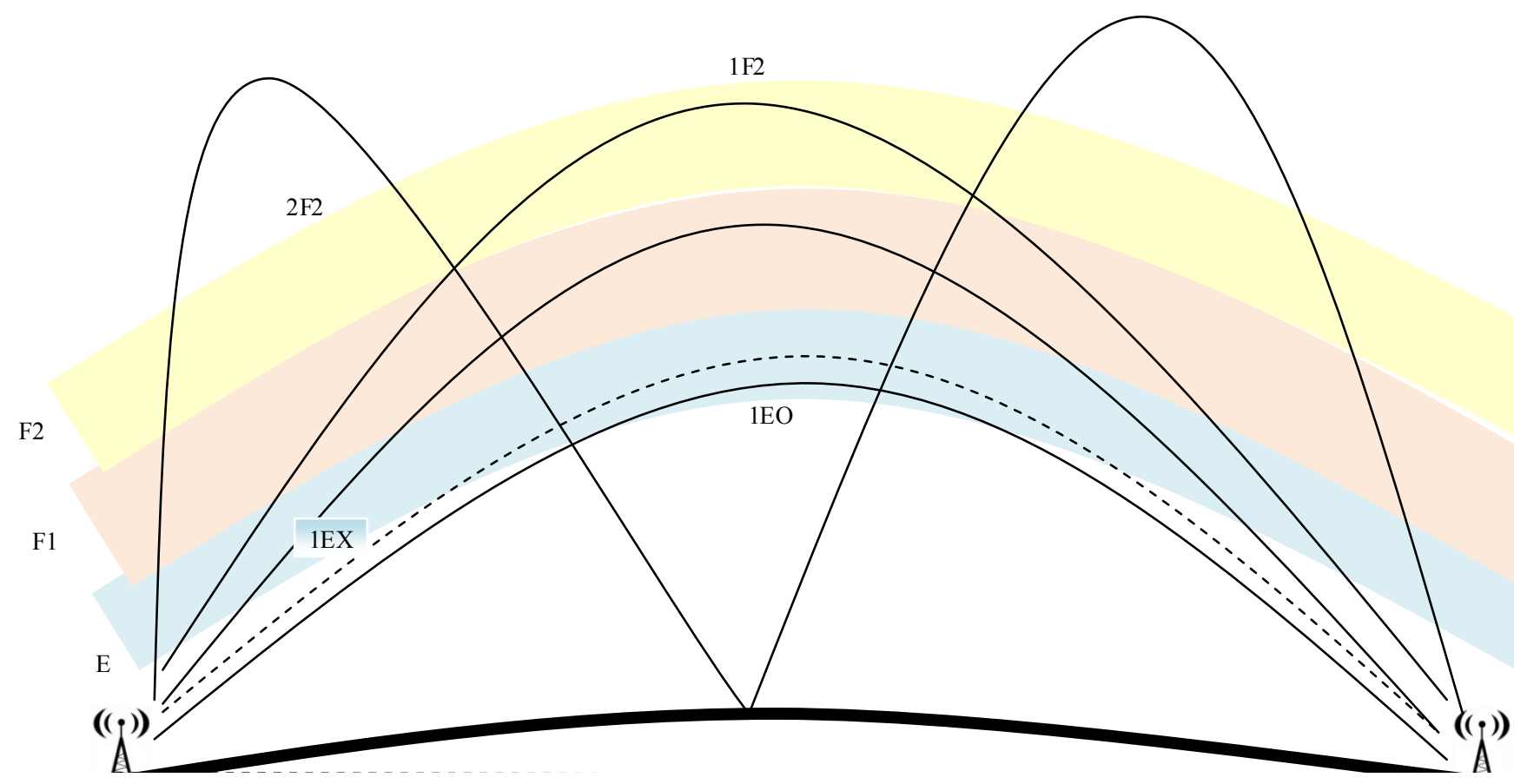

Figure 1.3 Ionos pheric communication through different layers 


\subsubsection{Fading}

Fading causes rapid changes in the amplitude of the received signal. In the ionosphere it can be of the following types:

1) Multipath Fading

The multipath in HF is generated by the ionosphere's ability to create multiple copies of the signal reflected by the different layers of the ionosphere. The replicas of signal with different amplitudes and phases combine at the receiver to cause fading. Two copies of the signal with a group path which differs by $n \lambda / 2$ (where $\mathrm{n}$ is odd) will result in a complete cancellation of the signal. These deep nulls would disrupt the communication completely. Multipath fading is at its worst between signals from $1 \mathrm{~F}$ and $2 \mathrm{~F}$ modes or $2 \mathrm{E}$ and $1 \mathrm{~F}$ modes [McNamara, 1991]. It causes the signal level to drop at the receiver and does not allow a constant data rate to be maintained.

2) Polarization Fading

The ionosphere due to its anisotropic nature (caused due to Earth's magnetic field) splits the HF signal into magneto ionic modes. These modes are the ordinary mode and the extraordinary mode. These waves are elliptically polarized generally, changing to linear polarizations nearer to the equator and circular polarization in lower polar regions. These elliptically polarized waves rotate in opposite directions and propagate independently of each other. These modes can add to form a linearly polarized wave if both have the same amplitude and phase. This does not happen however as $\mathrm{O}$ and $\mathrm{X}$ modes have different phase velocities resulting in phase difference which increases as the waves propagate through the ionosphere. The two modes cause fading at the received antenna. Polarization fading can be overcome in two extreme cases of linear and circular polarizations by using orthogonal sensors [Davies, 1990]. 


\section{4 lonosondes and oblique propagation}

Ionosondes are used to monitor the ionosphere for communications and research purposes. An Ionosonde is a type of radar that sweeps through a range of frequencies. As the frequency increases so does the delay till a point is reached when the waves penetrate the ionosphere never to return back. The frequency for which this happens in case of vertically transmitted signal is called the critical frequency. The result obtained from an ionosonde can be represented in the form of an ionogram which is a graph of virtual height $v$ frequency. A sample ionogram is shown in the Figure 1.4.

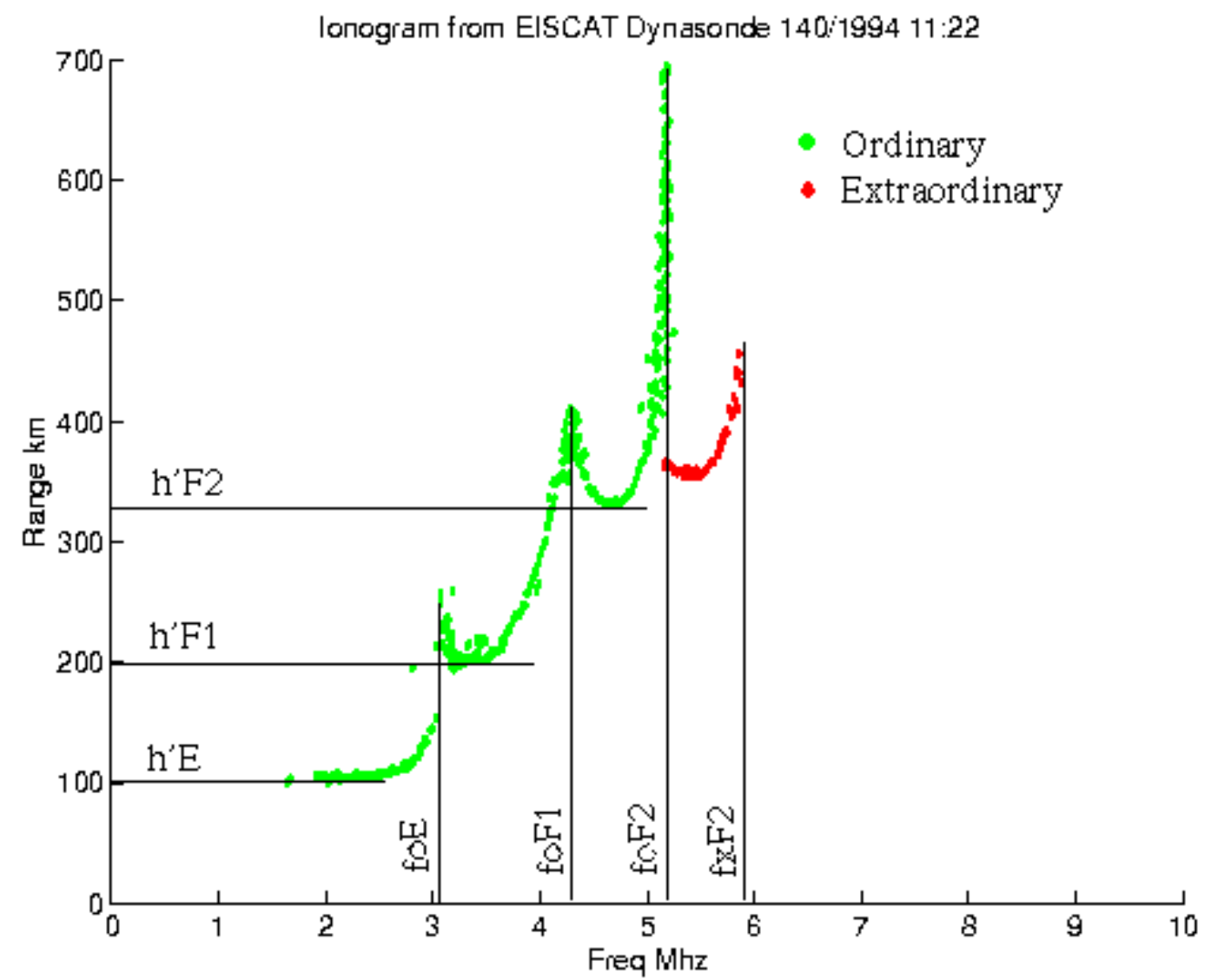

Figure 1.4 Sample ionogram taken from UKSSDC website

Since the reflection in the ionosphere is based on the difference in refractive indices of the layers, the angle at which the ray is incident on the ionosphere 
determines what frequency is to be used. The basic relationship between the oblique and vertical frequencies is given by the secant law:

$$
f_{o}=f_{v} \sec \varphi_{0}
$$

Where $f_{o}$ is the oblique frequency for the angle $\phi_{o}$ and $f_{v}$ is the frequency for the vertical wave. For these equivalent frequencies the virtual height of reflection for the vertical frequency is equal to the height of the oblique frequency.

Martyn's equivalent path theorem relates the virtual height of reflection for the oblique and vertical frequency when these are reflected from the same real height:

$$
P\left(f_{o}\right)=2 h^{\prime}\left(f_{v}\right) \sec \varphi_{0}
$$

Where $P\left(f_{o}\right)$ is the oblique path length of the signal and $h^{\prime}\left(f_{v}\right)$ is the virtual height. Ionosondes are basically characterized into vertical and oblique. Oblique sounders mimic the real communication between the transmitter and the receiver and give a more accurate description of the ionospheric channel which is horizontally variable in nature.

Vertical ionosondes are more prevalent because of ease of use and due to the fact that their results can be converted into oblique ionograms for small distances by making some assumptions. Ionograms give a very useful insight into the status of the ionosphere and its behaviour towards different frequencies at that time. The layers involved in communication, modes and the group heights can then be determined. The virtual height as a function of vertical frequency can be determined from an ionogram which is used to determine the oblique frequency of transmission. The relationship between the secant of the angle of transmission and the virtual height is called a transmission curve [Davies, 1990]. The graphs of such curves are superimposed on 
ionograms to obtain some useful results about a particular transmit-receive system. Transmission curves are drawn using the formula:

$$
f_{o}=f_{v} \sec \phi_{o}=f_{v}\left\{1+\left(\frac{D}{2 h^{\prime}}\right)^{2}\right\}^{1 / 2}
$$

Where $\mathrm{D}$ is the distance between the transmitter and the receiver and $h^{\prime}$ is the virtual height. These curves intersect the ionogram at certain points which determine the virtual height of the layers involved in the communication. The highest useable frequency can be determined by increasing the oblique frequency and plotting transmission curves on the ionogram until the curve completely misses the ionogram data.

Ionosondes can be used to determine the critical frequency, height, polarization, Doppler shift and direction of arrival for a certain frequency. Other methods to study the ionosphere are incoherent scattering and observing it from top using space rockets and spacecrafts.

\subsection{Suitability of HF channel for MIMO}

The basic requirement for the application of MIMO is the existence of multipath effects in a channel. In the case of HF band the number of multipath components originating between a transmitter and receiver depend on the operating frequency, link geometry, time of day, season, geomagnetic activity and sunspot number.

In the case of indoor wireless and cellular communications where most of the MIMO research has focussed the multipath signals arrive from different azimuth angles while the elevation angle stays approximately the same. For signals propagated through the ionosphere in the mid-latitudes the modes arrive at the receiver from different elevation angles but the azimuthal direction stays the same. 
HF wavelengths lie between 10 and 100 metres. The antenna size for these wavelengths is huge compared to the other bands where MIMO has been used. Using spaced antenna arrays 10 s of metre apart is impractical in many HF applications thus making the use of collocated antennas necessary in HF-MIMO applications. This thesis deals with the issue of collocated antennas in the HF band for MIMO applications. Also an attempt is made to further reduce the antenna size by utilizing electrically small (in terms of wavelength) antennas with active electronics.

\subsection{Important contributions of this research}

This research was aimed at the implementation of a MIMO system in the HF band. It entailed selection of transmit and receive sites, procurement of multi-channel transmitter and receiver, design/purchase of transmit/receive antennas, experimental set-up configuration, data gathering, analysis and the modelling of antennas in software. Since the project was a joint venture between University of Leicester and Durham University, various tasks were distributed among team members at both locations. Transmitter site was chosen to be located at Durham and the receive site in Bruntingthorpe, Leicester. Most of the antennas used in the experiments were built at Durham University. The author formed part of the team at the receiver site in Leic ester.

Data gathered during the experiments was read into MATLAB for analysis. The correlation coefficient between the various antenna pairs were calculated and put in a format ready for presentation. The scope of this study was quite large considering the fact that it was the first true experimental implementation of MIMO in the HF band. Experimental configurations were not limited to employing different levels of space diversity. A series of experiments were carried out in which different types of antennas 
were used simultaneously in the MIMO configuration as well as electric and magnetic sense collocated antennas which provided polarization and pattern diversity. Thus the traditional spaced arrays, which can only be used in limited applications due to the large areas required to accommodate them were replaced with collocated arrays and a further reduction in size (up to $1 \mathrm{~m}^{2}$ ) was achieved using active antenna elements at Durham University. These small collocated arrays gave comparable performance and were found to give low correlation coefficients.

The envelope correlation coefficient represents the degree of multipath available in the channel and the ability of the different antenna pairs to make use of the independent modes present. Since reduction in envelope correlation coefficient is necessary for MIMO implementation, useful results were obtained by comparing antenna pairs in different combinations of both homogenous and heterogeneous arrays under the same frequency and environmental conditions. These results are novel both in terms of being the first ever experimental computations of the HF-MIMO systems and the usage of the space, polarization and pattern diversity employing a wide range of different kinds of antennas individually and in the collocated fashion. Capacity estimates from the available data were also computed which allows for the measurable range of improvements using MIMO arrays of different order. The correlation coefficient as well as the capacity estimates indicated considerable improvement over the SISO Shannon capacity. These results are very significant as HF radio band is unable to support high data rates required in some modern applications and MIMO implementation in this band can increase the data rates available and provide a low cost and easy to deploy alternative providing global coverage.

An attempt was made to model HF-MIMO antenna arrays using software in the second part of the project. MIMO studies carried out so far describe the role of 
separation distance, polarization and magnitude pattern for providing diversity in an array. In this study phase patterns were identified as a factor that could lead to diversity in antenna arrays. Orthogonal phase patterns could make use of the different modes of the available multipath and cause diversity just like the antennas with their radiation patterns directed towards different multipath components. Thus antennas could be modelled with phase pattern diversity which could produce predictable results in experimentation and ultimately in real communication systems. The study of the factors that result in decorrelation can also lead to a comparison of the antenna arrays while modelling and the prediction of their behaviour under various channel conditions. This will be a great improvement to the current more qualitative analysis of the effects of space, polarization and pattern diversity.

The thesis as a whole provides a basic framework on which the study of HFMIMO systems can be built. Though a first step in this direction, it clearly indicates the feasibility and the potential improvements which can be accrued by employing MIMO in the HF band using well designed, purpose built antennas arrays.

\subsection{Thes is chapters introduction}

The first chapter of this thesis is the introduction to MIMO and the basic principles of HF propagation. It also makes a case for the suitability of applying MIMO techniques in the HF band. The second chapter contains the detailed theoretical background and literature review of MIMO. In addition the research work carried out using HF antenna arrays in beamforming and diversity applications are also discussed.

The experimental arrangement for carrying out HF-MIMO campaigns is discussed in Chapter 3. It particularly describes the construction of antennas used, the transmitting/receiving equipment and other necessary details about the data collection 
procedure. The analysis of the data collected during various campaigns is presented in Chapter 4 which compares spaced and collocated arrays in terms of inter-element correlation and capacity under varying ionospheric conditions.

Chapter 5 has details about the modelling of HF antenna arrays for MIMO applications and the last chapter is a summary of this study along with ideas for future research. 


\section{HF-MIMO background}

MIMO has been shown to increase the capacity of a wireless communication system under multipath fading conditions [Foschini and Gans, 1998]. It utilizes space as another degree of freedom to increase the data rate without requiring any additional bandwidth. Under ideal conditions capacity of the system increases proportional to the number of antenna pairs used as long as enough independent multipath components are available [Telatar, 1995].

\subsection{Theoretical framework for MIMO channel capacity}

MIMO variants have been around for quite a while, though the coinage of the term is relatively new. Diversity antennas both transmit and receive has been used extensively in the past to improve the radio system performance. Phased array beamformers are used to cancel out noise and interference by creating beams and nulls in the radiation pattern. The same concept of beamforming is a precursor to precoding in MIMO. The idea of using multiple antennas to increase the efficiency of radio link goes back to early 70s when some promising results were published [Kaye and George, 1970]. Winters in his work at Bell labs showed that an increase in capacity can be achieved by using multiple antennas in a Rayleigh fading environment and that parallel data streams could be created between each transmit-receive pair using the same frequency bandwidth [Winters, 1987]. This marked an important milestone in MIMO research. Paulraj and Kailath proposed the concept of Spatial Multiplexing using MIMO [Paulraj and Kailath, 1994]. Emre Telatar [1995] gave the first rigorous treatment to finding the theoretical upper bound for the MIMO system and Foshini [1996] came up with the first spatial multiplexing algorithm for MIMO systems. These early 
developments led to a huge research activity in the field which is mature now and the new wireless standards are incorporating MIMO e.g. WLAN 802.11n standard.

Telatar [1995] determined the capacity for a multiple transmit/receive array system over a Gaussian channel under conditions of the channel matrix being deterministic, random according to probability distributions and random but fixed once chosen. The fading between each transmit-receive pair is considered independent and that the channel is known at the receiver. These conditions allow a mathematical expression to be calculated expressing the upper bound for the capacity of a MIMO channel. The mathematical realization of capacity is achieved by maximizing the mutual information in case of memoryless channels and when the channel is ergodic. In case of non-ergodic channels the capacity is always zero irrespective of the data rate and code length. In this case the outage probabilities can be given for data rates that could be supported. Thus minimum error probability could be maintained using appropriate codes which satis fy the power constraints. Telatar [1995] also discussed the use of equal power sharing for maximizing data rates if the channel is not known at the transmitter. In the case of channel state information known at the transmitter linear increase in capacity (with the increase in number of transmit-receive antennas) will results in case of independent Rayleigh fading channels.

Telatar's seminal work was followed by a major contribution from Foschini. He along with Gans showed that a linear increase in capacity was possible at high SNR when independent Rayleigh channels existed between multi element arrays [Foschini and Gans, 1998]. They used chi square variates for Rayleigh channel representation and derived mathematical expressions for SIMO, MISO and MIMO systems. Independent identically distributed (i.i.d.) Rayleigh fading channels were considered with the assumption that channel state information (CSI) is known at the receiver. 
Foschini and Gans built their argument as an extension to the receive diversity and plotted complementary cumulative distribution function (CCDF) curves to show the capacity gains for equal number of transmit and receive antennas.

The highest data rate supported by the channel with arbitrarily small probability of error is called channel capacity. It is defined as:

$$
C=\log _{2}\left(\operatorname{det}\left(\frac{\rho}{n_{T}} H H^{H}+I_{n_{R}}\right)\right) b p s / H z
$$

Where $C$ is the capacity, $\rho$ is the average SNR, $n_{T}$ is the number of transmitters,,$n_{R}$ is the number of receivers $H$ is the channel matrix, $H^{H}$ is the conjugate-transpose of the channel matrix and $I_{n R}$ is $n_{R} \mathrm{x} n_{T}$ identity matrix .

In deriving the capacity of MIMO channel following assumptions have been made:

a) Channel is Rayleigh fading i.i.d and narrowband (flat fading).

b) Noise affecting the channel is AWGN

c) Power is distributed equally on all transmitters

d) Channel state information (CSI) is known at the receiver only

When $n_{T}$ is large and the channel matrix is orthogonal i.e. $H H^{H}=H^{H} H=I_{M}$

$$
C=n_{R} \log _{2}(1+\rho)
$$

Equation 2.2 represents the upper bound of channel capacity under the ideal assumptions of uncorrelated transmit signals and orthogonal channel matrix. If the channel is sufficiently de-correlated MIMO channels can support higher capacities.

\subsection{Spatial multiplexing algorithm development}

To translate the capacity gains predicted theoretically, practical algorithms need to be developed. Paulraj et al. [1994] had proposed and patented a technique to split high data rate signal into low data rate signals to be transmitted on multiple spaced 
antennas. The system was called distributed-transmit directional-receive (DTDR) system. This used spaced antennas to transmit low data rate signals and directional receivers to form transmit-receive pairs. The direction of arrival would differentiate different transmit antennas and would give rise to the degrees of freedom for the wireless channel. These received signals are demodulated and combined to create the original high data rate signal.

Foschini [1996] presented a spatial multiplexing scheme called the Bell Labs Layered Space Time Architecture (BLAST) to make use of the multipath modes and to lessen the inter coupling between these modes [Foschini, 1996]. This basically is the design of $\mathrm{n}$ (number of transit-receive antenna pairs) modulator/codec blocks called layers. Each bit stream periodically cycles all antennas with a dwell time of $\tau$ which ensures that the data rates on each path stay fairly constant. At the receiver the channel is known using training sequences. The layering of the architecture means that for the first receive antenna, n-1 interferers exist which have to be nulled out. This number decreases by one for each stage and for the last antenna there are no interferers. This algorithm gives the performance equating to the lower bound on the capacity of a theoretical MIMO system. The first implementation of BLAST was tested in the Bell labs and named as V-BLAST [Golden et al., 1999].

Alamouti introduced the Space Time Code for using transmit diversity on the pattern of classical maximal ratio receive combining (MRRC) scheme [Alamouti, 1998]. He showed the coding for a $2 \times 1$ transmit-receive system which could be extended to multiple transmit and receive antennas. The basic requirement for the successful implementation of this scheme is that the antennas on both sides are sufficiently de-correlated $(<0.7$ correlation). Tarokh et al. [1999] presented orthogonal codes to benefit from the diversity gains. They used the mathematical theory of 
generalized orthogonal designs to develop coding schemes which are not constrained by the number of transmit antennas and require linear processing for detection at the receiver. Space time codes improve the reliability of transmission using multiple element arrays by sending multiple copies of the same signal and relying on the multipath richness of the channel to expect independent fades. Diversity and spatial multiplexing are overlapping requirements however, and there has been some research on the diversity-multiplexing trade off. It has been shown that both types of gains can simultaneously be achieved with a trade off between the two [Zheng and Tse, 2003]. These early works laid the basis for MIMO.

\subsection{MIMO channel modelling}

As MIMO performance is dependent on the channels ability to carry independent data streams, it becomes imperative to devise models to predict the propagation environment. The degree of accuracy of these models helps measure the performance of a particular MIMO system and can be used in developing algorithms for MIMO. The initial investigation into MIMO assumed an independent identically distributed Gaussian channel.

\subsubsection{Gesbert's channel model}

Gesbert et al [2002] presented a MIMO channel model for outdoor conditions. This model differentiates between three types of channels: Uncorrelated high rank (iid), uncorrelated low rank (pinhole) and Correlated low rank. Two effects make up the channel transfer function in this model: Scattering effects located close (as compared to the transmitter-receiver separation) to the antennas and the sensor decorrelations. Key hole effect is created when the channel rank collapses due to the scatterers being too close to the antennas and thus only diversity gain is achieved even in the case of 
uncorrelated fading. This model also accounts for the wavelength, separation between antennas in the array, distance between transmitter and receiver, scattering behaviour and the beamwidth of the arrays. The mathematical expression of Gesbert's channel model is given as:

$$
H=1 / \sqrt{S} R_{\theta_{r}, d_{r}}^{\frac{1}{2}} G_{r} R_{\theta_{s}, \frac{1 D_{r}}{s}}^{\frac{1}{2}} G_{t} R_{\theta_{t}, d_{t}}^{\frac{1}{2}}
$$

In this equation

$G_{r}$ and $G_{t}=$ Gain of receiver and transmitter

$S=$ Number of scatterers

$R_{\theta_{r} d_{r}}^{1 / 2}=$ Receive correlation matrix

$R_{\theta_{t}, d_{t}}^{1 / 2}=$ Transmit Correlation matrix

$R_{\theta_{s}, \frac{2 D_{r}}{s}}^{1 / 2}=$ Represents propagation between $\mathrm{n}$ transmit antennas and $\mathrm{s}$ receive scatterers.

\subsubsection{Experimental characterization of MIMO channel}

Statistical channel analysis is useful in generalizing the channel behaviour and determining the usefulness of implementing MIMO. However these lack the detail and fail to account for the individual characteristics of the wireless channel in use which is both time and space variant.

MIMO channel matrix can be measured experimentally by establishing a MIMO link. Channel matrix entries depicting the transfer function between each transmit and receive antenna are found by filtering the received signal and then performing a Fourier transform. This method could be extended to a wideband channel by dividing it into a number of channels which could be assumed to have a coherent bandwidth and thus undergo flat fading. This allows a direct way of getting values for the channel matrix. The channel matrix in this case includes the antenna effects at the 
transmitter and the receiver as well as the channel modes. Though no wireless channel models have been developed so far which could be used for all scattering environments; the experimental deployment of MIMO in different scattering scenarios gives a realistic estimate of the extent to which the theoretical gains of MIMO could be exploited in the real world situations. An example of the practical characterization of a MIMO channel and calculation of capacity is given by Chizhik et al [2003]. In this experiment MIMO system of $16 \times 16$ was used with antennas fixed at a height representing a base station and the others on a mobile van representing mobile receiver. Antennas at the base station were spaced at least $2 \lambda$ apart for adequate decorrelation while mobile antennas could be kept $\lambda / 2$ apart since the scattering is high close to the receiver. Unique continuous wave signals were used at each transmit antenna with a separation of $2 \mathrm{kHz}$ to keep the channel narrowband and avoid any frequency selective effects. The measured channel matrix was inserted into the general MIMO Equation 2.1 and capacity results calculated which were within $80 \%$ of the theoretical Rayleigh channel results. Also the effect of antenna separation on the correlation values was investigated. The base station array showed decorrelation, which did not seem to increase with the separation distance while for the mobile side array, correlation fell off with the increase in the separation between the antennas.

Double directional measurements allow for an accurate and realistic modelling of the radio channel [Bonek and Steinbaner, 2001]. The set-up consists of a channel sounder and transmit and receive antenna arrays. Channel sounders are able to measure various multipath parameters like the amplitude, propagation delay, angle of arrival and Doppler shift etc. The estimated channel transfer function is constructed from these parameters, which is used to obtain the channel capacity. One common method of sounding is to send a modulated pseudorandom sequence down the communication 
link and correlate it with the replica of the sequence at the receiver. MIMO channel sounding is used to characterise the complex channel impulse response of each transmit-receive path in a multi antenna system with regards to time, frequency and space. A suitable multiplexing scheme is required for the receiving antennas to differentiate between transmit antennas which could be based on time, frequency or coding. Sequential or parallel sounders could be used with each having its own advantages and disadvantages. Sequential sounders are based on SISO architecture with the sounding switched between different transmit-receive pairs. This is a low cost and simple to implement method. The switching must however be faster than the coherent time of the channel. Parallel sound ers use parallel transmit-receive arrays. A semi-switched sounding scheme has been introduced by Durham University which is a hybrid of both techniques. It uses parallel receivers and switched transmitter and overcomes the coherence time constraint to some extent [Salous et al., 2005].

Ray tracing has also been used to estimate MIMO channels. It is an analytical method based on the electromagnetic wave theory which gives a deterministic channel model. It gives a site specific result, taking into consideration the electrical characteristics of the antennas and is also useful in validating statistical models. The complexity of a ray tracing model increases when using it for MIMO as computations have to be made for each transmit-receive path. Multi-element capacity calculation have been made using ray tracing models like Wireless Systems Engineering (WiSE) showing the capacity estimations with varying transmit power, received SNR and antenna separations [Chuah et al., 2000]. Methods have been proposed which could reduce the computational burden while modelling reflections, refractions, diffractions and scattering. One of the techniques proposed is image ray tracing [Ng et al., 2007]. 


\subsection{HF antenna arrays}

Antenna arrays have been used in the high frequency band for quite some time. These were deployed as diversity [Van Wambeck and Ross, 1951] and for direction finding applications [Travers et al., 1965] on the receiver side. A very simple high frequency direction finding system (HFDF) was employed by the allies against the German Uboats in the Second World War [Crampton, 1947]. This system used two or more antennas for transmitter site location. Better systems employing spaced arrays were the next step. Arrays were used for source separation, beamforming and to cancel interference by steering the main lobe of the antennas away from the interferer. The signal to noise ratio was improved by making the antenna pattern focus on the direction of arrival and pointing the nulls in the direction of the interferers. Though the concept of using arrays for these applications is different from that of using them for diversity/capacity gains the evolution from one to another is natural.

Radio direction finding systems use two or more receive antennas to identify the location of the transmitted signal. The azimuth angle of the received signal is used to form a triangle with two receivers and a transmitter. Thus having two known nodes of a triangle the third could be easily calculated. Single station location (SSL) systems employ antennas in a single location and use azimuth/elevation to determine the angle of arrival and hence the location of the transmitter. It has been shown that collocated antennas could be employed in direction finding systems [Erhel et al., 1998]. Similarly heterogeneous arrays could be used for the same purpose [Erhel et al., 1997].

The initial experimental implementation of MIMO used spaced antennas [Ling et al., 2002]. These are a good way to avoid SNR fades when multipath signals are delayed by time greater then the reciprocal of bandwidth of the signal. The antennas spacing to provide decorrelation must be placed at least half a wavelength apart in 
order to reduce the effects of mutual coupling [Svantesson and Ranheim, 2001] and introduce sufficient decorrelation [Jakes, 1974] for MIMO to be exploited. Though latest research in VHF/UHF MIMO is also going in the direction of using collocated antennas it is a prerequisite to use space reduction techniques in HF band due to the large wavelengths involved. Multipath propagation in HF is accompanied by multimodal components which are spatially close with elliptical polarization having opposite rotational sense. Polarization diversity could be employed to form biased antennas in favour of one of these modes thus overcoming fading. It has been shown that spaced arrays however large will not be able to resolve these modes and at-least one deep fade is present in the array causing an amplitude deterioration of 20-39 dB [Frazer and Harris, 2006]. Thus collocated antennas not only save space but are also helpful in using the polarization/pattern properties to the advantage of MIMO operation. Collocated and dual polarized antennas have also been shown to give a superior performance when compared to the homogenous spaced arrays in VHF/UHF MIMO [Eiceg et al., 2006].

The use of collocated antennas to improve HF transmissions has been described in the paper by Bisiaux and Bertel [2000]. The experimental setup consisted of 8 collocated receive antennas though only 4 antennas (E-W and N-S vertical square loops, horizontal square loop and an XYZ dipole) were actually used in the experiment. This was a SIMO configuration where one transmitting antenna and multiple receive antennas were used. They used 16-QAM to modulate their $3 \mathrm{kHz}$ signal and the carrier frequency used was $8 \mathrm{MHz}$. The link used was $250 \mathrm{~km}$ long. The antennas response to the incident HF sky wave depends on the vectorial properties of the signal (elevation, azimuth), the polarization (polarization ratio, tilt angle) and is also a function of the shape and orientation of the antenna. For a single HF wave 
following a path there will just be two values of polarization: one for each $\mathrm{O}$ and $\mathrm{X}$ mode. Since the direction of arrival is approximately same for $\mathrm{O}$ and $\mathrm{X}$ modes the signal received by the collocated antennas will just be a function of the polarization and shape of the antenna. Thus antennas could be devised which are able to provide diversity to a HF communication system. The results are shown in Figure 2.1 where all the antennas can be seen to undergo fading, however these fades were independent and a strong signal was present on at least one of the 4 antennas at all time. To check the robustness of this array BER rate was compared with the BER if only the N-S and EW vertical antennas were used. In the latter case BER of $5.3 \times 10^{-2}$ and $1.6 \times 10^{-2}$ was

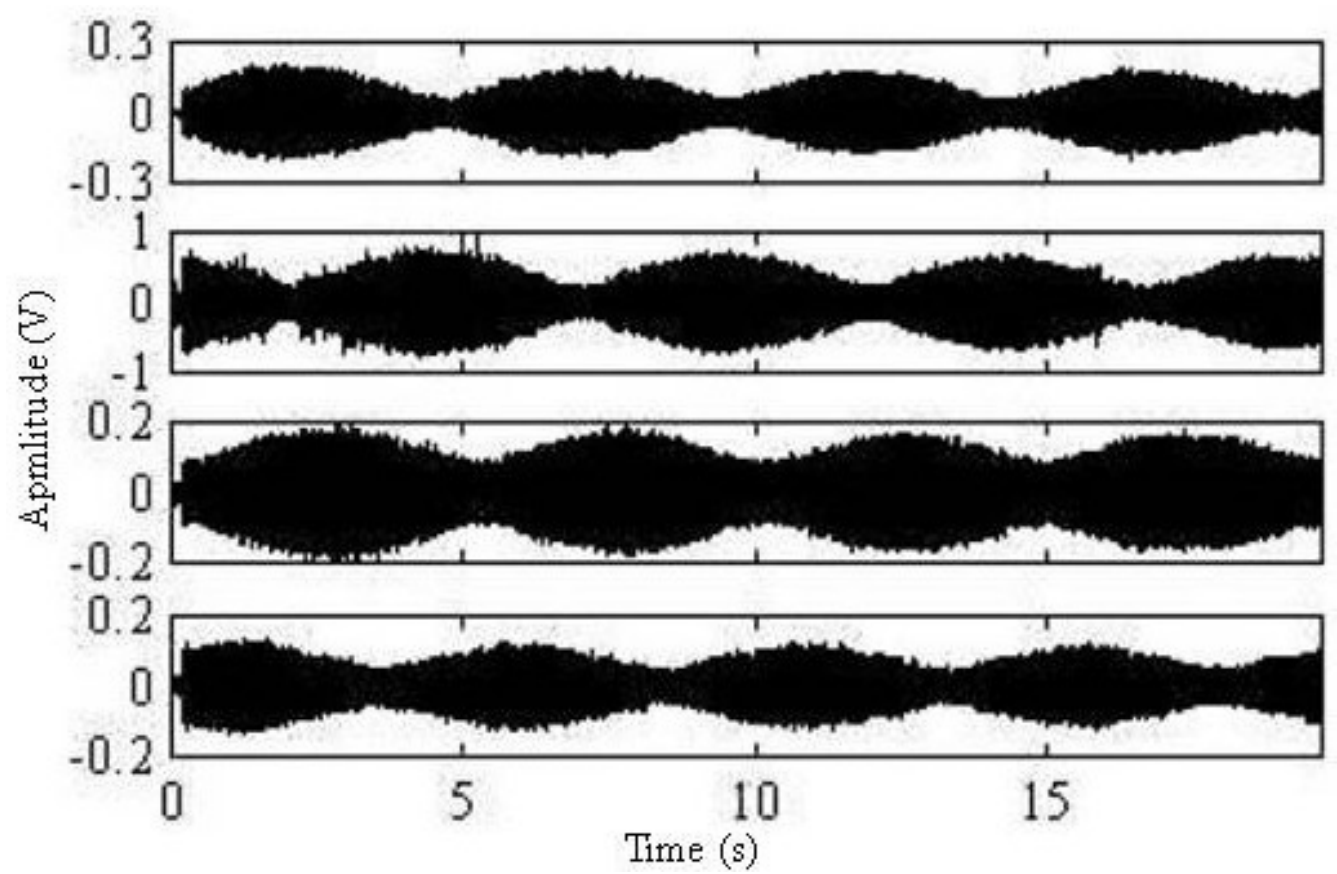

Figure 2.1 Signals received at antennas in the array [Bisiaux and Bertel, 2000]

observed under favourable conditions using simple polarization filter. With an adaptive filter employed the two, three and four element arrays gave a BER of $2 \times 10^{-2}$, $3.7 \times 10^{-3}$ and $2.8 \times 10^{-3}$ respectively. This proves that polarization treatment is not sufficient to overcome fading and some form of pattern diversity has to be employed to make the best use of an array of receive antennas. 
The results described above have been extended to include image transmissions and comparison of the correlations between homogenous and heterogeneous array [Perrine et al., 2006]. The experiment with circularly positioned homogenous and heterogeneous (each antenna rotated by 30 degrees from the adjacent one) sensors revealed that even for two orthogonal signals coming from same direction the heterogeneous arrays offered decorrelation (correlation of 0.87) whereas the homogenous array was correlated as expected. The authors also experimented with image transmission and achieved a data rate of $20 \mathrm{kbps}$ with $6 \mathrm{kHz}$ bandwidth without any error correction applied. A data rate of $30 \mathrm{kbps}$ with $9 \mathrm{kHz}$ bandwidth was achieved using non identical sensors over a $1300 \mathrm{~km}$ path using blind CMA equalizer [Perrine et al., 2006].

The estimation of channel matrix and hence the capacity calculation requires arrays at both the transmitter and the receiver. This is cumbersome in the case of HF where antenna size is large. A technique has been proposed which uses a single transmit antenna and limited number of receive antennas to calculate the capacity of the MIMO channel [Brine et al., 2006]. In this study four-element antenna array is used on the receiver side with a single antenna on the transmit side using frequency modulated continuous wave (FMCW) signals. It forms an oblique ionosonde where the transmitter is sweeping across the range of HF frequencies. Thus ionograms were constructed which were used to obtain results. Three basic types of scenarios are discussed:

1. When antennas in the transmit and receive arrays are closely spaced In this case the transmit antennas will share the propagating modes and this is verified by comparing the ionograms at receive antennas which were found to be similar. The channel matrix in this case is defined as: 


$$
H=R M T
$$

Where $R$ is a $n_{r \times m}$ matrix containing terms representing complex transfer function for all propagating modes impinging at each receive antenna, $M$ is the propagating mode matrix and $T$ is a $n_{m \times t}$ matrix representing complex transfer function between the transmit antennas and the propagating modes present. Rank properties of channel matrix were studied using three different scenarios with the antennas closely spaced such that the antennas pairs share the same propagating modes.

a) Receive antennas are fully correlated while propagating modes and transmit antennas are uncorrelated: In this case channel matrix collapses and has a rank of 1 .

b) Transmit antennas are correlated whereas receive antennas and modes are uncorrelated: Again the channel matrix collapses to give a rank of 1.

c) Propagating modes are correlated while the antennas are uncorrelated: This again gives a rank of 1 . Such an effect where the modes of the channel are correlated no advantage can be gained from MIMO and is termed as the keyhole effect [Chizhik et al., 2002].

2. When transmit antennas are not closely spaced but receive antennas are closely spaced

In this case each transmit antenna would excite different propagating modes and the channel matrix would be made of entries from each transmitter. Thus the channel rank would be higher than when the transmitting antennas are closely spaced represented as:

$$
H=\left[T_{1} T_{2} \ldots T_{n T}\right]
$$

3. When both transmit and receive antennas are not closely spaced

When both the transmitters and receive elements are widely spaced any number of 
MIMO antennas can be used leading to a proportional gains in capacity.

To construct the channel matrix Gesbert's channel model was used as defined in Equation 2.3. Antenna correlations were measured by detecting FFT peaks of the frequency sweep data divided into small blocks for all antennas. Mode correlation measurements on the other hand require only one transmit-receive pair. These were then inserted into the general MIMO capacity equation to determine the capacity. A maximum capacity of $76 \mathrm{bps} / \mathrm{Hz}$ was attained using a channel matrix rank of 8 as compared to $9 \mathrm{bps} / \mathrm{Hz}$ using SISO channel. The revealed modes of propagation depict the rank of the channel matrix which in turn is a guide to using a particular number of antennas for achieving the optimum MIMO capacity. This approach eliminates the need for costly and laborious task of setting up antenna arrays. However it is not a substitute of actually implementing MIMO and finding capacity using the measured channel matrix. This work did not take into account the presence of $\mathrm{O}$ and $\mathrm{X}$ modes in these calculations and no attempt was made to resolve them.

Strangeways [2006] conducted a basic study of the suitability of HF for MIMO which suggests that the time varying small scale irregularities in the ionosphere affect the correlation coefficient between antennas keeping other variables constant and that an increase in variance of these irregularities helps in the decorrelation. In this study SIMO system was simulated using a wide band HF simulator to realize the output of a received signal at the receiver. He used the following formula to find the correlation coefficients:

$$
p(d)=\exp \left(-d^{2} / 2{\sigma_{i}}^{2}\right)
$$

In this equation $p(d)$ is the spatial correlation function, $d$ is the separation between two antennas and $\sigma_{l}^{2}$ is the variance of electron density deviations. According to this formulae correlation decreases with the increase in distance and increases with 
the increase in variance of the electron densities. The correlation coefficients were found for antennas spaced at distances of up to $3.2 \mathrm{~km}$. It was found out that the $\mathrm{F}$ layer decorrelates much more quickly than the E layer possibly because of the higher values of variance in the $\mathrm{F}$ layer. However the low and high $\mathrm{F}$ mode rays do not show an appreciable difference in this respect.

A HF-MIMO system has been proposed in literature which combines the OFDM with space time coding to generate symbols to drive sensors in a multi-antenna system [Shuzheng Xu et al., 2004]. A 2x2 MIMO using Alamouti's space time block coding is used as an example in this case. Maximum ratio combining (MRC) is used at the receiver for the two received signals and the combined output is sent to the maximum likelihood decoder. The simulation results of the scheme with single carrier and OFDM modulation are compared which show that the OFDM in conjunction with MIMO can improve the SNR by as much as $20 \mathrm{~dB}$. Though this research is based on assumptions such as independent fading paths available and perfect channel estimation it gives an idea of what could be achieved in the HF band.

The correlation analysis of two spaced homogenous arrays was done by Gunashekar et al [2007] using data from a previous unrelated study [Warrington, 1986]. This study was based on the results of two different types of antenna arrangements

- Large Forward look array

This array consisted of seven vertical monopole antennas arranged in the form of a $\mathrm{V}$ with one antenna in the vertex and three antennas making each arm of the V. The minimum separation distance between antennas was $177 \mathrm{~m}$ and a maximum spacing of $1526 \mathrm{~m}$. This array is shown in Figure 2.2. 
A shorter path with single-moded propagation was considered for this array. The correlation coefficients were observed to drop down with distance however very large separation between antennas was required for the correlation to reduce significantly as seen in Figure 2.3. For the same path at the same frequency the case of multi-moded communication the correlation coefficients were observed to drop down much more rapidly with distance. In addition orientation dependent effects were also observed.

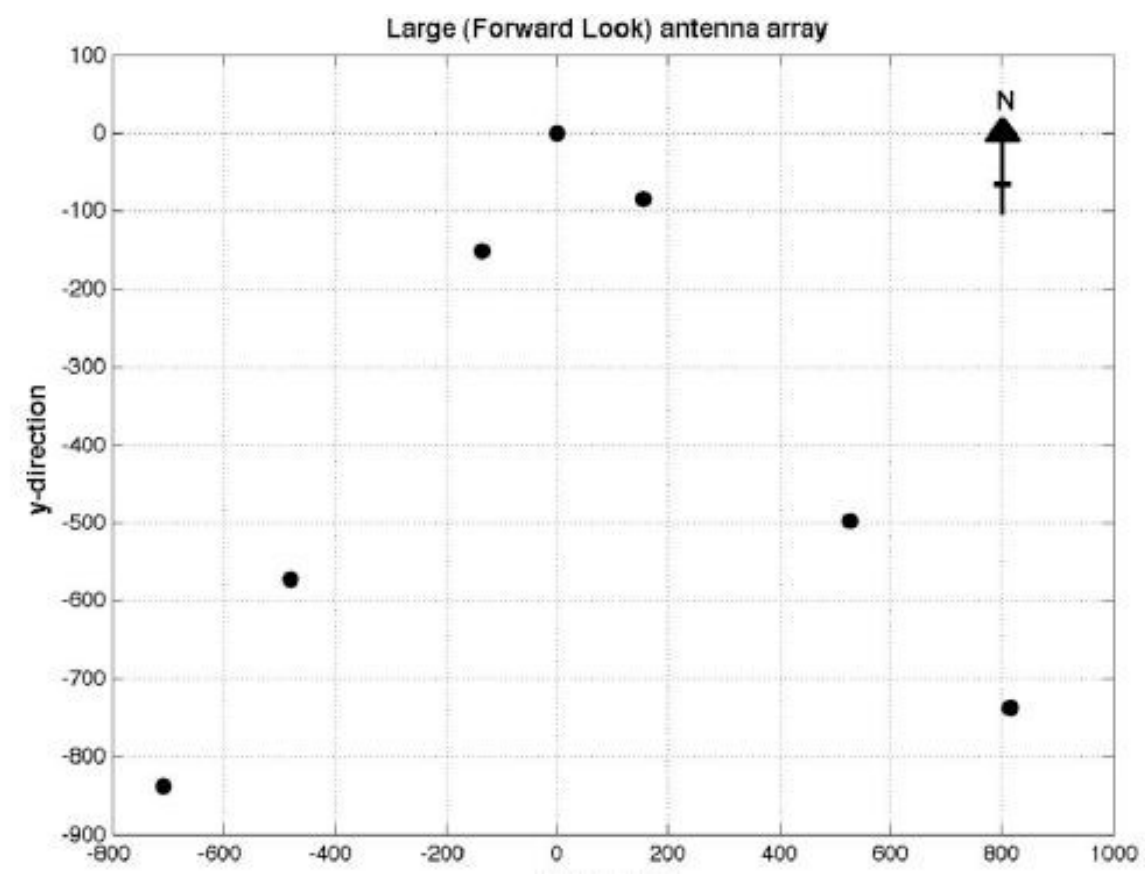

Figure 2.2 The relative positions of the vertical monopoles employed in the large receiving antenna array (Forward look) [Gunashekar et al, 2007].

- Small Verbena array

This array was made up of 24 vertical monopoles arranged in a Y configuration. It was the smaller arrays with the largest dimension of 294 metres and 7 of the antennas arranged in a V configuration (Figure 2.5).

In this case a longer path was used with multi-moded communication and the correlation coefficients were observed to fall of with distance much more quickly than the large array (Figure 2.6). The analysis of the data showed that the decorrelation 
between the homogenous antenna pairs was a function of the separation distance between antennas and their orientation.

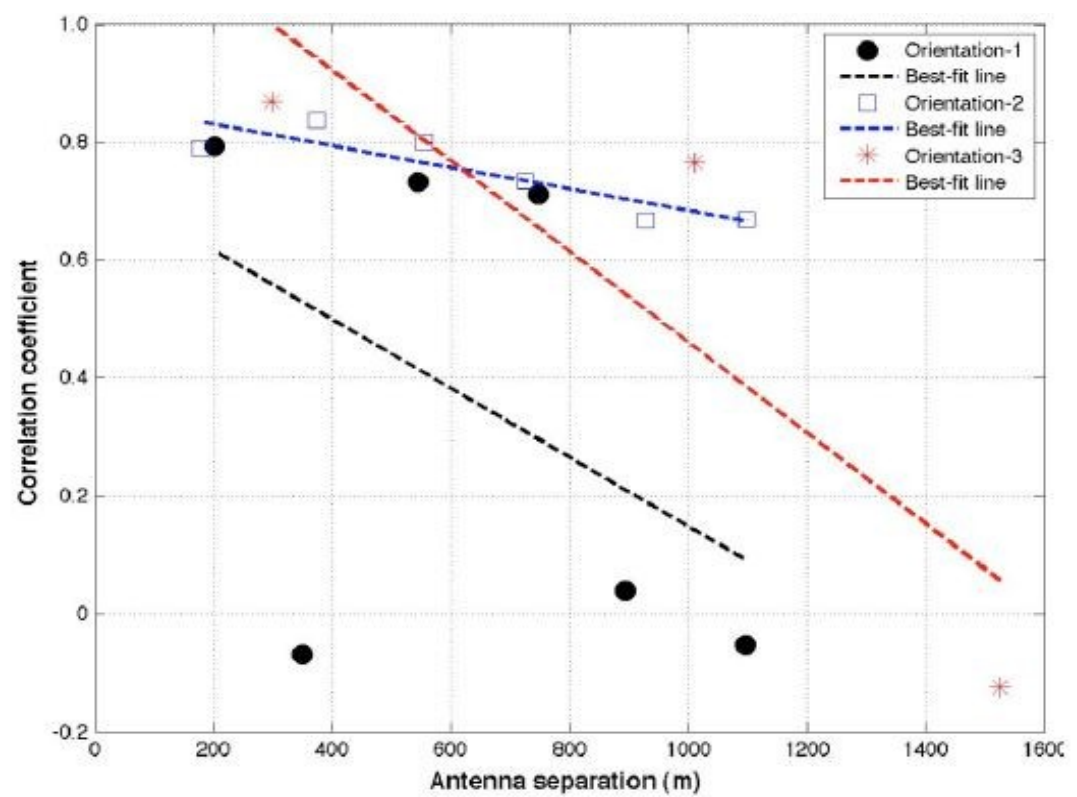

Figure 2.3 Correlation coefficients (single-moded) computed for various anten na pair s pacing's for the large 7-element array with data divided into three parts depending on the orientation of the particular antenna arm (orientation 1: left ar m, orientation 2: right arm, orientation 3: eastwest pairs) [Gun ashekar et al, 2007].

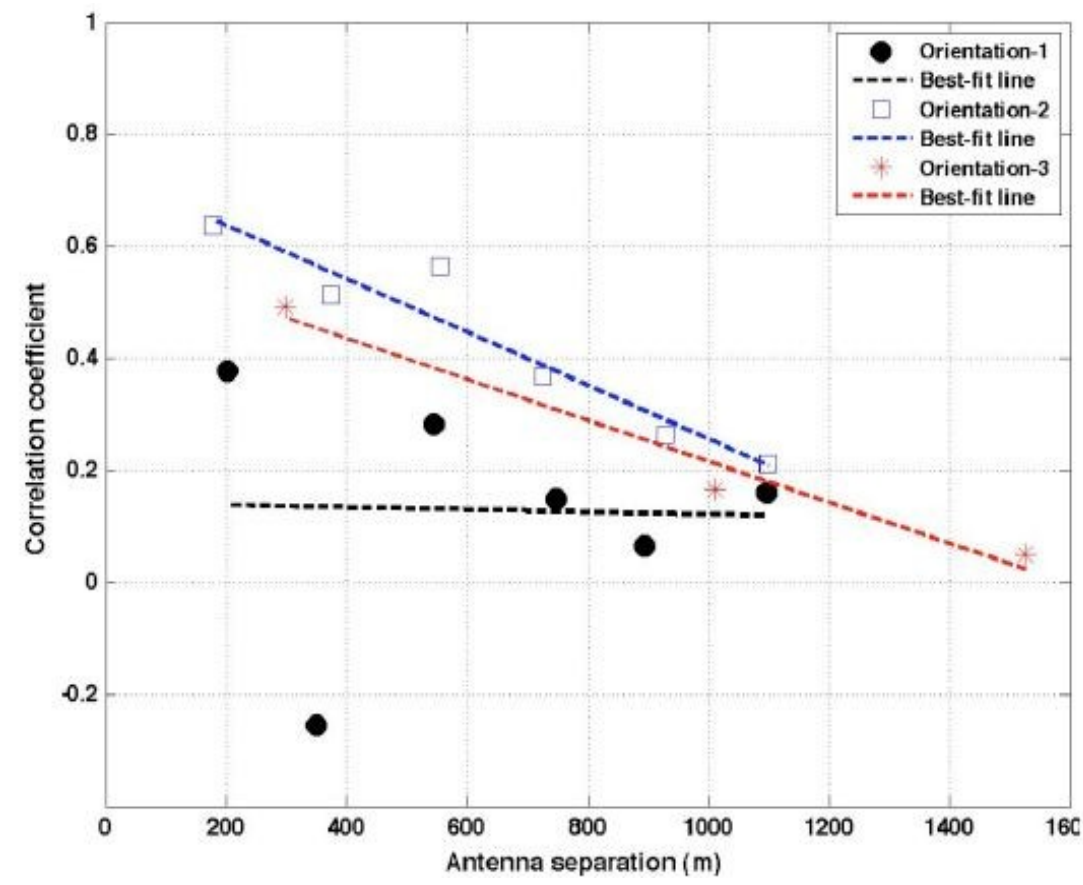

Figure 2.4 Correlation coefficients (multi-moded) computed for various antenna pair s pacing's for the large 7-element array with data divided into three parts depending on the orientation of the particular antenna arm (orientation 1: left arm, orientation 2: right arm, orientation 3: east-west pairs) [Gun as hekar et al, 2007] 


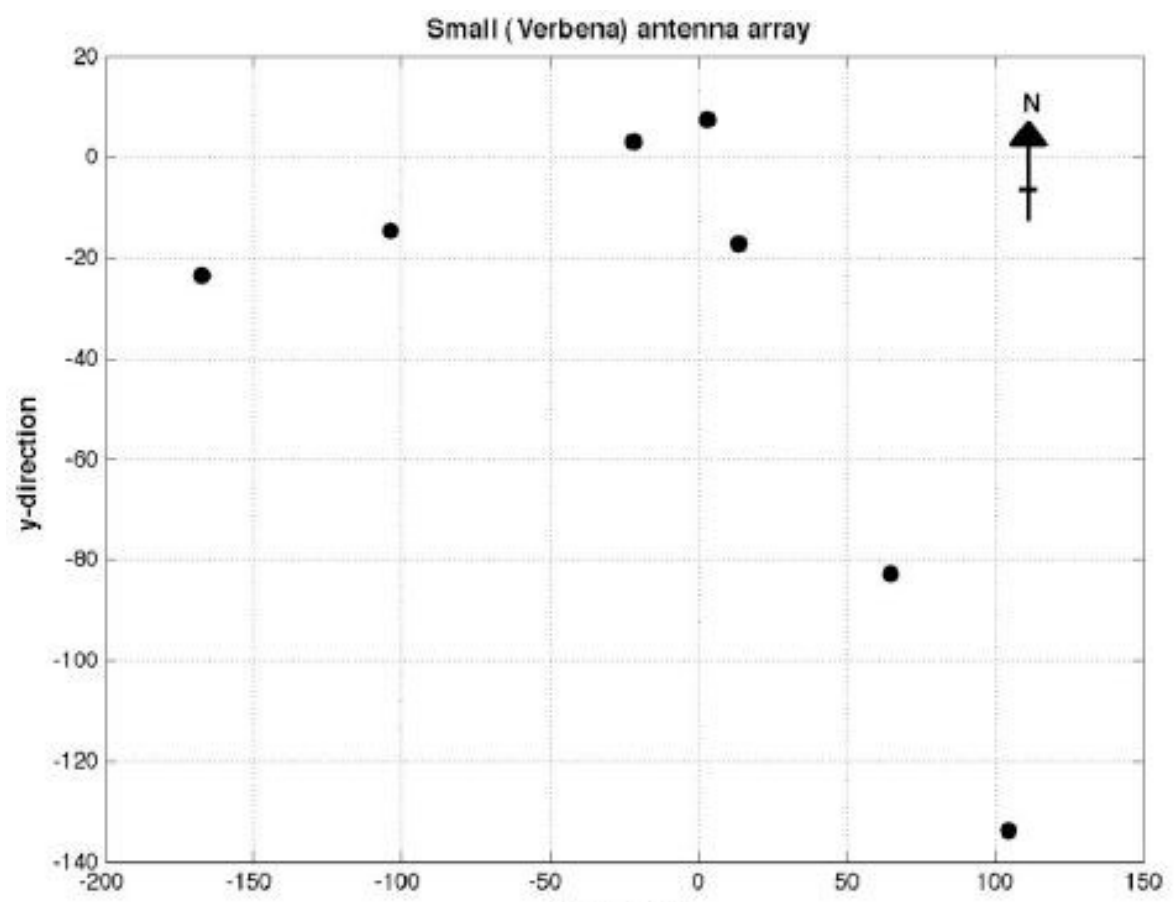

Figure 2.5 The relative positions of the vertical monopoles empl oyed in the s mall receiving antenna array (Verbena)

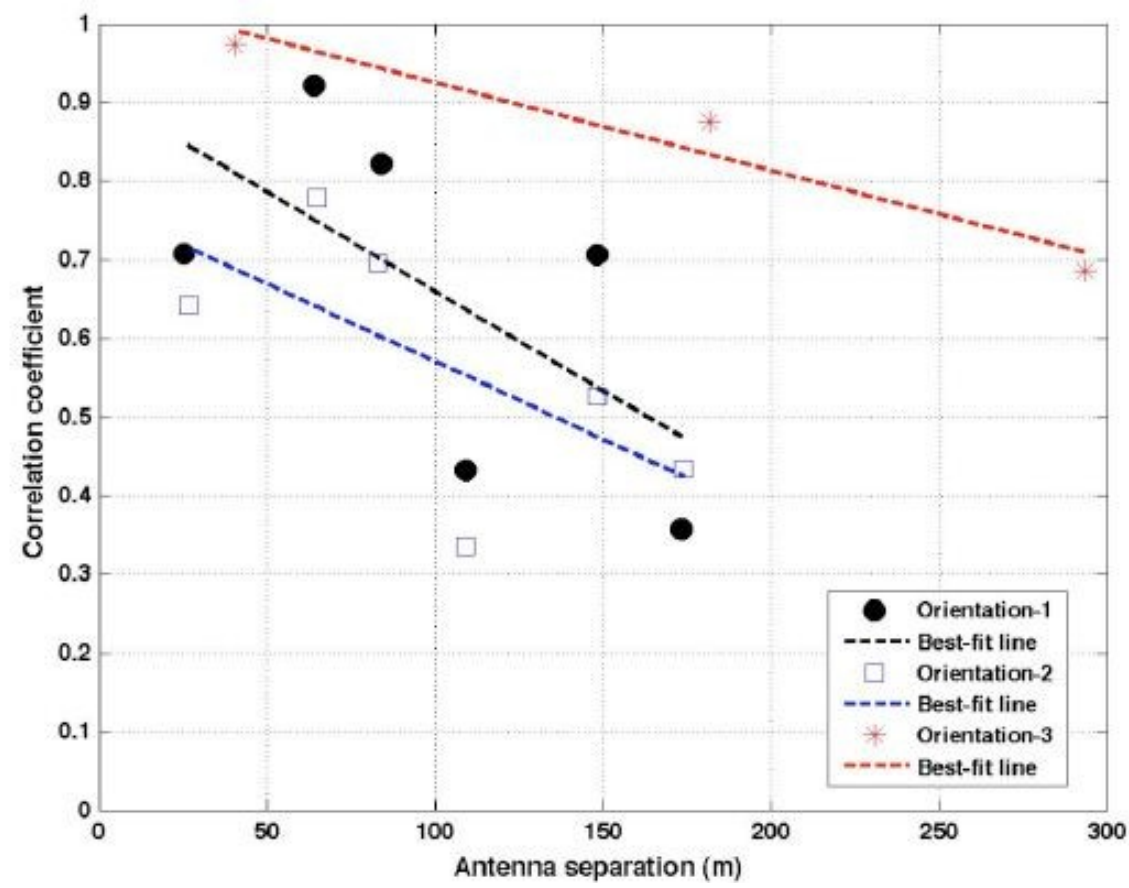

Figure 2.6 Correlation coefficients computed for various antenna s pacings for the small 7-element antenna array with data divided into three parts depending on the orientation of the particular antenna arm (orientation 1: left arm, orientation 2: right arm, orientation 3: east-west pairs) 
The above section gives details of the research carried out using multiple antennas on the HF band. Both spaced and collocated antenna arrays have been utilized in various HF applications from direction finding to diversity and improvement of the bit rate. Antenna decorrelations in spaced and collocated arrays have also been studied. These research initiatives provide a basis for the application of MIMO techniques in the HF band which seems to be the logical next step. However a detailed and comprehensive treatment of the subject is lacking and MIMO in its true sense has not been experimented with as of now. Most of the experimental studies have used single input multiple output (SIMO) antennas and others have tried to study the correlation effects without actual implementation of a multiple input multiple output system in the HF band. This thesis is about the experiments conducted with multiple-transmit and multiple-receive antennas in the HF band using antennas arrays of various types and analysing the received signals for decorrelation and capacity. Also a major problem of using MIMO at HF frequencies i.e. the size of antennas and the array is tackled by introducing large collocated antennas and then further reducing the footprint of the array by utilizing electrically small antennas. 


\section{Experimental Setup}

To study the utilization of MIMO in the HF band dedicated transmitter and receiver sites were used The majority of the experiments were conducted over the $255 \mathrm{~km}$ path between science park, Durham $(54.42 \mathrm{~N}, 1.45 \mathrm{~W})$ and Bruntingthorpe, Leicester $(52.49 \mathrm{~N}, 1.13 \mathrm{~W})$ as shown in Figure 3.1.

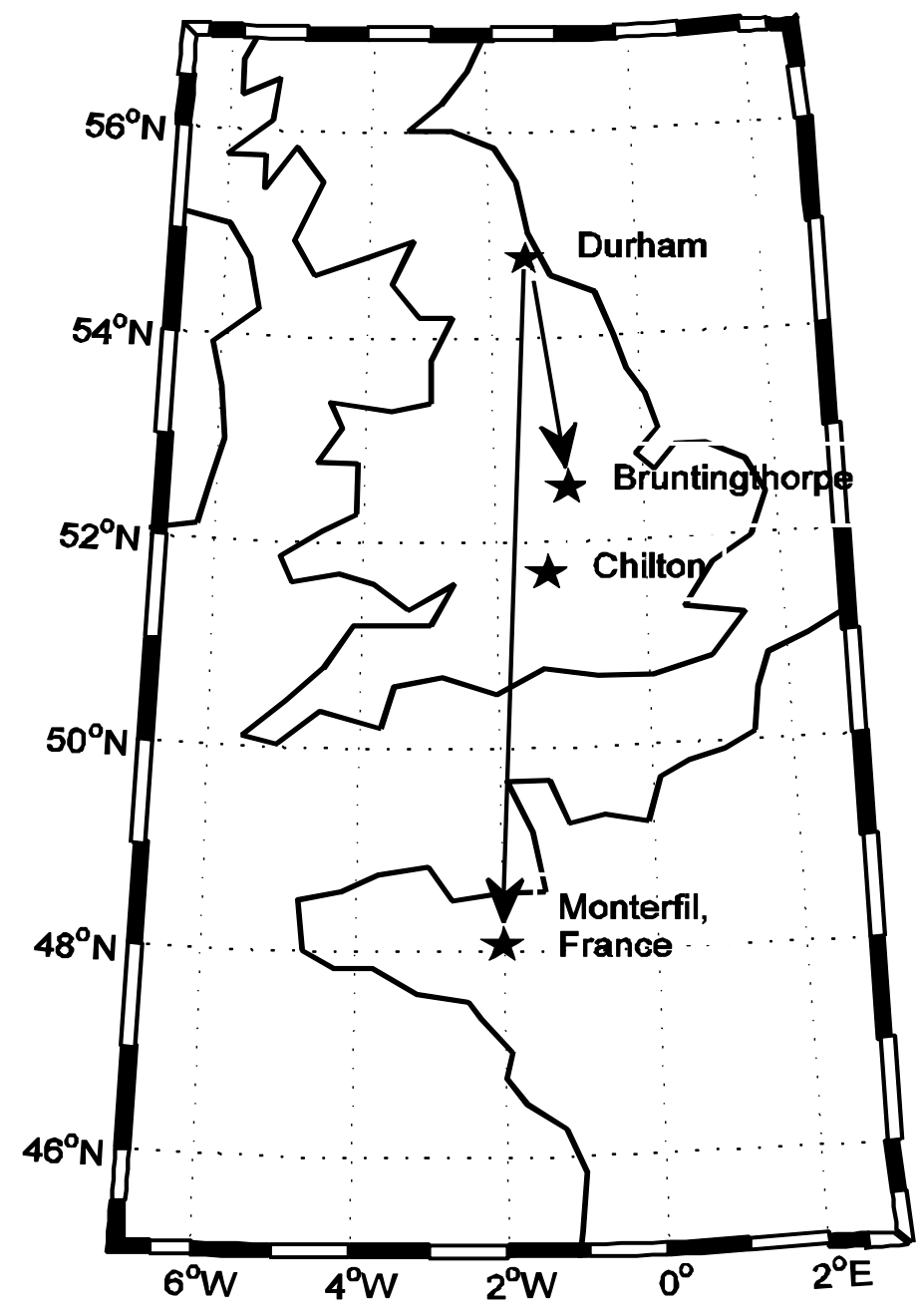

Figure 3.1 Durham - Bruntingthor pe path and Durham-Monterfil path

The transmitter was placed at the site in Durham while Leicester was used as a receiving site exclusively. Continuous wave $(\mathrm{CW})$ transmissions were used in these experiments with a selection of frequencies depending on the propagation condition. The frequencies available for use (2.380 MHz, 3.950 MHz, 4.4455 MHz, 4.636 MHz, 


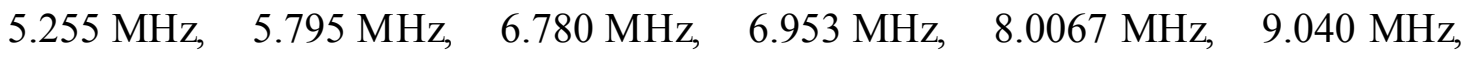
9.428 MHz, $9.940 \mathrm{MHz}, \quad 10.390 \mathrm{MHz}, \quad 11.117 \mathrm{MHz}, \quad 11.175 \mathrm{MHz}, \quad 14.360 \mathrm{MHz}$ 14.363 MHz, 17.450 MHz, 18.380 MHz, $19.950 \mathrm{MHz}$ and 21.880 MHz) were authorized under licence number 262651 to University of Leicester.

For the purpose of selecting the frequency of operation for the particular day and time, ionograms from the Chilton ionosonde were used. These were obtained from the UKSSDC website which is continuously updated and latest ionograms with a lag of approximately 1 hour are available. Transmissions curves corresponding to the Durham-Leicester path plotted over the ionograms were used to select the frequency to be used for transmission from the set of licensed frequencies available.

\subsection{Receive antennas}

The receiving site at Bruntingthorpe, Leicester provided enough area to allow spaced $\mathrm{HF}$ antennas to be placed apart to work as arrays. A variety of receive antenna structures were used starting from the general purpose antennas available in the market and then moving on to building small collocated antennas for the specific purpose of the HF-MIMO investigations. The receive antennas used in the experiments are described below:

\subsubsection{Vertical Monopoles}

Vertical monopole antennas allow omni directional reception and are easy to setup. They occupy very small space and hence are useful for spaced array applications. Initially five spaced vertical monopoles were used in an array. The height of each of these antennas was about $5 \mathrm{~m}$ above the ground and was held in place using guy ropes. These antennas were placed to form an L shaped array as shown in Figure 3.2 with one arm pointing towards north (transmitter) and the other pointing towards east. Thus 
minimum separating distance of $13.3 \mathrm{~m}$ was maintained which is approximately equal to half the wavelength at $10 \mathrm{MHz}$. This set-up allowed for the examination of spatial as well as orientation dependent effects.

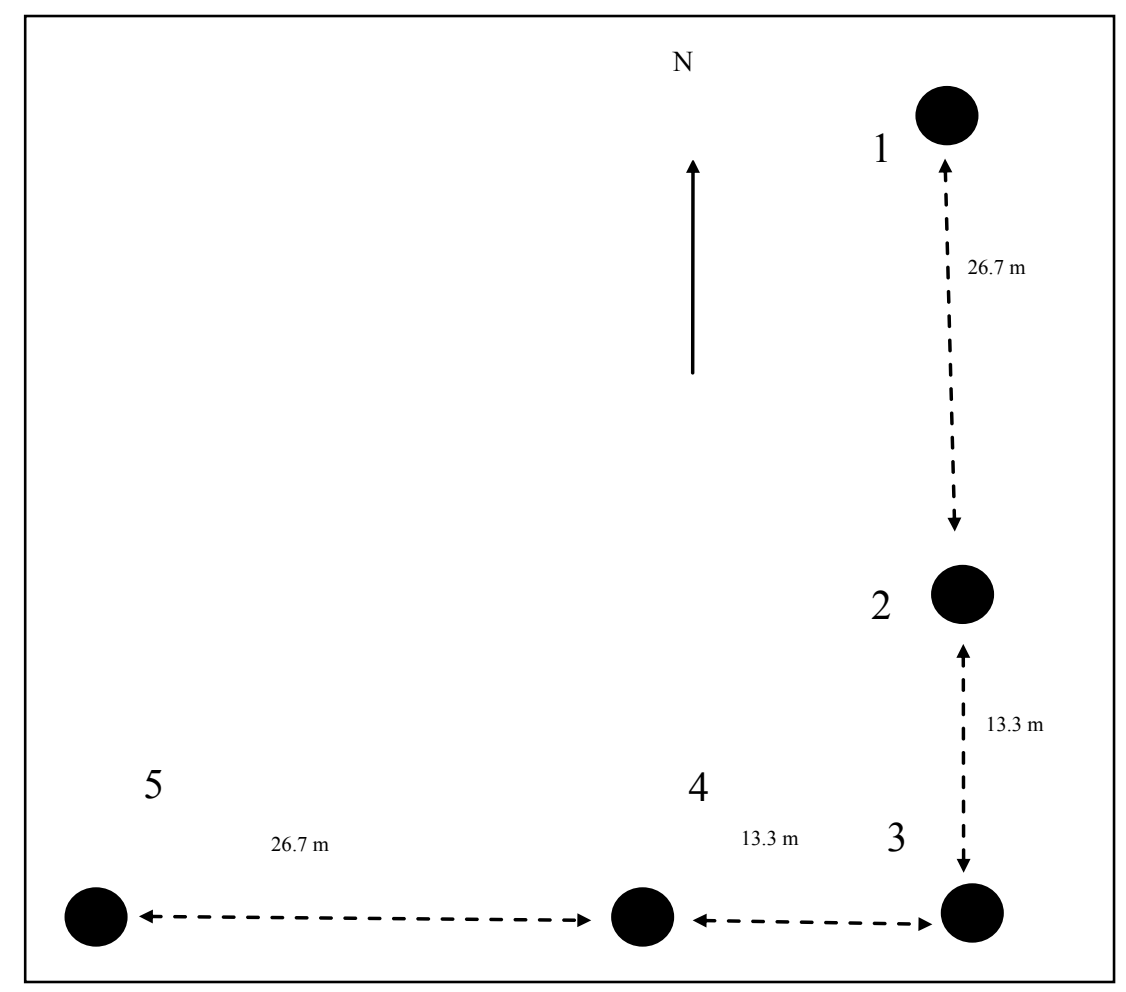

Figure 3.2 Spaced vertical monopole antennas

Monopole antennas were also used in an orthogonal configuration in the form of a collocated array. Active antenna elements were used in this array which allow for the reduction in size by increasing the electrical length of the antenna. Initially the array was placed on ground as shown in Figure 3.3 but later was raised to $3.05 \mathrm{~m}$ above the ground using a mast as depicted in Figure 3.4.

Another array (Figure 3.5) had two horizontal crossed dipole antennas along with a vertical monopole. Active antenna elements were used in this array as well. The dipoles were of a length of $70 \mathrm{~cm}$ and the monopole was $1 \mathrm{~m}$ high and placed in the middle. Balanced amplifiers along with a transformer were used in these antennas. 


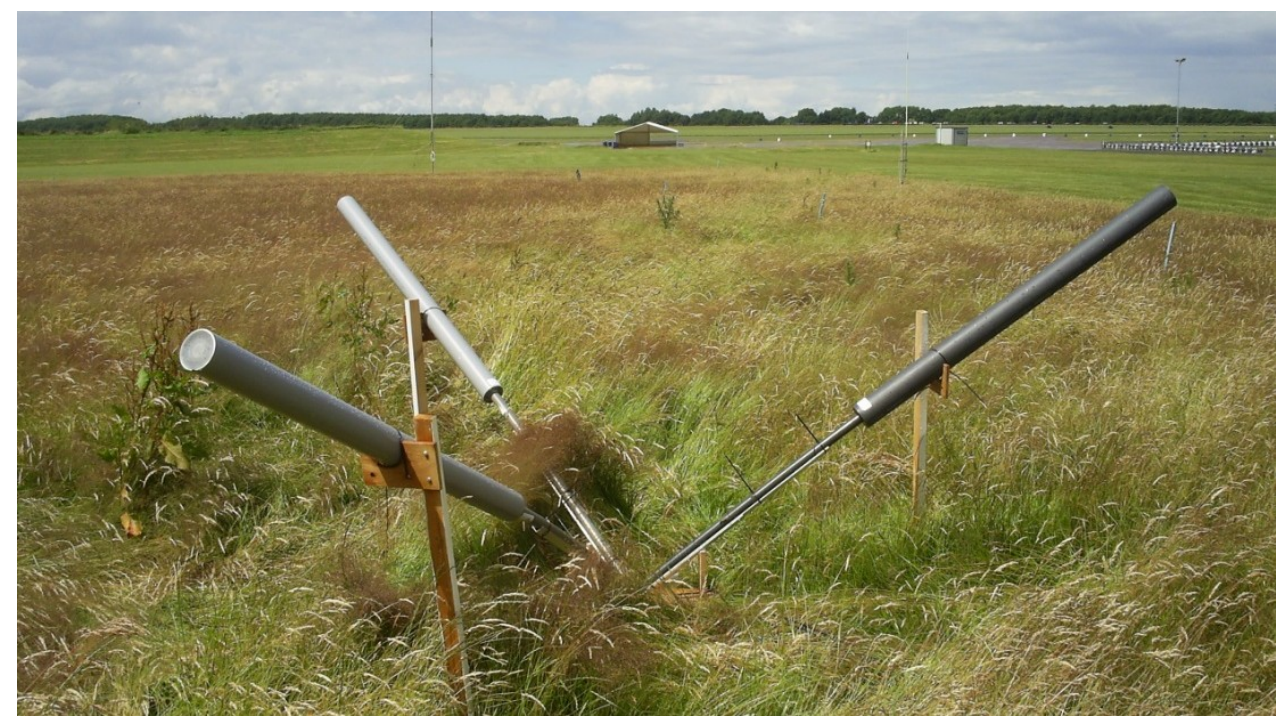

Figure 3.3 Three or thogonal active monopoles at ground level

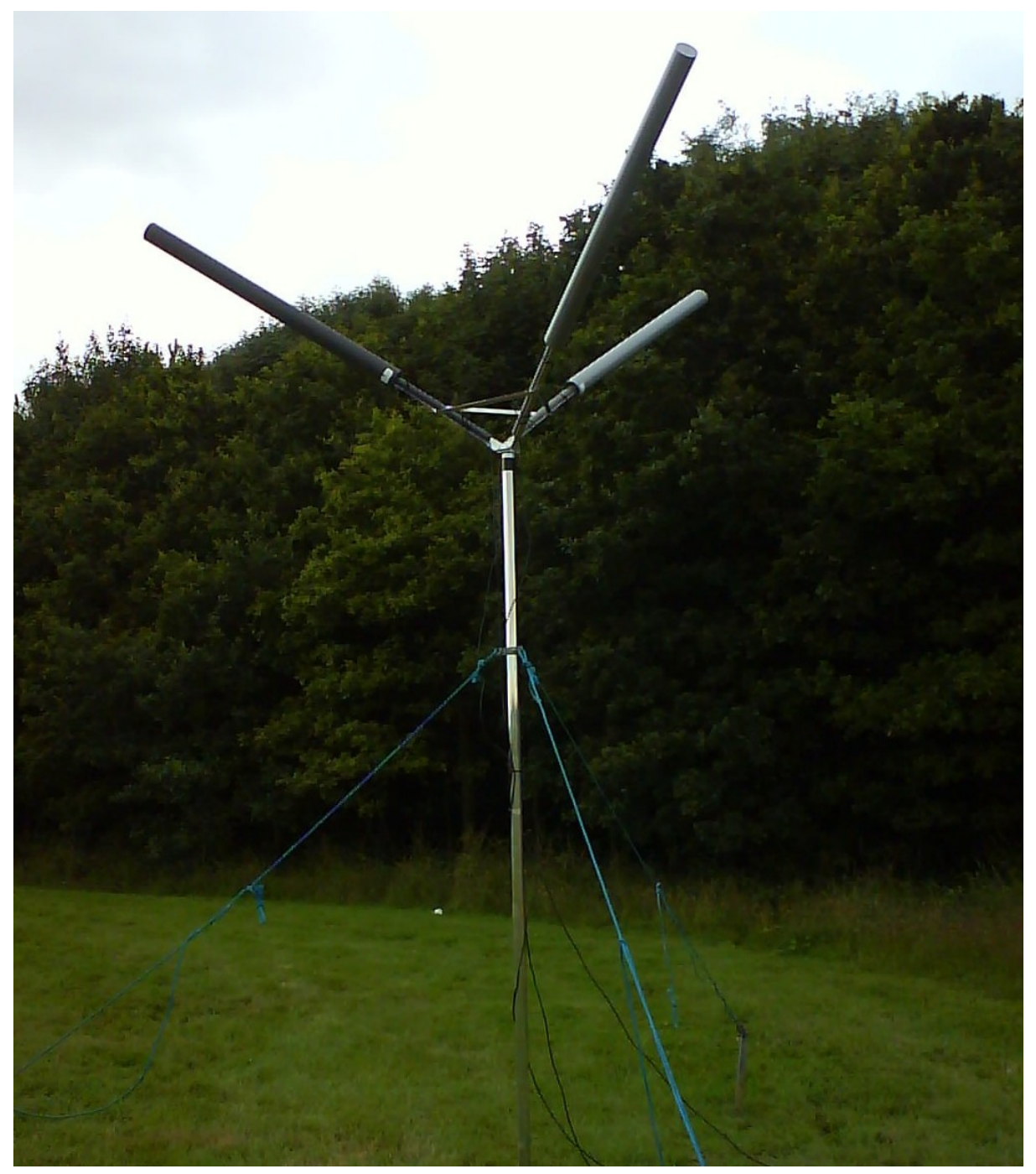

Figure 3.4 Three active monopoles raised above ground level 


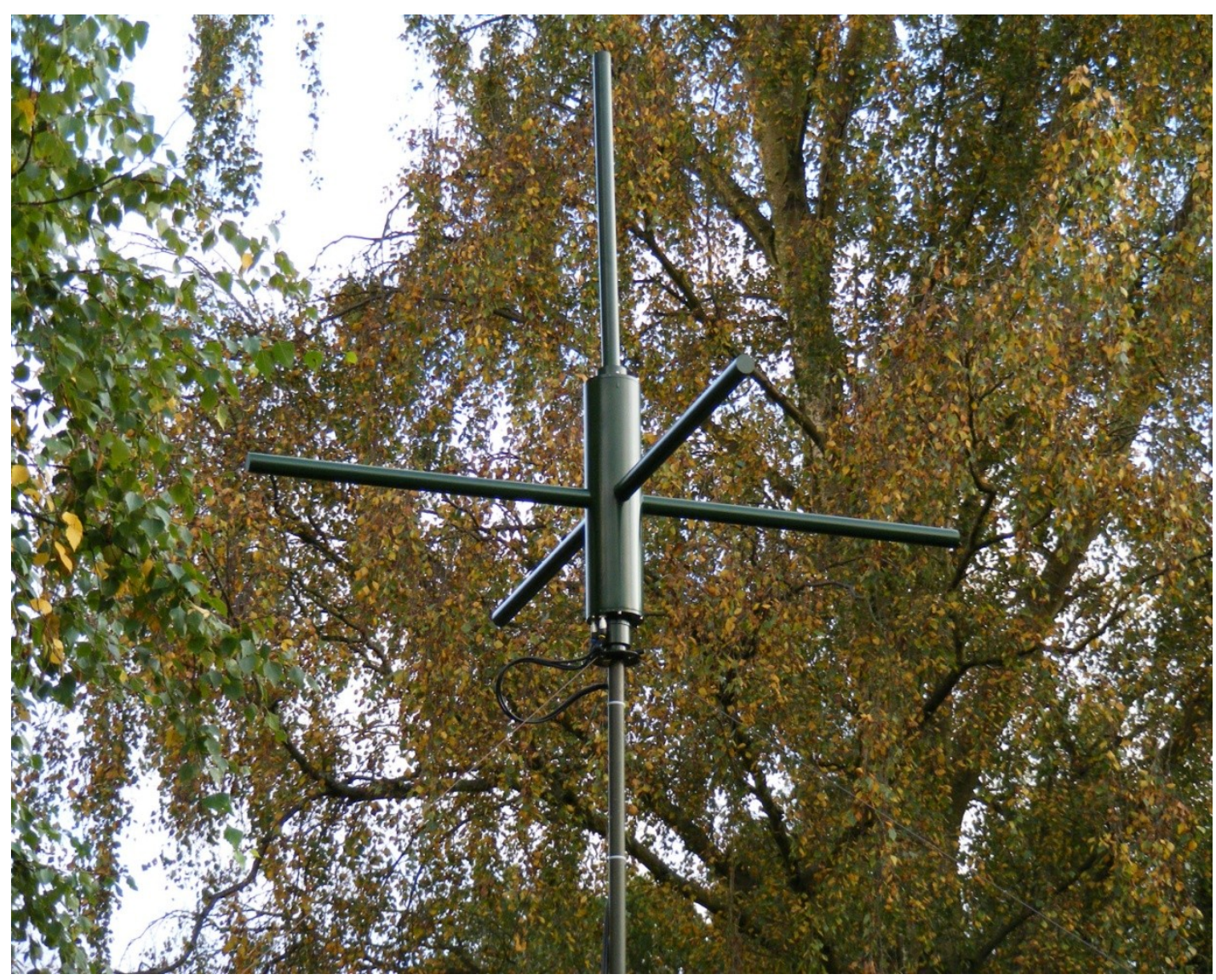

Figure 3.5 Crossed dipoles wi th a central monopole antenna

\subsubsection{Crossed wire inverted $V$ antennas}

Inverted Vs are simple wire antennas which provide a good reception over the entire HF band. The antennas used in our experiments were made from No. 14 AWG of stranded copper wire with $50 \Omega$ feed impedance. It is claimed to give a VSWR of 2:1 for the whole range of frequencies from 1.8 to $30 \mathrm{MHz}$. We had two of these oriented north-south and east-west in a collocated arrangement, sharing the same mast. The base of each antenna was $28 \mathrm{~m}$ long. The height of the antennas above the ground was $7.6 \mathrm{~m}$ where these were supported by a mast. Each antenna had a balun at the source end to cater for the unbalanced feed-line. The single inverted $\mathrm{V}$ antenna is shown in Figure 3.6. 


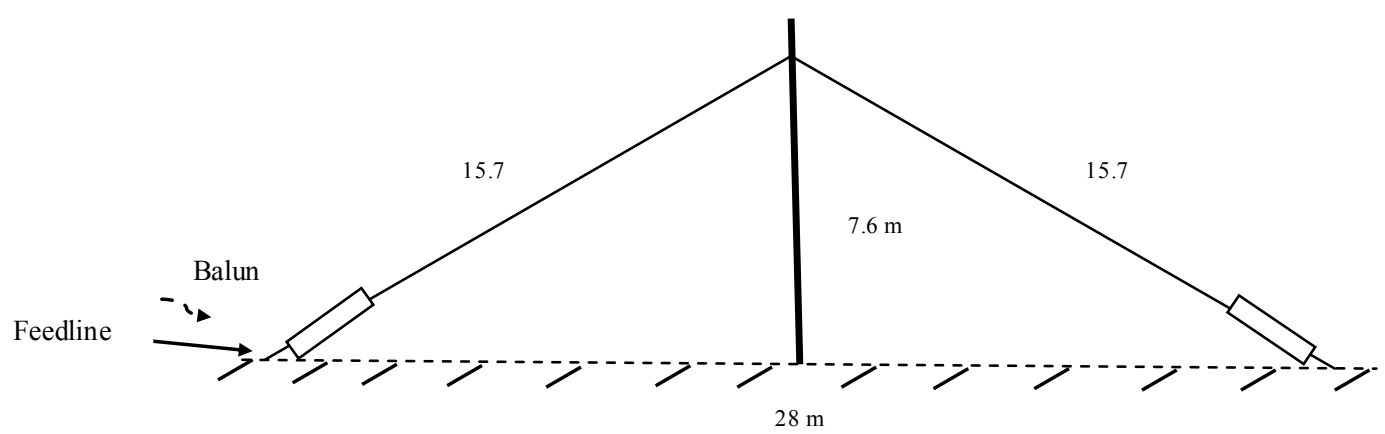

Figure 3.6 Inver ted $V$ wire antenna

\subsubsection{Collocated loop Antennas}

Electrically small loop antennas, also called as magnetic loops have poor efficiency but make good receive antennas. Two kinds of loop arrays were constructed at Durham University for HF-MIMO experiments and are described below:

XYZ loop array (Figure 3.7) had 3 loops oriented orthogonal to each other and mounted on a $3 \mathrm{~m}$ high mast. These consisted of two vertical loops in the north-south and east-west direction and a third horizontal loop at the base. These loops were built with coaxial cables and placed in housing made of PVC plumbing material for mechanical support. The inner conductor of the coax forms the loop while the outer braid is disconnected in the middle to form an open circuited covering for electrostatic shielding. The area of the loop array is $1 \mathrm{~m}^{2}$. Balance amplifiers were used to reduce return losses.

Giselle antenna consists of three collocated loops arranged such that equilateral triangles are formed at the base and the top. It was originally built for direction finding (DF) proposes based on the premise that elliptically polarized wavefields impinging on the different elements would be vertical to the directions of arrival of the signal and can be found out using three orthogonal polarized antennas that are collocated [Massie et al, 2004]. The antenna has a geometry which allows the same sensor shape for each antenna as seen from the ground. The antenna elements were 
thought to pick up the complementary components of the elliptically polarized wave. The phase of the complex signal of a number of samples could then be used to construct a circular plot which depicts the direction of arrival of the required signal [Massie et al., 2004].

The design of this antenna was based on the original model of three collocated square loop antennas [Massie et al., 2004] [Martinsen, 2009]. Each antenna had two turns of loops (each $1 \mathrm{~m}^{2}$ ) giving an impedance of 377 ohms [Feeney et al., 2009]. Matching with the $50 \mathrm{ohm}$ coaxial cable was done using an autotransformer. This design collocates antennas by slanting them at an angle of 60 degrees with respect to ground and thus creating inverted equilateral triangles at the bottom and top. This structure forms symmetry with the ground and has same distributed parameters with

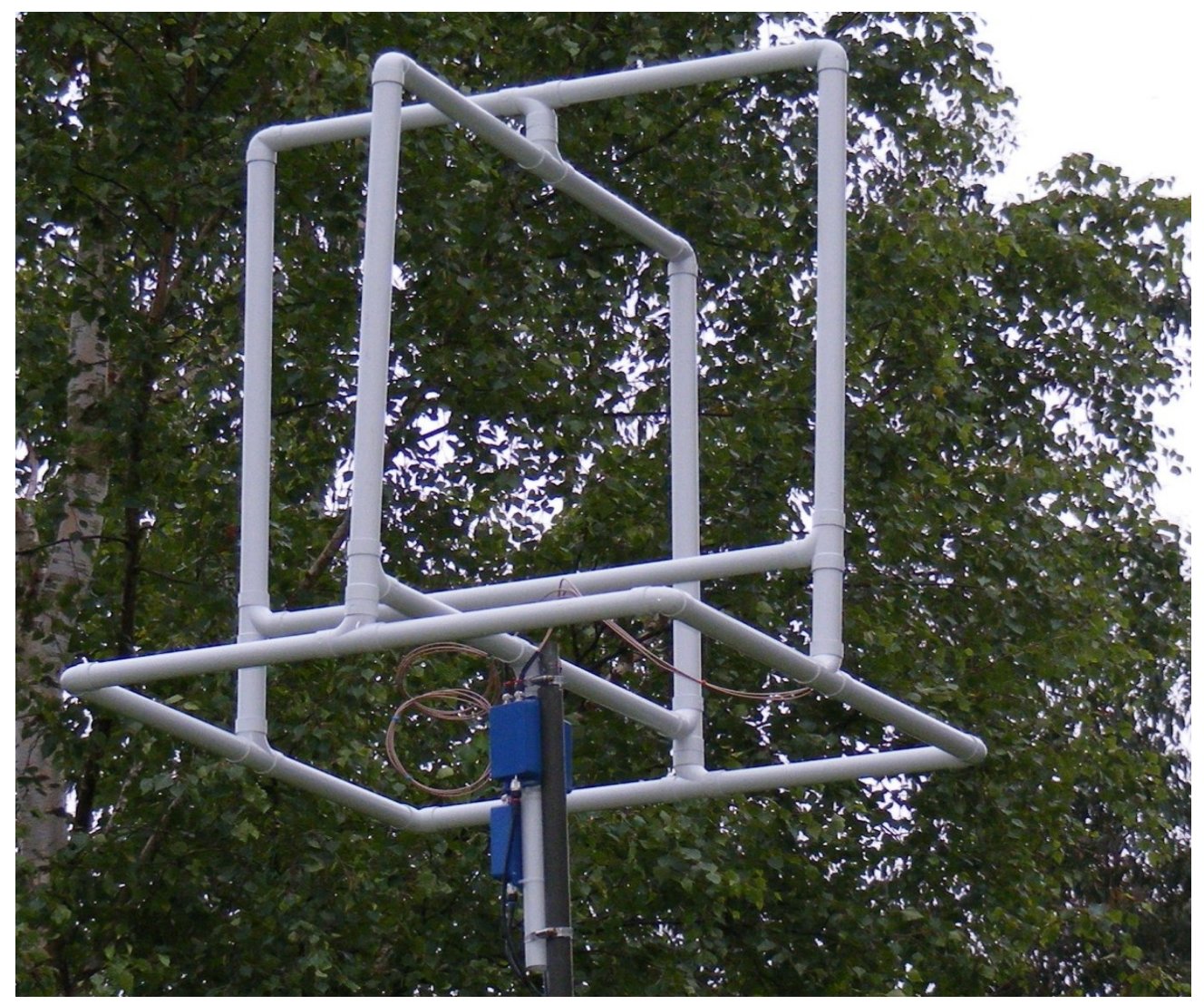

Figure 3.7 XYZ colloc ated loops 


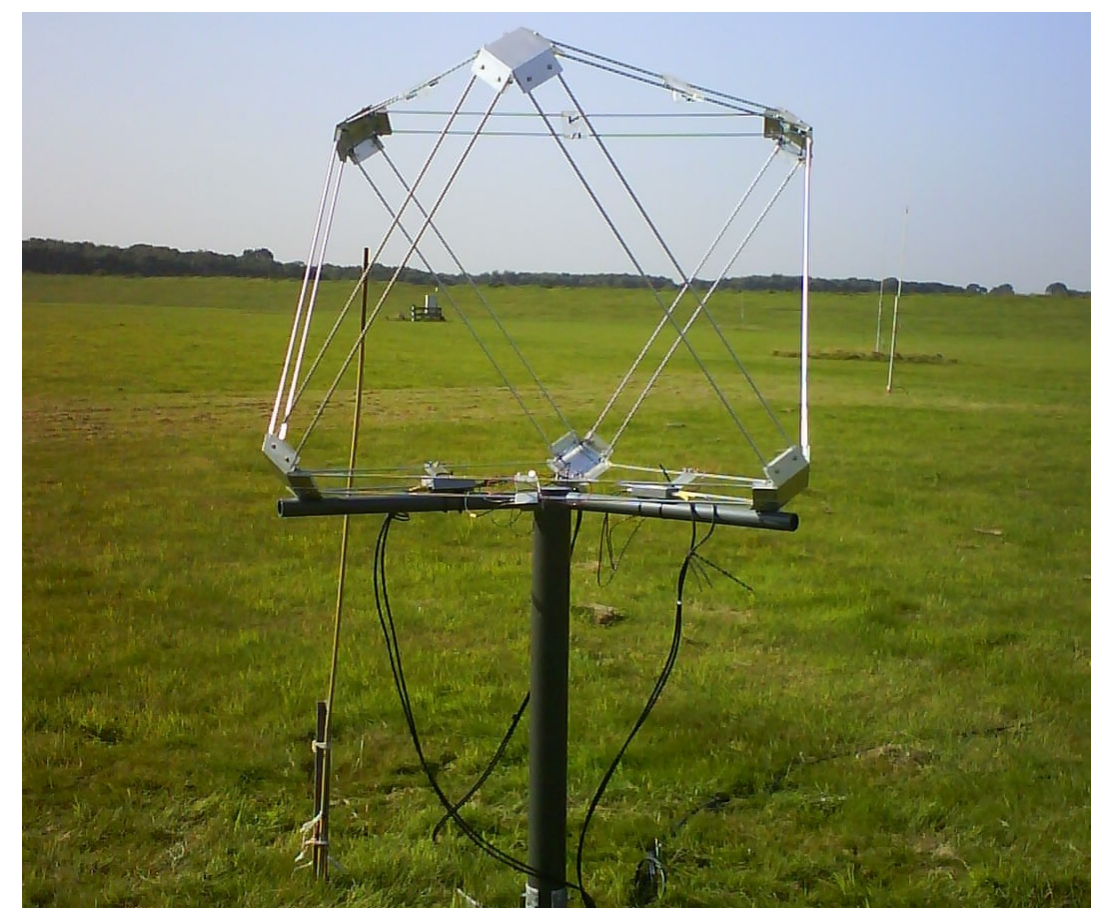

Figure 3.8 Giselle antenna made on the model of the design by DSTO, Australia

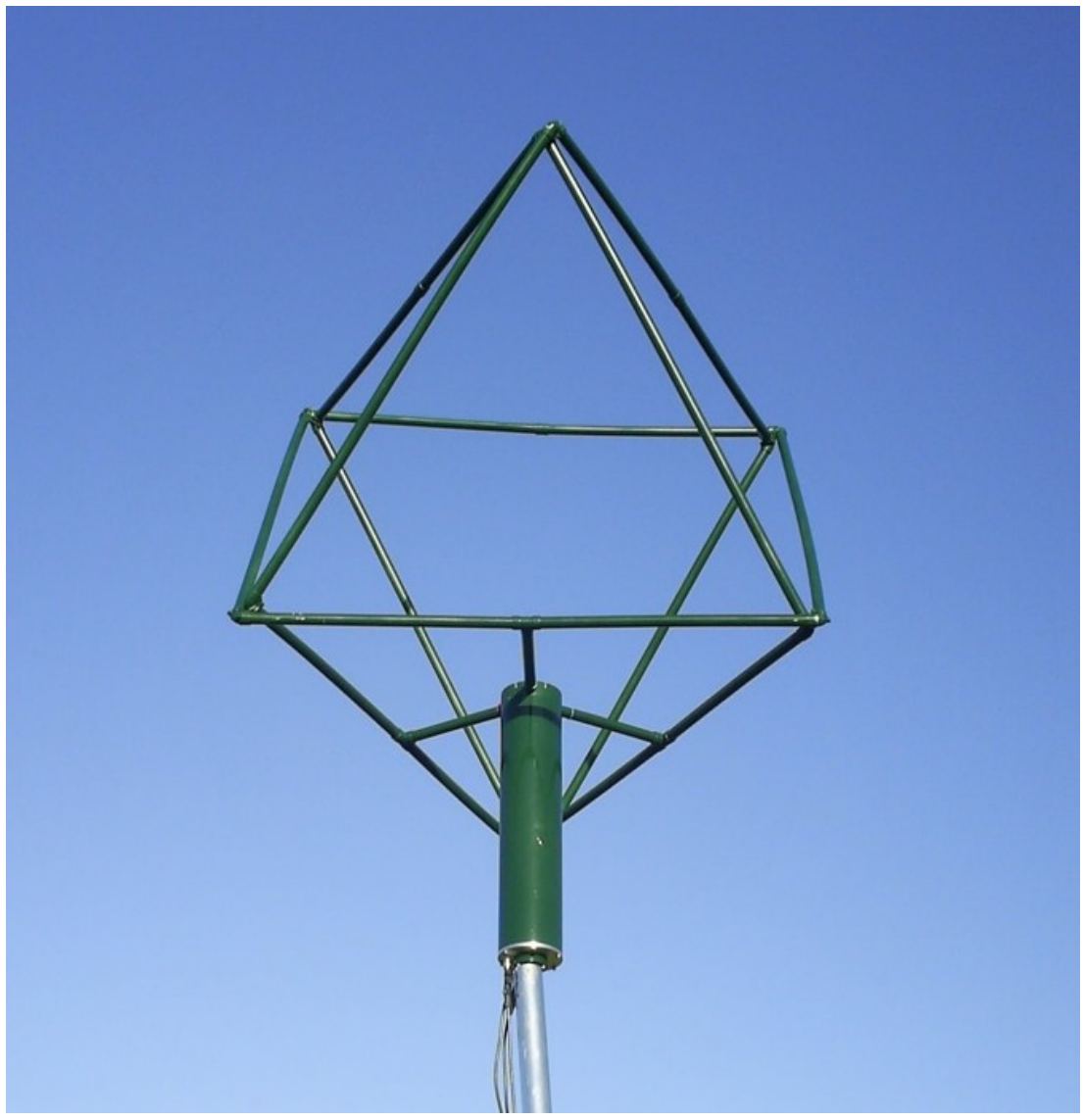

Figure 3.9 Modified design of Giselle antenna constructed at Durham Uni versity 
respect to ground as obtained by each antenna in the array. The antenna constructed according to the original design is shown in Figure 3.8. The original design was replicated without success as signal reception was very poor which was attributed to the $377 \mathrm{ohm}$ impedance matching [Feeney et al., 2009]. Thus a modified design was used, built at Durham University which utilized magnetically coupled loops with balanced amplifiers and matching networks. This new array is shown in Figure 3.9.

\subsection{Transmitting antennas}

The transmitting antennas had to be designed to accommodate the change in the frequency of operation due to the changes in ionospheric conditions. Also since the frequencies have to be selected and changed with short notice the antennas must be able to be tuned in automatically or remotely for the range of frequencies used.

For the initial experiments with HF-MIMO physically large antennas were used. These consisted of matched $7 \mathrm{~m}$ whips as shown in Figure 3.10 and the inverted $\mathrm{V}$ antennas. The inverted $\mathrm{V}$ antennas used had a base length of $30 \mathrm{~m}$ and height of $8 \mathrm{~m}$ and were resistively terminated. In addition loops were also used with matching networks and amplifier circuits as shown in Figure 3.11.

Later the development of compact antennas was initiated and a collocated octagonal transmitting antenna was built at Durham. It consisted of two vertical loops forming a $1.5 \times 1.5 \mathrm{~m}$ octagon. The coaxial cables forming the loops were housed in octagonal shaped hollow aluminium housing. The loops were tuned using a 5-500 pF capacitor and a transformer was used to provide balanced input to the antenna. The antennas exhibit low VSWR values below $15 \mathrm{MHz}$ and have an efficiency ranging from $88 \%$ to $98 \%$ in this region. The $10: 1$ scaled model was used in an anechoic 
chamber to determine the radiation patterns of these antennas [Feeney et al., 2009]. It is shown in Figure 3.12.

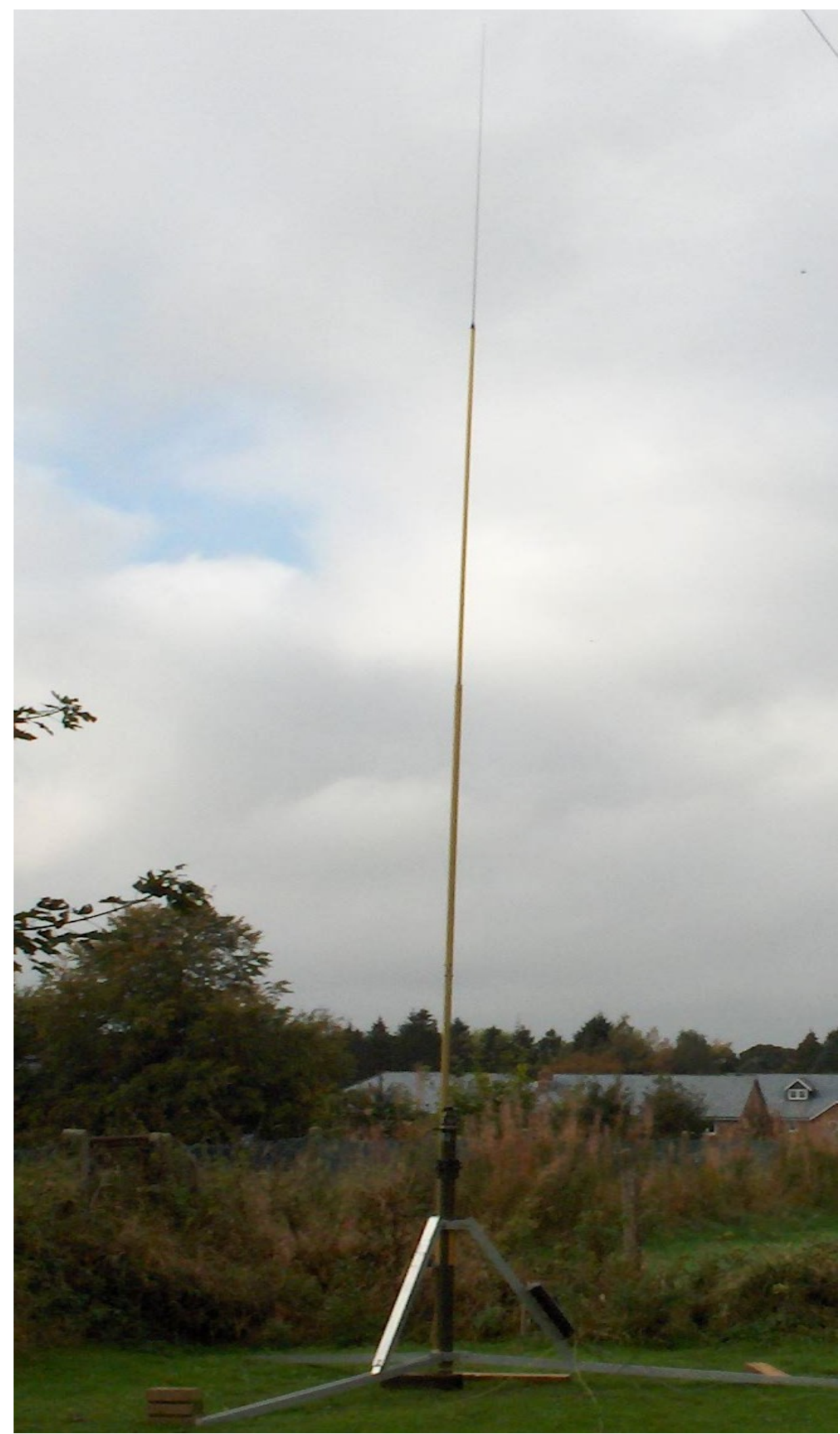

Figure 3.10 Transmitting vertical whip antenna 


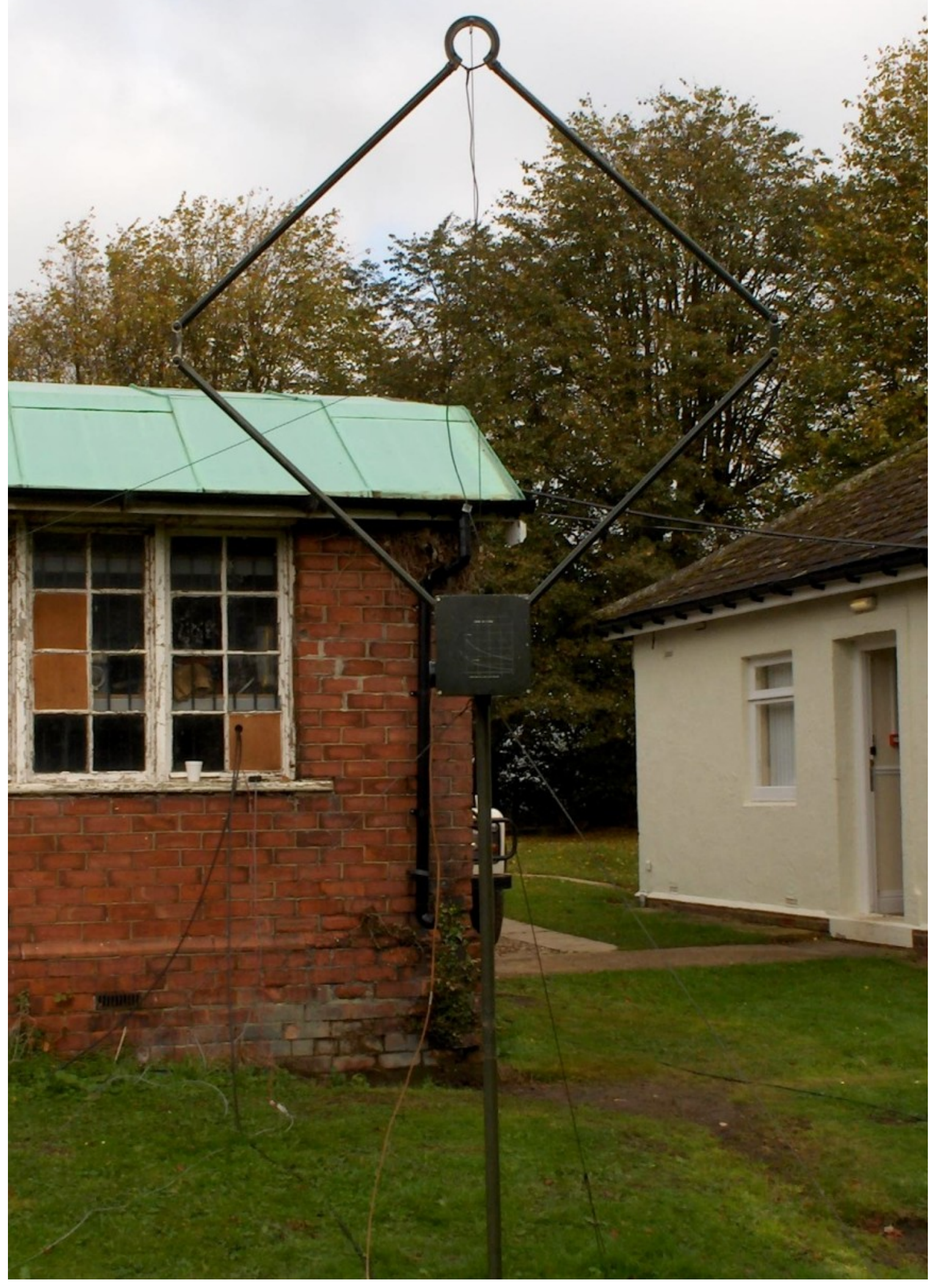

Figure 3.11 Transmitting loop antenna 


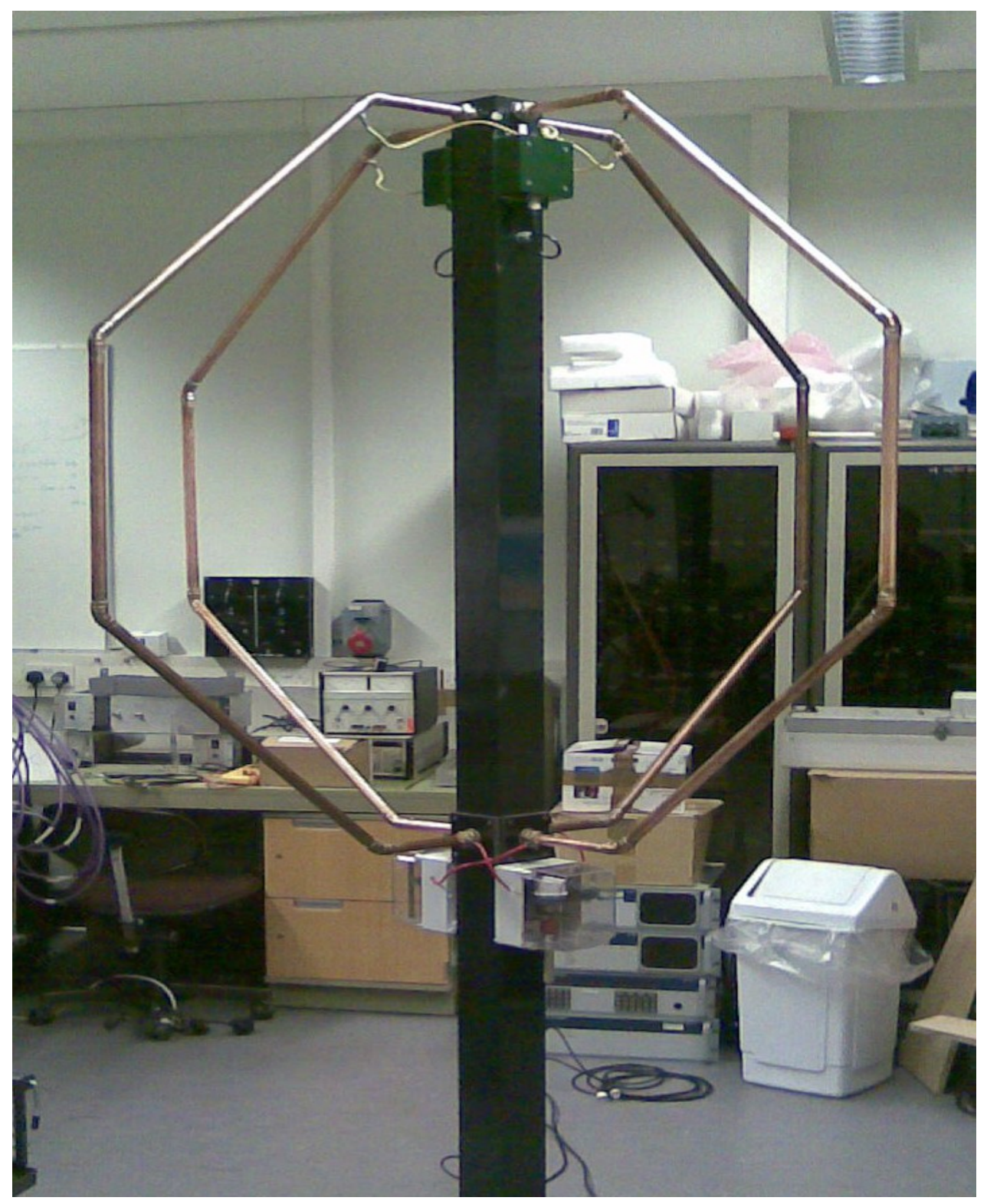

Figure 3.12 Transmitting collocated octagonal loops

\subsection{Receiver System}

For the HF-MIMO experiments a purpose built 8 channel roke manor receiver called Multi-Channel Digital Wideband Receiver (MCDWR) was used. It provides phasecoherent reception of eight channel signals from $0.1 \mathrm{MHz}$ to $30 \mathrm{MHz}$, covering the whole of HF band. RF inputs (50 $\Omega$ ) from the receive antennas were routed into the receiver using the 8 way $\mathrm{D}$ type female sockets. The processed digital data output from 
each receiver is divided into blocks with header containing information about block size, sample rate, receiver frequency etc. This data is transferred to the computer for further processing using the industry standard USB2 cable connection. The control program runs on a windows machine and display options that are available for time domain, frequency domain and polar representations. Access to the receiver is also possible using the API software which allows customisation at the lower level interface.

The receiver is configured for direction finding and beamforming applications. It was programmed to make it work for our HF-MIMO experiments and deployed in all the campaigns used in this project.

For transmission purposes four separate transmitters were used initially, housed in a rack. A four channel HF band transmitter was acquired later to reduce the size of the transmitting system considerably.

\subsection{Experimental Campaigns}

A total of 21 experimental campaigns were carried out spread over two years from September 2007 to August 2009 (Table 3.1). This allowed the data to be collected under a variety of ionospheric conditions. The ionospheric conditions were monitored during each campaign and frequency of transmission varied accordingly. Most of the data was collected at the nominal frequency of 5.255 MHz.

\begin{tabular}{|l|l|l|l|l|l|}
\hline & Date & $\begin{array}{l}\text { Time } \\
\text { (UT) }\end{array}$ & Freq MHz & Rx antennas & Tx antennas \\
\hline 1 & $06 / 09 / 2007$ & $\begin{array}{l}1143- \\
1210\end{array}$ & 6.075 & L-shaped array (LSA) & $\begin{array}{l}\text { 'Deutsche welle', } \\
\text { Rampisham, UK }\end{array}$ \\
\hline $2 a$ & $03 / 10 / 2007$ & $\begin{array}{l}1413- \\
1501\end{array}$ & 6.78 & $\begin{array}{l}\text { LSA + Giselle (Did not } \\
\text { work) }\end{array}$ & Vertical \\
\hline
\end{tabular}




\begin{tabular}{|c|c|c|c|c|c|}
\hline & Date & $\begin{array}{l}\text { Time } \\
\text { (UT) }\end{array}$ & Freq $\mathrm{MHz}$ & Rx antennas & Tx antennas \\
\hline $2 b$ & $03 / 10 / 2007$ & $\begin{array}{l}1507- \\
1601\end{array}$ & 9.04 & $\begin{array}{l}\text { LSA + Giselle (Did not } \\
\text { work) }\end{array}$ & Vertical \\
\hline $2 c$ & $03 / 10 / 2007$ & $\begin{array}{l}1621- \\
1635\end{array}$ & 9.04 & $\begin{array}{l}\text { LSA + Giselle (GSL) (Did not } \\
\text { work) }\end{array}$ & Vertical + Dipole \\
\hline $2 d$ & $03 / 10 / 2007$ & $\begin{array}{l}1635- \\
1655\end{array}$ & 6.78 & LSA + GSL (Did not work) & Vertical + Dipole \\
\hline 3 & $10 / 10 / 2007$ & $\begin{array}{l}1348- \\
1456\end{array}$ & 5.255 & LSA + GSL (Did not work) & Vertical+Loop+Inverted V \\
\hline $4 a$ & $17 / 10 / 2007$ & $\begin{array}{l}1249- \\
1310\end{array}$ & 5.255 & LSA + GSL (Did not work) & 3spaced Dipoles \\
\hline $4 b$ & $17 / 10 / 2007$ & $\begin{array}{l}1331- \\
1418\end{array}$ & 5.255 & LSA + GSL (Did not work) & Vertical+Loop+Inverted V \\
\hline $4 c$ & $17 / 10 / 2007$ & $\begin{array}{l}1445- \\
1537\end{array}$ & 5.255 & LSA + GSL (Did not work) & $\begin{array}{l}\text { Vertical+Loop+ N-S \& E-W } \\
\text { crossed wire Inverted Vs } \\
\text { (CWIVs) }\end{array}$ \\
\hline $5 a$ & $02 / 11 / 2007$ & $\begin{array}{l}1441- \\
1515\end{array}$ & 5.255 & LSA +Loop & 2 CWIVs \\
\hline $5 b$ & $02 / 11 / 2007$ & $\begin{array}{l}1518- \\
1630\end{array}$ & 5.255 & $\begin{array}{l}\text { LSA +Loop+Crossed wire } \\
\text { inverted Vs (CWIVs) }\end{array}$ & 2 CWIVs \\
\hline 6 & $07 / 11 / 2007$ & $\begin{array}{l}1500- \\
1630\end{array}$ & 5.255 & $\begin{array}{l}\mathrm{LSA}(1,2,3,5)+\mathrm{N}, \mathrm{S}, \mathrm{E} \& \mathrm{~W} \\
\text { pointing wire antennas }\end{array}$ & CWIVs \\
\hline $7 a$ & $07 / 12 / 2007$ & $\begin{array}{l}1025- \\
1332\end{array}$ & 5.255 & $\begin{array}{l}\mathrm{LSA}(1,2,3,5)+\mathrm{N}, \mathrm{S}, \mathrm{E} \& \mathrm{~W} \\
\text { pointing wire antennas }\end{array}$ & Octagonal Loop \\
\hline $7 b$ & $07 / 12 / 2007$ & $\begin{array}{l}1033- \\
1100\end{array}$ & 5.255 & $\begin{array}{l}\mathrm{LSA}(1,2,3,5)+\mathrm{N}, \mathrm{S}, \mathrm{E} \& \mathrm{~W} \\
\text { pointing wire antennas }\end{array}$ & Loop+Octagonal Loop \\
\hline $8 a$ & $05 / 06 / 2008$ & $\begin{array}{l}1038- \\
1158\end{array}$ & 5.255 & $\operatorname{LSA}(1,2,3,5)$ & CWIVs+Loop+Vertical \\
\hline $8 b$ & $05 / 06 / 2008$ & $\begin{array}{l}1320- \\
1403\end{array}$ & 5.255 & $\begin{array}{l}\operatorname{LSA}(1,2,3,5)+\text { Active } \\
\text { monopole+CWIVs }\end{array}$ & CWIVs+Loop+Vertical \\
\hline $9 a$ & $16 / 06 / 2008$ & $\begin{array}{l}1231- \\
1439\end{array}$ & 5.255 & $\begin{array}{l}\text { LSA }(1,2,3,5)+\text { Active } \\
\text { monopole+CWIVs }\end{array}$ & CWIVs+Loop+Vertical \\
\hline
\end{tabular}




\begin{tabular}{|c|c|c|c|c|c|}
\hline & Date & $\begin{array}{l}\text { Time } \\
\text { (UT) }\end{array}$ & Freq $\mathrm{MHz}$ & Rx antennas & Tx antennas \\
\hline $9 b$ & $16 / 06 / 2008$ & $\begin{array}{l}1329- \\
1419\end{array}$ & 5.255 & LSA+CWIVs & CWIVs+Loop+Vertical \\
\hline $10 a$ & 01/07/2008 & $\begin{array}{l}1338- \\
1442\end{array}$ & 5.255 & $\begin{array}{l}\text { LSA }(1,3)+C W I V s+3 \\
\text { collocated } \\
\text { monopoles+loop }\end{array}$ & CWIVs+Loop \\
\hline $10 b$ & 01/07/2008 & $\begin{array}{l}1451- \\
1521\end{array}$ & 5.255 & $\begin{array}{l}\text { LSA }(1,3)+C W I V s+3 \\
\text { collocated } \\
\text { monopoles+loop }\end{array}$ & CWIVs+Vertical \\
\hline $11 a$ & 02/07/2008 & $\begin{array}{l}1002- \\
1153\end{array}$ & 5.255 & $\begin{array}{l}\text { LSA }(1,3)+C W I V s+3 \\
\text { collocated } \\
\text { monopoles+loop }\end{array}$ & CWIVs+Loop+Vertical \\
\hline $11 b$ & $02 / 07 / 2008$ & $\begin{array}{l}1208- \\
1429\end{array}$ & 4.4455 & $\begin{array}{l}\text { LSA }(1,3)+C W I V s+3 \\
\text { collocated } \\
\text { monopoles+loop }\end{array}$ & CWIVs+Loop+Vertical \\
\hline $11 c$ & 02/07/2008 & $\begin{array}{l}1444- \\
1536\end{array}$ & 3.95 & $\begin{array}{l}\text { LSA }(1,3)+C W I V s+3 \\
\text { collocated } \\
\text { monopoles+loop }\end{array}$ & CWIVs+Vertical \\
\hline $12 a$ & 03/07/2008 & $\begin{array}{l}1016- \\
1422\end{array}$ & 5.795 & $\begin{array}{l}\mathrm{LSA}(1,3)+\mathrm{CWIVs}+3 \\
\text { collocated } \\
\text { monopoles+loop }\end{array}$ & CWIVs+Loop+Vertical \\
\hline $12 b$ & 03/07/2008 & $\begin{array}{l}1443- \\
1509\end{array}$ & 5.255 & $\begin{array}{l}\mathrm{LSA}(1,3)+\mathrm{CWIVs}+3 \\
\text { collocated } \\
\text { monopoles+loop }\end{array}$ & CWIVs+Loop+Vertical \\
\hline $13 a$ & 29/07/2008 & $\begin{array}{l}1151- \\
1306\end{array}$ & 5.255 & $\begin{array}{l}\text { CWIVs+3 collocated } \\
\text { monopoles }+X Y Z \text { loop array }\end{array}$ & CWIVs+Loop+Vertical \\
\hline $13 b$ & 29/07/2008 & $\begin{array}{l}1318- \\
1419\end{array}$ & 4.4455 & $\begin{array}{l}\text { CWIVs+3 collocated } \\
\text { monopoles }+X Y Z \text { loop array }\end{array}$ & CWIVs+Loop+Vertical \\
\hline $14 a$ & 07/08/2008 & $\begin{array}{l}1105- \\
1140\end{array}$ & 5.255 & $\begin{array}{l}\text { CWIVs+3 collocated } \\
\text { monopoles+XYZ loop array }\end{array}$ & CWIVs+Loop+Vertical \\
\hline $14 b$ & 07/08/2008 & $\begin{array}{l}1158- \\
1357\end{array}$ & 5.255 & $\begin{array}{l}\text { CWIVs+ LSA }(1,2,3)+\mathrm{XYZ} \\
\text { loop array }\end{array}$ & CWIVs+Loop+Vertical \\
\hline $15 a$ & 03/09/2008 & $\begin{array}{l}1008- \\
1213\end{array}$ & 5.255 & $\begin{array}{l}\text { NS inverted } \mathrm{V}+\text { Loop }+3 \\
\text { collocated monopoles }+\mathrm{XYZ}\end{array}$ & CWIVs+Loop+Vertical \\
\hline
\end{tabular}




\begin{tabular}{|c|c|c|c|c|c|}
\hline & Date & $\begin{array}{l}\text { Time } \\
\text { (UT) }\end{array}$ & Freq $\mathrm{MHz}$ & Rx antennas & Tx antennas \\
\hline & & & & loop array & \\
\hline $15 b$ & 03/09/2008 & $\begin{array}{l}1251- \\
1422\end{array}$ & 5.255 & $\begin{array}{l}\text { NS inverted } \mathrm{V}+\text { Loop }+3 \\
\text { collocated monopoles }+\mathrm{XYZ} \\
\text { loop array (collocated } \\
\text { north-south, east-west and } \\
\text { horizontal loops) }\end{array}$ & $\begin{array}{l}\text { CWIVs+Loop+Octagonal } \\
\text { Loop }\end{array}$ \\
\hline $16 a$ & $09 / 12 / 2008$ & $\begin{array}{l}1301- \\
1329\end{array}$ & 5.255 & $\begin{array}{l}\text { Crossed dipoles+GSL } \\
\text { array+XYZ loop array }\end{array}$ & CWIVs+Loop \\
\hline $16 b$ & $09 / 12 / 2008$ & $\begin{array}{l}1335- \\
1341\end{array}$ & 5.255 & $\begin{array}{l}\text { Crossed dipoles+ GSL } \\
\text { array+XYZ loop array }\end{array}$ & $\begin{array}{l}\text { CWIVs+Loop+Octagonal } \\
\text { Loop1 }\end{array}$ \\
\hline $16 c$ & $09 / 12 / 2008$ & $\begin{array}{l}1357- \\
1415\end{array}$ & 5.255 & $\begin{array}{l}\text { Crossed dipoles+ GSL } \\
\text { array+XYZ loop array }\end{array}$ & $\begin{array}{l}\text { CWIVs+Loop+Octagonal } \\
\text { Loop2 }\end{array}$ \\
\hline $16 d$ & $09 / 12 / 2008$ & $\begin{array}{l}1458- \\
1513\end{array}$ & 5.255 & $\begin{array}{l}\text { Crossed dipoles+ GSL } \\
\text { array+XYZ loop array }\end{array}$ & $\begin{array}{l}\text { CWIVs+Loop+Collocated } \\
\text { Octagonal Loops }\end{array}$ \\
\hline $17 a$ & $29 / 01 / 2009$ & $\begin{array}{l}1258- \\
1357\end{array}$ & 5.255 & $\begin{array}{l}\text { CWIVs+ GSL array+XYZ } \\
\text { loop array }\end{array}$ & CWIVs \\
\hline $17 b$ & $29 / 01 / 2009$ & $\begin{array}{l}1408- \\
1438\end{array}$ & 5.255 & $\begin{array}{l}\text { Crossed dipoles+ GSL } \\
\text { array+XYZ loop array }\end{array}$ & CWIVs \\
\hline $18 \mathrm{a}$ & $26 / 03 / 2009$ & $\begin{array}{l}1100- \\
1213\end{array}$ & 4.4455 & $\begin{array}{l}\text { CWIVs+Crossed dipoles } \\
\text { with monopole+XYZ loop } \\
\text { array }\end{array}$ & $\begin{array}{l}\text { CWIVs +Octagonal Loop } \\
\text { (EW) }\end{array}$ \\
\hline $18 \mathrm{~b}$ & $26 / 03 / 2009$ & $\begin{array}{l}1234- \\
1318\end{array}$ & 4.4455 & $\begin{array}{l}\text { CWIVs +Crossed dipoles } \\
\text { with monopole+XYZ loop } \\
\text { array }\end{array}$ & $\begin{array}{l}\text { CWIVs +Octagonal Loop } \\
\text { (NS) }\end{array}$ \\
\hline $18 \mathrm{c}$ & $26 / 03 / 2009$ & $\begin{array}{l}1347- \\
1455\end{array}$ & 4.455 & $\begin{array}{l}\text { CWIVs +Crossed dipoles } \\
\text { with monopole+XYZ loop } \\
\text { array }\end{array}$ & $\begin{array}{l}\text { CWIVs +collocated } \\
\text { Octagonal Loops }\end{array}$ \\
\hline $19 a$ & $19 / 06 / 2009$ & $\begin{array}{l}1135- \\
1218\end{array}$ & 5.255 & $\begin{array}{l}\text { CWIVs+ GSL array+XYZ } \\
\text { loop array }\end{array}$ & CWIVs \\
\hline $19 b$ & $19 / 06 / 2009$ & $\begin{array}{l}1243- \\
1311\end{array}$ & 4.455 & $\begin{array}{l}\text { CWIVs+ GSL array+XYZ } \\
\text { loop array }\end{array}$ & $\begin{array}{l}\text { CWIVs (BPSK+no offset, did } \\
\text { not work) }\end{array}$ \\
\hline $19 \mathrm{c}$ & $19 / 06 / 2009$ & 1317- & 4.4455 & CWIVs+ GSL array+XYZ & CWIVs (BPSK+no offset, did \\
\hline
\end{tabular}




\begin{tabular}{|c|c|c|c|c|c|}
\hline & Date & $\begin{array}{l}\text { Time } \\
\text { (UT) }\end{array}$ & Freq $\mathrm{MHz}$ & Rx antennas & Tx antennas \\
\hline & & 1343 & & loop array & not work) \\
\hline $20 a$ & $23 / 07 / 2009$ & $\begin{array}{l}1131- \\
1218\end{array}$ & 4.4455 & $\begin{array}{l}\text { CWIVs+ GSL array+XYZ } \\
\text { loop array }\end{array}$ & $\begin{array}{l}\text { CWIVs +Octagonal Loop } \\
\text { (EW) }\end{array}$ \\
\hline $20 \mathrm{~b}$ & 23/07/2009 & $\begin{array}{l}1230- \\
1303\end{array}$ & 4.4455 & $\begin{array}{l}\text { CWIVs+ GSL array+XYZ } \\
\text { loop array }\end{array}$ & $\begin{array}{l}\text { CWIVs +Octagonal Loop } \\
\text { (NS) }\end{array}$ \\
\hline $20 c$ & $23 / 07 / 2009$ & $\begin{array}{l}1334- \\
1340\end{array}$ & 4.4455 & $\begin{array}{l}\text { CWIVs+ GSL array+XYZ } \\
\text { loop array }\end{array}$ & $\begin{array}{l}\text { CWIVs (BPSK+no offset, } \\
\text { did not work) }\end{array}$ \\
\hline $20 d$ & $23 / 07 / 2009$ & $\begin{array}{l}1343- \\
1349\end{array}$ & 4.4455 & $\begin{array}{l}\text { CWIVs+ GSL array+XYZ } \\
\text { loop array }\end{array}$ & $\begin{array}{l}\text { CWIVs (BPSK+no offset, did } \\
\text { not work) }\end{array}$ \\
\hline $20 \mathrm{e}$ & $23 / 07 / 2009$ & $\begin{array}{l}1351- \\
1359\end{array}$ & 4.4455 & $\begin{array}{l}\text { CWIVs+ GSL array+XYZ } \\
\text { loop array }\end{array}$ & $\begin{array}{l}\text { CWIVs +Octagonal Loop } \\
\text { (NS) (BPSK+no offset, did } \\
\text { not work) }\end{array}$ \\
\hline $20 f$ & 23/07/2009 & $\begin{array}{l}1404- \\
1410\end{array}$ & 4.4455 & $\begin{array}{l}\text { CWIVs+ GSL array+XYZ } \\
\text { loop array }\end{array}$ & $\begin{array}{l}\text { CWIVs +Octagonal Loop } \\
\text { (NS) (BPSK+no offset, did } \\
\text { not work) }\end{array}$ \\
\hline $21 a$ & $11 / 08 / 2011$ & $\begin{array}{l}1247- \\
1336\end{array}$ & 5.255 & $\begin{array}{l}\text { CWIVs+ GSL array+XYZ } \\
\text { loop array }\end{array}$ & $\begin{array}{l}\text { CWIVs +collocated } \\
\text { Octagonal Loops }\end{array}$ \\
\hline $21 b$ & $11 / 08 / 2011$ & $\begin{array}{l}1343- \\
1436\end{array}$ & 5.255 & $\begin{array}{l}\text { CWIVs+ GSL array+XYZ } \\
\text { loop array }\end{array}$ & $\begin{array}{l}\text { CWIVs +collocated } \\
\text { Octagonal Loops }\end{array}$ \\
\hline
\end{tabular}

Table 3.1 The detail of campaigns carried out in HF-MIMO study

\subsection{Data Processing}

Since continuous wave (CW) signals were used in the campaigns the transmitters were fed with an offset of $10 \mathrm{~Hz}$. This allowed for the extraction of the signals from the different transmitters at the receiver. It was ensured in a test campaign that the $10 \mathrm{~Hz}$ difference will not cause the channel conditions to change. The data from the receiver was ported into the computer and then analyzed using MATLAB. Each receiving antenna signal consisted of streams from multiple transmitters which were then filtered to extract individual signals. Different filters were tested on the data set but the square 
filter was finally selected as it gave the most consistent results. Correlation coefficients were then computed for each antenna pair at both the transmitter and the receiver from the filtered data. Finally the capacity estimates were calculated using the Equation 2.1. The channel matrix in the capacity equation was formed using the signal peaks of the Fourier transform of the signals for each transmit receive pair (Figure 3.13) for each measurement. The normalization of channel matrix allows for the comparison between antennas with different received powers. Different methods of normalization of the channel matrix could be adapted in order to calculate capacity but the meaning of SNR will change with the method selected [Loyka and Levin, 2009]. In this case the channel matrices were normalized by making the average power transfer between each transmit-receive pair to be one i.e. the SISO gain of 1 [Wallace et al., 2003]. This allows the capacity comparisons to be based on MIMO parameters rather than the

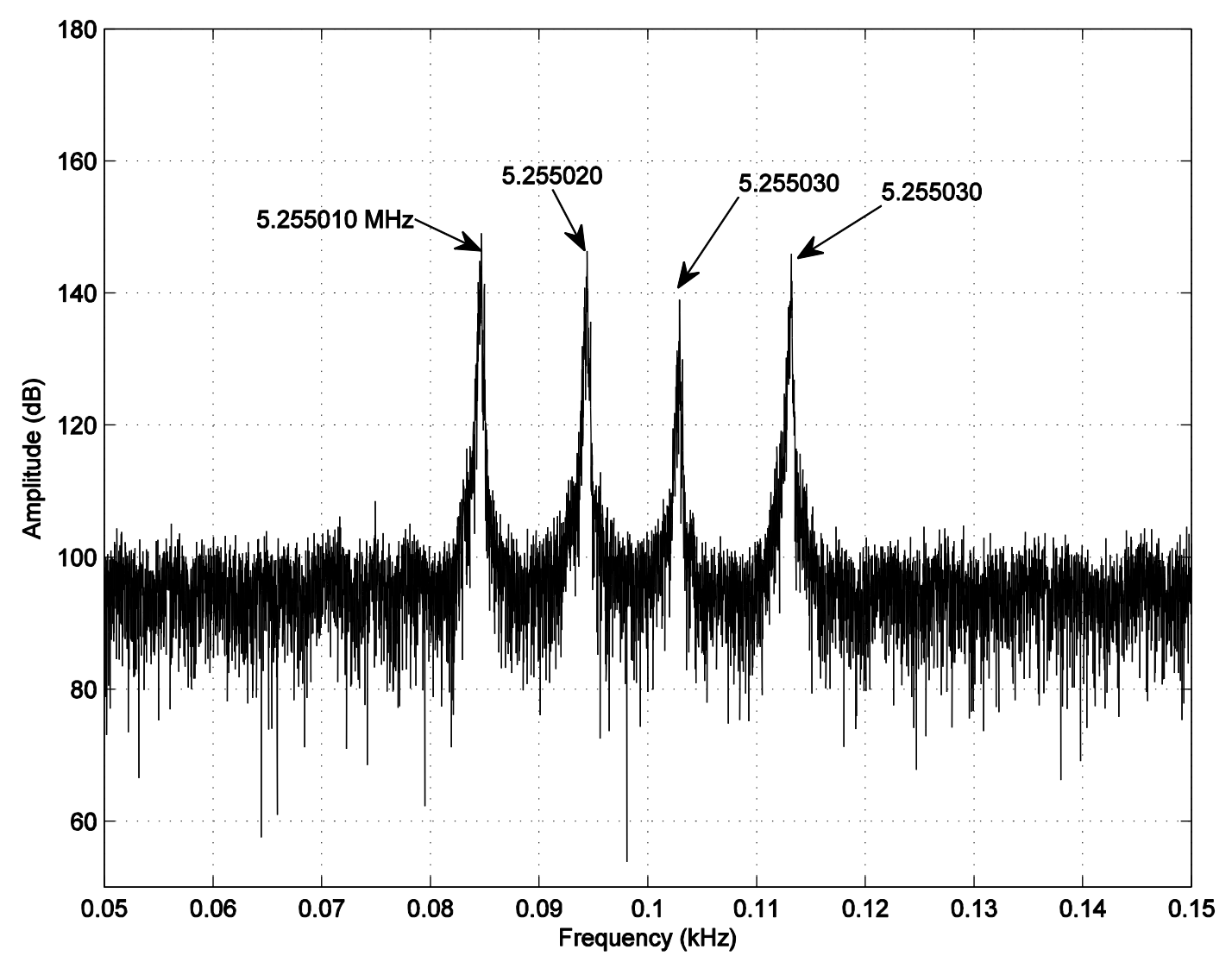

Figure 3.13 Sample FFT graph of four trans mit signals $10 \mathrm{~Hz}$ apart 
power gains. To normalize the channel matrix Frobenius norm is calculated by summing the absolute squares of the channel matrix entries and taking the square root. The square of the Frobenius norm may be used to represent the total power gain of the channel, the statistics of which determine the diversity performance [Paulraj et al., 2003]. The normalization factor is then multiplied with the channel matrix obtained to get the normalized channel matrix.

$$
H_{\text {normalized }}=H\left[\frac{1}{n_{R} n_{T}} \sum_{i=1}^{n_{R}} \sum_{j=1}^{n_{T}}\left|H_{i j}\right|^{2}\right]^{-1 / 2}
$$




\section{Measurements using spaced antenna arrays in HF-MIMO}

Spaced arrays rely on the separation distance in terms of the wavelength to introduce decorrelation between the signals at the antennas. Such arrays were a natural choice when MIMO concepts were being investigated. Increasing the separation increases the phase difference and hence the decorrelation between them. Also the increase in distance between scatterer's increases the decorrelation at a fixed antenna separation. Collocated arrays are arranged on a common coordinate axis and thus cannot decorrelate the signals spatially. Thus in order to use such arrays for MIMO, diversity has to be achieved by some other means.

\subsection{Correlation measurements using the L-shaped spaced array}

Initial measurements employed a spaced array of monopole antennas as shown in Figure 3.2. This separation distances between the antennas (13.3 m, $26.7 \mathrm{~m}$ and $40 \mathrm{~m})$ translated into the wavelength distances of $0.22 \lambda, 0.45 \lambda$ and $0.67 \lambda$ respectively in terms of the nominal frequency of $5 \mathrm{MHz}$ used in most of the campaigns. This gave a separations of less than, greater than and approximately equal to the $\lambda / 2$.

\subsubsection{A comparison of separation distance and orientation dependent effects on correlation}

The campaign for this discussion was carried out on 02 November 2007 . Four transmit antennas were used which consisted of two pairs of crossed wire inverted V antennas with a spacing of $22 \mathrm{~m}$ between them. The north-south arm of each pair was pointing towards Durham and the other arm was oriented orthogonal to it in the east-west direction. The frequencies used were $5.255010 \mathrm{MHz}(\mathrm{Tx} 1, \mathrm{E}-\mathrm{W}$ antenna of crossed wire 1), 5.255020 MHz (Tx2, N-S antenna of crossed wire 1), 5.255030 MHz (Tx3, E- 
W antenna of crossed wire 2) and 5.255040 MHz (N-S antenna of crossed wire 2). The data was collected between 1445 UT to 1818 UT and consisted of 1 minute duration data acquisitions. The representative ionograms during this period from Chilton ionosonde are shown in the Figure 4.1. The ionograms show the presence of multiple propagation modes. Both $\mathrm{O}$ and $\mathrm{X}$ modes can be observed in all ionograms. Multiple reflections from both $\mathrm{E}$ and $\mathrm{F}$ region can also be identified. The amplitude $\mathrm{v}$ time graphs at receive antennas also show the presence of fading with lows at un-identical positions. An example plot is given in Figure 4.2 for the receive antennas 1-4. The magnitude of the signal varies for different transmit antennas however the fading patterns can be seen to be quite independent.
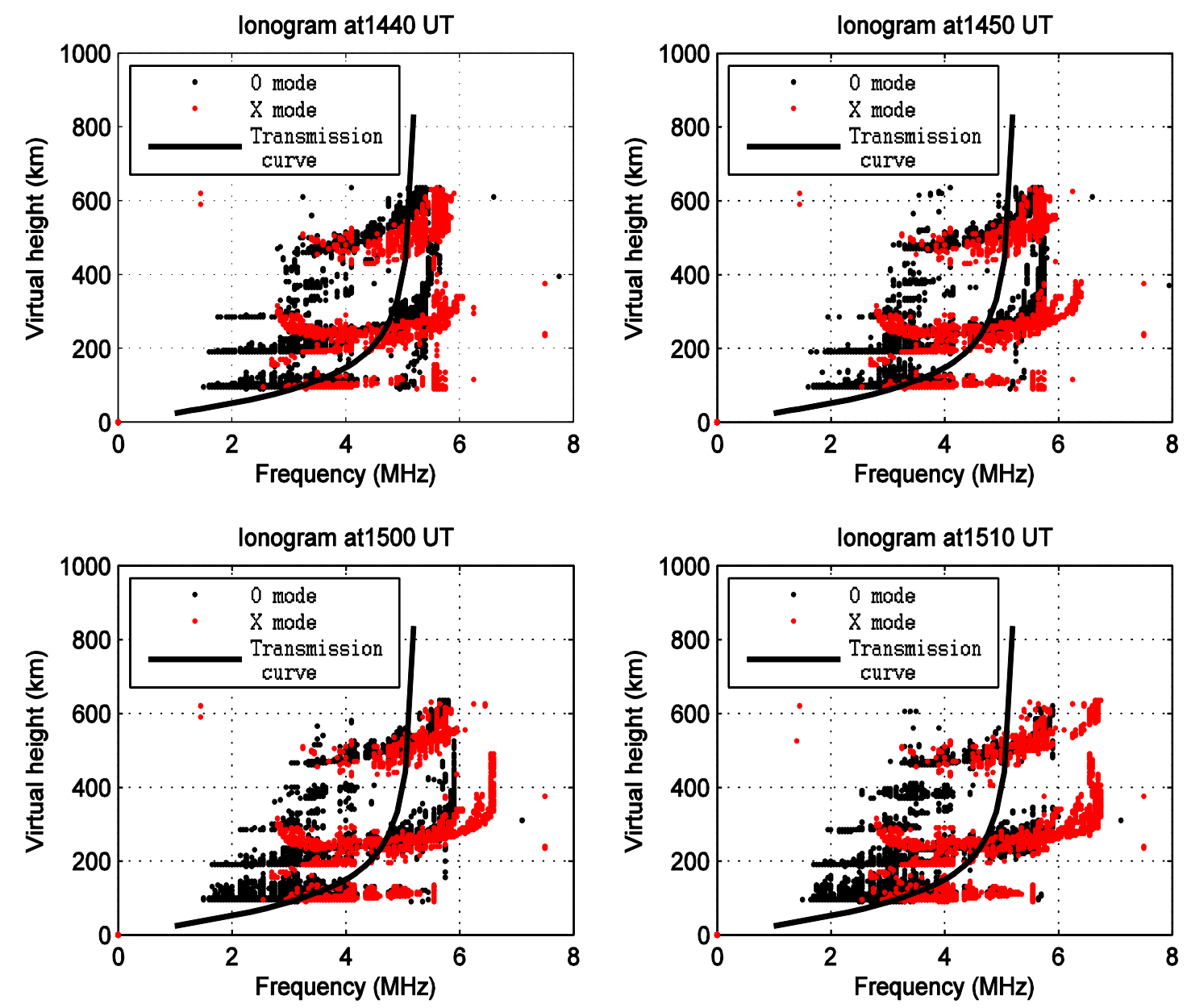

Figure 4.1 The ionograms with transmission curve overlaid, obtained from Chilton ionosonde for the campaign on 02 Nove mber 2007 
Envelope correlation coefficients were computed for the one minute measurements at both the transmit and receive antenna pairs and stored in bins with the range of 0.1 . Traditionally an envelope correlation coefficient of 0.7 or less is considered to be adequate to provide diversity reception [Jakes, 1974]. An experimental study on a uniform linear array however asserts that the inter-element correlation coefficient of less than 0.9 is sufficient to provide the MIMO capacity improvements [Loyka, 2001]. Figure 4.3 shows the envelope correlation coefficients for antenna pairs 1 and 2, and 1 and 3 of the north-south arm of the $\mathrm{L}$ shaped homogenous array. In the case for the signal from $\mathrm{Tx} 4,74 \%$ and $24 \%$ of correlation coefficient values lie below 0.9 and 0.7 respectively for the monopole pair with a separation of $26.7 \mathrm{~m}$. The antennas 1 and 3 with a separation distance of $40 \mathrm{~m}$ have $83 \%$ and $57 \%$ values below 0.9 and 0.7 respectively which is appreciably higher than the previous pair. The difference is higher for other transmit antennas. Thus as expected the correlation coefficients between antennas in a homogenous spaced array will depend on the distance that separates these antennas.

The results proves the assertion found in literature that in order to achieve significant decorrelation antennas must have a separation distance of at-least $\lambda / 2$ between them [Jakes, 1974]. In this case we have a separation distance of less than $\lambda / 2$ between antennas $1 \& 2$ and more than $\lambda / 2$ between antennas $1 \& 3$. Also diversity at the receive antennas will depend on the type of antenna used at the transmitter. The orientation dependent effects on the envelope correlation coefficients can be seen in the Figure 4.4 when compared with Figure 4.3. In this figure the east-west arm of the homogenous array has been used. The correlation coefficients computed for signal from Tx1 reveal that only $17 \%$ and $24 \%$ of the values lie below 0.9 for the pairs with a separation of $26.7 \mathrm{~m}$ and $40 \mathrm{~m}$ respectively. Thus the north-south oriented antennas 
are much more de-correlated than the east-west oriented antennas with the same spacing.
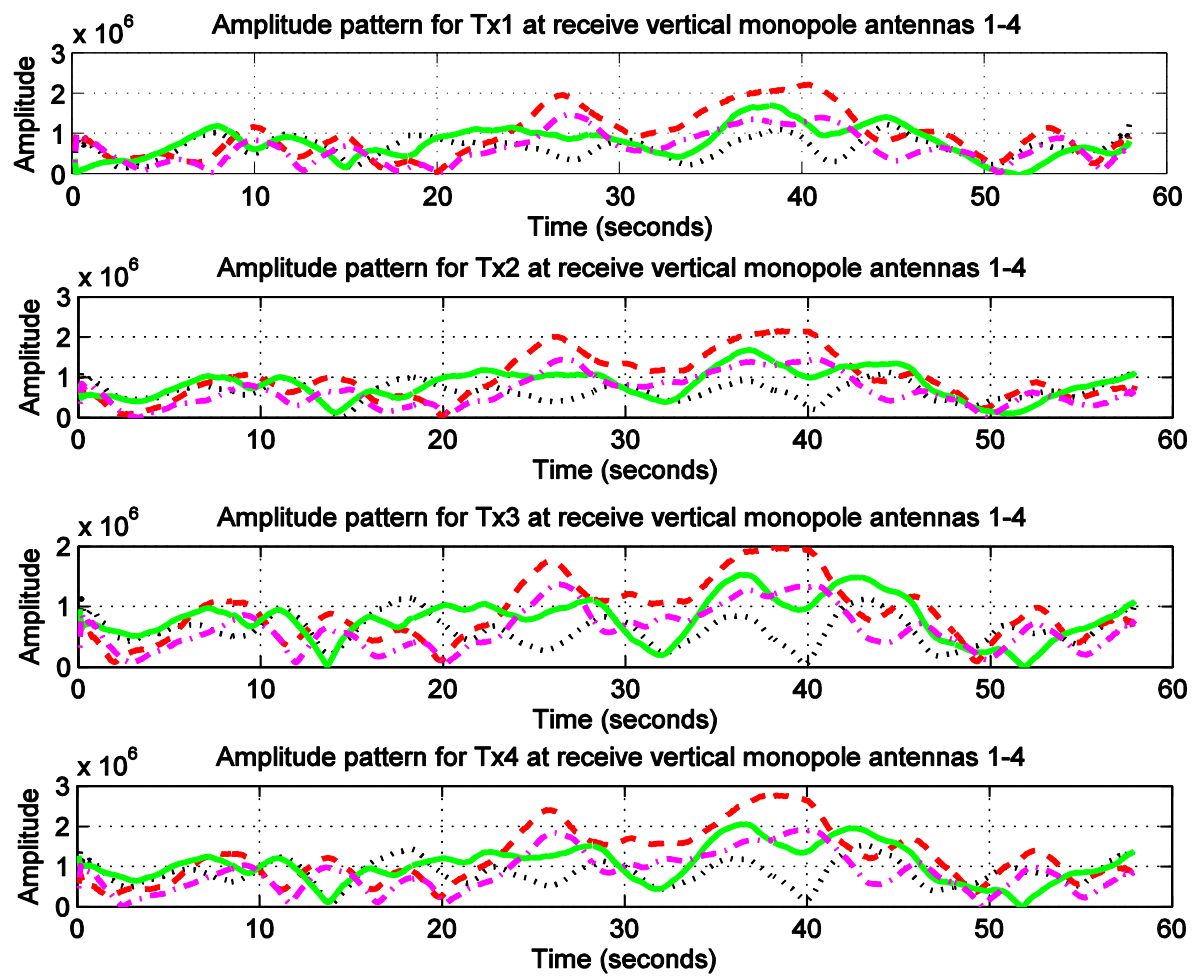

Figure 4.2 An example amplitude pattern for $T x$ antennas at the first $4 \mathrm{Rx}$ antenna for the meas ure ment obtained at 1610 UT on 02 Nove mber 2007

Also we see that the correlation between antennas does not fall off appreciably when the separation distance is increased to a value greater than the $\lambda / 2$. It is known that the correlation between antennas is dependent on the azimuthal angle of arrival of the signal and the relative angle between the positions of the antennas [Lee, 1973]. Thus the spaced antennas arranged in line with the direction of transmission would decorrelate for smaller separation distances than the ones arranged in a broadside array. This could be due to the fact that the impinging radio waves coming from the north would have to travel comparatively greater distances (therefore resulting in more decorrelation) compared to the east-west arm. It has also been shown in the literature that for line of sight communication the correlation drops down considerably with distance in endfire arrays as compared to the broadside arrays [Malik, 2008]. 

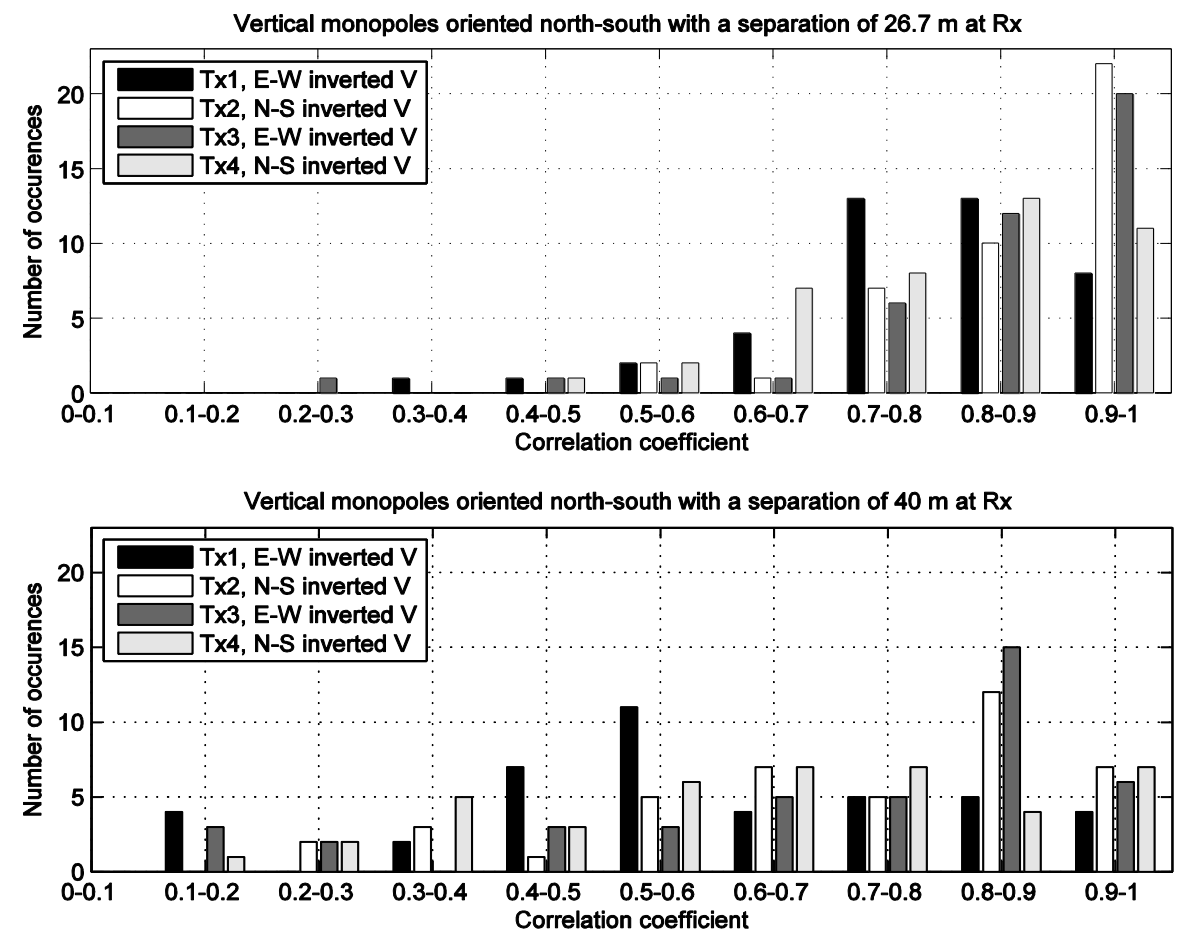

Figure 4.3 Envelope correlation coefficient histograms for s paced antennas $1 \& 2$ (top) and $1 \& 3$ (bottom)
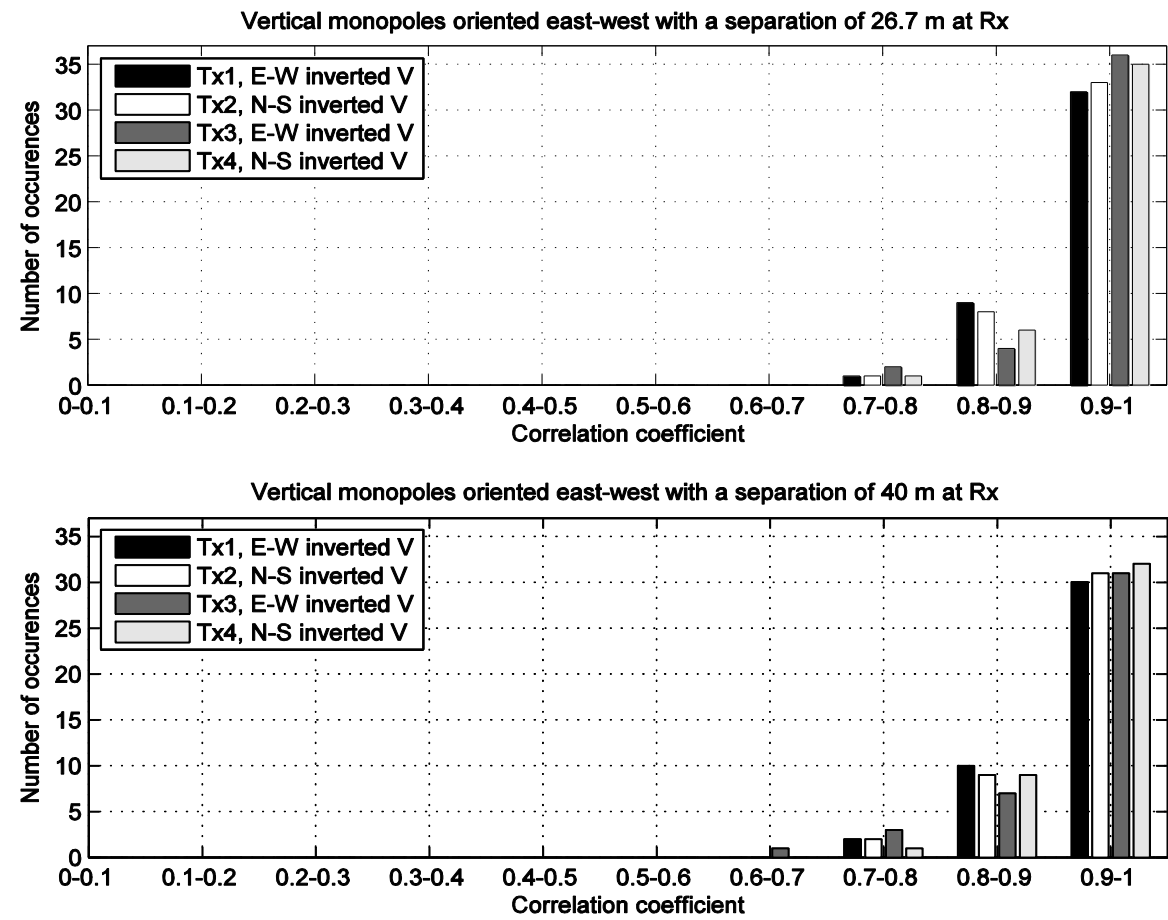

Figure 4.4 Envelope correlation coefficient histograms for spaced antenn as $4 \& 5$ (top) and $3 \& 5$ (bottom) 


\subsubsection{A comparison of separation distance effects on correlation}

In another campaign carried out on 7 August 2008 we analysed the results for another set of propagation conditions for the same L-shaped array at the receiver taking into consideration the north-south oriented arm (antennas 1, 2 \& 3). Four transmit antennas were used which consisted of the collocated crossed wire inverted Vs (north-south and east-west), Racal loop and a vertical. Again the nominal frequency of $5.255 \mathrm{MHz}$ was used with a separation of $10 \mathrm{~Hz}$ at each transmitter. The data was collected between 1158 UT to 1357 UT and consisted of 1 minute duration data acquisitions. The representative ionograms during this period from Chilton ionosonde are shown in the Figure 4.6. The ionograms are less dense with $\mathrm{E}$ and $\mathrm{F}$ region reflections. Hence less number of multipath components will be available at the receiver end. The amplitude patterns at receive antennas show that the fading patterns at the 3 antennas are less independent and the signals at the receiving antennas are more correlated compared to the previous campaign. The antennas with a spacing of $13.3 \mathrm{~m}$ and $26.7 \mathrm{~m}$ are not able to generate a high level of decorrelation. For $\mathrm{Tx} 2$ the correlation coefficients stay below 0.9 and 0.7 for $21 \%$ and $3 \%$ when the pair with spacing of $13.3 \mathrm{~m}$ is used. Increasing the separation distance to $26.7 \mathrm{~m}$ increases the number of correlation coefficient values below 0.9 to $32 \%$ while the number of values below 0.7 stay the same at $3 \%$. The antennas with a separation of $40 \mathrm{~m}$ perform better and are more decorrelated. $45 \%$ and $3 \%$ of the correlation coefficients values lie below 0.9 and 0.7 respectively for $\mathrm{T} \times 2$. Thus a gradual increase in the decorrelation is witnessed as the separation distance is increased between individual antennas in the array.

Comparing the results of this campaign with the one previously described we see that the ionospheric conditions have a marked effect on the same set of spaced antennas and the decorrelation provided varies. However, whatever the ionospheric 
conditions the increase in spacing will result in somewhat better performance in terms of decorrelation. Also the decorrelation between similar sensors at the same separation distance will be different depending on the manner in which the antennas are oriented with respect to the angle of arrival of the incoming signal. The summary of the results of all campaigns for the L-shaped spaced array for different separation distances is shown in Table 4.1. It is evident that the correlation between antenna pairs decreases
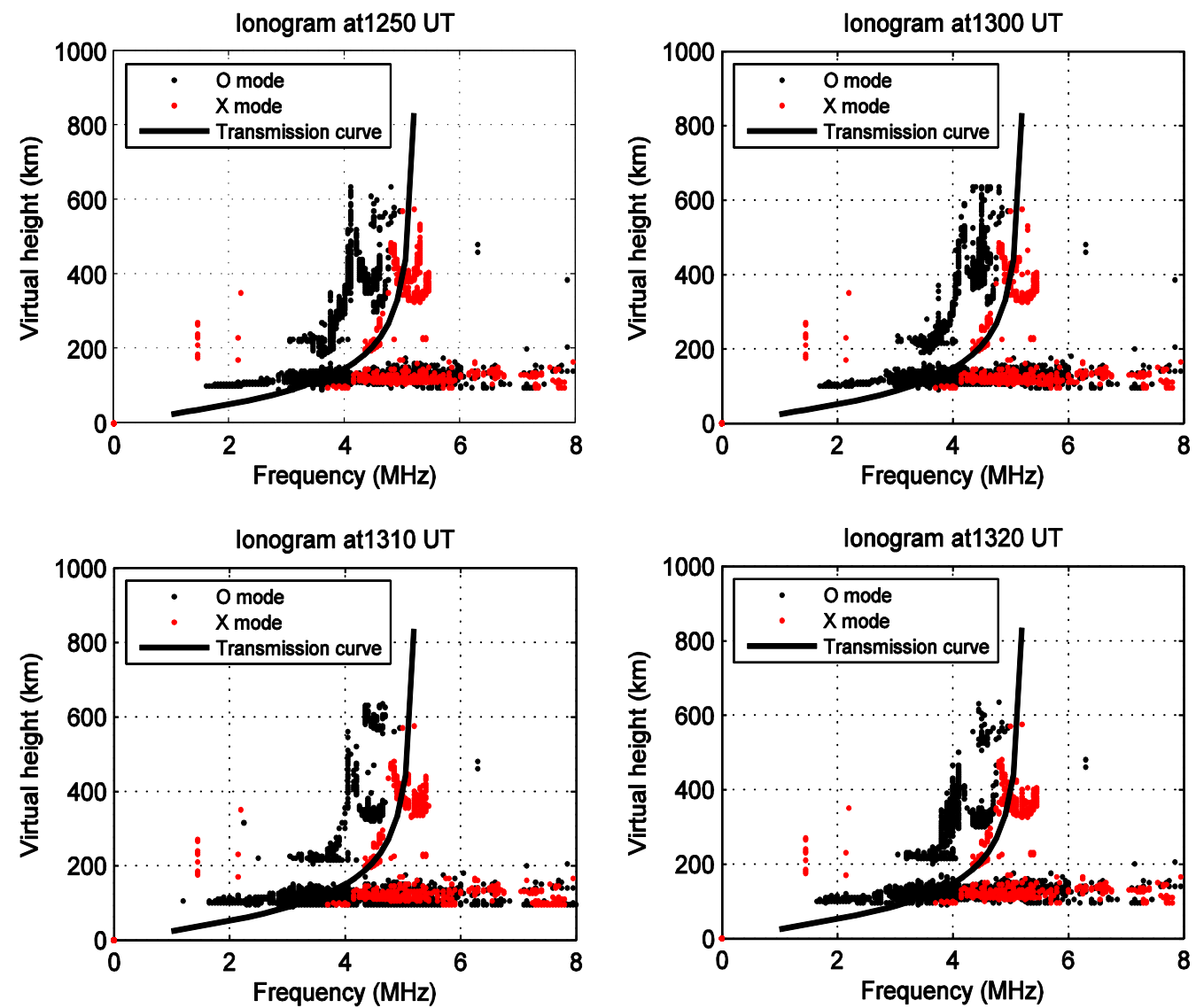

Figure 4.5 The ionograms with transmission curve overlaid, obtained from Chilton ionosonde for the campaign on 7th August 2008

\begin{tabular}{|c|c|c|c|c|c|}
\hline & Separation & $\begin{array}{c}\text { Average } \\
\text { correlation }\end{array}$ & $\begin{array}{c}\text { Standard } \\
\text { deviation }\end{array}$ & \%values $<0.9$ & \%values $<0.7$ \\
\hline \multirow{2}{*}{$\begin{array}{c}\text { East-West } \\
\text { orientation }\end{array}$} & $13.3 \mathrm{~m}$ & 0.90 & 0.20 & 20 & 7 \\
\cline { 2 - 6 } & $27.7 \mathrm{~m}$ & 0.88 & 0.12 & 39 & 8 \\
\cline { 2 - 6 } & $40 \mathrm{~m}$ & 0.73 & 0.30 & 60 & 36 \\
\hline \multirow{2}{*}{$\begin{array}{c}\text { North-South } \\
\text { orientation }\end{array}$} & $13.3 \mathrm{~m}$ & 0.83 & 0.22 & 38 & 14 \\
\cline { 2 - 6 } & $27.7 \mathrm{~m}$ & 0.78 & 0.19 & 70 & 27 \\
\cline { 2 - 6 } & $40 \mathrm{~m}$ & 0.74 & 0.25 & 70 & 38 \\
\hline
\end{tabular}

Table 4.1 Summary of the results for the L-shaped spaced array 
with the increase in distance. Also the north-south arm of the array performs better than the east-west arm generally.

\subsection{Collocated and heterogeneous arrays}

The collocation allows for the placement of the MIMO antennas in the area of a single antenna dimensions. Thus no additional space requirements are needed for deploying MIMO antennas.

\subsubsection{Experiment with the crossed wire inverted $V$ antennas and the significance of ionospheric conditions}

In a campaign carried out on 01 July 2008 we selected the collocated inverted V antennas along with the L-shaped array (antennas 1 \&3) for this analysis (see Figure 4.6). The central support pole of crossed wire was at $49 \mathrm{~m}$ from vertical monopole 2 . At the transmitter similar collocated inverted $\mathrm{V}$ antennas were used. A nominal frequency of 5.255 MHz was used with a separation of $10 \mathrm{~Hz}$ at each transmitter. The

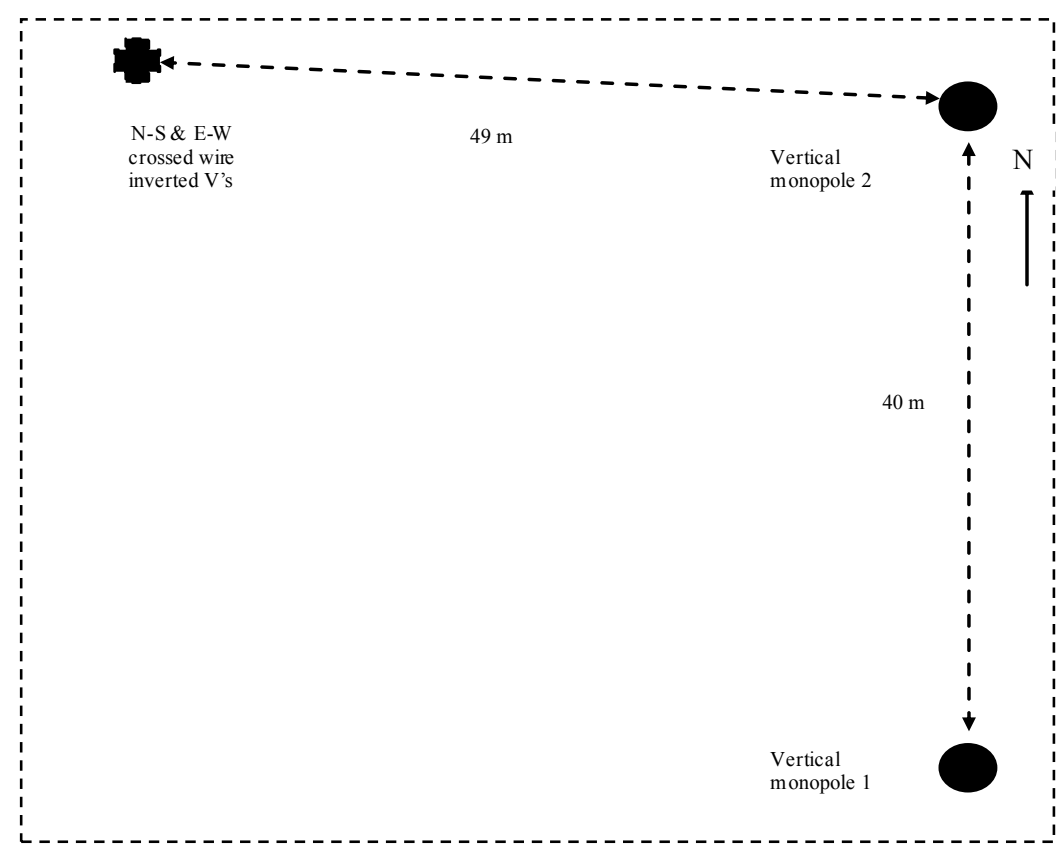

Figure 4.6 Antenna configuration at the receiver on 01 July 2008 
data were collected in two parts and analyzed separately as different ionospheric conditions occurred.

In the first part of this campaign data were collected between 1347 UT to 1447 UT. The ionograms during this period from Chilton ionosonde with transmission curves at the nominal frequency of $5.255 \mathrm{MHz}$ are shown in Figure 4.7. The ionograms show the reflections from the $\mathrm{E}$ region are dominant and the $\mathrm{F}$ layer reflections are predominantly for $\mathrm{X}$ mode. It can also be observed that the transmission curve skips the $\mathrm{F}$ region for $\mathrm{O}$ mode completely. There are $\mathrm{F}$ region reflections but there is not much of the multipath available for the frequency used since multiple hop propagation is negligible. Hence limited number of multipath components will be available at the receiver end. The amplitude patterns at receive antennas show that the fading for the two monopoles is almost identical. The envelope correlation coefficients were computed from the acquired data. The spaced monopoles were found to be highly correlated with all values above 0.9 . This shows that under such conditions spaced homogenous array with spacing of $40 \mathrm{~m}(>\lambda / 2)$ will have limited advantage and the capacity improvements due to the receive antenna decorrelation will be negligible. Thus in order to achieve some decorrelation the spacing between the antennas has to be increased further. Increasing it to $\lambda$ means that $60 \mathrm{~m}$ of a space requirement at the frequency of $5 \mathrm{MHz}$. On the other hand the crossed wire pair depicts certain level of decorrelation. Around $50 \%$ of the correlation coefficient values lie below the 0.9. As MIMO channel capacity does not degrade significantly until the inter-element correlation exceeds approximately 0.9 [Loyka, 2001], the crossed wire antennas will contribute to the capacity enhancement under the prevailing ionospheric conditions. On the transmit site at Durham crossed wire inverted $\mathrm{V}$ antennas were used. The correlation coefficient values for these inverted $\mathrm{V}$ 

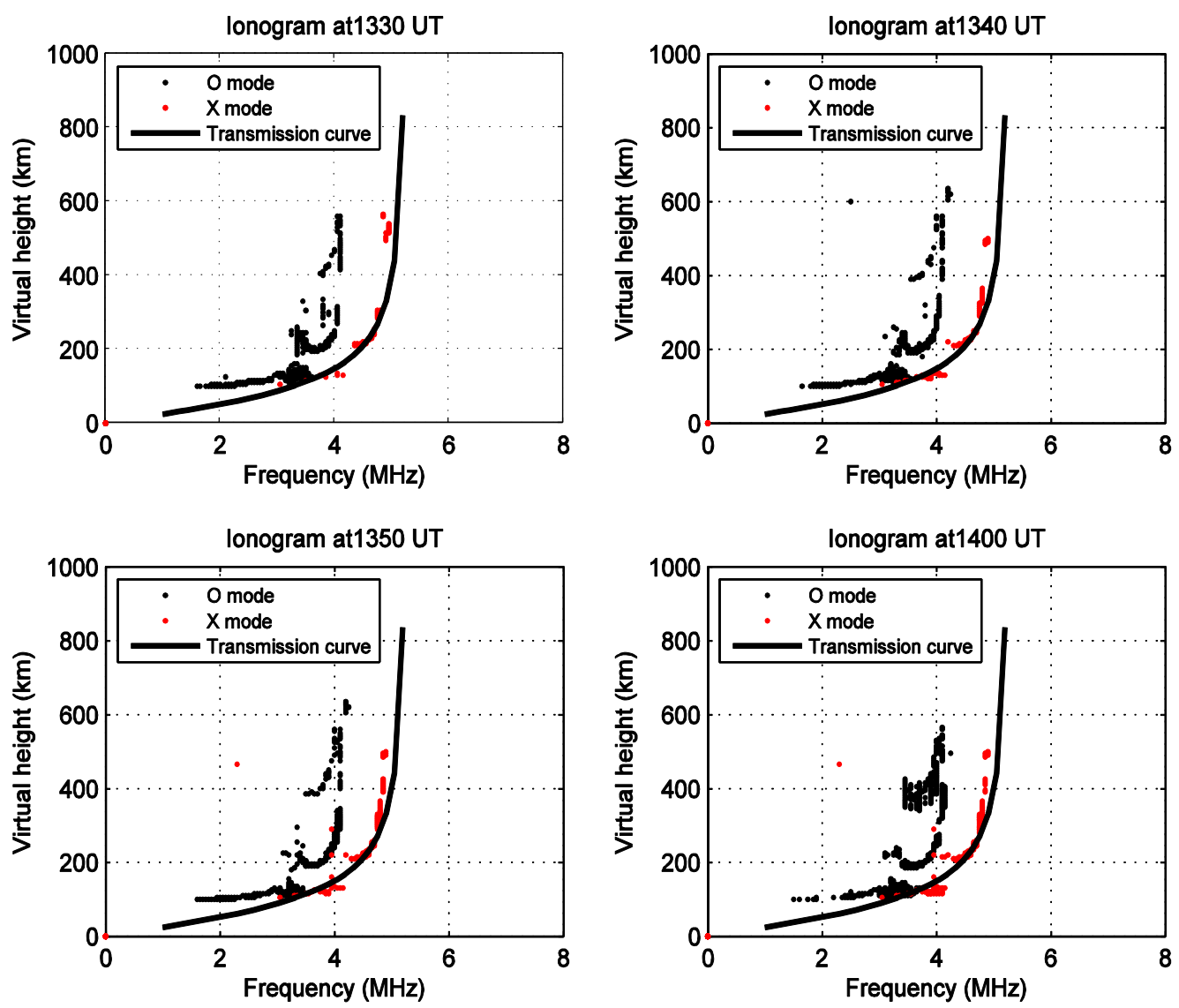

Figure 4.7 Ionograms superimposed with 5.255 MHz transmission curves (1 July 2008)

Antennas were calculated and approximately $50 \%$ of these values were found to lie below 0.9 .

In order to determine the capacity estimates for the given data Equation 2.1 was used. Capacity was calculated for each measurement using the unique $\mathrm{H}$ matrix and the the results from all measurements were then averaged. Figure 4.8 shows the improvements in the capacity expected by the configuration of two spaced vertical monopoles $40 \mathrm{~m}$ apart. We can see that at $40 \mathrm{~dB}$ the capacity of this $2 \times 2$ MIMO system is approximately $16 \mathrm{bps} / \mathrm{Hz}$ as compared to the approximately $13 \mathrm{bps} / \mathrm{Hz}$ of a SISO system. In the case of crossed wire antennas at the receiver the capacity estimates are higher as could have been expected from the correlation coefficient results. In this instance the Figure 4.9 shows that the capacity at $40 \mathrm{~dB}$ SNR to be around $19 \mathrm{bps} / \mathrm{Hz}$. The individual capacity estimates for each one minute measurement 
period for an SNR of $40 \mathrm{~dB}$ is shown in Figure 4.10. The capacity estimate for the crossed wire pair is higher than the spaced monopole array on almost all instances. Thus the spaced array occupying a large area could potentially be replaced with the collocated antennas for MIMO applications.

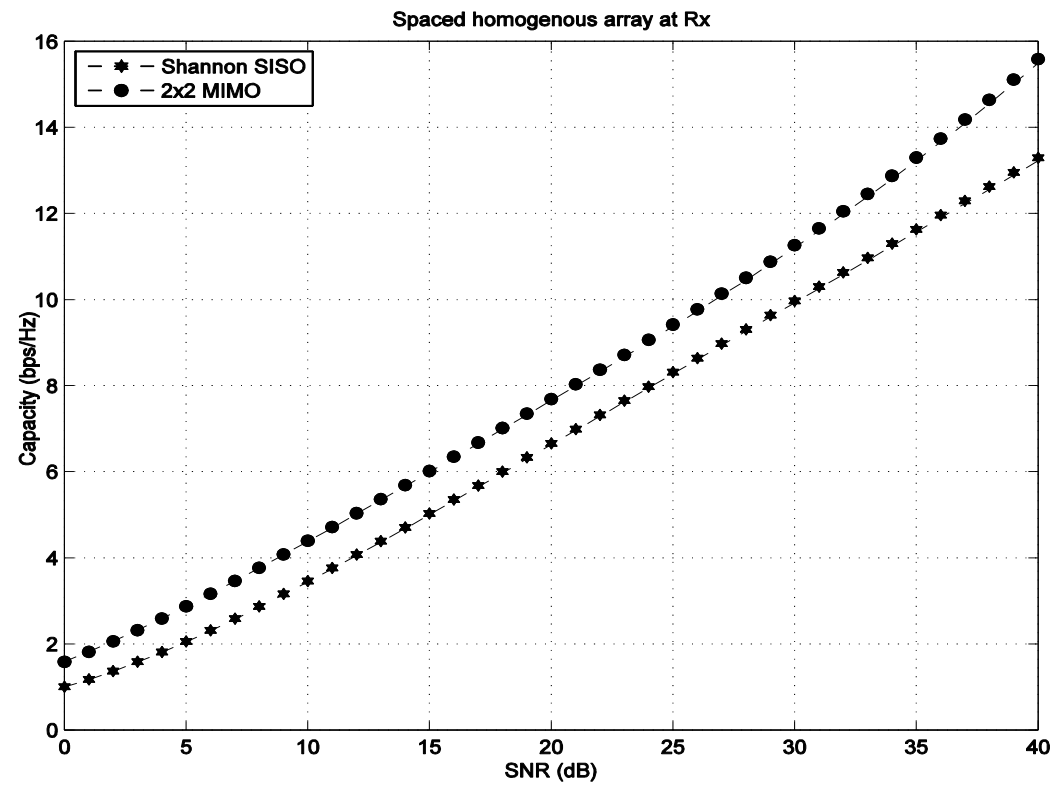

Figure 4.8 Average capacity es timates for $2 \times 2$ MIMO compared to the Shannon capacity

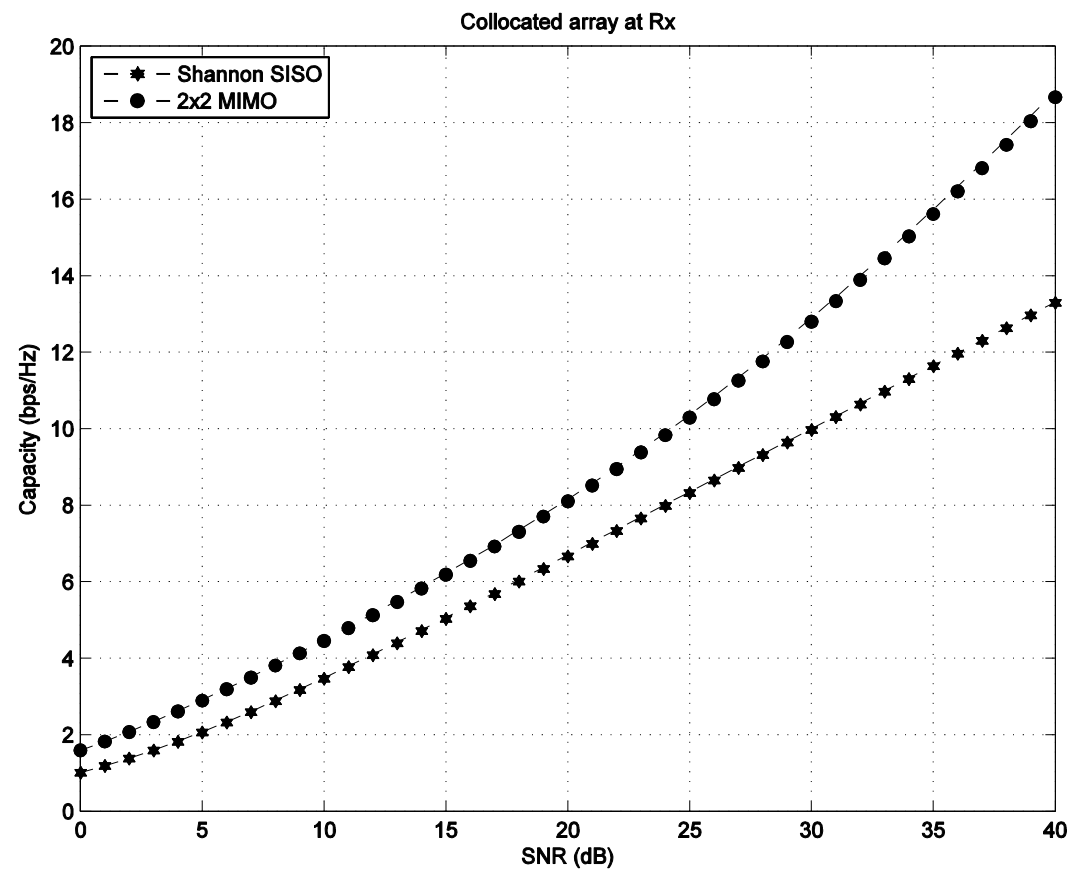

Figure 4.9 Average capacity estimates for $2 \times 2$ MIMO compared to the Shannon capacity (crossed wires at receiver) 

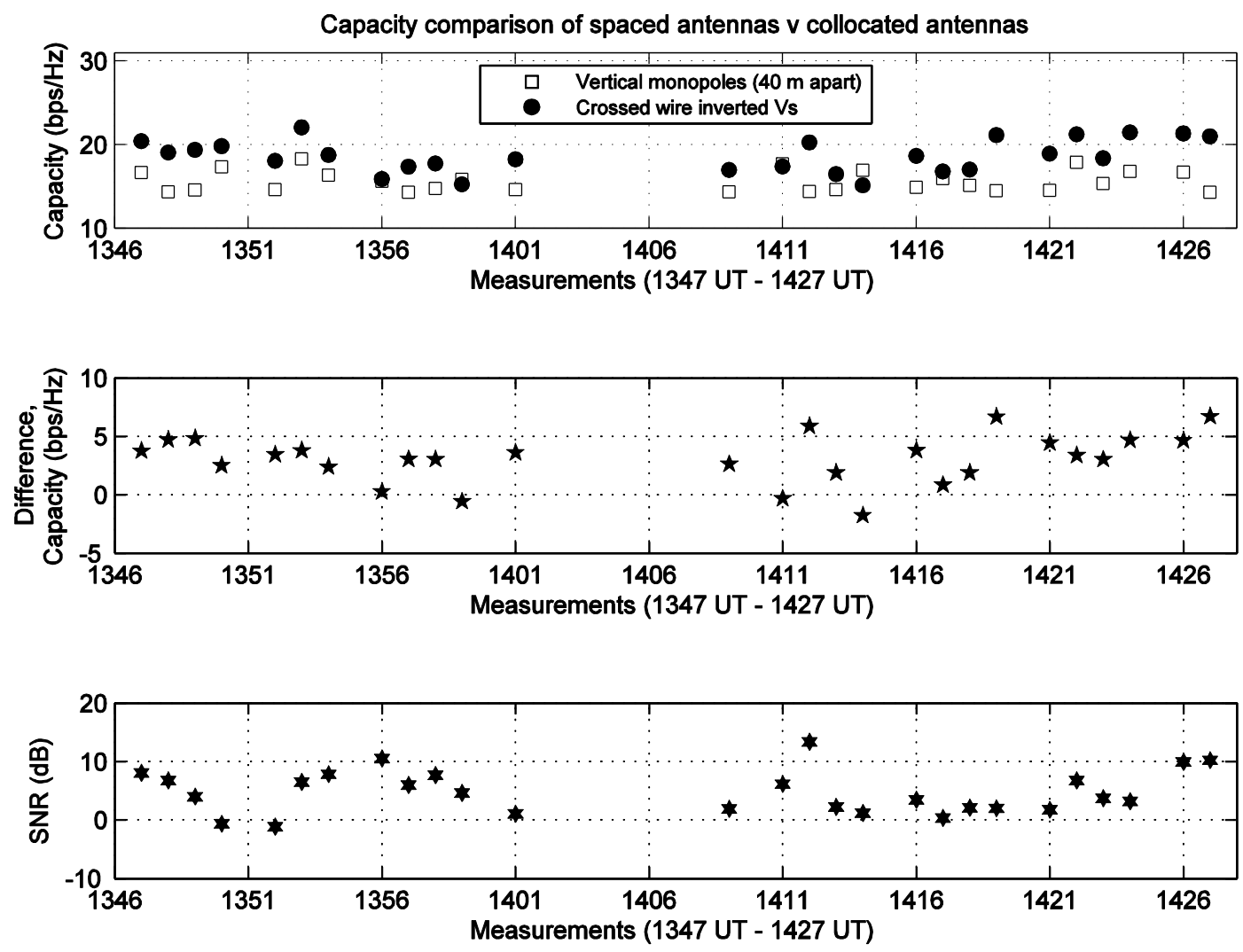

Figure 4.10 Capacity comparis on of spaced homogenous array with the crossed wire inverted $\mathrm{V}$ array at $40 \mathrm{~dB} \mathrm{SNR}$.

In the second part of this campaign data was acquired between $1451 \mathrm{UT}$ to

1521 UT. The ionospheric conditions were different with more modes present as seen in Figure 4.11. $\mathrm{X}$ mode is dominant with $\mathrm{E}$ and $\mathrm{F}$ regions reflections. It was verified using the amplitude plots verified that fading is present on the vertical monopoles and the crossed wire inverted $\mathrm{V}$ antennas. In both cases signals were found to be fading more independently than in the previous measurements.

The envelope correlation coefficients show better decorrelation in the spaced antennas as compared to the first part of this campaign where all values were above 0.9. Still the antennas are very much correlated with almost all correlation coefficients above the 0.8 value. The crossed wire inverted $\mathrm{V}$ antennas make much better use of the multipath available and are very much decorrelated. For Tx1, $73 \%$ of the correlation 
coefficient values are below 0.9 and $50 \%$ of them below 0.7 . The correlation coefficients are even lower for the Tx2.

In general the $2 \times 2$ transmit arrays are also well decorrelated as compared to the previous part especially for the vertical monopole 2 and the north-south oriented inverted $\mathrm{V}$ at the receive side.
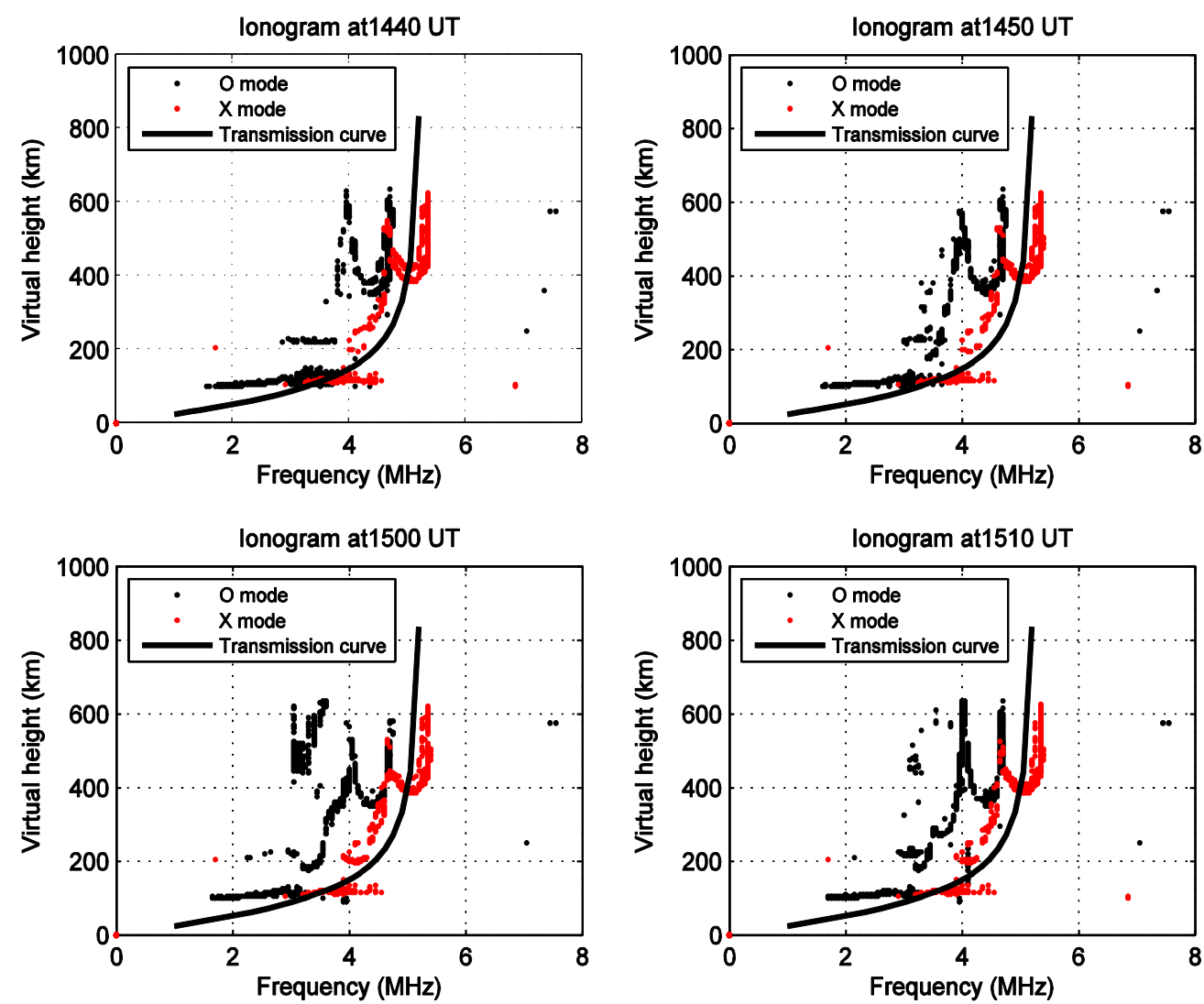

Figure 4.11 The ionograms with transmission curve overlaid, obtained from Chilton for part B of the campaign ( 1 July 2008)

The capacity curves for the collocated antennas in comparison to a Shannon SISO system capacity is shown in Figure 4.12. The same set of antennas now provides higher capacity estimates as compared to the first half of the same campaign on the same day. At $40 \mathrm{~dB}$ an improvement of about $2 \mathrm{bps} / \mathrm{Hz}$ can be seen. As the same antennas were used this increase can be attributed to the change in ionospheric conditions and availability of the greater number of modes of propagation. The 
comparison of the capacity estimates for spaced homogenous array with the collocated crossed wire array for individual measurements is done in Figure 4.13.

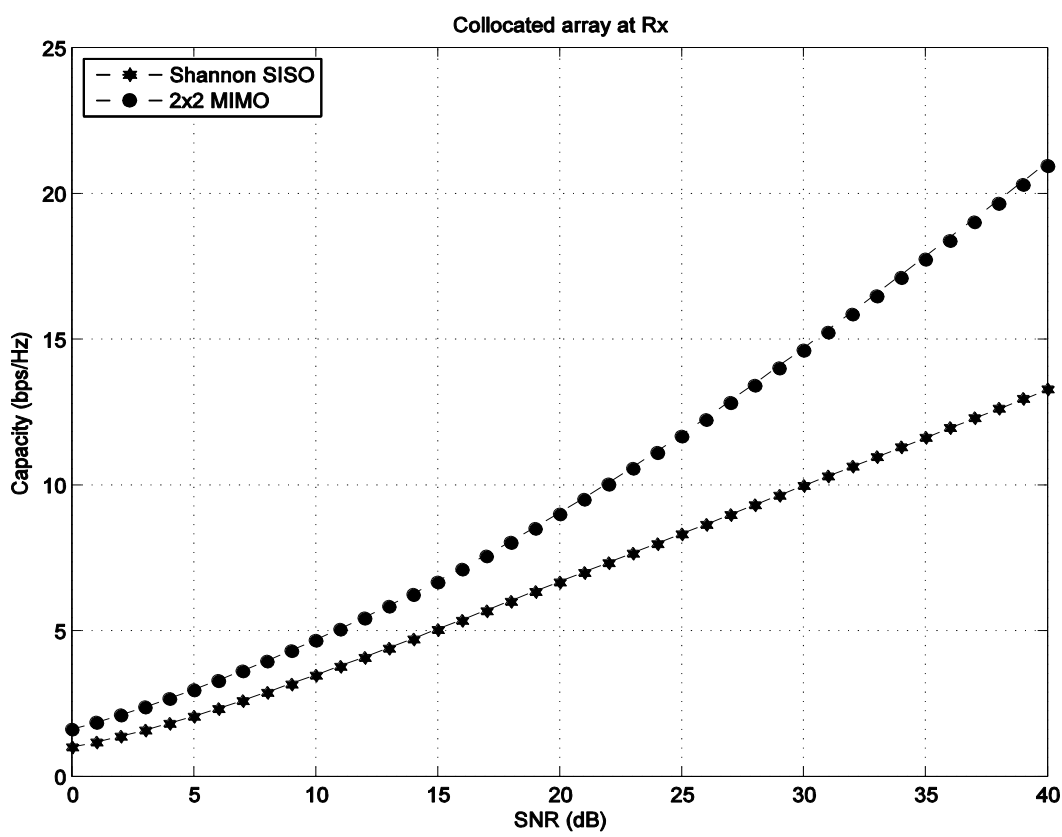

Figure 4.12 Capacity estimates for $2 \times 2$ MIMO compared to the Shannon SISO capacity (cross ed wires at receiver)
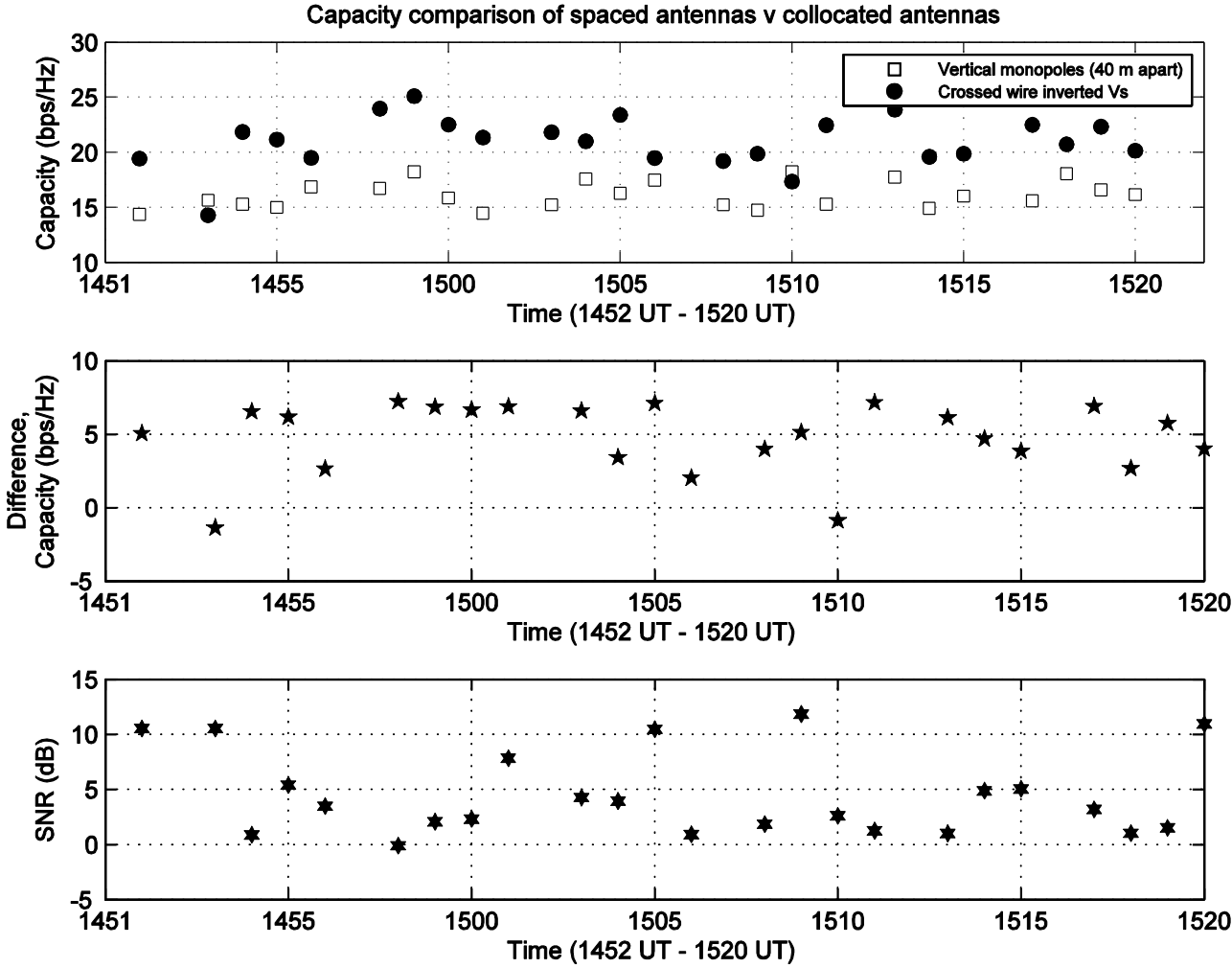

Figure 4.13 Capacity comparis on of spaced homogenous array with the crossed wire inverted $\mathrm{V}$ array at $40 \mathrm{~dB} \mathrm{SNR}$. 


\subsubsection{Comparison of spaced arrays and the collocated array in $4 \times 4$ MIMO}

This data collection campaign was carried out on 2 July 2008. In this campaign four antennas were used at the transmitter at Durham. These consisted of the north-south and east-west crossed wire inverted Vs, loop antenna and the vertical antenna. At the receiver the configuration, similar to the one shown in Figure 4.6 was used for analysis. These were the antennas of the north-south arm with the separation distance of $40 \mathrm{~m}$. The nominal frequency of $5.255 \mathrm{MHz}$ was used in this campaign with a separation of $10 \mathrm{~Hz}$ at each transmitter. Data were collected between 1005 UT and 1153 UT. The ionograms between the intervals of 1000 UT to 1050 UT are shown in Figure 4.14. The overlaid transmission curves indicate a scattering rich ionosphere at the frequency used. Both $\mathrm{O}$ and $\mathrm{X}$ mode reflections are present. Multipath propagation is prevalent in $\mathrm{E}$ and $\mathrm{F}$ regions along-with multi-hop propagation. The amplitude plots from the data show that the signal goes through regular fades on all four antennas. The spaced monopole antennas fading patterns are however similar as compared to the crossed wire inverted $\mathrm{V}$ antennas. The envelope correlation coefficients depict the decorrelation provided by the multipath environment. The vertical monopole antennas correlation coefficients are mostly in the range of $0.5-1$, however majority of the values lie between 0.5-0.9.

For the east-west inverted V antennas at transmitter the spaced homogenous array has $61 \%$ of the envelope correlation coefficient values below 0.9 and only $16.4 \%$ values below 0.7 . The collocated crossed wire inverted $\mathrm{V}$ antennas are much more de-correlated for all transmit antennas. In the case of the same east-west inverted $\mathrm{V}$ at transmit end the envelope correlation coefficient values stay below 0.9 for $82 \%$ of the times and below 0.7 for $50 \%$ of the times. Similar difference exists for other 
transmit antennas and strengthens the assumption that the spaced homogenous arrays can be replaced by appropriate collocated antennas. On the transmit side adequate decorrelations between the collocated crossed wires antennas was observed. Similar decorrelation levels were seen on the heterogeneous loop and vertical antennas at the transmission site at Durham.
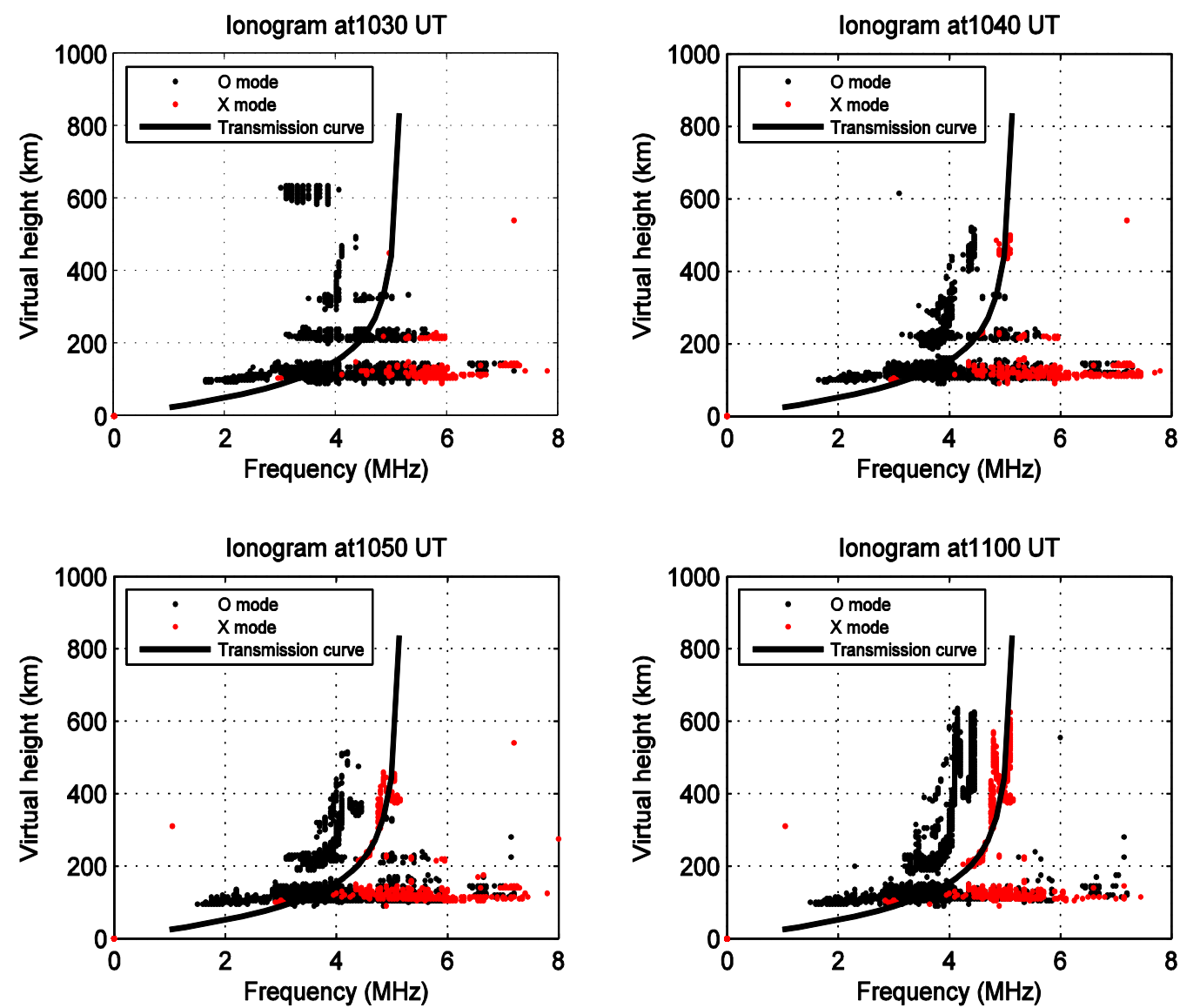

Figure 4.14 Ionograms superimposed with $5.255 \mathrm{MHz}$ trans mission curves (2 July 2008)

The capacity comparison for the two types of arrays validates the correlation coefficient results. The average capacity estimates for $2 \times 2$ spaced and collocated arrays along with Shannon SISO capacities are plotted in Figure 4.15 and Figure 4.16. The crossed wires antennas exhibit a higher degree of capacity improvement over the spaced monopole antenna arrays. The spaced array gives a capacity estimate of approximately $20 \mathrm{bps} / \mathrm{Hz}$, as compared to the $23 \mathrm{bps} / \mathrm{Hz}$ of the collocated array. The capacity comparison of the two arrays for each measurement at $40 \mathrm{~dB}$ is produced in 
the Figure 4.17, where most of the instances show a higher capacity for crossed wire antennas as compared to the spaced homogenous antennas. Also the difference in capacity between the pairs of antennas decreases with time and the capacity generally improves. The SNR also shows improvement in the measurements made in the later part.

These experimental results clearly show that the spaced homogenous array can be replaced by the large collocated antenna arrays without any compromise on the ability to decorrelate. In fact even the separation of greater than $\lambda / 2$ (in case of $40 \mathrm{~m}$ separation) is insufficient to provide a higher level of decorrelation. To provide similar levels of decorrelation the distances have to be further increased to greater than $\lambda$ which would require a separation distance of more than $60 \mathrm{~m}$ for the $2 \times 2 \mathrm{MIMO}$ array.

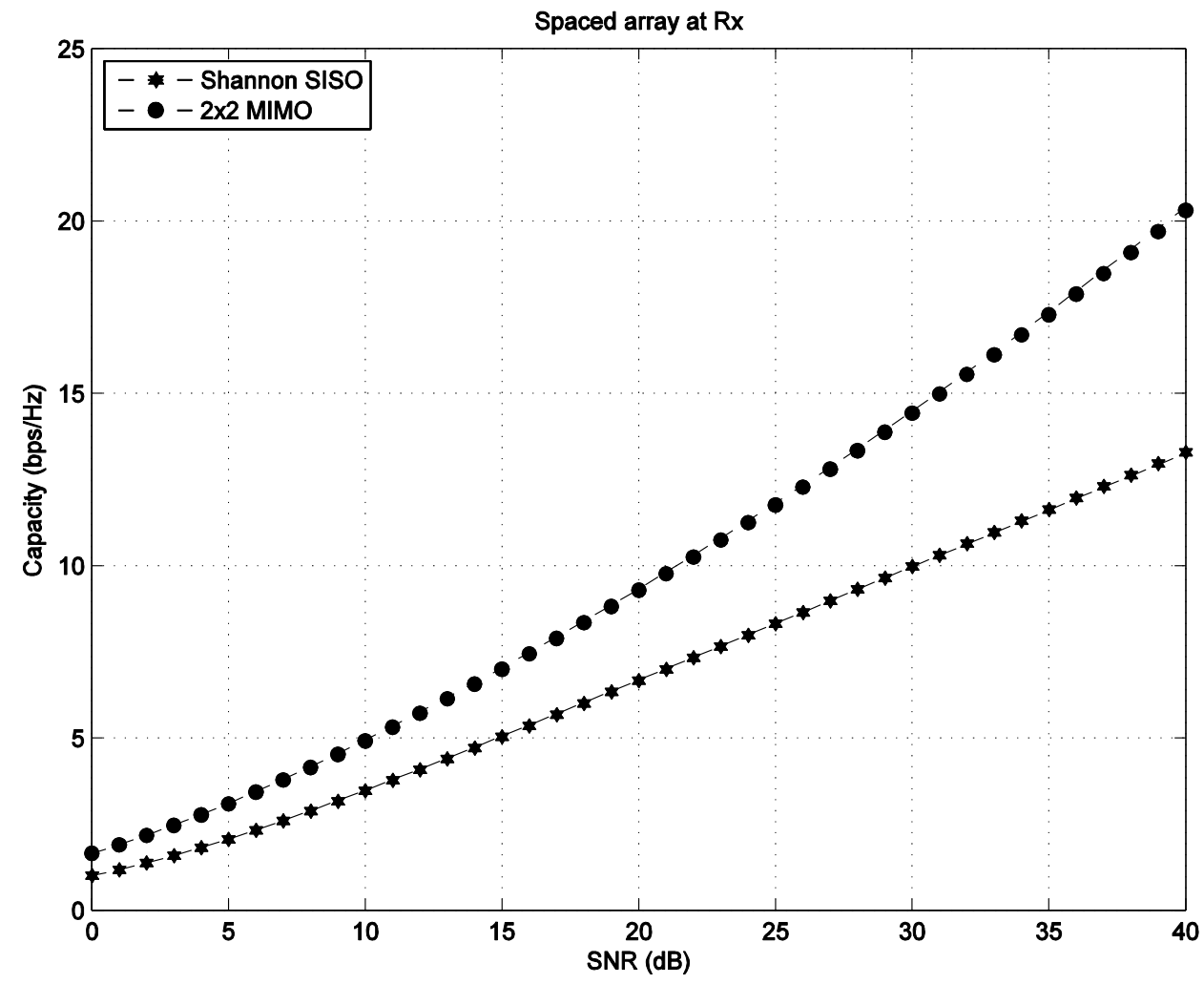

Figure 4.15 Aver age capacity estimates for $2 \times 2$ MIMO compared to the Shannon capacity (S paced monopoles at receiver 


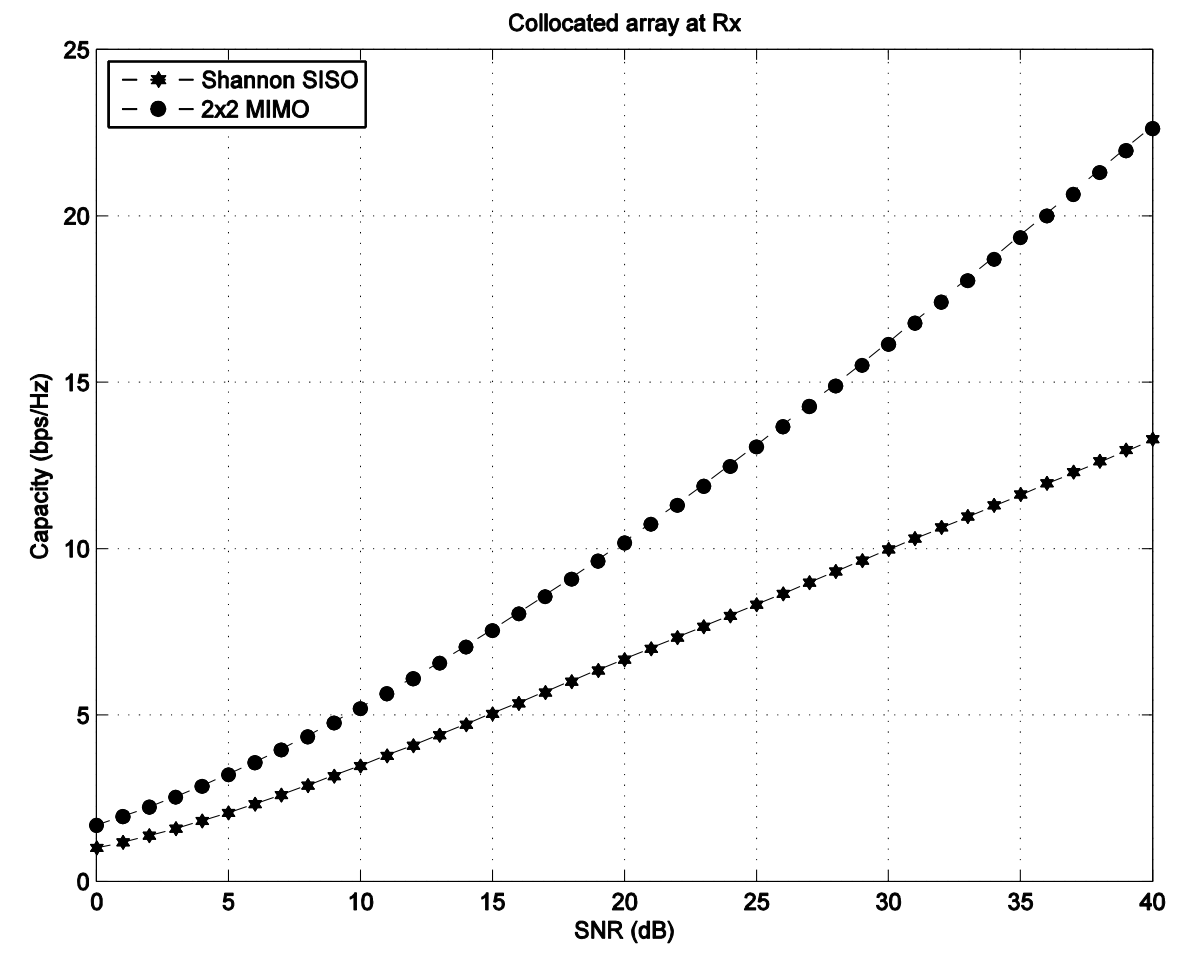

Figure 4.16 Aver age capacity estimates for $2 \times 2$ MIMO compared to the Shannon capacity (Coll ocated crosses wires at receiver)
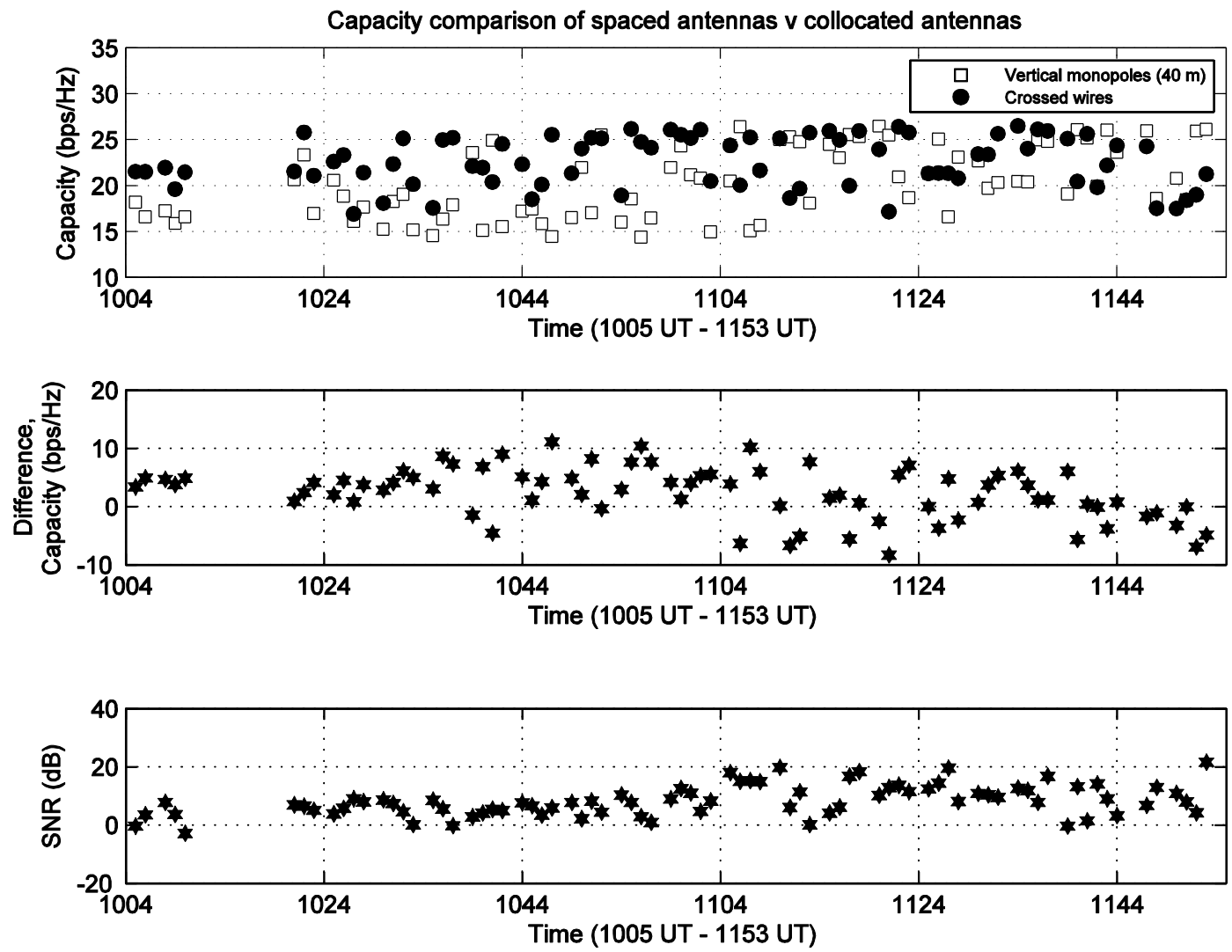

Figure 4.17 Capacity comparis on of spaced homoge nous array with the crossed wire inverted $V$ array at $40 \mathrm{~dB} S \mathrm{SR}$. 


\subsubsection{Comparison of crossed wire array with smaller loop array and monopole array in a $3 \times 8$ MIMO configuration}

Data for this campaign was collected on 29 July 2008. In this campaign the transmit side consisted of crossed wire inverted Vs, loop and vertical antenna (not used in the analysis). At the receiver end all 8 channels were utilized. Three different configurations of collocated antennas were used. The first two channels were connected to the crossed wire inverted $\mathrm{V}$ antennas. The second array was composed of collocated active monopoles oriented north-south, east-west and vertical and the third array consisted of north-south, east-west and horizontal oriented active loop antennas. The frequency of operation was 5.255 MHz. Again the frequency offset of $10 \mathrm{~Hz}$ was used at each transmit antennas for identification of the transmitting antennas at the receiver. Data was collected between 1151 UT to 1306 UT.

The ionograms between the intervals of 1200 UT to 1250 UT are presented in
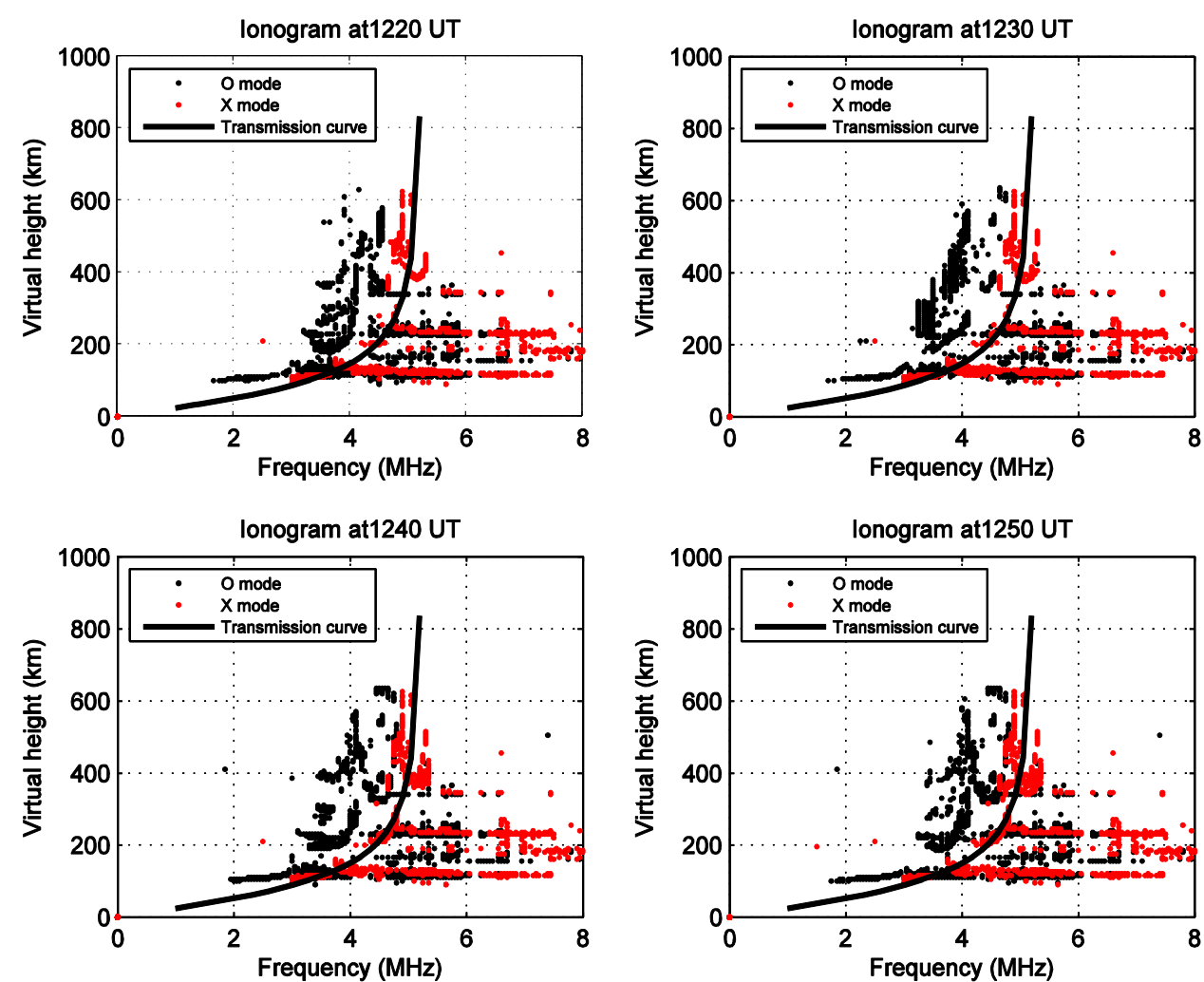

Figure 4.18 Ionograms superimposed with 5.255 MHz transmission curves (29 July 2008) 
Figure 4.18. Both $\mathrm{O}$ and $\mathrm{X}$ mode reflections are present in $\mathrm{E}$ and $\mathrm{F}$ regions. Multi-hop propagation is also witnessed however $\mathrm{O}$ mode is dominant at the frequency used. From the amplitude plots it was observed that the fading on different receivers is quite independent and good decorrelation results could be expected.

The envelope correlation coefficients for the crossed wire inverted Vs were compa-red with the collocated active monopoles. The monopoles show very high correlations with majority of the values in the 0.9-1 range. Similar results were obtained with the other two monopole pairs. Thus the monopoles are not able to decorrelate and utilize the multipath available. This can be due to the fact that the monopoles would be receiving the same wave front without any difference in the phase and there is no polarization or pattern diversity offered by such antennas. In the case of crossed wire antennas $91 \%$ of the correlation coefficient values were found to be below 0.9 value while $54 \%$ were below 0.7 . The loop pairs provide decorrelation comparable to the crossed wires. The north-south and east-west pair have $83 \%$ correlation values below 0.9 and $70 \%$ below 0.7 . North-south and east-west have $89 \%$ and $30 \%$ values below 0.9 and 0.7 respectively. The East-west and the horizontal pair decorrelations are under 0.9 and 0.7 for $90 \%$ and $73 \%$ of times. This result is promising because the loop array occupies an area of $1 \mathrm{~m}^{2}$ which is small enough to be accommodated in almost all applications of HF communications. The crossed wires on the other hand take a space of $28 \mathrm{~m}^{2}$ and the spaced antennas have to be placed at-least half a wavelength apart to provide decorrelation. The transmit crossed wire inverted V antennas are also very well de-correlated. In terms of antenna heterogeneity the north-south inverted $\mathrm{V}$ and vertical antenna perform much better than the east-west inverted $\mathrm{V}$ and the vertical antenna. Thus antenna heterogeneity in 
itself cannot guarantee decorrelation and not all heterogeneous antennas can be effectively be used to increase data rate when utilized in MIMO.

The capacity estimates for this campaign were computed for the three collocated arrays i.e. crossed wires, collocated monopoles and collocated loop antennas (Figure 4.19, Figure 4.20, Figure 4.21). The results authenticate the deductions from the envelope correlations for these antennas. For the $2 \times 2$ MIMO the collocated north-south and east-west loops at the receiver along with the crossed wires at transmit end show similar performance to the crossed wire antennas at both the transmit and receive ends of the link.

The capacity estimates for the collocated north-south and east-west monopoles at the receiver with the crossed wires at the transmitter show a performance degradation of about $3 \mathrm{bps} / \mathrm{Hz}$. The $3 \times 3 \mathrm{MIMO}$ performance of the collocated loops is also superior to the collocated monopoles by a margin of around $4 \mathrm{bps} / \mathrm{Hz}$.

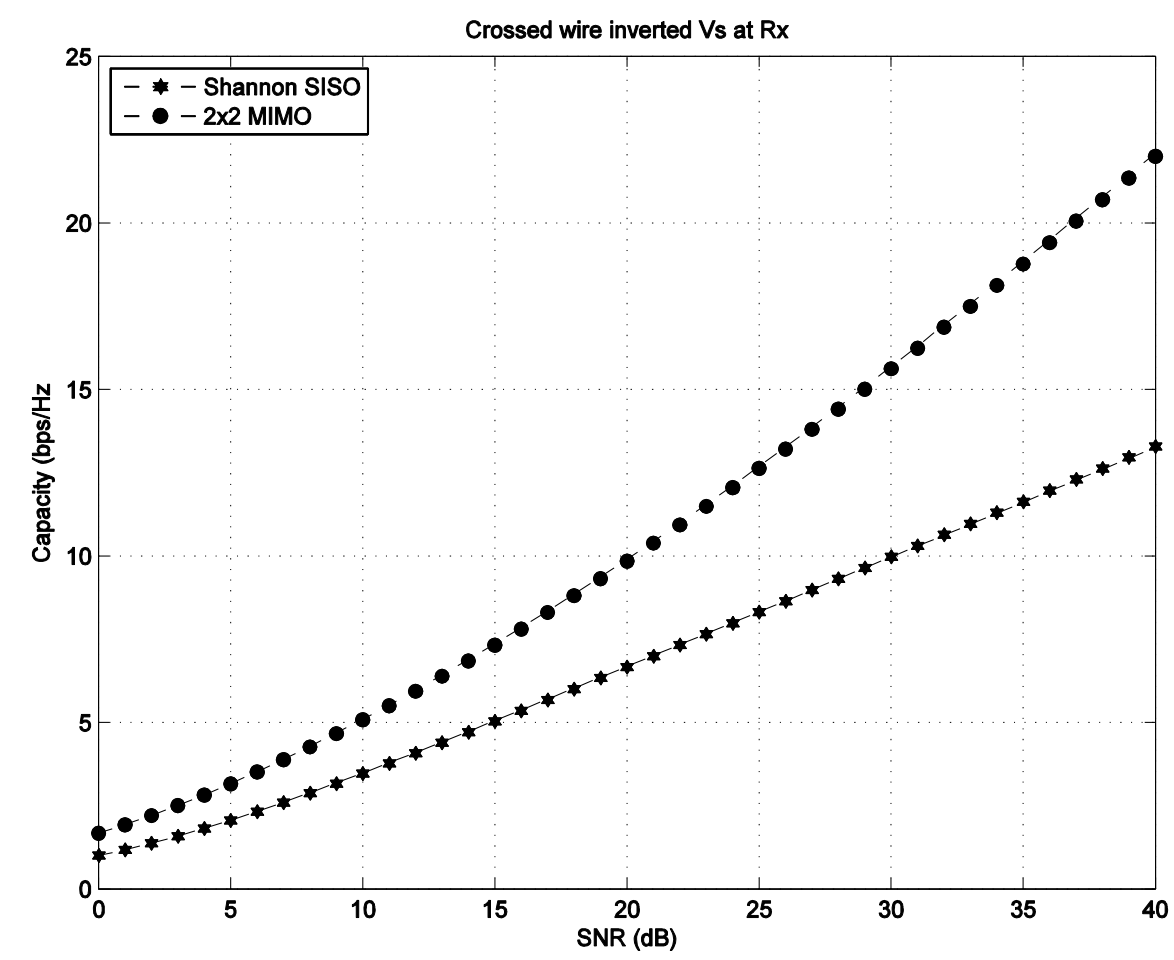

Figure 4.19 Capacity es ti mates for $2 \times 2$ MIMO compared to the Shannon SISO capacity (crossed wires at receiver) 


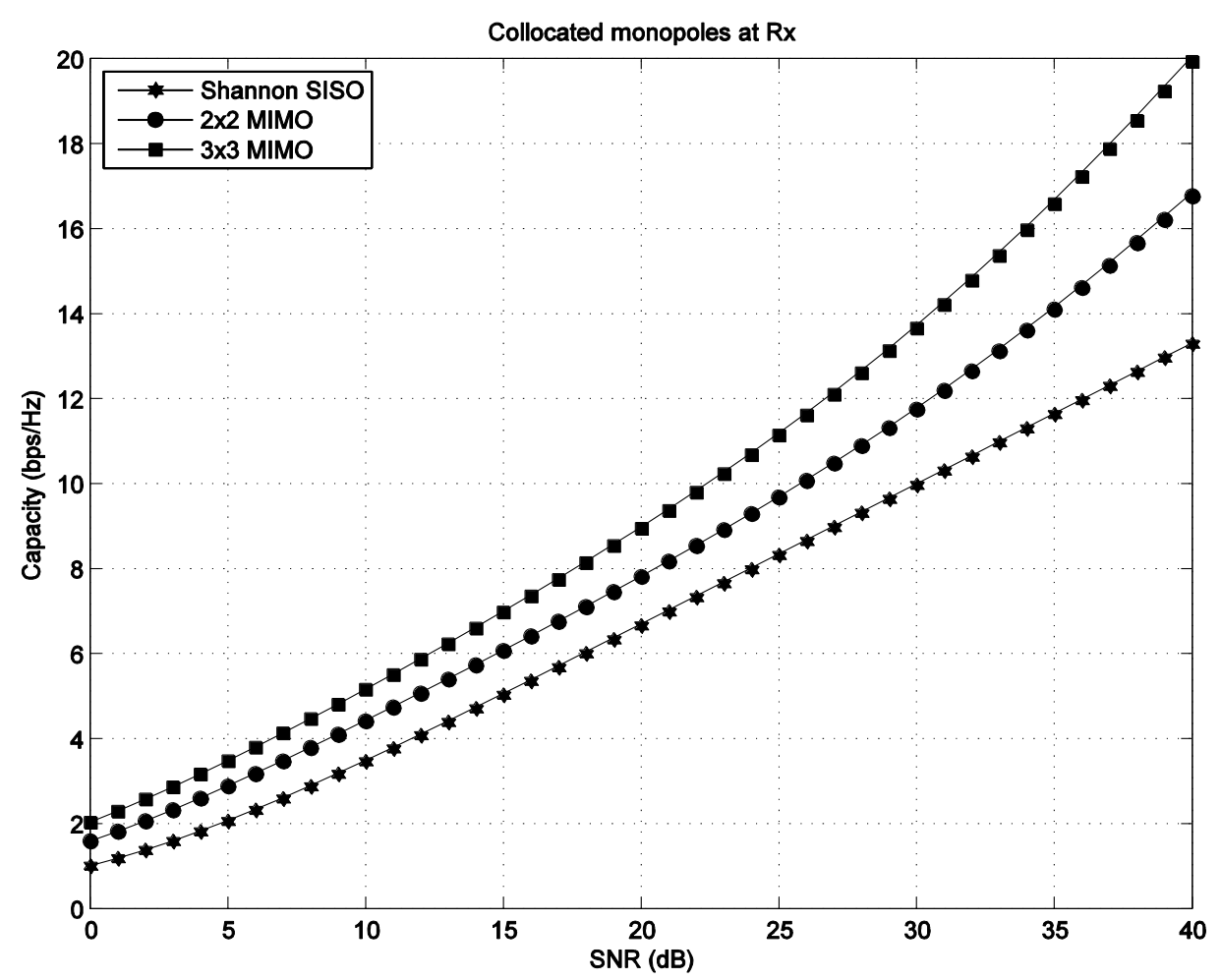

Figure 4.20 Capacity estimates for $2 \times 2$ and $3 \times 3$ MIMO compared to the Shannon S ISO capacity (collocated monopoles at receiver)

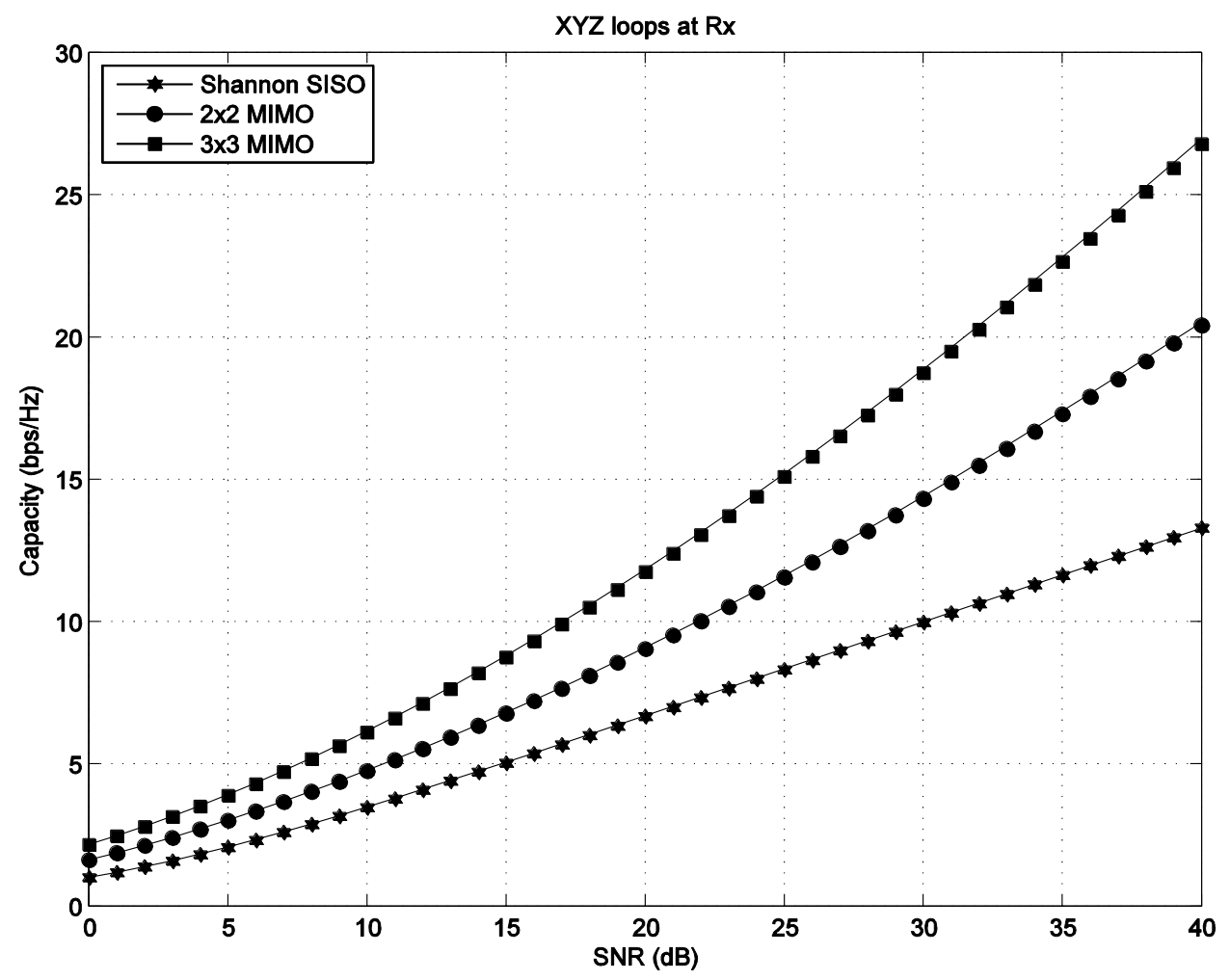

Figure 4.21 Capacity es timates for $2 \times 2$ and $3 \times 3$ MIMO compared to the Shannon S ISO capacity (XYZ loops at receiver) 


\subsubsection{Comparison of large collocated array and small collocated active array in $4 \times 8$ MIMO configuration}

In this campaign carried out on 7 August 2008, 4x8 MIMO was employed. The receiving antennas consisted of a spaced array and two different collocated arrays. The spaced array was made of the 3 antennas comprising the north-south arm of the Lshaped array and the results were presented in section 4.1.2. Two crossed wire inverted V antennas made the second array and the third array consisted of the XYZ loop array. Such an arrangement would provide a comparison between the performance of a spaced homogenous array, large collocated array and small collocated array under similar conditions. The transmit antennas also included a crossed wire inverted $\mathrm{V}$ array similar to the one at the receiver end. In addition the spaced loop and vertical antenna were also used.

The nominal frequency of $5.255 \mathrm{MHz}$ was used with a separation of $10 \mathrm{~Hz}$ at each transmitter (Tx1-5.255010, Tx2-5.255020, Tx3-5.255030 and Tx4-5.255040). The representative ionograms for this campaign are shown in Figure 4.5. The data were collected between 1158 UT to 1358 UT.

The correlation coefficients for the crossed wire antennas stay below the value of 0.9 in $64 \%$ and below 0.7 for $14 \%$ of the measurements periods. The north-south and horizontal antenna pair in the XYZ array is less de-correlated with only $53 \%$ values below 0.9 and $7 \%$ below 0.7 . The north-south and east-west pair and the eastwest and horizontal pair however perform better than the cross ed wires. The northsouth and east-west antennas correlation coefficients measurements stay below 0.9 and 0.7 for $91 \%$ and $58 \%$ of the times. In case of east-west and horizontal pair it is $79 \%$ and $29 \%$ for values below 0.9 and 0.7 . Thus on the whole the XYZ loops are able to decorrelate the multipath signals and can be used to replace the larger crossed wire 
antennas. On the transmit side the crossed wires and the spaced heterogeneous pair of loop and vertical antennas show similar correlation coefficients results.

The capacity estimate at $40 \mathrm{~dB}$ SNR is presented in Figure 4.22 along with the capacity difference and the measured SNR. The XYZ loop and the crossed wire antennas provide similar levels of capacity on average with the former pair of antennas leading the later on more occasions. This further strengthens the argument for using compact collocated antenna arrays in HF-MIMO applications. The SNR in the bottom part of Figure 4.22 shows fading underwent by the signal.
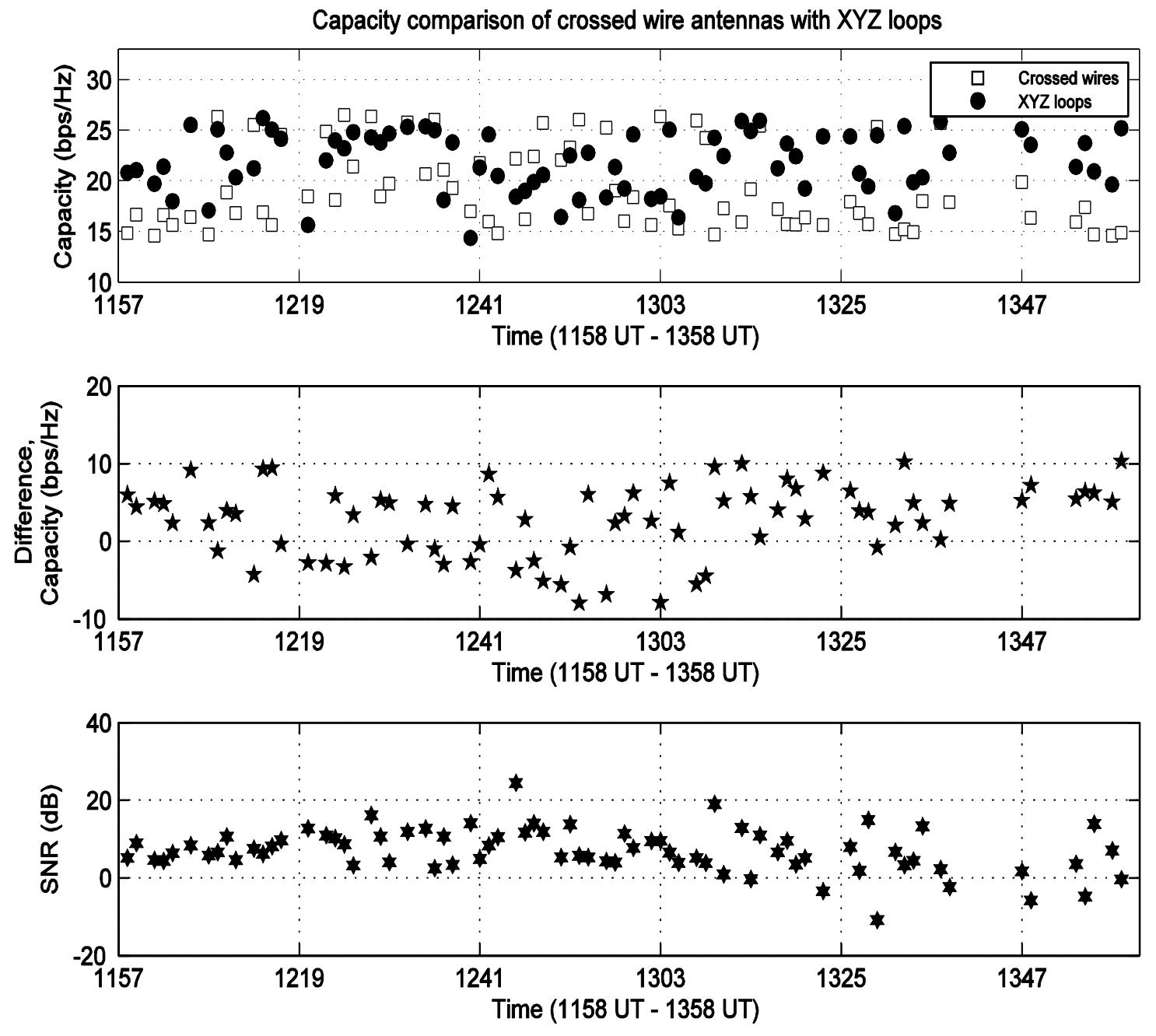

Figure 4.22 Capacity comparis on of the crossed wire inverted $V$ array with the $X Y Z$ loops (N-S \& E-W) at $40 \mathrm{~dB}$ SNR 


\subsubsection{Comparison of large collocated array (crossed wires) and small collocated array (active dipoles+monopole) in $2 \times 8$ MIMO configuration}

In this campaign carried out on 26 March 2009 a 2x8 MIMO system was set up. The receiver end consisted of the crossed wire north-south and east-west inverted $\mathrm{V}$ antennas on channel 1 and 2. A new small antenna array was used at channel 3, 4 and 5. This was made up of two horizontally polarized dipole antennas oriented northsouth and east-west, and a vertical monopole with the base at the middle of both the other antennas. Collocated north-south, east-west and horizontal loop antennas were used at channels 6,7 and 8 respectively. On the transmit side crossed wire north-south and east-west antennas were used at channel 1 and 2 . The nominal frequency of 4.455 MHz was used with a separation of $10 \mathrm{~Hz}$ at each transmitter. Data was collected between 1234 UT to 1455 UT. Four representative ionograms during this period from Chilton ionosonde with transmission curves at the nominal frequency of
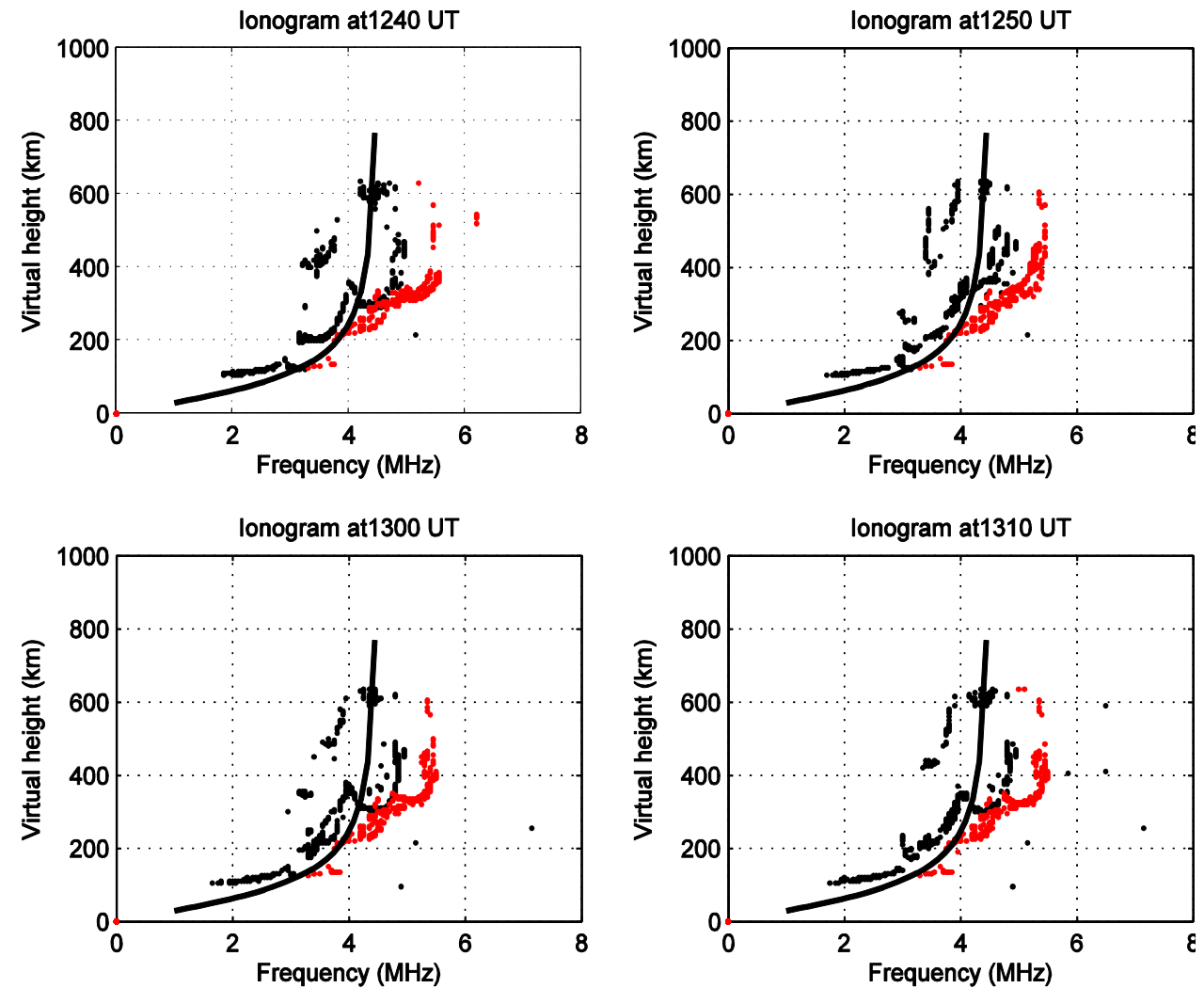

Figure 4.23 Ionogram's superimposed with 4.455 MHz transmission curves (26 March 2009) 
4.4455 MHz are shown in Figure 4.23. The ionograms depict the presence of multipath and multimodal propagation.

For the crossed wire pair the correlation coefficients stays below 0.9 for $56 \%$ of the time and below 0.7 for $16 \%$ of the time. The much smaller dipole pair performs better under the prevailing ionospheric conditions. The correlation coefficient staying below 0.9 for $62.5 \%$ of the time and below 0.7 for $20 \%$ of the time. The other two pairs comprising the vertical monopole also show decorrelation with the east-west dipole and vertical monopole pair performing better than the north-south and vertical monopole pair. The correlation coefficient for loops antennas are shown in Figure 4.24 and Figure 4.25. The north-south and east-west pair provide a decorrelation of less than 0.9 for $77 \%$ of the times and less than 0.7 for $37 \%$ of the values.
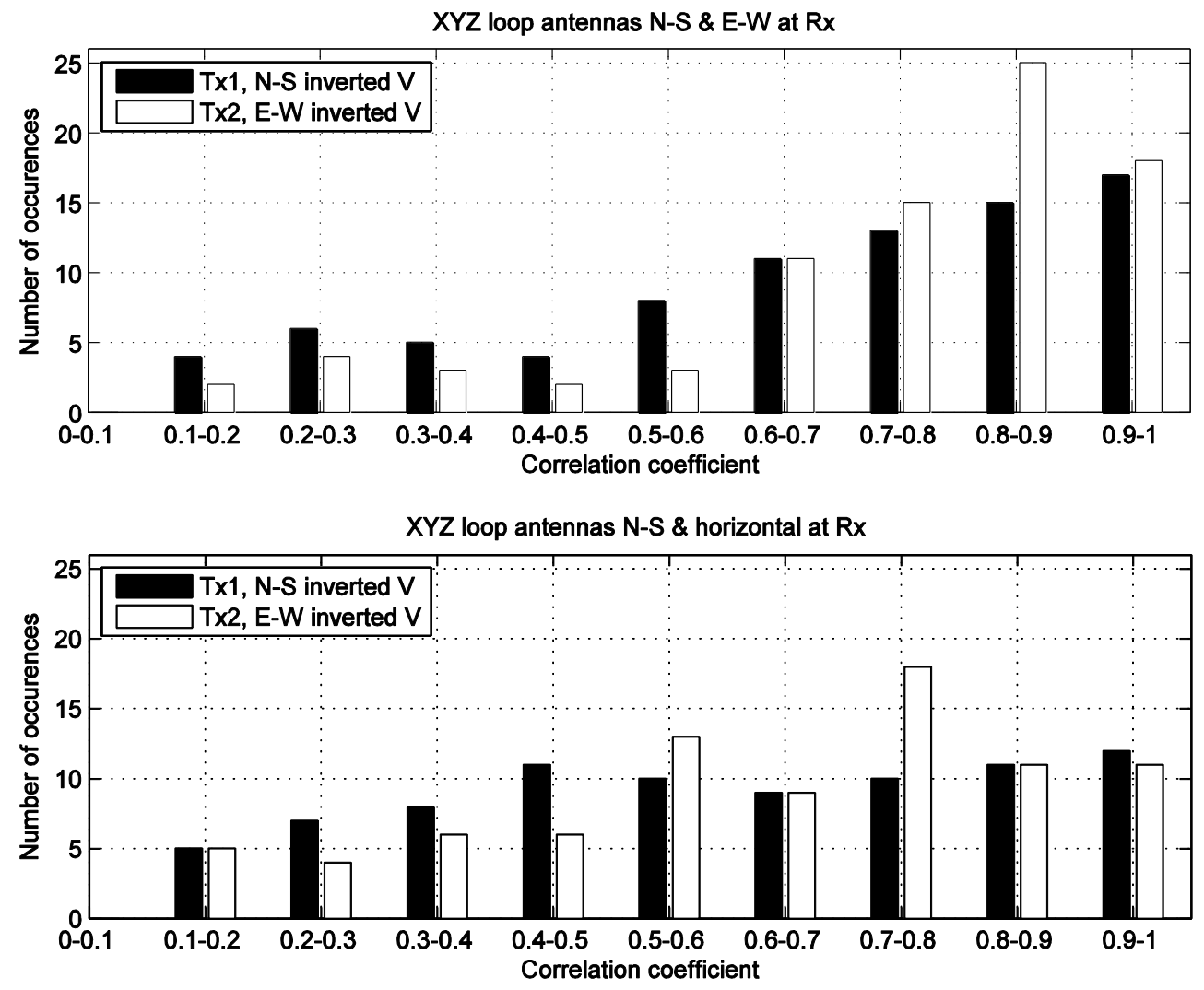

Figure 4.24 Envelope correlation coefficient histograms for crossed wire inver ted Vs (top) and coll ocated loops (north-s outh and east-west) 
$\mathrm{XYZ}$ loop array antennas E-W \& Horizontal at Rx

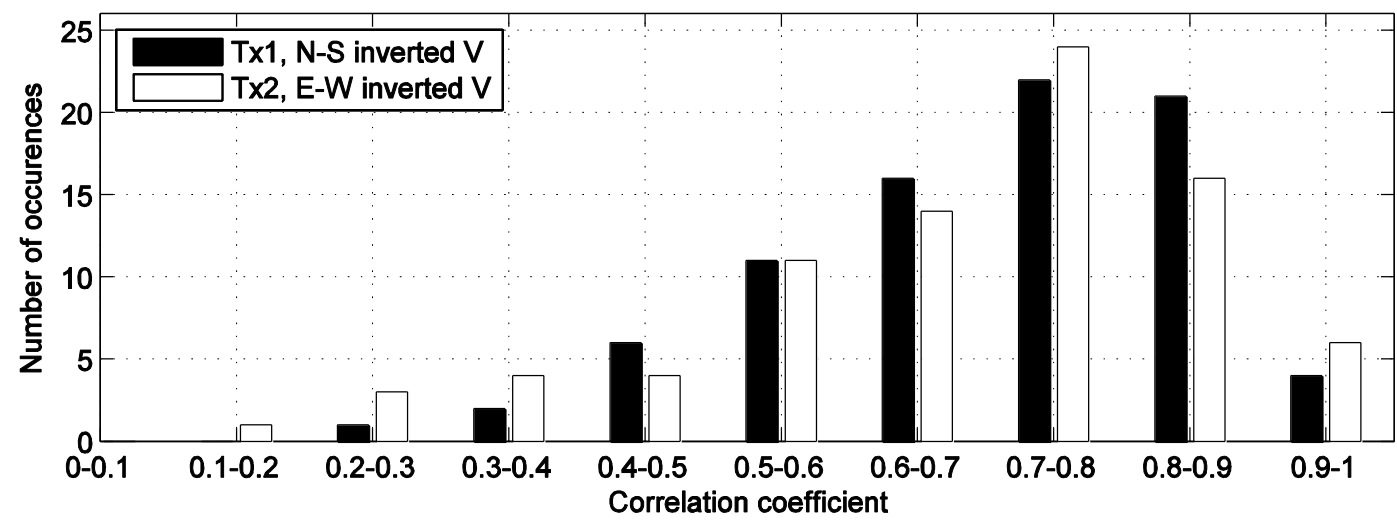

Figure 4.25 Envelope correlation coefficient histograms for crossed XYZ loops (east-west and horizontal)

Again it has been shown in this campaign that different arrangements of small collocated antennas could be effectively used for HF-MIMO applications. In this case dipole array was successfully employed giving adequate levels of decorrelation. The capacity estimates for crossed wire inverted $\mathrm{V}$ pair are compared with those from the collocated dipole antennas in Figure 4.26. It can be seen that the dipoles provide comparable levels of capacity estimates.
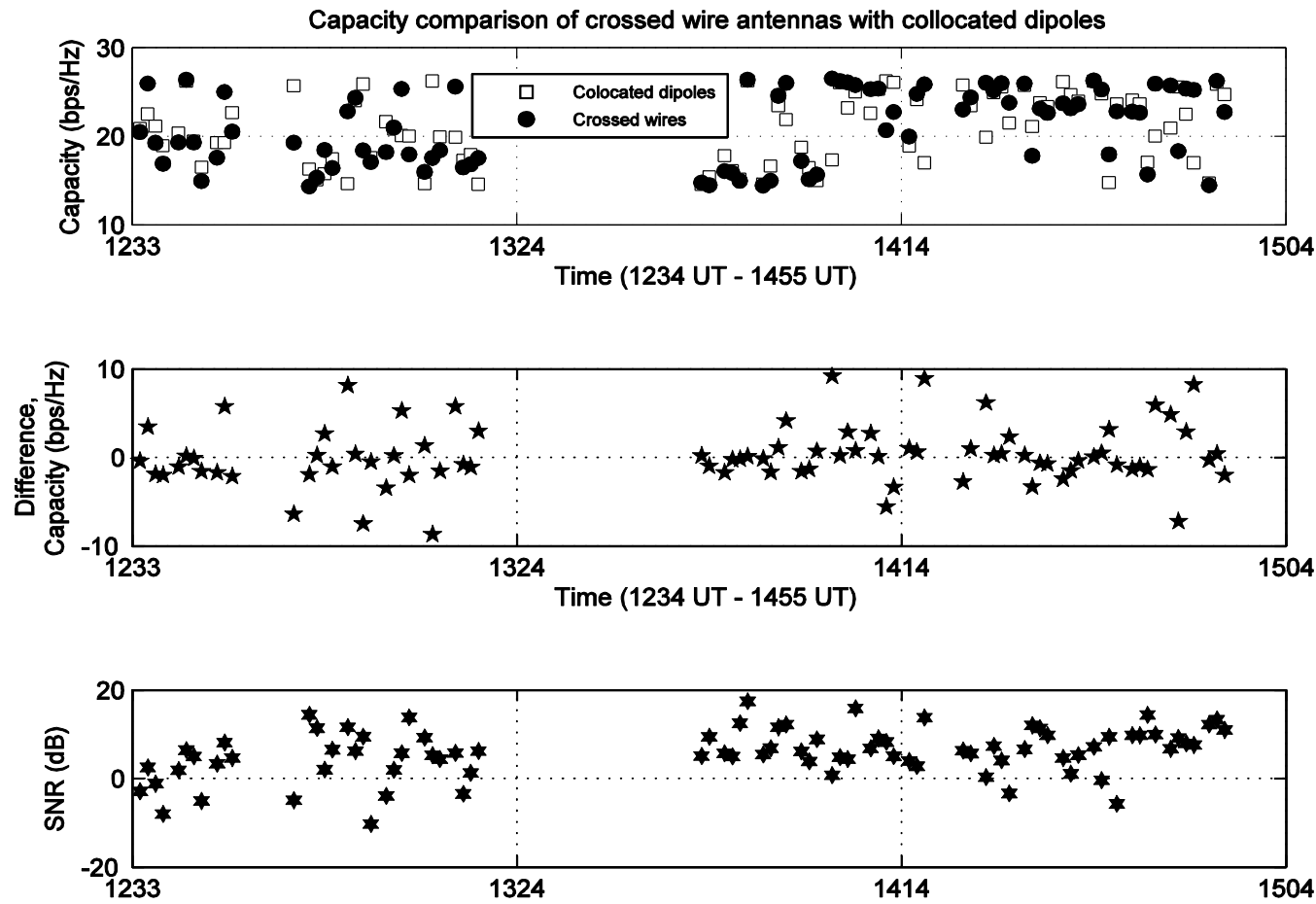

Figure 4.26 Capacity comparison of crossed inverted $V$ array with collocated dipoles at $40 \mathrm{~dB}$ SNR. 


\subsubsection{Comparison of large collocated array and small collocated Giselle array in $2 \times 8 \mathrm{MIMO}$ configuration}

In this campaign carried out on 19 June 2009 the receiver end cons isted of the same crossed wire N-S and E-W inverted V antennas on channel 1 and 2. Collocated northsouth, east-west and horizontal loop antennas were used at channels 6,7 and 8 respectively. Giselle loop array as described in chapter 3 was used in this experiment with individual antennas connected to channe1 3, 4 and 5. On the transmit side crossed wire north-south and east-west antennas were used at channel 1 and 2 . The nominal frequency of $5.255 \mathrm{MHz}$ was used with a separation of $10 \mathrm{~Hz}$ at each transmitter. The data was collected between 1135 UT to 1220 UT. Four representative ionograms during this period from Chilton ionosonde with transmission curves at the nominal frequency of 5.255 MHz are shown in Figure 4.27. The ionogram's are rarefied and limited number of modes are present.
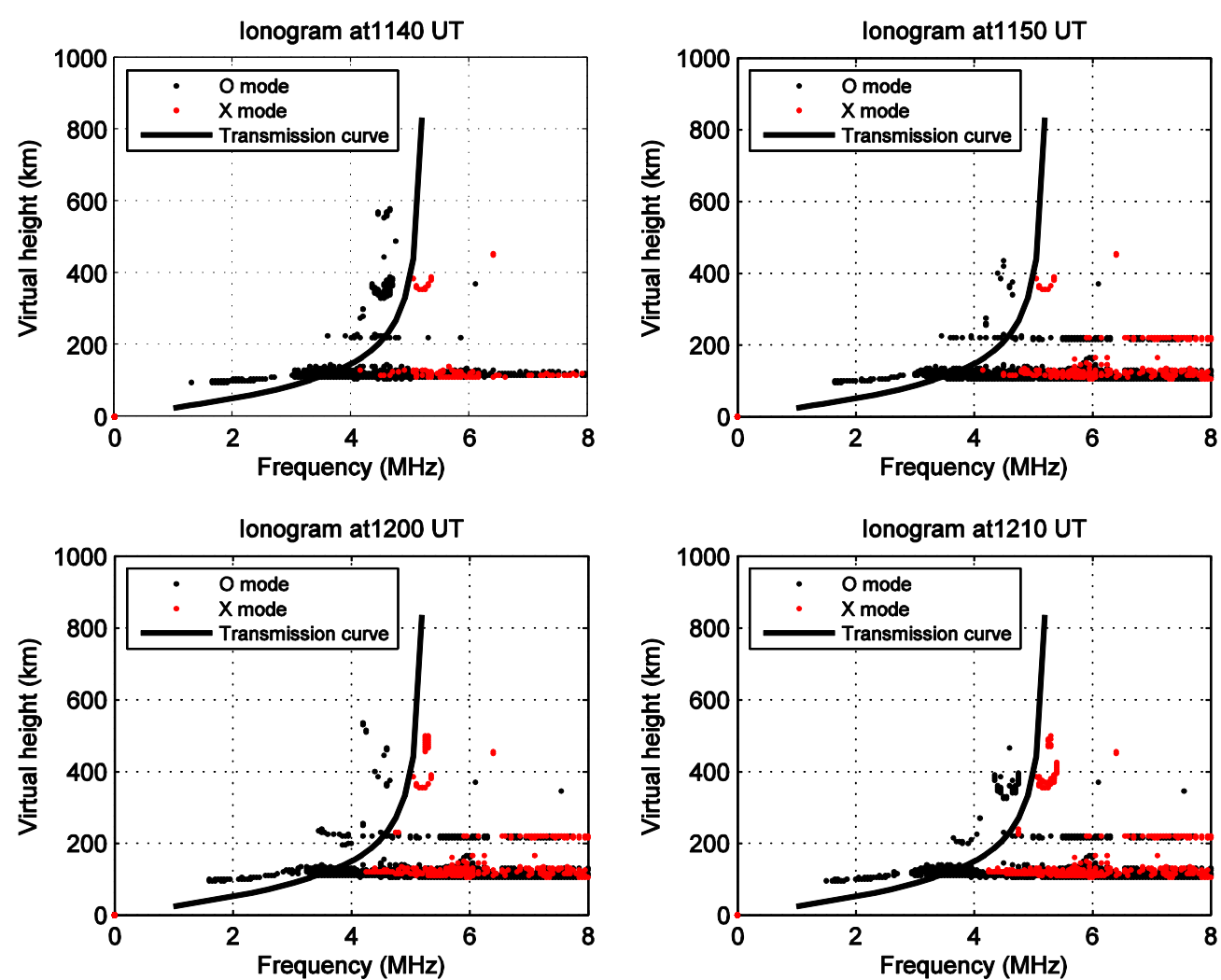

Figure 4.27 Ioogram's superimposed with 5.255 MHz transmission curves (19 June 2009) 
The comparison of envelope correlation coefficients between crossed wire inverted $\mathrm{V}$ antennas and Giselle loops 1 and 2 are shown in Figure 4.28. Both pairs show decorrelations of similar levels. The correlation coefficients for crossed wire antennas stay above 0.9 for $74 \%$ of the values while if we consider the decorrelation threshold of 0.7 , only $17 \%$ of the results are below that. For the Gissele loops $71 \%$ of the correlation coefficient values lie below 0.9 and $23 \%$ below 0.7 . The Giselle antennas 1 and 3 are very much correlated while the Giselle antennas 2 and 3 show a high level of decorrelation as depicted in Figure 4.30. The difference in the levels of decorrelation between pairs of antennas in an array could be due to the relative phase response to the incoming multipath components. The multipath components arriving at different elevation angles will excite the antennas in different ways and thus different decorrelation results for pairs of antennas in an array will be obtained. However a general conclusion can be derived about the performance of an antenna array in terms
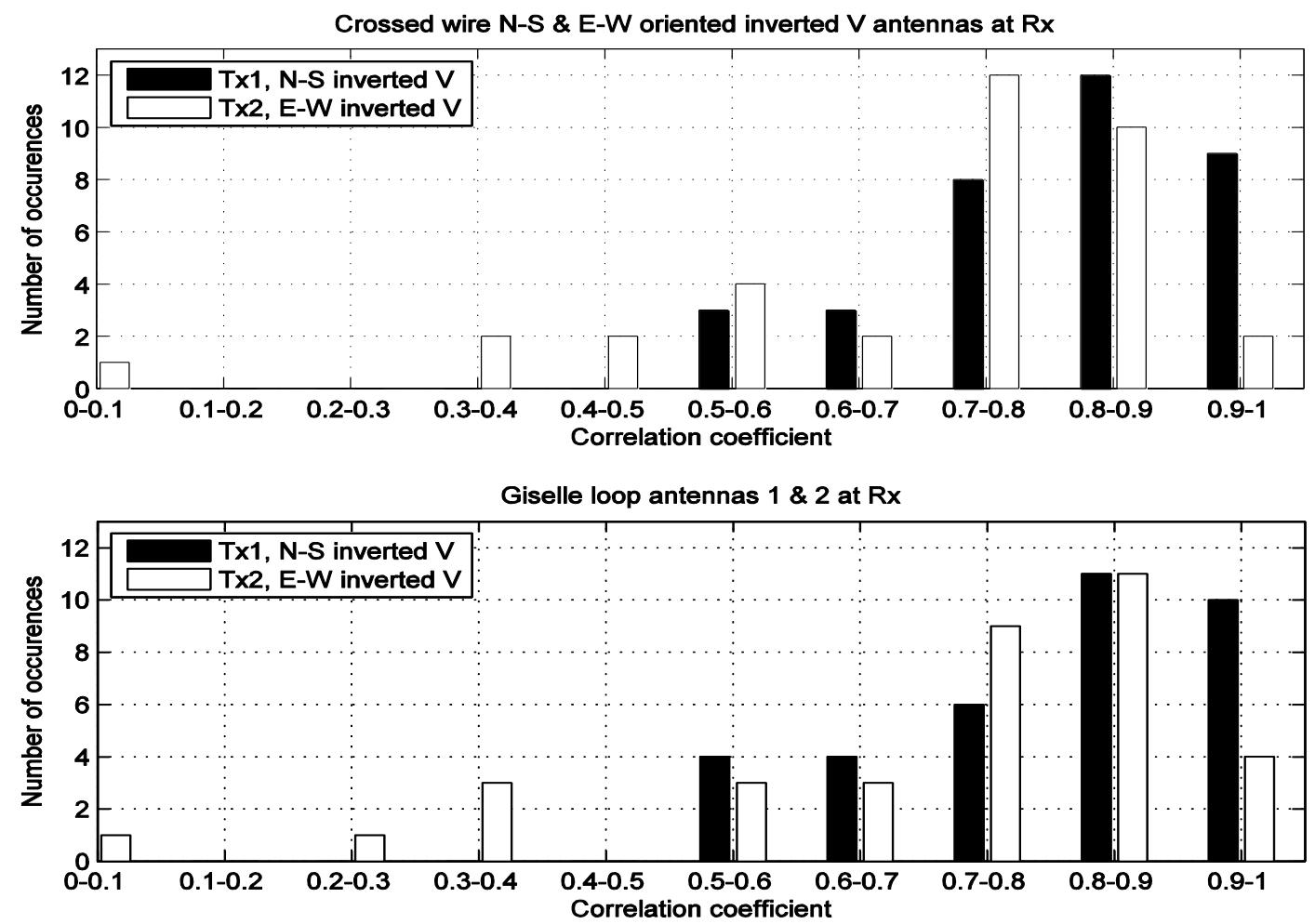

Figure 4.28 Envelop correlation coefficient histograms for crossed wire inverted Vs (top) and collocated Giselle lioops 1 \& 2 
of decorrelation by analyzing the results over a period of time.

The north-south and east-west oriented loop antennas in the XYZ array show a greater level of decorrelation as compared to the crossed wire inverted $\mathrm{V}$ wire antennas. The decorrelation provided is even greater for the north-south and horizontal loop antennas. The east-west and horizontal loop pair however are highly correlated and these differences could also be attributed to the relative phase pattern differences. The capacity comparison for individual one minute data acquisitions for crossed wire inverted V pair and the Giselle antennas 1 and 2 is shown in Figure 4.33. The capacity estimates are comparable with the Giselle antennas performing better in most of the instances.
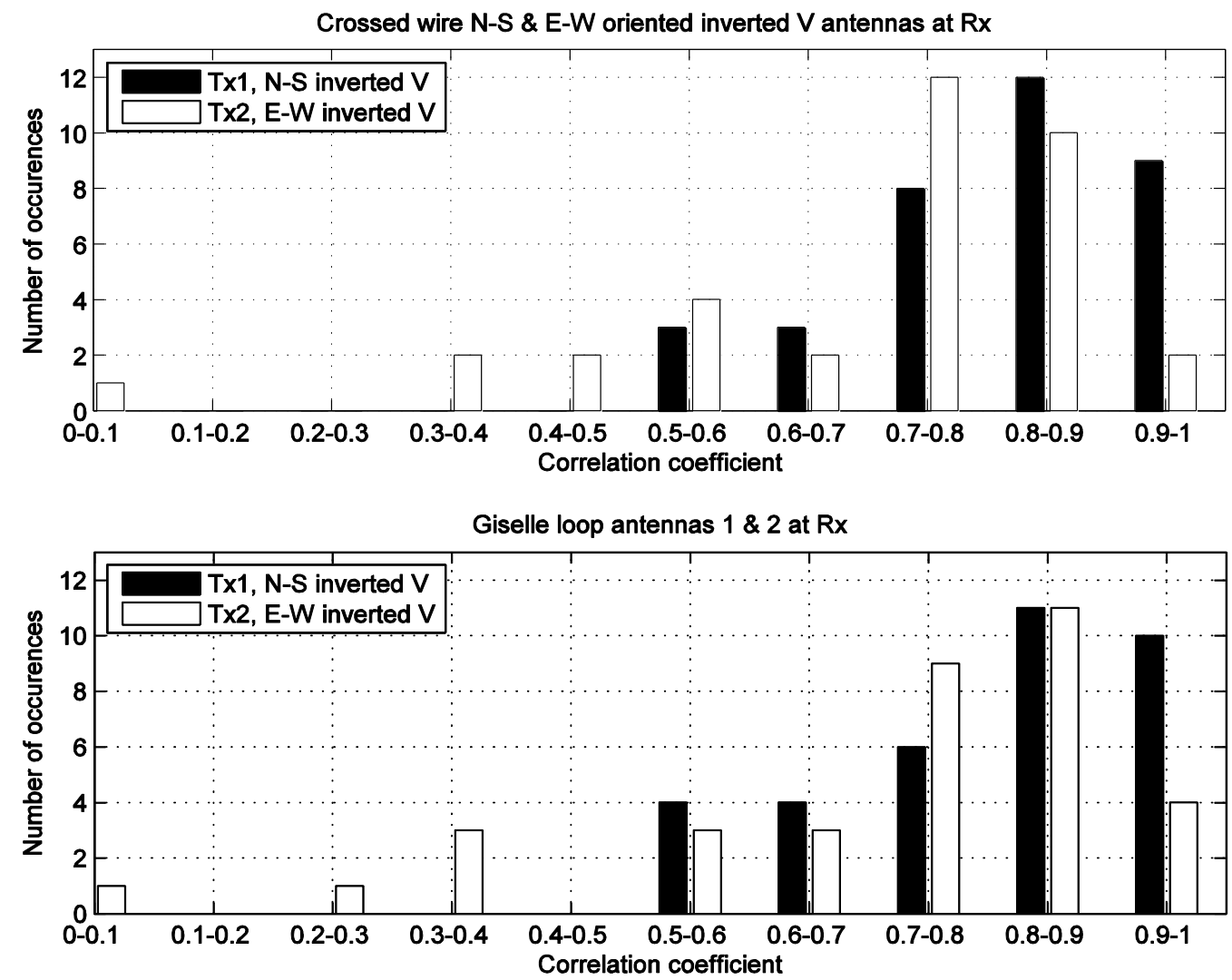

Figure 4.29 Envelope correlation coefficient histograms for crossed wire inverted Vs (t op) and collocated Giselle loops 1 and 2 

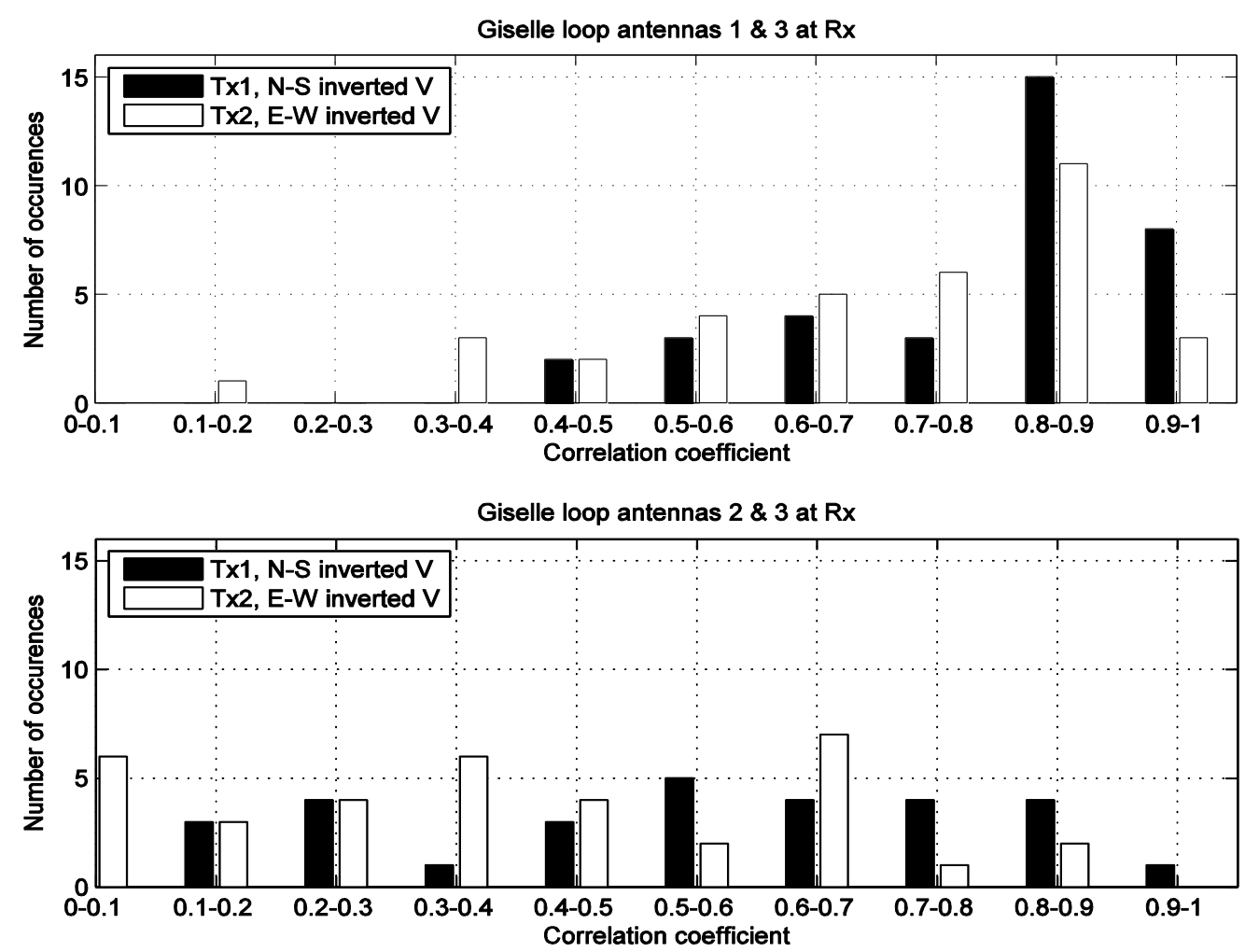

Figure 4.30 Envelope correlation coefficient histograms for collocated Giselle antennas $1 \& 3$, and $2 \& 3$
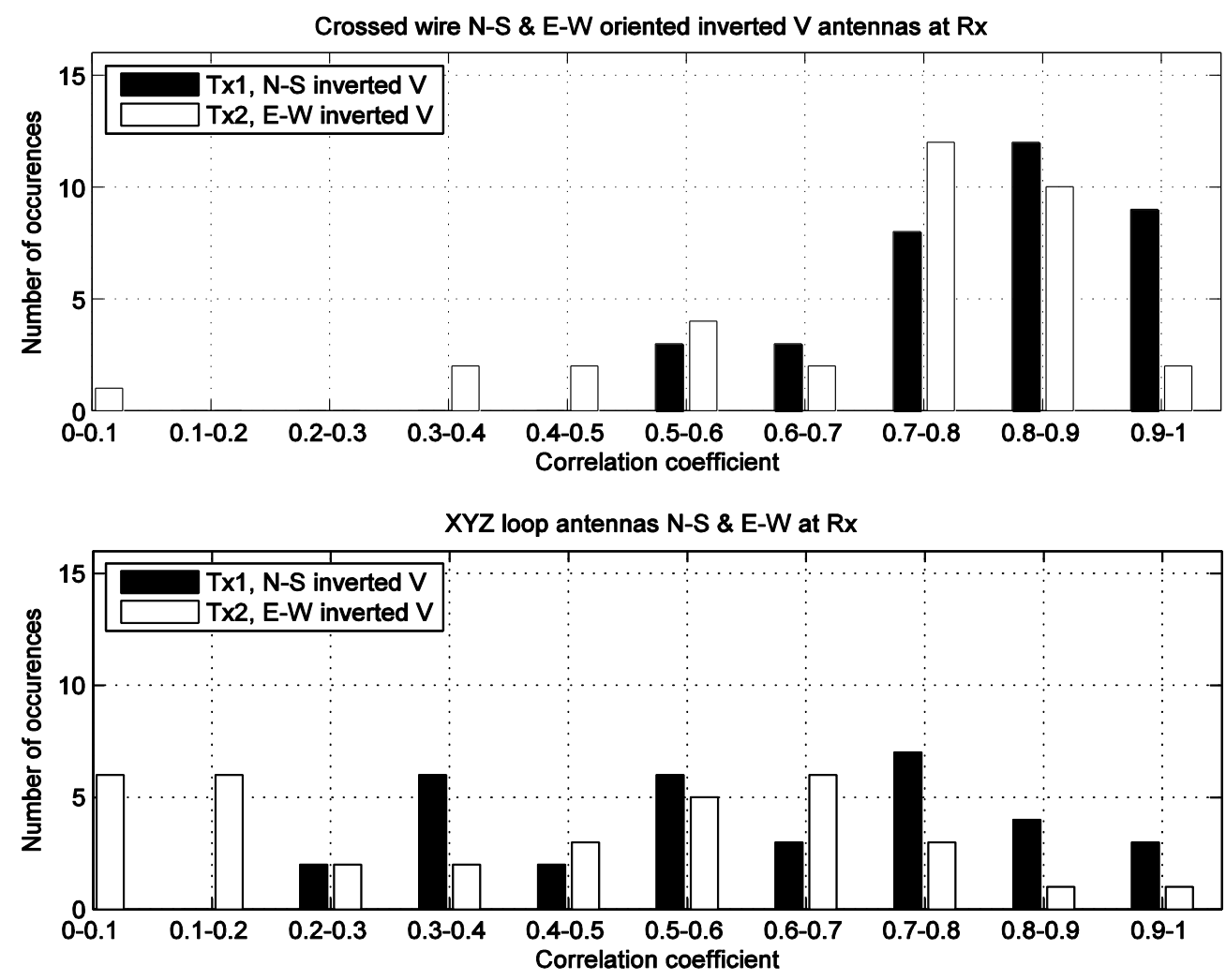

Figure 4.31 Envelope correlation coefficient histograms for crossed wire inver ted Vs (top) and collocated XYZ loops, nor th-south and east-west (bottom) 

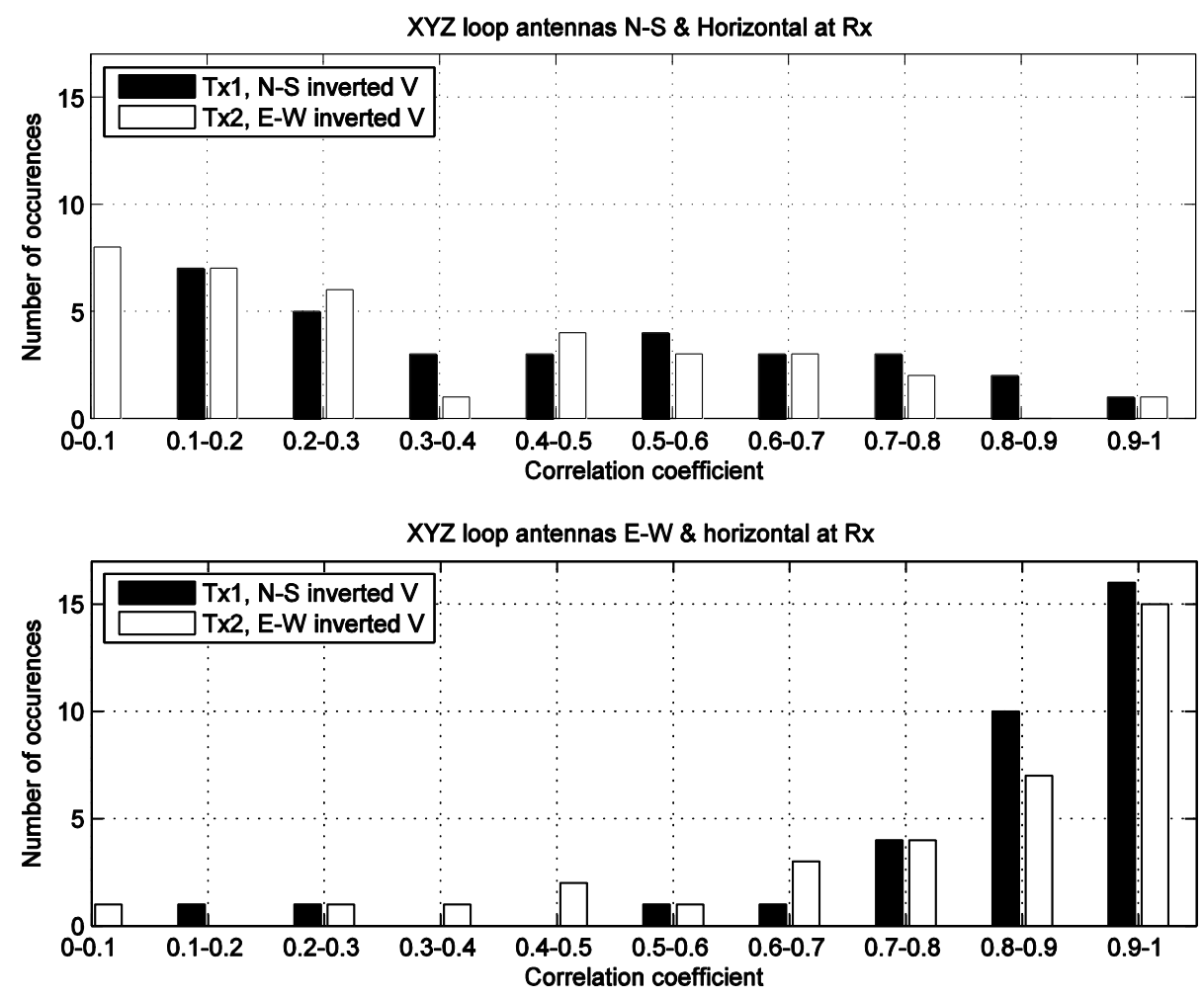

Figure 4.32 Envelope correlation coefficient histograms for collocated XYZloops, north- south \& horizontal (top) and east-west \& horizontal (bottom)
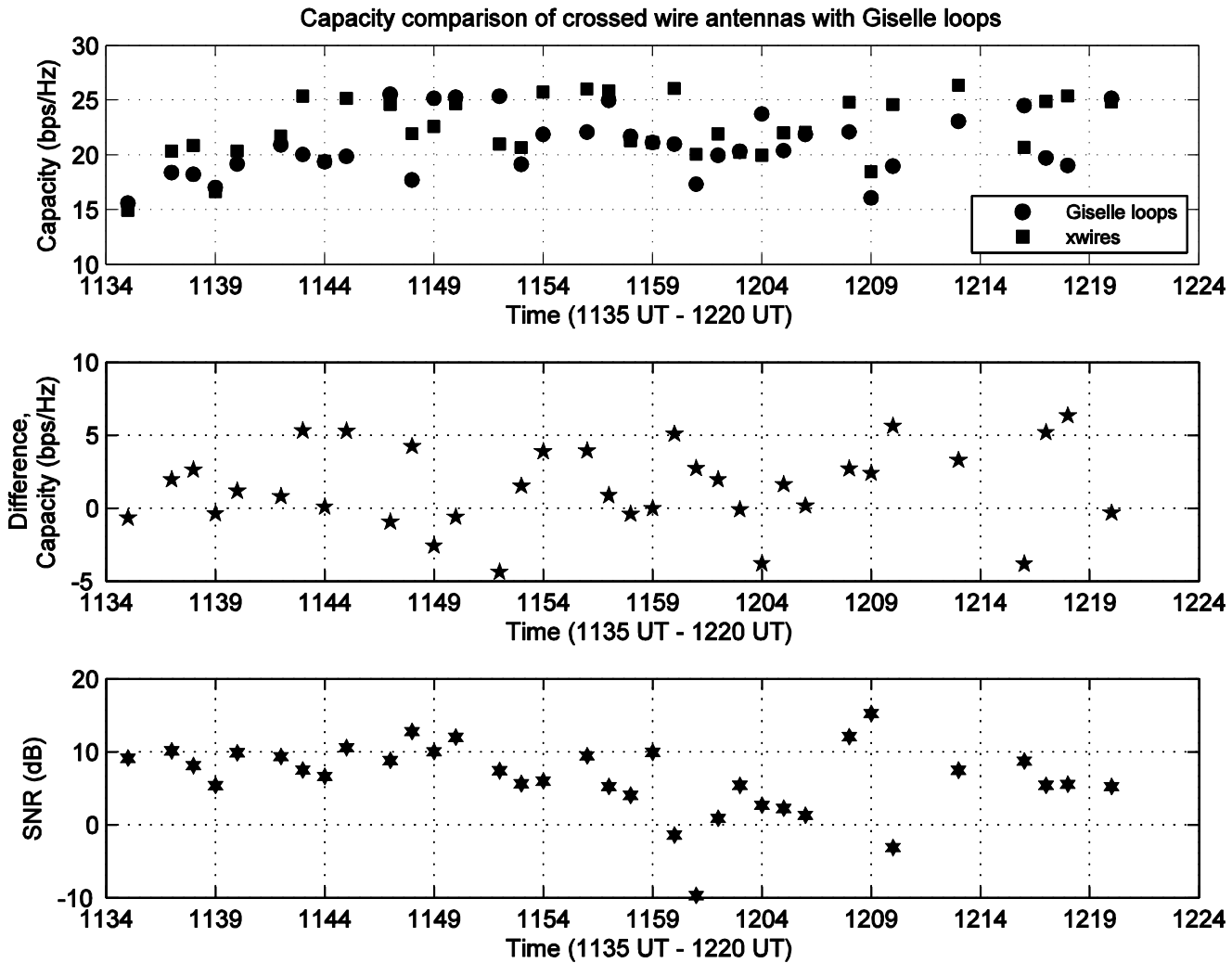

Figure 4.33 Comparis on of crossed inverted $V$ array with collocated Giselle loops $1 \& 2$ at $40 \mathrm{~dB}$ SNR 


\subsection{Summary of results for collocated antenna arrays}

The whole data set was analyzed to produce the summary of results as shown in Table 4.2. This gives a general overview of the performance of collocated antenna arrays irrespective of the propagation conditions. The XYZ loops and the Giselle loops can be seen to give comparable levels of decorrelation with the large crossed wire array. The antennas in the monopole array are however very much correlated and thus would not be suitable for an HF-MIMO implementation.

\begin{tabular}{|c|c|c|c|c|c|}
\hline & $\begin{array}{c}\text { Mean } \\
\text { correlation }\end{array}$ & $\begin{array}{l}\text { Standard } \\
\text { deviation }\end{array}$ & $\%$ values $<0.9$ & $\begin{array}{c}\% \text { values } \\
<0.7\end{array}$ \\
\hline \multicolumn{2}{|c|}{ Crossed wire Inverted Vs } & 0.68 & 0.28 & 74 & 42 \\
\hline \multicolumn{2}{|c|}{ Crossed Dipoles } & 0.75 & 0.30 & 53 & 31 \\
\hline \multirow{3}{*}{ XYZ loops } & N-S \& E-W & 0.60 & 0.4 & 73 & 45 \\
\hline & NS \& H & 0.67 & 0.32 & 71 & 41 \\
\hline & $E-W \& H$ & 0.61 & 0.35 & 76 & 48 \\
\hline \multirow{3}{*}{ Giselle } & G1 \& G2 & 0.67 & 0.39 & 58 & 35 \\
\hline & G1 \& G3 & 0.74 & 0.28 & 57 & 35 \\
\hline & G2 \& G3 & 0.66 & 0.41 & 57 & 36 \\
\hline \multirow{3}{*}{ Monopoles } & M1 \& M2 & 0.87 & 0.16 & 38 & 12 \\
\hline & M1 \& M3 & 0.90 & 0.13 & 30 & 13 \\
\hline & $\mathrm{M} 2 \& \mathrm{M} 3$ & 0.90 & 0.10 & 32 & 23 \\
\hline
\end{tabular}

Table 4.2 Summary of results for collocated antennas used in HF-MIMO experiments

\subsection{Summary}

The results from different experiments with spaced and collocated antenna arrays were presented in this chapter. Envelope correlation coefficients and capacity estimates were calculated for different sets of antenna pairs in the arrays. An L-shaped spaced, homogenous array consisting of 5 monopole antennas was used to study the suitability of using such arrays in MIMO applications. The correlation between the antennas in spaced homogenous arrays depends on the separation distances, and a minimum spacing of $\lambda / 2$ is quoted in literature for decorrelation [Jakes, 1974]. Thus the antennas were arranged to have the varying space between them ranging from less than $\lambda / 2$ to greater than $\lambda / 2$. It was determined that the correlation between pairs with smaller 
separation was higher than the ones with larger separation. Also the orientation of the spaced antennas also had an effect on the decorrelation. The array with axis towards the direction of propagation was more decorrelated than the antenna pairs perpendicularly oriented to the direction of arrival (Table 4.1). However it was found out that antennas must be separated by distances much greater than $\lambda / 2$ for spaced arrays to provide decorrelation consistently under varying ionospheric conditions.

Some novel collocated antenna configurations were used in different HF-MIMO experiments which reduce the size of the array to the dimensions of a single antenna. Such antenna arrays depend on polarization and pattern diversity to provide the decorrelation and hence may be used in HF-MIMO systems. Except for the dipole array, all other arrays were made of similar sensor elements with different orientations. This allowed the comparisons to be made between antennas with same electrical characteristics and the decorrelation is dependent only on how the antennas are oriented in the array. It has been shown that the crossed wire inverted $\mathrm{V}$ antenna pair performs consistently well in providing decorrelation between signals under a variety of ionospheric conditions. The capacity estimates also confirm the decorrelation results and appreciable increase is witnessed even under less favourable ionospheric conditions (first part of section 4.2.1). The drawback of using this antenna for MIMO applications is that it is still large for many applications (28 $\mathrm{m}^{2}$ area) and is cumbersome to set up. Different small and portable antennas were built with active electronics at Durham University and their performance compared with the crossed wire antennas. The 3 collocated monopoles were one such array but the antennas were not able to provide any appreciable decorrelation in the experiments. The results from all the campaigns using collocated antennas is presented in Table 4.2. All pairs in the arrays show good performance in terms of correlation except the monopole array 
which has highly correlated antenna elements. These results necessitate modelling antenna arrays for parameters which could be able to discriminate between the incoming multipath signals and hence provide decorrelation. The XYZ loops, collocated dipoles and the Giselle antenna arrays however gave comparable and sometimes better results than the crossed wires. All these antennas are very small (around $1 \mathrm{~m}^{2}$ area), portable and easy to set-up. This is very encouraging in terms of the practical usage of such antennas in HF-MIMO applications. 


\section{Antenna Modelling for HF-MIMO}

A variety of different antennas were used in HF-MIMO experiments and their correlations and capacity estimates calculated. In order to better understand the parameters providing decorrelation, antenna modelling was employed. Thus heterogeneous arrays could be developed whose performance in terms of decorrelation could be predicted like the case of spaced arrays. Since the decorrelation at the MIMO sensors depends on a number of factors based on antenna design (both Tx and Rx), placement, link geometry and channel conditions, all aspects have to be taken into account when looking for ways to design MIMO efficient collocated antennas.

\subsection{Antenna Modelling with Numerical Electromagnetic Code (NEC)}

If currents flowing through the antenna are known, electromagnetic field generated can be found, which can be represented in the spherical coordinate system. This EM field can be divided into reactive near field and radiated far field components. The far field can be represented in different ways. One of the ways to represent far field is in the form of absolute fields. In case of linearly polarized antennas E field can be decomposed into co-polar and cross-polar components; the ratio of these components indicates the polarization mismatch. The radiated far field of an antenna can be represented by linearly polarized components; $E_{\theta}$ and $E_{\varphi}$. Elliptically polarized antennas patterns can be better described in terms of right hand and left hand polarization components. $\mathrm{E}_{\text {left }}$ and $\mathrm{E}_{\text {right }}$.

The software used for modelling the antennas was Numerical Electromagnetic Code (NEC) version 2. NEC is a Fortran based program developed by the Lawrence Livermore Laboratories in the 1970s. NEC uses method of moments [Harrington, 
1967] to calculate a numerical solution to the integrals equations using Green's function. This method is suitable for wire antennas with diameter less than $0.05 \lambda$ for the solution of integral equations that determine the currents induced on the antenna elements. The input impedance and the electromagnetic fields at any arbitrary distance from the antenna can then be calculated from these currents. NEC uses electric field integral equation (EFIE) to predict the electromagnetic response of wire structures or grids and magnetic field integral equation (MFIE) is used to model smooth surface antennas.

NEC divides antenna elements into small segments and applies method of moment's integrals to solve for the properties of the antenna. The results of these calculations are added to determine the response of the antenna.

\subsubsection{Modelling guidelines for NEC}

The reliability of the output obtained from NEC models depends on the accuracy of the models and the adherence to the guidelines for the use of the software. An antenna in NEC is made up of elements which are then made up of wires. Wires are straight conductors which combine to form an element. Some antenna like the Yagi can have more than one element. Some important modelling guidelines for using NEC are [Cebik, 2001]:

- Each wire is divided into segments for applying the method of moments. A wire should have at least 20 segments per wavelength.

- Segment lengths should greater then wire diameter. To avoid errors segment lengths greater than 4 times the wire diameter were used.

- If possible all segment lengths should be made equal within a model. This was ensured in all the models. 
- Odd number of segments allows for the source to be placed at the centre and this convention was used in the models.

- For all the models the linear elements were made to lie parallel to the y-axis for ease of investigating the radiation patterns.

- The $\mathrm{x}$-axis was used for front to back dimensions with the part of the elements behind (with reference to the direction of the incoming signal) were taken as negative and in the front as positive.

- The height of the antenna above the ground was always taken on the z-axis

The accuracy of NEC model is checked by the segmentation test which ensures that the wires have been divided into enough number of segments for the method of moments to calculate currents properly. Segmentation level of the model is increased $50 \%$ at each iteration, until the results converge. The second method to determine the accuracy of the model is the average gain test whereby the free space 3-dimensional radiation pattern test is performed by omitting wire losses and resistive loads. The average gain under these conditions should be 1 with some tolerance level. These tests give a certain degree of confidence on the model. NEC has certain modelling limitations however the antennas modelled of HF-MIMO were not so complex to be affected by these.

The gain of the antenna is measured in $\mathrm{dBi}$ with respect to an isotropic radiator. NEC offers a number of choices about the ground underneath the antenna however it assumes it to be uniform. Average ground was used in the modelling of HF-MIMO antennas with a conductivity of $0.005 \mathrm{mho} / \mathrm{m}$ and dielectric constant of 13 . The choice was made as most of the experiments were carried out at a nominal frequency of $5 \mathrm{MHz}$ where the characteristics of ground spread over a large area can affect the performance of the antenna. Sommerfeld/Norton [Bubenik, 1977], [Norton, 1936] 
ground was used which increases the accuracy of ground effects at the cost of more processing power.

Typical NEC output is in text format. Sample outputs for a dipole antenna are shown in Figure 5.1, Figure 5.2 and Figure 5.3. The software application cocoaNEC was used to get the processed output in graphical format. It is a MAC OSX application which uses NEC-2 as the compute engine and allows the use of a tabular spreadsheet like interface for model input as well as a dedicated language for antenna modelling [Chen, 18 August 2011].

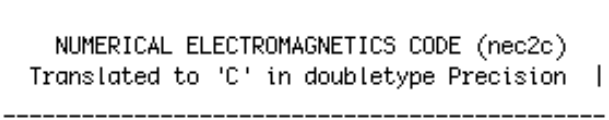

$$
\text { dipole 2012-03-10 14:27 }
$$

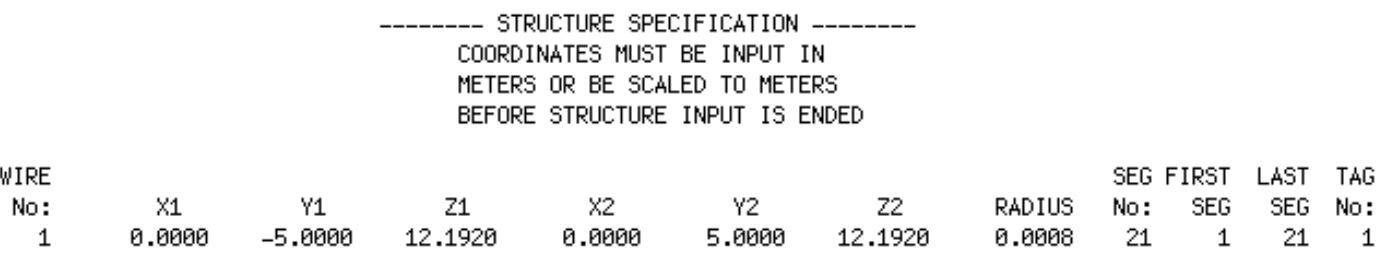

GROUND PLANE SPECIFIED.

WHERE WIRE ENDS TOUCH GROUND, CURRENT WILL BE INTERPOLATED TO IMAGE IN GROUND PLANE.

TOTAL SEGMENTS USED: 21 SEGMENTS IN A SYMMETRIC CELL: 21 SYMMETRY FLAG:

TATION DATA

COORDINATES IN METERS

I+ AND I- INDICATE THE SEGMENTS BEFORE AND AFTER I

\begin{tabular}{|c|c|c|c|c|c|c|c|c|c|c|c|}
\hline SEG & COORDIN & TES OF SE & 1 CENTER & SEGM & ORIENTAT & ON ANGLES & WIRE & \multicolumn{2}{|c|}{ CONNECTION } & DATA & TAG \\
\hline No: & $x$ & $Y$ & $z$ & LENGTH & ALPHÁ & BETÁ & RADIUS & I- & I & $\mathrm{I}+$ & 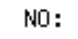 \\
\hline 1 & 0.0000 & -4.7619 & 12.1920 & 0.4762 & 0.0000 & 90.0000 & 0.0008 & 0 & 1 & 2 & \\
\hline 2 & 0.0000 & -4.2857 & 12.1920 & 0.4762 & 0.0000 & 90.0000 & 0.0008 & 1 & 2 & 3 & \\
\hline 3 & 0.0000 & -3.8095 & 12.1920 & 0.4762 & 0.0000 & 90.0000 & 0.0008 & 2 & 3 & 4 & \\
\hline 4 & 0.0000 & -3.3333 & 12.1920 & 0.4762 & 0.0000 & 90.0000 & 0.0098 & 3 & 4 & 5 & \\
\hline 5 & 0.0000 & -2.8571 & 12.1920 & 0.4762 & 0.0000 & 90.0000 & 0.0008 & 4 & 5 & 6 & \\
\hline 6 & 0.0000 & -2.3810 & 12.1920 & 0.4762 & 0.0000 & 90.0000 & 0.0008 & 5 & 6 & 7 & \\
\hline 7 & 0.0000 & -1.9048 & 12.1920 & 0.4762 & 0.0000 & 90.0000 & 0.0008 & 6 & 7 & 8 & \\
\hline 8 & 0.0000 & -1.4286 & 12.1920 & 0.4762 & 0.0000 & 90.0000 & 0.0008 & 7 & 8 & 9 & \\
\hline 9 & 0.0000 & -0.9524 & 12.1920 & 0.4762 & 0.0000 & 90.0000 & 0.0008 & 8 & 9 & 10 & \\
\hline 10 & 0.0000 & -0.4762 & 12.1920 & 0.4762 & 0.0000 & 90.0000 & 0.0008 & 9 & 10 & 11 & \\
\hline 11 & 0.0000 & 0.0000 & 12.1920 & 0.4762 & 0.0000 & 90.0000 & 0.0008 & 10 & 11 & 12 & \\
\hline 12 & 0.0000 & 0.4762 & 12.1920 & 0.4762 & 0.0000 & 90.0000 & 0.0008 & 11 & 12 & 13 & \\
\hline 13 & 0.0000 & 0.9524 & 12.1920 & 0.4762 & 0.0000 & 90.0000 & 0.0098 & 12 & 13 & 14 & \\
\hline
\end{tabular}

Figure 5.1 Sample nec2 output showing antenna coor dinate infor mation 
CURRENTS AND LOCATION

DISTANCES IN WAVELENGTHS

\begin{tabular}{|c|c|c|c|c|c|c|c|c|c|}
\hline EG & TAG & COORDIN & ES OF & ENTER & SEGM & & & MPS) - & \\
\hline do: & No: & $x$ & $\mathrm{Y}$ & $z$ & LENGTH & REAL & IMAGINARY & MAGN & HASE \\
\hline 1 & 1 & 0.0000 & -0.2236 & 0.5726 & 0.02236 & $8.5197 \mathrm{E}-04$ & $7.4099 \mathrm{E}-04$ & $1.1291 \mathrm{E}-03$ & 41.015 \\
\hline 2 & 1 & 0.0000 & -0.2013 & 0.5726 & 0.02236 & $2.3569 \mathrm{E}-03$ & $2.0645 \mathrm{E}-03$ & $3.1332 \mathrm{E}-03$ & 41.216 \\
\hline 3 & 1 & 0.0000 & -0.1789 & 0.5726 & 0.02236 & $3.7257 \mathrm{E}-03$ & $3.2879 \mathrm{E}-03$ & $4.9690 \mathrm{E}-03$ & \\
\hline 4 & 1 & 0.0000 & -0.1566 & 0.5726 & 0.02236 & $4.9805 \mathrm{E}-03$ & $4.4305 \mathrm{E}-03$ & $6.6659 \mathrm{E}-03$ & 41.655 \\
\hline 5 & 1 & 0.0000 & -0.1342 & 0.5726 & 0.02236 & 6.1103E-03 & $5.4819 \mathrm{E}-03$ & $8.2089 \mathrm{E}-03$ & \\
\hline 6 & 1 & .0000 & -0.1118 & 0.5726 & 0.02236 & 7.0 & $6.4284 \mathrm{E}-03$ & $9.5773 \mathrm{E}-63$ & 160 \\
\hline 7 & 1 & .0000 & -0.0895 & 0.5726 & 0.02236 & 7.9 & $7.2557 \mathrm{E}-03$ & $1.0750 \mathrm{E}-02$ & 2.449 \\
\hline 8 & 1 & .0000 & -0.0671 & 0.5726 & 0.02236 & & 7.951 & $1.171 \mathrm{bE}-62$ & 2.770 \\
\hline 9 & 1 & .0000 & -0.0447 & 0.5726 & 0.02236 & & 8.506 & $1.2440 \mathrm{E}-02$ & \\
\hline 10 & 1 & 0.0000 & -0.0224 & 0.5726 & 0.02236 & 9.3 & $8.9152 \mathrm{E}-03$ & $1.2934 \mathrm{E}-02$ & 43.572 \\
\hline 11 & 1 & 90 & & 0.5726 & & & & 1.3 & \\
\hline 12 & 1 & .0000 & 0.0224 & 0.5726 & 0.02236 & 9.3 & 8.915 & $1.2934 \mathrm{E}-02$ & 572 \\
\hline 13 & 1 & .0000 & 0.0447 & 0.5726 & 0.02236 & & 8.506 & $1.2440 \mathrm{E}-02$ & \\
\hline 14 & 1 & .0000 & 0.0671 & 0.5726 & .02236 & & 7.95 & $1.1710 \mathrm{E}-02$ & \\
\hline 15 & 1 & 0.0000 & 0.0895 & 0.5726 & 0.02236 & 7.93 & $7.2557 \mathrm{E}-03$ & $1.0750 \mathrm{E}-02$ & 42.449 \\
\hline 16 & 1 & 0900 & & & & & 6.428 & $9.5773 \mathrm{E}-03$ & \\
\hline 17 & 1 & 0.0000 & 0.1342 & 0.5726 & 0.02236 & 6.118 & $5.4819 \mathrm{E}-03$ & $8.2089 \mathrm{E}-63$ & 41.897 \\
\hline 18 & + & & & & & & & $6.6659 \mathrm{E}-03$ & \\
\hline 19 & 1 & & 0.1789 & 0.5726 & 0.02236 & 3.7 & $3.2879 \mathrm{E}-03$ & $4.9690 \mathrm{E}-03$ & 41420 \\
\hline 20 & 1 & & & & & 2.3 & & $3.1332 \mathrm{E}-03$ & 16 \\
\hline 21 & 1 & & & 26 & .02236 & $=-64$ & -64 & 1.1291E-03 & 41 \\
\hline
\end{tabular}

------- POWER BUDGET --------
INPUT POWER $=4.7346 E-03$ watts
RADIATED POWER= 4.7346 E- 03 Watts
STRUCTURE LOSS $=0.0000$ +00 Watts
$\begin{aligned} \text { NETWORK LOSS } & =0.0000 E+00 \text { Watts } \\ \text { EFFICIENCY } & =100.00 \text { Percent }\end{aligned}$

Figure 5.2 Sample nec2 output showing currents in the antenna and power budget

RANGE: $5.000000 \mathrm{E}+03$ METERS

EXP(-JKR)/R: 2.00000E-04 AT PHASE: -298.47 DEGREES

\begin{tabular}{|c|c|c|c|c|}
\hline AN & ES & & POWER GAIN & \\
\hline THETA & PHI & VERTC & HORIZ & TOTAL \\
\hline DEGREES & DEGREES & $\mathrm{DB}$ & DB & DB \\
\hline-90.00 & 0.00 & -999.99 & -999.99 & -999.99 \\
\hline-89.00 & 0.00 & -999.99 & -14.98 & -14.98 \\
\hline-88.00 & 0.00 & -999.99 & -9.00 & -9.00 \\
\hline-87.00 & 0.00 & -999.99 & -5.53 & -5 \\
\hline-86.00 & 0.00 & -999.99 & -3.09 & -3.8 \\
\hline-85.00 & 0.00 & -999.99 & -1.23 & -1.23 \\
\hline-84.00 & 0.00 & -999.99 & 0.26 & 0.26 \\
\hline-83.00 & 0.00 & -999.99 & 1.50 & 1. \\
\hline-82.00 & 0.00 & -999.99 & 2.54 & 2. \\
\hline-81.00 & 0.00 & -999.99 & 3.44 & 3.44 \\
\hline-80.00 & 0.00 & -999.99 & 4.21 & \\
\hline-79.00 & 0.00 & -999.99 & 4.88 & 4. \\
\hline-78.00 & 0.00 & -999.99 & 5.47 & \\
\hline-77.00 & 0.00 & -999.99 & 5.98 & 5. \\
\hline-76.00 & 0.00 & -999.99 & 6.43 & 6. \\
\hline-75.00 & 0.00 & -999.99 & 6.83 & 6.83 \\
\hline-74.00 & 0.00 & -999.99 & 7.17 & 7 \\
\hline-73.00 & 0.00 & -999.99 & 7.46 & \\
\hline-72.00 & 0.00 & -999.99 & 7.71 & 7 \\
\hline-71.00 & 0.00 & -999.99 & 7.91 & \\
\hline-70.00 & 0.00 & -999.99 & 8.08 & \\
\hline-69.00 & 0.00 & -999.99 & 8.22 & \\
\hline-68.00 & 0.00 & -999.99 & 8.32 & 8.32 \\
\hline-67.00 & 0.00 & -999.99 & 8.38 & \\
\hline-66.00 & 0.00 & -999.99 & 8.42 & an \\
\hline-65.00 & 0.00 & -999.99 & 8.43 & .43 \\
\hline-64.00 & 0.00 & -999.99 & 8.40 & \\
\hline-63.00 & 0.00 & -999.99 & 8.35 & .35 \\
\hline-62.00 & 0.00 & -999.99 & 8.27 & \\
\hline-61.00 & 0.00 & -999.99 & 8.17 & \\
\hline-60.00 & 0.00 & -999.99 & 8.03 & \\
\hline-59.00 & 0.00 & -999.99 & 7.87 & \\
\hline-58.00 & 0.00 & -999.99 & 7.68 & \\
\hline-57.00 & 0.00 & -999.99 & 7.47 & \\
\hline-56.00 & 0.00 & -999.99 & 7.22 & \\
\hline-55.00 & 0.00 & -999.99 & 6.95 & \\
\hline-54 ดด & ด ดด & -৭१९ १९ & 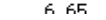 & \\
\hline
\end{tabular}

\begin{tabular}{|c|c|c|c|c|c|c|}
\hline \multicolumn{3}{|c|}{ RIZATION ---- } & \multicolumn{2}{|c|}{---- E(THETA) ---- } & \multicolumn{2}{|c|}{--- E(PHI) ----- } \\
\hline & TIL & SENS & MAGNITUDE & & & PHASE \\
\hline RATIO & DEGREES & & VOLTS/M & DEGREES & VOLTS/M & DEGREES \\
\hline 0.0000 & 0.00 & & $0.0000 \mathrm{E}+00$ & -298.47 & $5.5702 \mathrm{E}-15$ & -437.88 \\
\hline .0000 & 90.00 & LINEAR & $0.0000 \mathrm{E}+00$ & -298.47 & 1.8993E-65 & -257.96 \\
\hline 0.0000 & 90.00 & LINEAR & $0.0000 \mathrm{E}+00$ & -298.47 & 3.7816E-05 & -258.03 \\
\hline .0000 & 90.00 & LINEAR & $0.0000 \mathrm{E}+00$ & -298.47 & $5.6388 \mathrm{E}-05$ & -258.10 \\
\hline 0.0000 & 90.00 & LINEAR & $0.0000 \mathrm{E}+00$ & -298.47 & $7.4629 \mathrm{E}-05$ & -258.16 \\
\hline 0.0000 & 90.00 & LINEAR & $0.0000 \mathrm{E}+00$ & -298.47 & $9.2461 \mathrm{E}-05$ & -258.21 \\
\hline 0.0000 & 90.00 & LINEAR & $0.0000 \mathrm{E}+00$ & -298.47 & $1.0981 \mathrm{E}-04$ & -258.26 \\
\hline 0.0000 & 90.00 & LINEAR & $0.0000 \mathrm{E}+00$ & -298.47 & $1.2660 \mathrm{E}-04$ & -258.30 \\
\hline 0.0000 & 90.00 & LINEAR & $0.0000 \mathrm{E}+00$ & -298.47 & $1.4277 \mathrm{E}-04$ & -258.34 \\
\hline 0.0000 & 90.00 & LINEAR & $0.0000 \mathrm{E}+00$ & -298.47 & 1.5825E-64 & -258.37 \\
\hline 0.0000 & 90.00 & LINEAR & $0.0000 E+00$ & -298.47 & $1.7299 \mathrm{E}-04$ & -258.40 \\
\hline 0.0000 & 90.00 & LINEAR & $0.0000 \mathrm{E}+00$ & -298.47 & 1.8693E-64 & -258.42 \\
\hline 0.0000 & 90.00 & LINEAR & $0.0000 \mathrm{E}+00$ & -298.47 & $2.0002 \mathrm{E}-04$ & -258.43 \\
\hline 0.0000 & 90.00 & LINEAR & $0.0000 \mathrm{E}+00$ & -298.47 & $2.1222 \mathrm{E}-04$ & -258.43 \\
\hline 0.0000 & 90.00 & LINEAR & $0.0000 \mathrm{E}+00$ & -298.47 & $2.2350 \mathrm{E}-04$ & -258.43 \\
\hline 0.0000 & 90.00 & LINEAR & $0.0000 \mathrm{E}+00$ & -298.47 & $2.3381 \mathrm{E}-64$ & -258.42 \\
\hline 0.0000 & 90.00 & LINEAR & $0.0000 \mathrm{E}+00$ & -298.47 & $2.4313 \mathrm{E}-04$ & -258.41 \\
\hline 0.0000 & 90.00 & LINEAR & $0.0000 \mathrm{E}+00$ & -298.47 & $2.5145 \mathrm{E}-04$ & -258.38 \\
\hline & & LINEAR & $0.0000 \mathrm{E}+00$ & -298.47 & $2.5875 E-04$ & -258.35 \\
\hline 0.0000 & 90.00 & LINEAR & $0.0000 \mathrm{E}+00$ & -298.47 & $2.6501 \mathrm{E}-04$ & -258.31 \\
\hline & 90.00 & LINEAR & $0.0000 \mathrm{E}+00$ & -298.47 & $2.7025 \mathrm{E}-04$ & -258.26 \\
\hline 0.0000 & 90.00 & LINEAR & $0.0000 \mathrm{E}+00$ & -298.47 & $2.7445 \mathrm{E}-04$ & -258.20 \\
\hline & 90.00 & LINEAR & $0.0000 \mathrm{E}+00$ & -298.47 & $2.7762 \mathrm{E}-04$ & -258.13 \\
\hline 0.0000 & 90.08 & LINEAR & $0.0000 \mathrm{E}+00$ & -298.47 & $2.7979 \mathrm{E}-04$ & -258.06 \\
\hline 0.0 & 90.00 & LINEAR & $0.0000 \mathrm{E}+00$ & -298.47 & $2.8096 \mathrm{E}-04$ & -257.97 \\
\hline & 90.00 & LINEAR & $0.0000 \mathrm{E}+00$ & -298.47 & $2.8116 \mathrm{E}-04$ & -257.86 \\
\hline 0.0 & 90.00 & LINEAR & $0.0000 \mathrm{E}+00$ & -298.47 & $2.8041 \mathrm{E}-04$ & -257.75 \\
\hline & 90.00 & LINEAR & $0.0000 \mathrm{E}+00$ & -298.47 & $2.7875 \mathrm{E}-04$ & -257.62 \\
\hline 0.8 & 90.00 & LINEAR & $0.0000 \mathrm{E}+00$ & -298.47 & $2.7621 \mathrm{E}-04$ & -257.48 \\
\hline & 90.00 & LINEAR & $0.0000 \mathrm{E}+00$ & -298.47 & $2.7283 \mathrm{E}-04$ & -257.33 \\
\hline & 90.00 & LINEAR & $0.0000 \mathrm{E}+00$ & -298.47 & $2.6864 \mathrm{E}-04$ & -257.15 \\
\hline & 90.00 & LINEAR & $0.0000 \mathrm{E}+00$ & -298.47 & $2.6369 \mathrm{E}-04$ & -256.96 \\
\hline & 90.00 & LINEAR & $0.0000 \mathrm{E}+00$ & -298.47 & $2.5802 \mathrm{E}-04$ & -256.75 \\
\hline & 90.00 & LINEAR & $0.0000 \mathrm{E}+00$ & -298.47 & $2.5169 \mathrm{E}-04$ & -256.51 \\
\hline & 90.00 & LINEAR & $00 \mathrm{E}+06$ & -298.47 & $2.4473 \mathrm{E}-04$ & -256.26 \\
\hline & & LINEAR & $E+60$ & 17 & $2.3721 \mathrm{E}-04$ & -25 \\
\hline & & & & 17 & & -255 \\
\hline
\end{tabular}

Figure 5.3 Sample nec2 output showing radiation pattern details 


\subsection{Antenna diversity in HF-MIMO}

The ionosphere causes the incident electromagnetic wave to be split into $\mathrm{O}$ and $\mathrm{X}$ modes in addition to the multipath due to the reflections from different layers. With antenna diversity the radiation patterns of the antennas can be altered to receive complementary multipath signals as well as providing polarization filtering effect [Bisiaux and Bertel, 2000]. A bivectorial model has been proposed which gives the effect of propagation, antennas and link geometry on a HF narrow band signal [Bertel and Lemur, 2002].

$$
s_{r k}(t)=A_{k} m\left(t \tau_{g k}\right) e^{j \omega_{r k}\left(t-\tau_{p k}\right)}
$$

This equation represents the signal multipath and multi-mode components incident on the receiving antennas when a signal $m(t) e^{j \omega_{o} t}$ is sent. In this equation $s_{r k}(t)$ is the incident signal, $A_{k}$ represents the path loss for $\mathrm{k}^{\text {th }}$ mode, $\tau_{g k}$ is the group delay, $\tau_{p k}$ is the phase delay and $\omega_{r k}$ is the angular frequency with Doppler effects included.

For a MIMO system this response of the channel to the transmitted signal will be affected by the transmitting and receiving antennas thus the signal received at antenna $\mathrm{i}$ trans mitted by antenna 1 can be written as:

$$
X_{r l i}(t)=G_{l k} e^{j \gamma_{l k}} F_{i k} e^{j \delta_{i k}} S_{r k}(t) n_{i}(t)
$$

Where $X_{r l i}(t)$ is the waveform at the output of receive antenna $i$ for the transmit antenna $1, \quad G_{l k} e^{j \gamma_{l k}}$ and $F_{i k} e^{j \delta_{i k}}$ represent how the transmit antenna excites the mulipath mode $\mathrm{k}$ and the receive antennas response to the incoming $\mathrm{k}^{\text {th }}$ component and $n_{i}(t)$ is the noise vector for receive antenna $\mathrm{i}$. Thus the decorrelation between the signals would depend not only on the independence of multipath components but also on the response of transmit and receive antennas. This response will be a function of the angle of arrival of the incoming signal and its polarization. The polarization of a 
signal is determined by polarization ratio $\eta$ and the tilt angle $\alpha$. In the case of HF the $\mathrm{O}$ and $\mathrm{X}$ modes are elliptically polarized and similar except that these are rotating in opposite directions. Thus the HF incoming component can be divided into two parts and the response of the antenna in terms of each of these modes would depend on just the angle of arrival of the incoming wavefield.

Thus in case of HF collocated antennas three factors can influence the correlation between the signals on antennas:

1) The radiation patterns of the individual antennas in the collocated array whereby each antenna receives multipath components arriving from different elevation and azimuthal directions.

2) The differential phase variations in the individual antennas in the array.

3) The response of the antenna favouring $\mathrm{O}$ or $\mathrm{X}$ mode components of the HF wavefield.

\subsection{Modelling of collocated HF-M IMO antennas}

The collocated antennas in different orientations cause significant decorrelation. This decorrelation can be explained by how antennas, arranged in a structure excite the ionosphere differently and result in the reduced correlation at the receivers. Thus it is logical to assume that transmit and receive antennas will have a major role to play in achieving the objectives of MIMO and need to be modelled accordingly. The aim of this modelling was to indicate the factor that causes the decorrelation and then model antennas for which decorrelation results could be predicted. One of the problems that may arise in the analysis of modelled collocated antennas is that each antenna is an independent entity as well as part of the array. In NEC-2 models we have to consider the other antennas as passive elements when finding the results for a single antenna in 
the array. One important factor, creating the diversity in collocated arrays is the radiation pattern of the antennas. The far field pattern of an antenna is described by its E field as:

$$
E=\dot{\hat{u}} E(\theta, \phi) e^{j \psi(\theta, \phi)} \frac{e^{-j k r}}{r}
$$

Where $E(\theta, \phi)$ is the magnitude of the radiation pattern in the $\theta$ (elevation), $\phi$ (azimuth) direction, $e^{j \psi(\theta, \phi)}$ represents the phase component, $\mathrm{k}$ is the wave number and $r$ is the distance. The phase pattern of antenna in an array depends on the configuration of the array, fringing effects and mutual coupling [Kim et al., 2004]. The relative far field phase pattern is used in radar and direction finding applications. The phase responses have been shown to estimate direction of arrival using linear antenna arrays [Winter, 1982]. Since in the case of collocated arrays the same wavefield with multipath components is incident on all individual antennas the relative phase difference between antennas far field patterns could be attributed to cause the decorrelation. To validate this assumption the NEC-2 models for the arrays used in HF-MIMO experiments were built and phase responses analyzed. The phase information from NEC-2 modelling software has been shown to be reasonably accurate and in good agreement with the measured results [Jenkins and Petrie, 1997]

The parameters involved in creating antenna diversity patterns can be quantified by analysing the results from antenna modelling. The modelling of antennas was carried out in NEC-2 and the experimental conditions were replicated to the extent possible. The fact that similar antennas in different orientations were used in the collocated arrays simplifies the modelling because the modelling errors would be reduced as all antennas will have the same electrical characteristics. 
In order to reduce the size of the arrays electrically small antennas were used with active components. Loops are inexpensive antennas easily constructed and simple to set- up. These come in various shapes and configurations. The electrically small loop antenna has a circumference less than $\lambda / 10$ and radiates electromagnetic fields which are similar to the infinitesimal magnetic dipole [Balanis, 1982]. Electrically small loops have low efficiency generally and are therefore mostly employed at the receiver. At low HF frequencies below $10 \mathrm{MHz}$ efficiency of the antennas is not a problem as the noise also gets attenuated and SNR value stays constant hence making these very useful receiving antennas. The power in the near field of a loop antenna is mainly reactive and that in the far field regions is mainly resistive. The far field of the square loop is defined as

$$
E_{\varphi}=\eta \frac{\pi S I_{0} e^{-j k r}}{\lambda^{2} r} \sin \theta
$$

Where $\eta$ is the intrinsic impedance for free space, $S$ is the area of the loop, $I_{0}$ is the initial current, $k$ is the wave number and $r$ is the distance.

The magnetic field of the loop is defined as

$$
H_{\theta}=-\frac{\pi S I_{0} e^{-j k r}}{\lambda^{2} r} \sin \theta
$$

\subsection{Modelling results for crossed wire inverted $V$ antennas}

This array consisted of two end fed crossed wire inverted V antennas arranged in the north-south and east-west directions. The dimensions of this array are explained in Chapter 3. The north-south wire was aligned approximately in the direction of transmission as shown in Figure 5.4. The antenna is broadband, designed to operate over the whole HF band. Each wire of the antennas was divided into 29 segments and the diameter of the wire was AWG \# 14. The north-south antenna was fed at the base 
of the wire opposite to the transmitter and the east-west was fed at the end towards the east. Although the measured resistances varied, the antennas were modelled with a 300 ohm series resistance at the other ends.

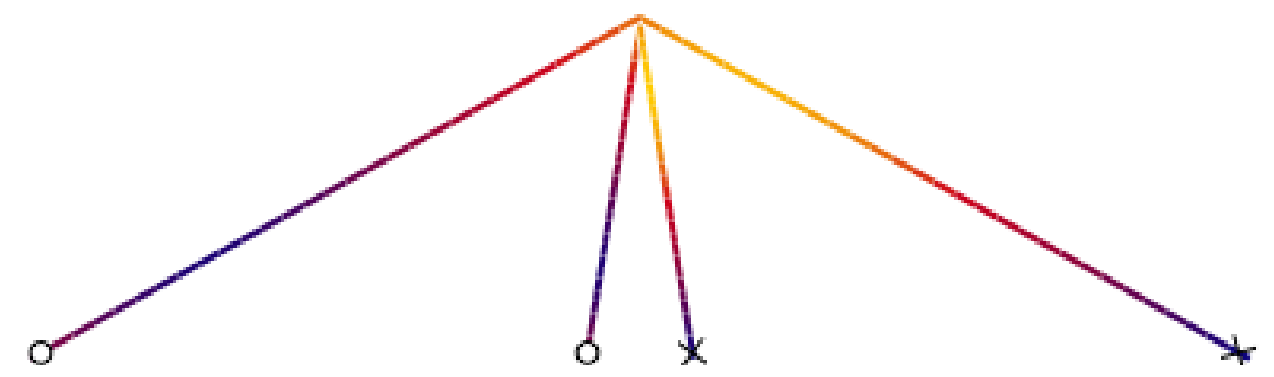

Figure 5.4 crossed wires inverted $V$ model

The NEC2 model of the antennas was run separately for north-south and eastwest wire by using the source for the antennas for which the output is desired. The sliced two dimensional radiation plots for north-south antenna are shown in Figure 5.5 $\left(0^{\circ}\right.$ azimuth and $5^{\circ}$ elevation-similar for all the antennas). Similar plots for east-west wire antenna are shown in Figure 5.6. The elevation pattern does not change much for both the north-south and east-west antennas; however the north-south antenna has more gain in the direction of transmission. Thus in terms of antenna diversity caused due to the antennas receiving different multipath components, this configuration would not work. However this antenna was used in many experimental campaigns with good decorrelation results as shown in Chapter 4. To delve into the cause of decorrelation the phase patterns of the two antennas in the array were analysed. For the geometry of the HF link between Durham and Leicester it is deduced that the one hop F2 mode will be arriving at the receiving antennas at approximately $70^{\circ}$ of elevation. Similarly the E layer reflections will be incident on the receiving antennas at around $30^{\circ}$. The elevation phase patterns for the two crossed wire pairs at $0^{\circ}$ azimuth are shown in Figure 5.7. The phase values have been scaled to meet at the right hand side of the figure and the phase for the east-west inverted $\mathrm{V}$ is forced to start at $0^{\circ}$. Since we are only interested 
in the slope and the relative difference this does not effect the results and improves readability. We can see that the slopes of the two patterns are inverted and there is a differential phase between the two. For example if we have a high angle ray from F2 layer coming at $70^{\circ}$ elevation angle (zero azimuth) and a low angle ray from E layer incident on the antenna array at $30^{\circ}$ (zero azimuth) then the differential phase comes out to be of the value of $47.14^{\circ}$. Thus the two signals incident on the collocated crossed wire antennas will give the phase responses which have a phase difference of $47.14^{\circ}$. The correlation between two cosine waves separated by this angle results in a correlation coefficient of 0.68 . Thus the decorrelation provided by the crossed wire antenna pair is likely to be introduced by the phase patterns with opposite slopes. As the incident waves are not expected to arrive scattered in the azimuth directions, only the angles close to zero are important in this analysis. However in order to determine the changes with the rotation of the antennas in the azimuthal plane the phase patterns between $-40^{\circ}$ and $40^{\circ}$ are shown in Figure 5.7. Since the antennas are symmetrical, plots for the other half of the azimuthal plane are not shown.
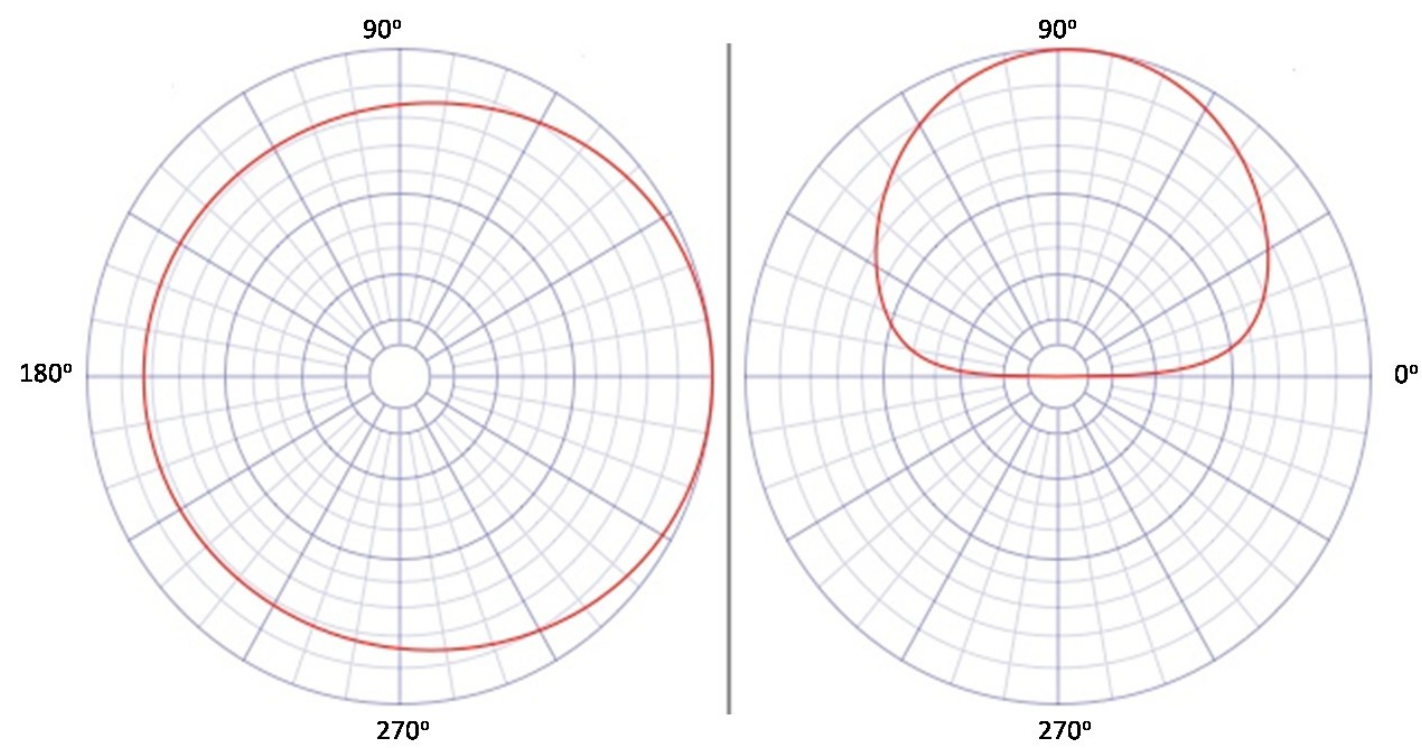

Figure 5.5 Nor th-s outh inverted V antennas elevation (right) and azimuth (left) pl ot 

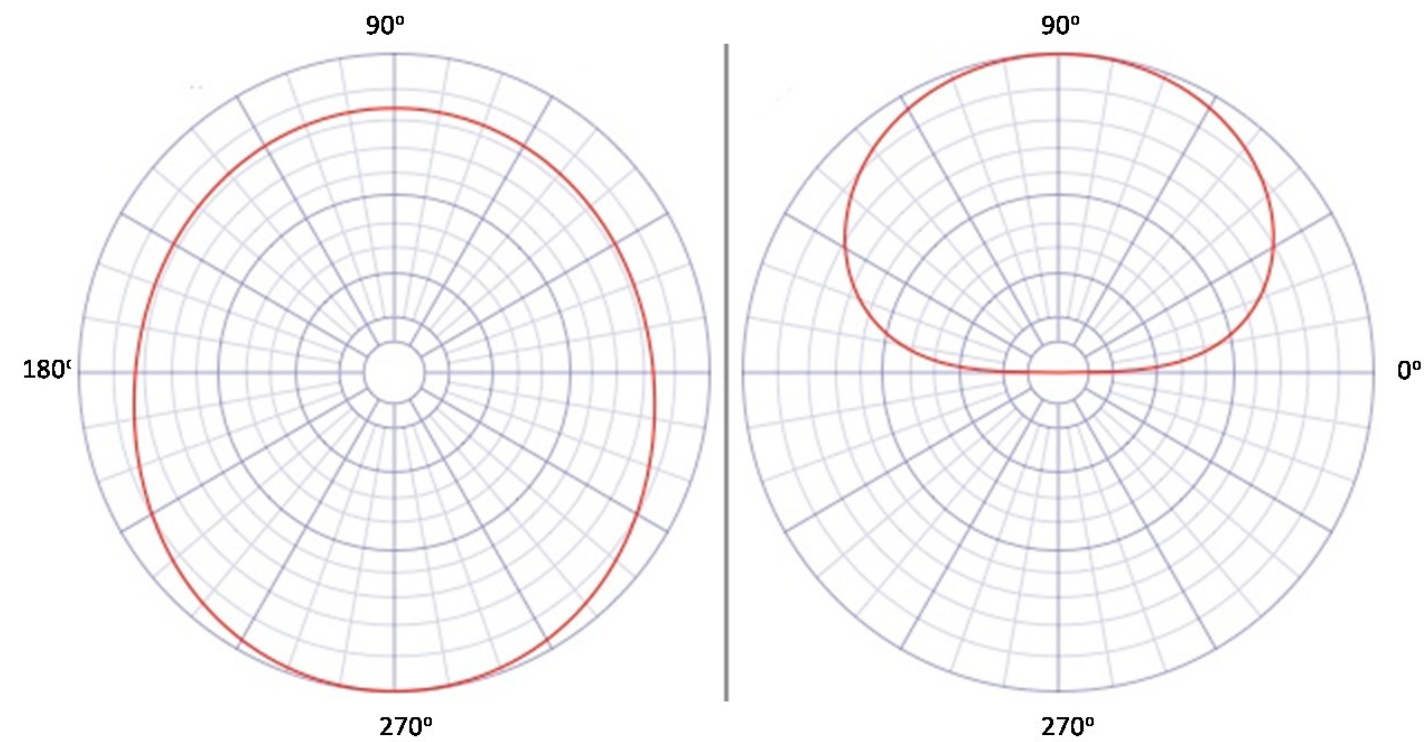

Figure 5.6 East-west inverted $V$ antennas ele vation (right) and azimuth (left) pl ot

The plot for a different frequency of $4.4455 \mathrm{MHz}$ used in some of the campaigns is shown in the bottom right of Figure 5.7. It is evident that the change in frequency will have an effect on the phase response of the antenna and thus its performance re garding the reception of de-correlated signals.

\subsubsection{Comparison of simulations with experiments}

To determine the link between the modelling results and the experimental findings a campaign carried out on 1 July 2008 was selected and data grouped into two parts depending on the mode availability and height of reflection. The campaign used two spaced monopoles, crossed wire inverted Vs and collocated monopoles at the receiver but for this analysis we will just consider the two crossed wire inverted V antennas.

Vertical ionograms at Chilton are available every 10 minutes. Chilton ionosonde is located $100 \mathrm{~km}$ south of the receiver site at Bruntingthorpe, Leicester. Transmission curves for the frequency used were superimposed on the ionograms to determine the ionospheric conditions experienced by the transmission. Although these only give an indication of the modes likely to be present and not the exact number of 

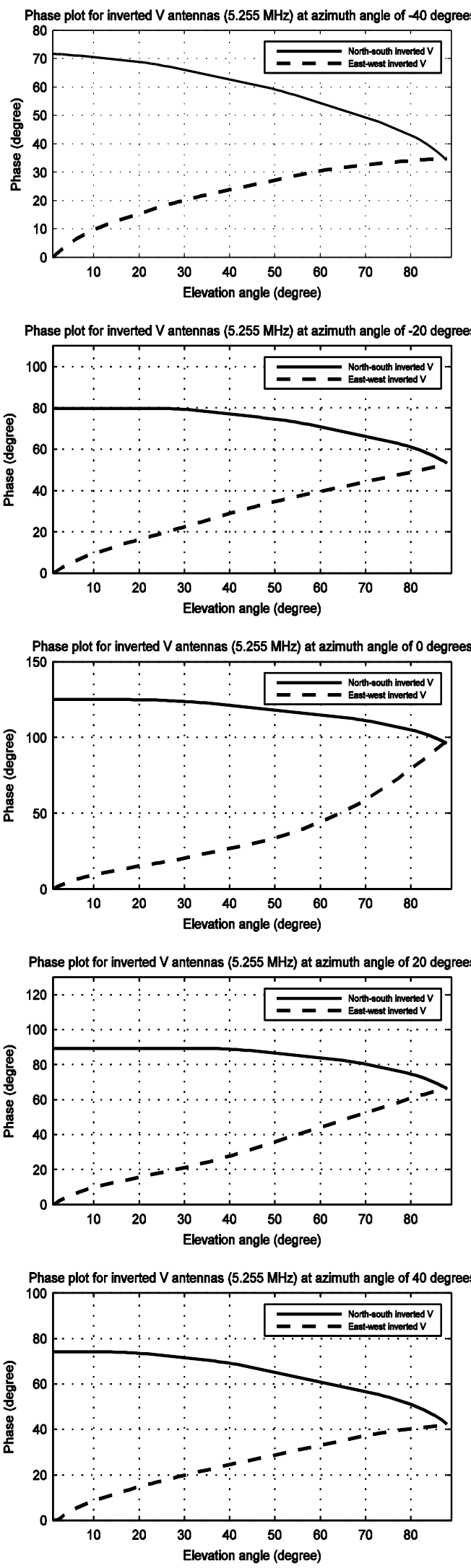
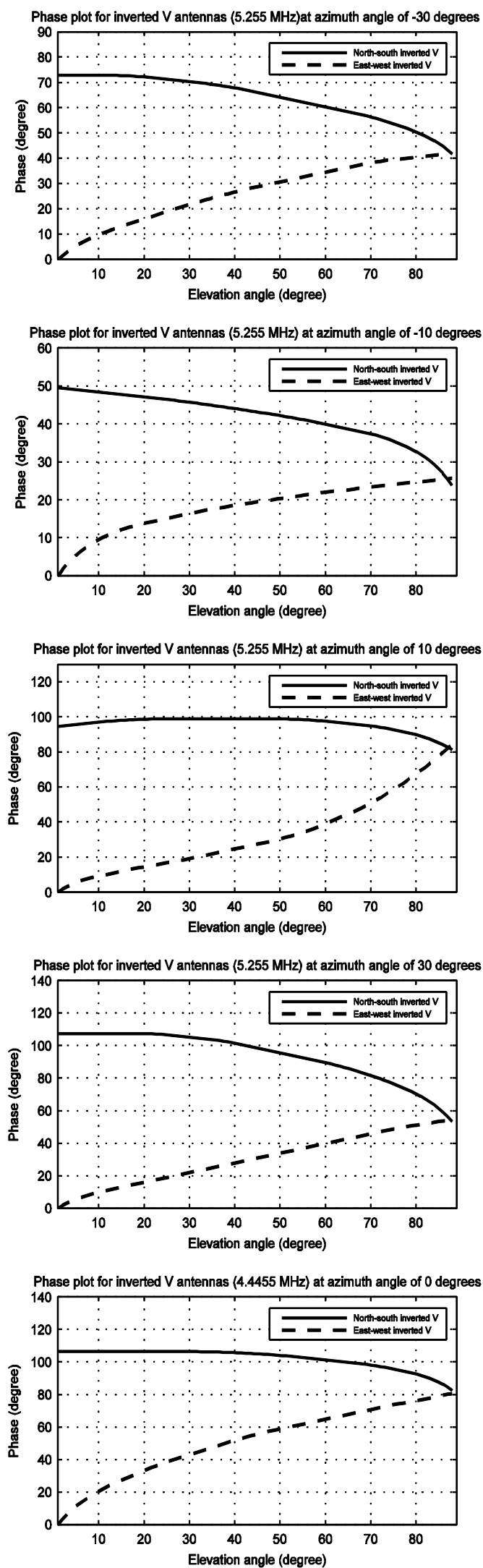

Figure 5.7 Phase patterns for north-south and east-west oriented inverted $\mathrm{V}$ antennas 
components actually available at a given time, they are nevertheless very helpful in providing general information about the state of the ionosphere.

The first part of the campaign consisted of files taken from 1347 UT-1421 UT and the second part from 1432 UT-1521 UT. The number of modes available and their approximate heights are given in the Table 5.1. The difference in heights between the modes in part $\mathrm{A}$ is less than that in part $\mathrm{B}$. One with modes arriving at the receiver with low angular (in terms of elevation) difference and the other with a higher angular difference. The phase difference between these two angles gives the phase response of the antennas to the incoming multipath signal. The level of decorrelation in the antenna array will depend on the difference in these phase response values. multipath wavefront impinging on each antenna made up of the components with maximum and minimum elevation angles. The degree of this phase decorrelation will decrease as the difference in angle of the impinging wavefront decreases and thus the decorrelation between antennas will fluctuate depending not only on the multipath components available but also on their relative elevation angles.

\begin{tabular}{|c|c|c|c|c|c|}
\hline \multirow{5}{*}{ Part A } & Time (UT) & $\begin{array}{c}\text { Number } \\
\text { of } \\
\text { Modes }\end{array}$ & Height (km) & $\begin{array}{c}\text { Phase } \\
\text { response } \\
\text { difference }\end{array}$ & $\begin{array}{l}\text { Average } \\
\text { correlation }\end{array}$ \\
\hline & 1350 & 2 & 120,230 & $21.26^{\circ}$ & \multirow{4}{*}{$\begin{array}{l}T \times 1=0.87 \\
T \times 2=0.85\end{array}$} \\
\hline & 1400 & 2 & 120,250 & $24.42^{\circ}$ & \\
\hline & 1410 & 2 & 120,240 & $22.82^{\circ}$ & \\
\hline & 1420 & 2 & 110,210 & $20.04^{\circ}$ & \\
\hline \multirow{6}{*}{ Part B } & 1430 & 3 & $\begin{array}{c}120,220 \\
440\end{array}$ & $45.85^{\circ}$ & \multirow{6}{*}{$\begin{array}{l}T \times 1=0.60 \\
T \times 2=0.47\end{array}$} \\
\hline & 1440 & 2 & 130,420 & $40.76^{\circ}$ & \\
\hline & 1450 & 2 & 110,400 & $43.14^{\circ}$ & \\
\hline & 1500 & 2 & 120,390 & $41.39^{\circ}$ & \\
\hline & 1510 & 3 & $120(2), 400$ & $41.39^{\circ}$ & \\
\hline & 1520 & 2 & 120,400 & $41.39^{\circ}$ & \\
\hline
\end{tabular}

Table 5.1 A comparis on of the effect of the phase response of the crossed wire antennas on correlation under the difference in the mode availability 


\subsection{Modelling results for Giselle collocated loop antennas}

The Giselle antenna comprises three electrically small square loops which are arranged symmetrically such that their phase centres are coincident. The base and the top form equilateral triangles which are inverted with reference to each other. The feed points of antennas lie at the centre of the bottom wire.

This antenna array was modelled for a frequency of $5 \mathrm{MHz}$. Each wire was divided into 29 segments and a wire diameter of AWG \#14 was used which approximated the antenna wire dimensions. Average ground was used to model this antenna.

The radiation patterns of the antennas were found to be different hence the loops in this arrays will be able to provide decorrelation on the basis of receiving multipath components from different layers of the ionosphere. The elevation and azimuth plots of the Giselle loop 1 are plotted in Figure 5.9. This antenna has its maximum gain between $30^{\circ}$ and $40^{\circ}$ elevation, therefore it will favour the multipath component arriving at these angles. The second Giselle antennas elevation and azimuth slices are shown in Figure 5.10. This antenna is oriented such that its maximum gain lies on the other side of zenith. The third Giselle antennas radiation patterns are shown in Figure 5.11. This antenna has its maximum gain at the angle of $30^{\circ}$ and thus will favour the lower angle multipath component. The gain values for the Giselle antennas between $30^{\circ}$ and $70^{\circ}$ are shown in Table 5.2. We can observe the variations in the gain of these antennas at various angles. This shows that the antennas will be receiving multipath components with different attenuation and hence will favour one component over the other. This will result in decorrelation between the signals received at antenna elements of Giselle array. The phase responses of Giselle antennas for different azimuth angles are plotted in Figure 5.12. Again we see that the phase pattern changes 
with the second Giselle antenna having the lowest slope. The values for the gain between $30^{\circ}$ and $70^{\circ}$ are shown in Table 5.2.

Thus the three antennas will introduce decorrelation based on their radiation patterns by receiving multipath components with different weighting factors applied and due to the varying phase responses to these components as per the Equation 5.2.

The phase patterns for the frequency of $4.4455 \mathrm{MHz}$ is shown in the bottom right hand corner of Figure 5.12 which is similar to the ones for $5.255 \mathrm{MHz}$. Hence no change is expected in terms of the phase at such a change in frequency.

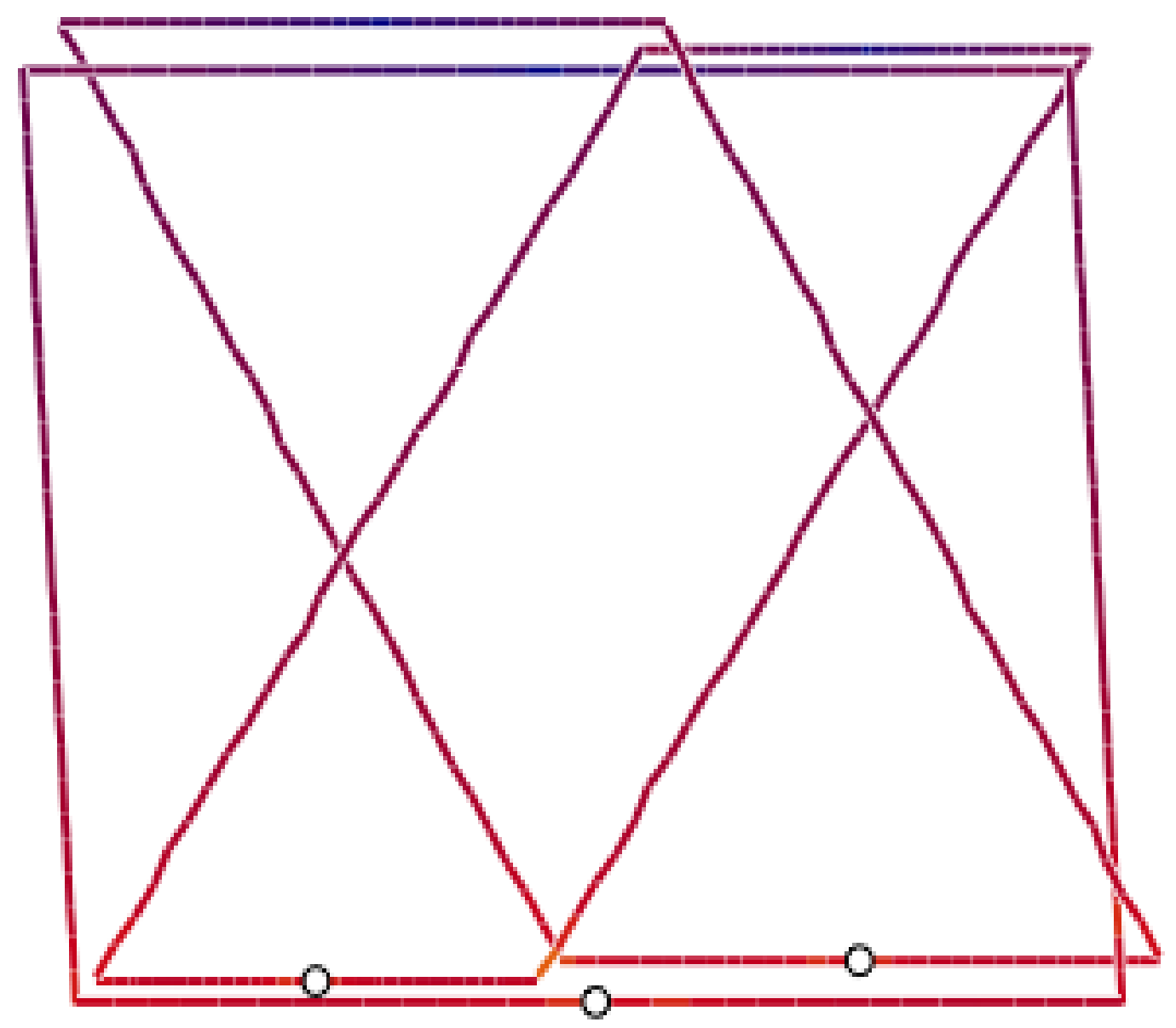

Figure 5.8 Giselle antenna model 

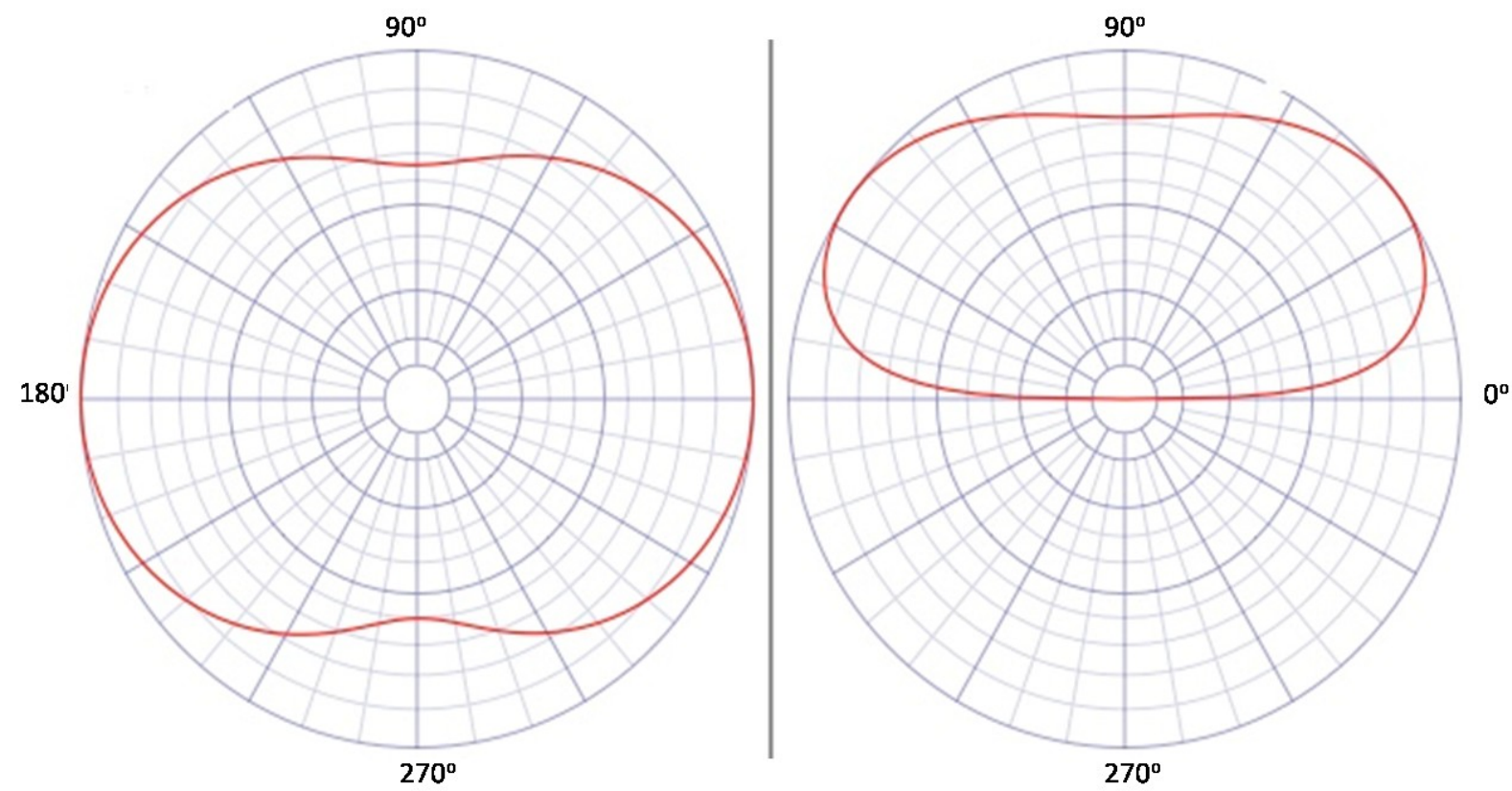

Figure 5.9 Giselle loop 1 azi muth (left) and ele vation (right)
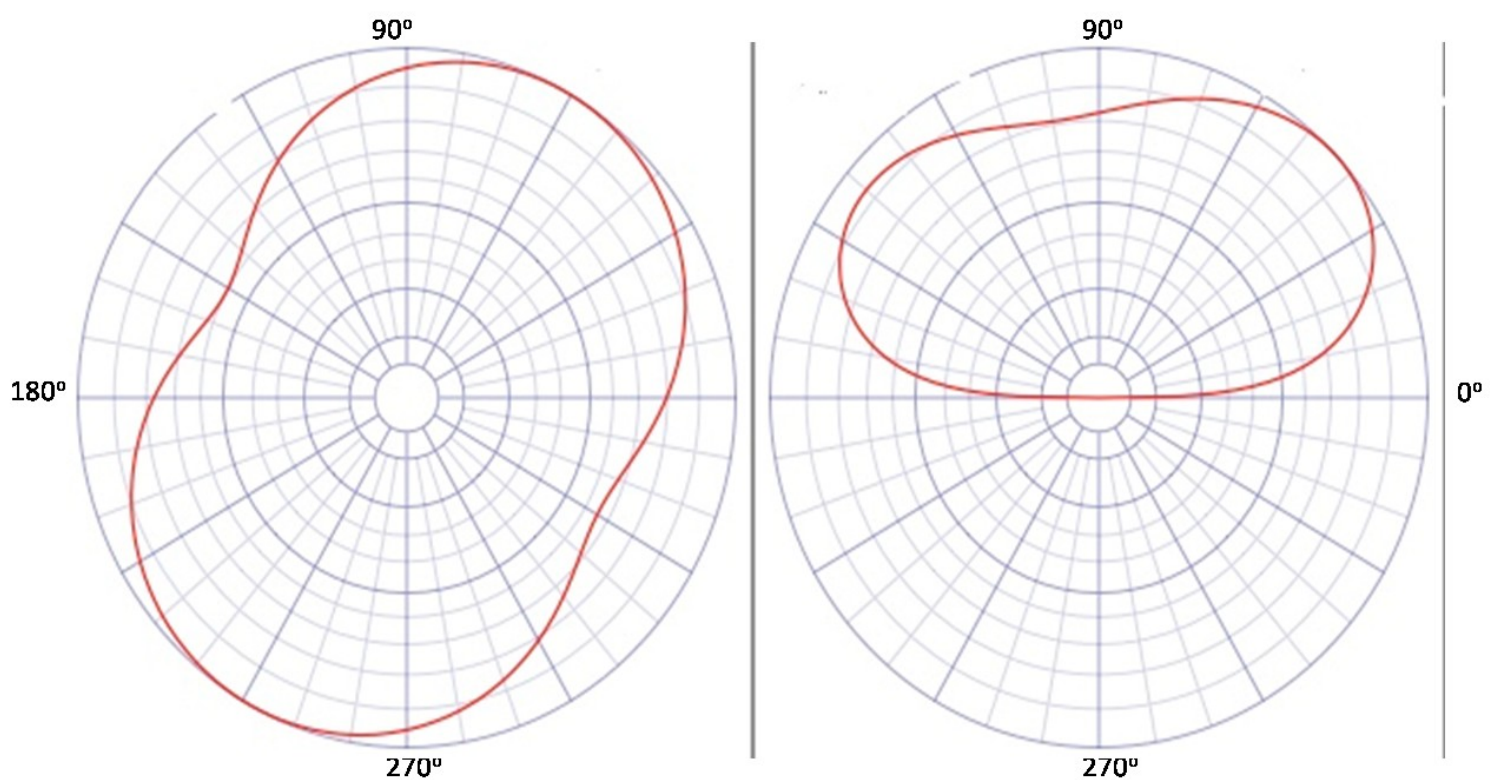

Figure 5.10 Giselle loop 2 azi muth (left) and elevation (right) plot 

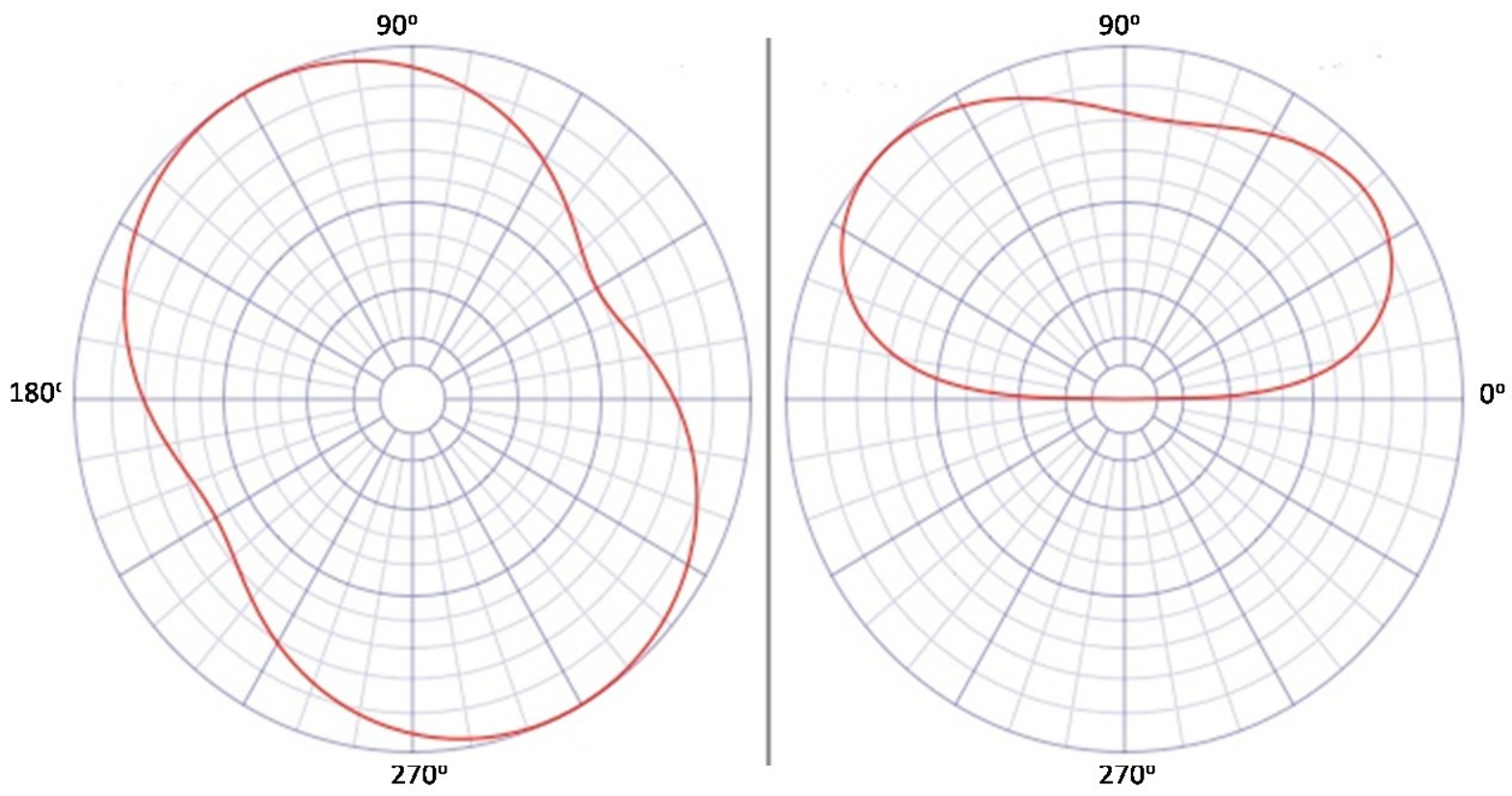

Figure 5.11 Giselle loop 3 azimuth (left) and ele vation (right) pl ot

\begin{tabular}{|l|c|c|c|}
\hline & Giselle antenna 1 & $\begin{array}{c}\text { Giselle antenna } \\
2\end{array}$ & $\begin{array}{c}\text { Giselle antenna } \\
3\end{array}$ \\
\hline Gain $(\mathrm{dBi})$ at $30^{\circ}$ & 2.40 & 1.71 & 0.69 \\
\hline Gain $(\mathrm{dBi})$ at $40^{\circ}$ & 2.40 & 2.38 & 1.11 \\
\hline Gain $(\mathrm{dBi})$ at $50^{\circ}$ & 1.89 & 2.32 & 0.78 \\
\hline Gain $(\mathrm{dBi})$ at $60^{\circ}$ & 1.83 & 1.73 & -0.02 \\
\hline Gain $(\mathrm{dBi})$ at $70^{\circ}$ & 0.03 & 0.81 & -0.09 \\
\hline
\end{tabular}

Table 5.2 Giselle antenna gain values between $30^{\circ}$ and $70^{\circ}$ elevation 

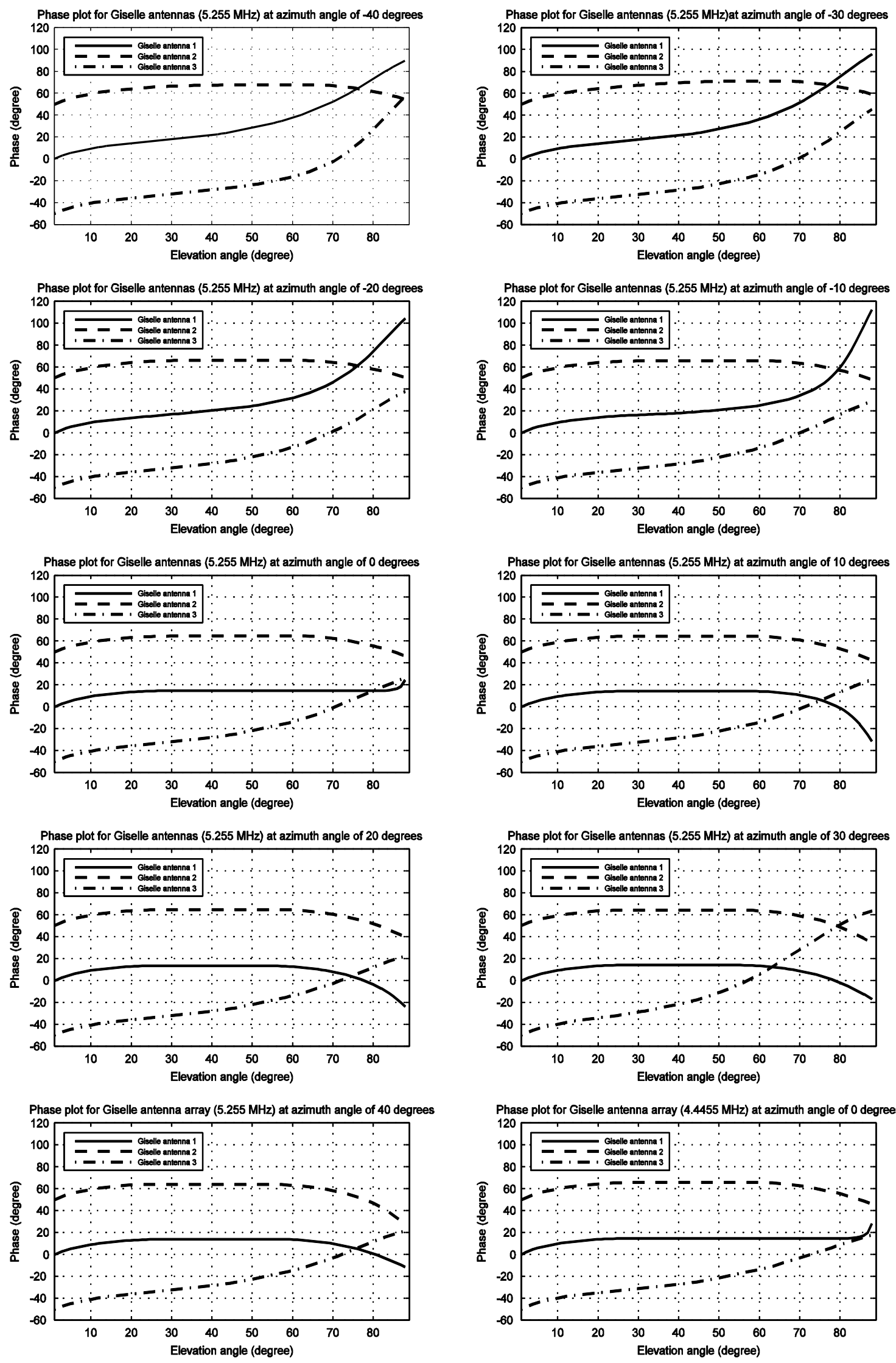

Figure 5.12 Phase patterns for Giselle antennas 


\subsubsection{XYZ collocated loops}

This antenna array was made up of 3 orthogonal loops oriented north-south, east-west and horizontal to the ground. NEC-2 model used a frequency of $5 \mathrm{MHz}$. Each wire was divided into 29 segments and a wire diameter of AWG \#14 was used which approximated the antenna wire dimensions. Average ground was used to model this antenna. The model for this antenna is shown in Figure 5.13. The sliced two dimensional radiation pattern of the north-south antenna is shown in Figure 5.14 and the 3-D pattern in Figure 5.15. The main beam of the antenna is oriented towards the direction of propagation. The main beam of the east-west oriented loop is directed away from the angle of arrival of the signal which is in the north-south direction with small azimuthal excursions as Figure 5.16 and Figure 5.17. The horizontal loop antenna has a omnidirectional pattern as shown in Figure 5.18 and Figure 5.19. This would cause the antennas to be receiving multipath components with different weighting factors applied and cause decorrelation.

The north-south and east-west loops phase patterns show very little change in the elevation angles from $0-90^{\circ}$ as seen Figure 5.20. Hence the decorrelation provided is due to the difference in amplitude pattern and the polarization diversity. The gain values for the antennas in this array are shown in Table 5.3 where considerable difference exists between the general lower gains for horizontal antenna and the other two antennas in the array. Similar antenna array has been shown to provide decorrelation on the basis of their ability to discriminate between $\mathrm{O}$ and $\mathrm{X}$ modes [Bisiaux and Bertel, 2000].

For the frequency of $4.4455 \mathrm{MHz}$ the horizontal loops phase pattern changes with a much for flat shape with the changing elevation angles. 


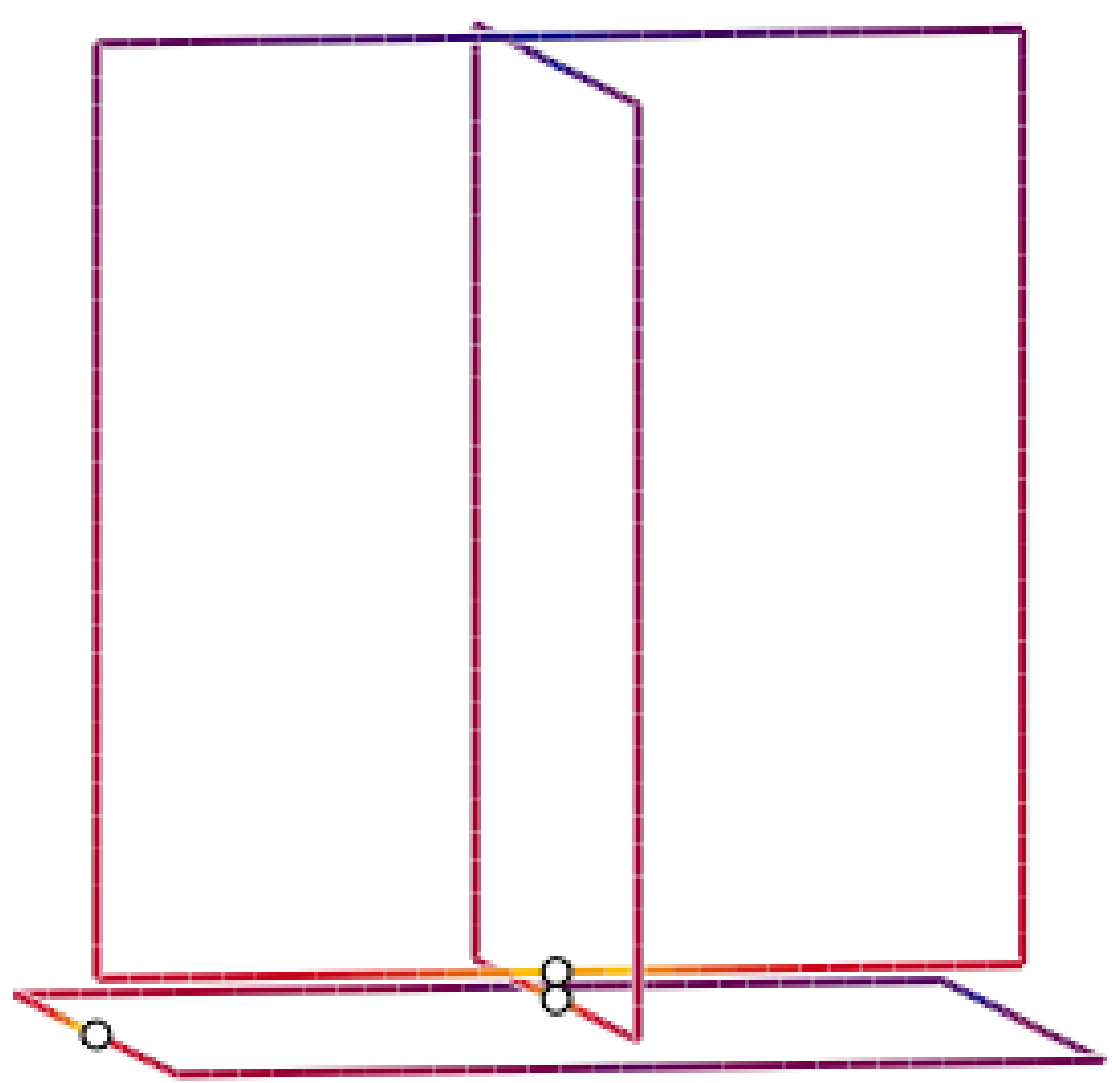

Figure 5.13 XYZ loop antenna model
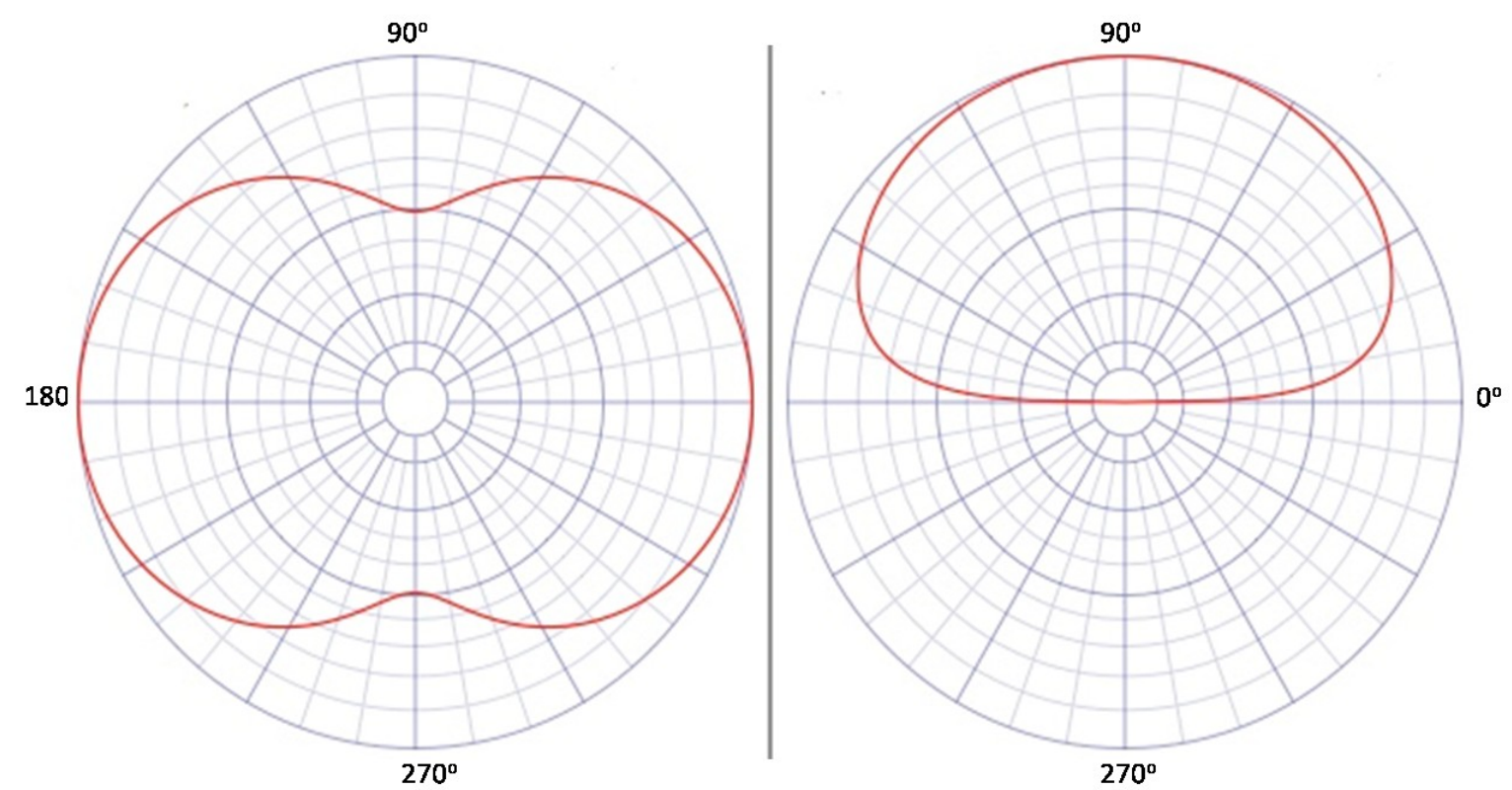

Figure 5.14 XYZ nor th-s outh loop azimuth (left) a ele vation (right) plot 


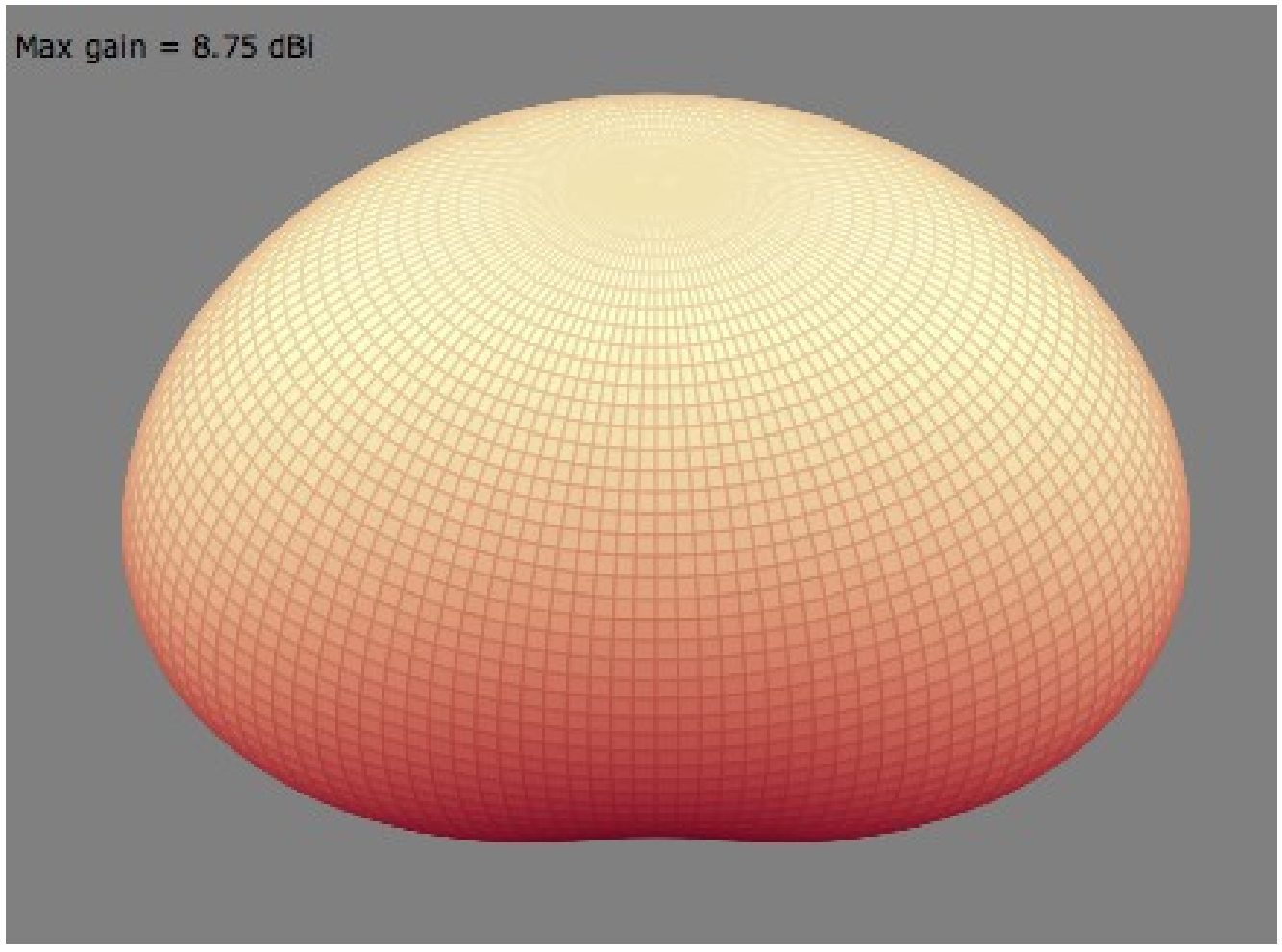

Figure 5.15 XYZ nor th-south loop 3-D radiation pattern plot
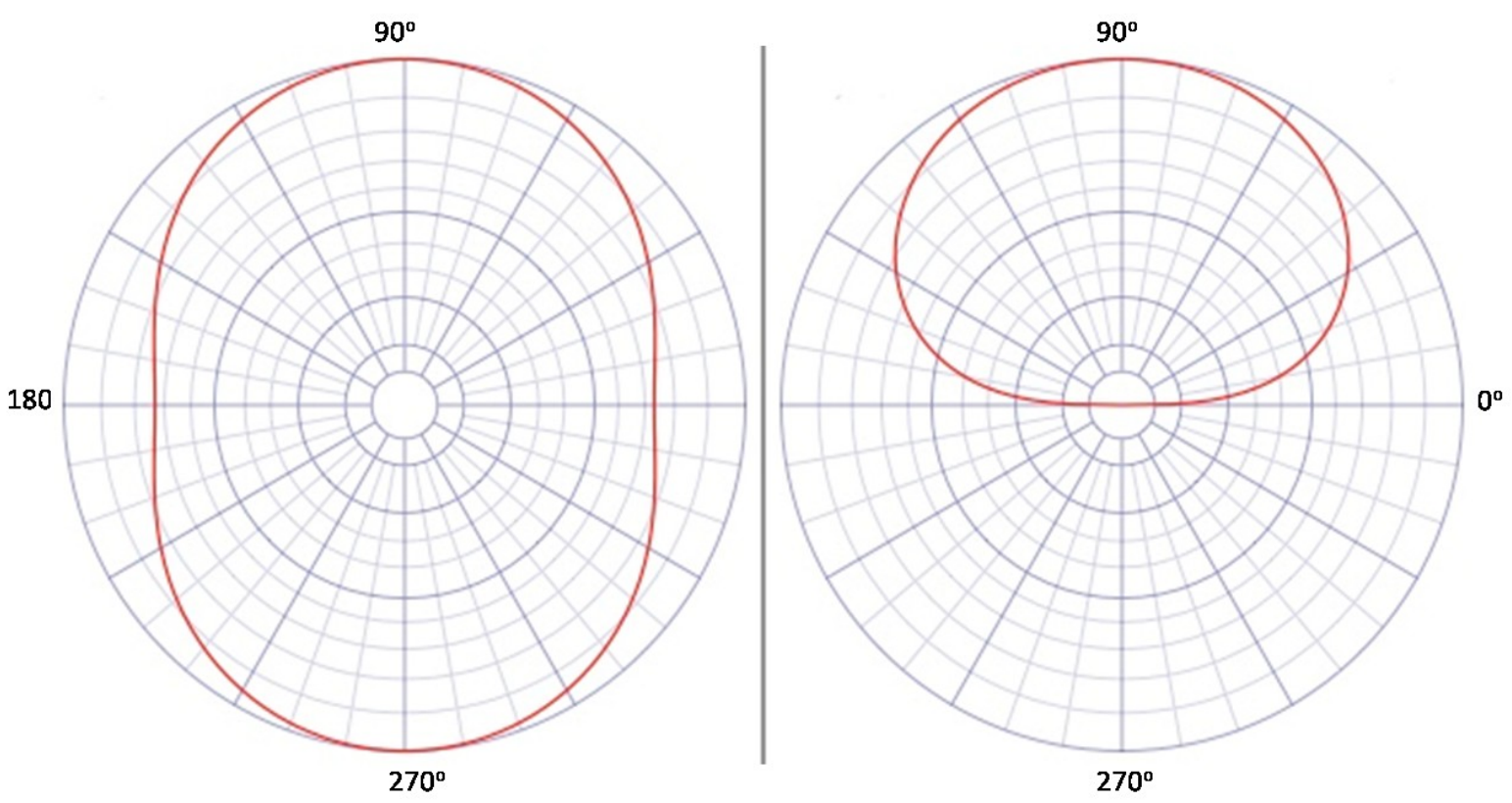

Figure 5.16 XYZ east-west loop azi muth (left) pl ot and elevation (right) pl ot 


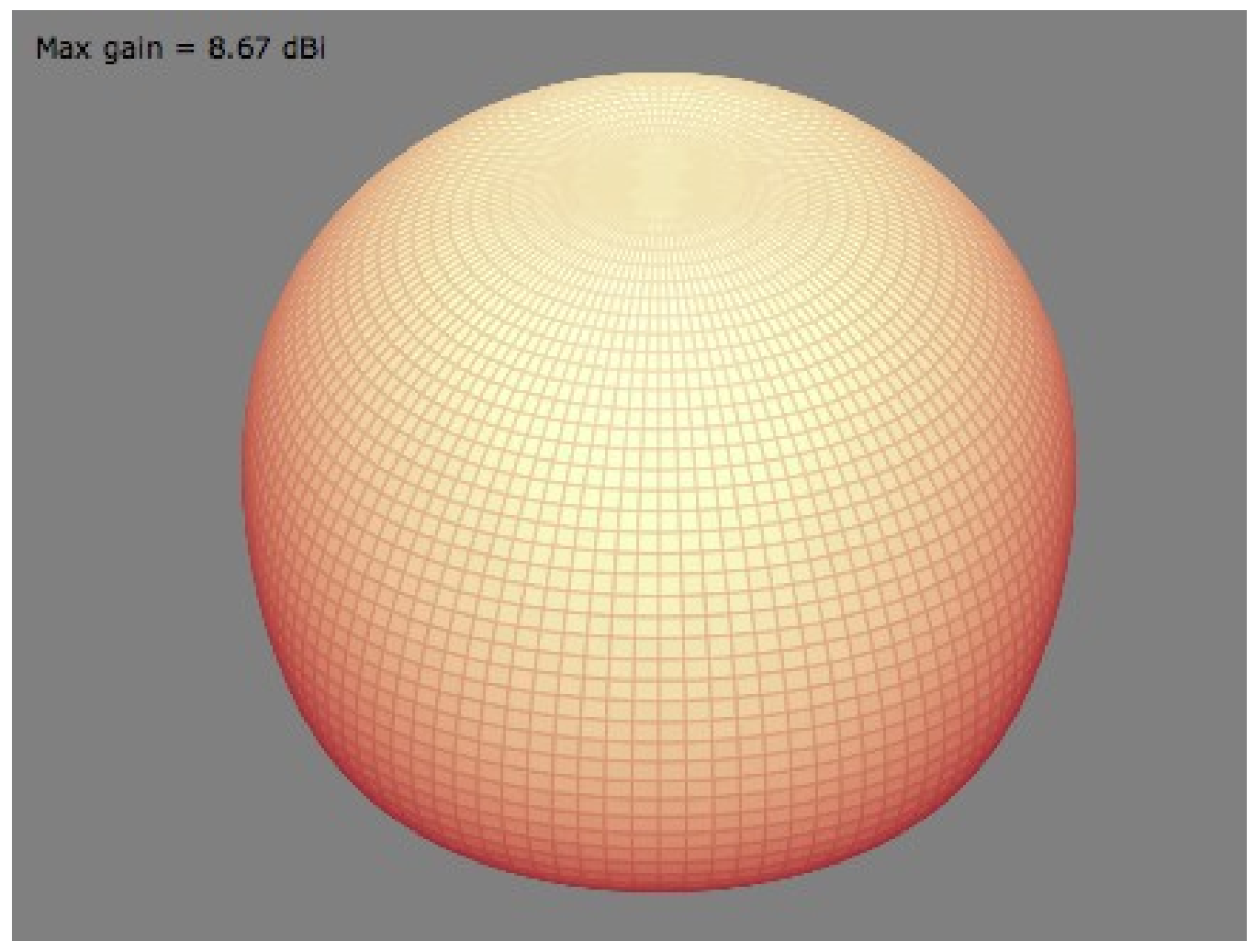

Figure 5.17 XYZ east-west loop 3-D radiation pattern pl ot
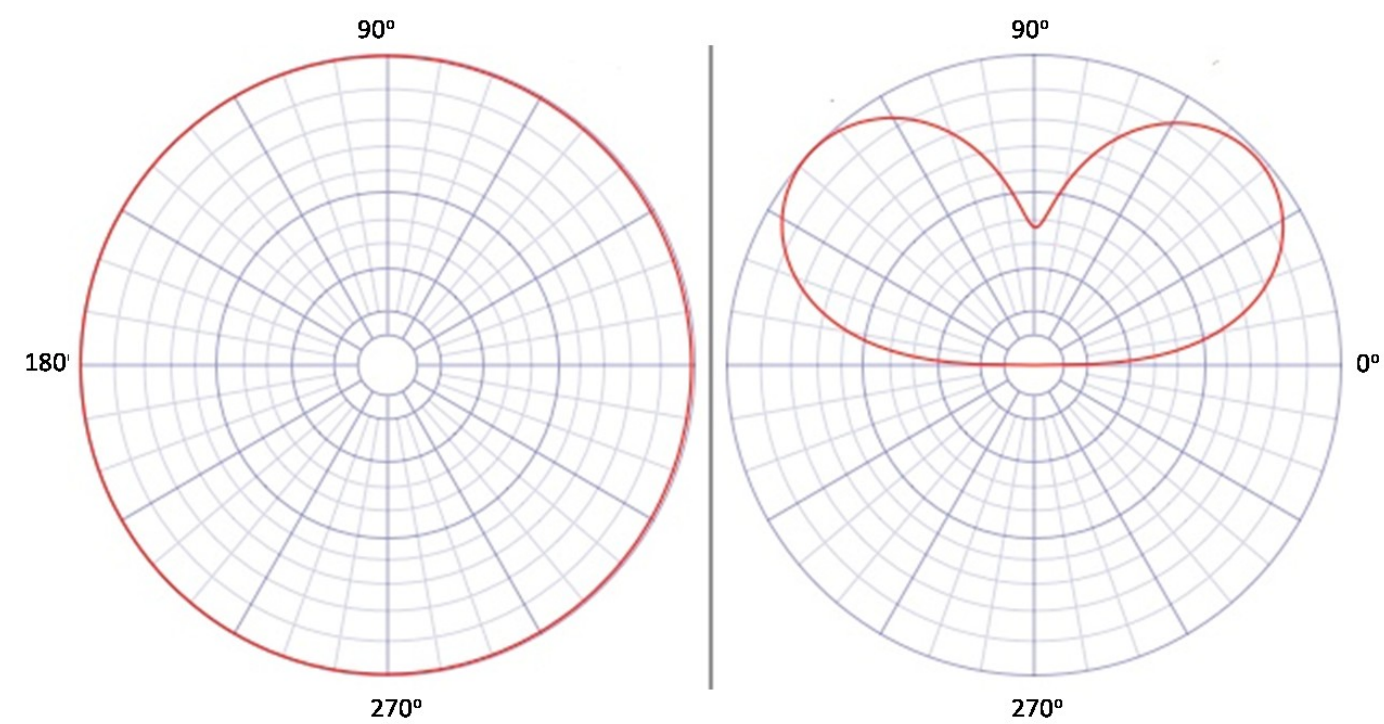

Figure 5.18 XYZ Horizontal loop azimuth (left) plot and elevation (right) 


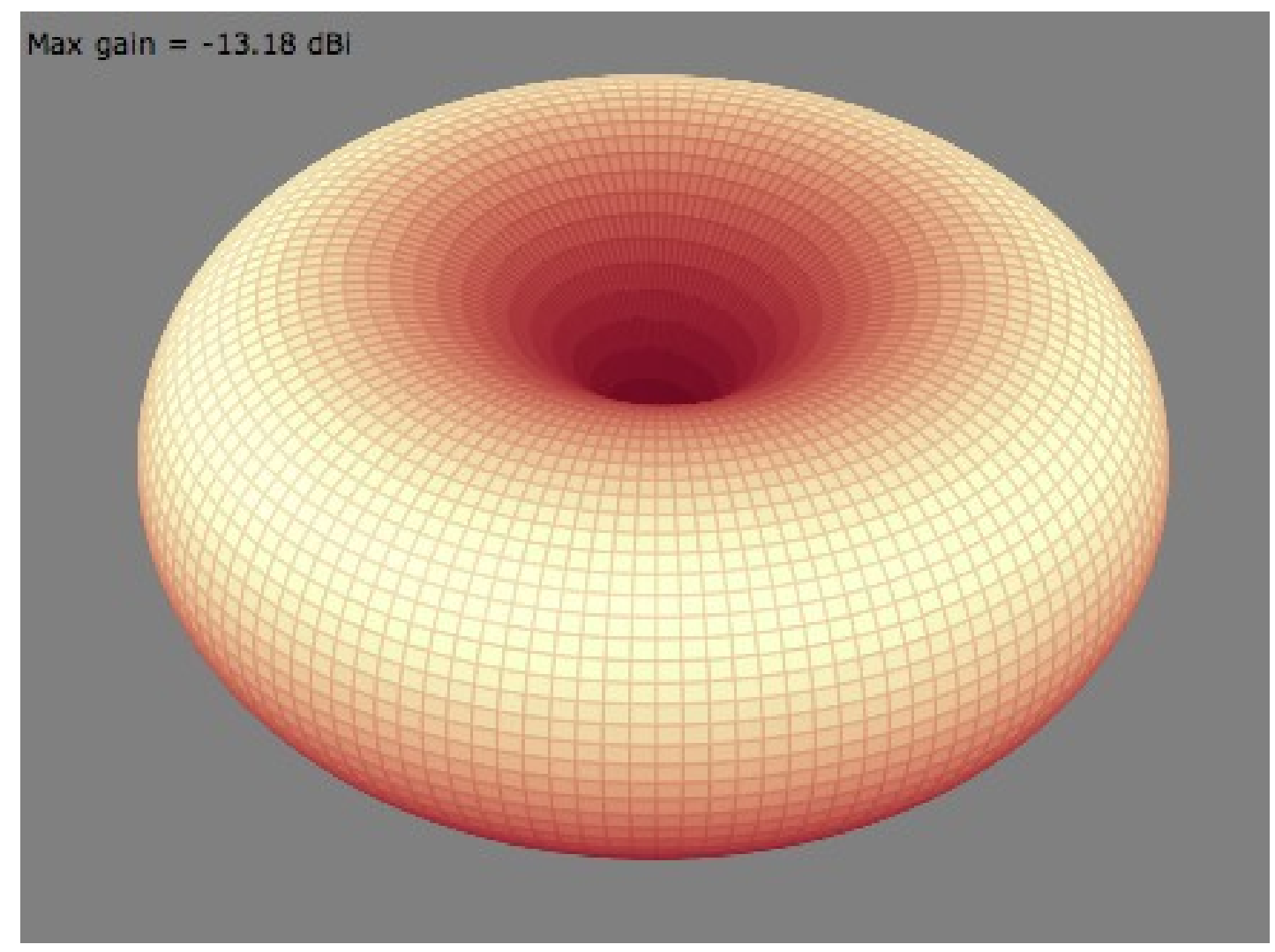

Figure 5.19 XYZ Horizontal loop 3-D radiation pattern plot

\begin{tabular}{|c|c|c|c|}
\hline & $\begin{array}{c}\text { North-south } \\
\text { loop }\end{array}$ & $\begin{array}{c}\text { East-west } \\
\text { loop }\end{array}$ & Horizontal loop \\
\hline Gain $(\mathrm{dBi})$ at $30^{\circ}$ & 6.96 & 4.06 & -14.32 \\
\hline Gain $(\mathrm{dBi})$ at $40^{\circ}$ & 7.68 & 5.88 & -13.47 \\
\hline Gain $(\mathrm{dBi})$ at $50^{\circ}$ & 8.14 & 7.06 & -13.78 \\
\hline Gain $(\mathrm{dBi})$ at $60^{\circ}$ & 8.43 & 7.84 & -14.97 \\
\hline Gain $(\mathrm{dBi})$ at $70^{\circ}$ & 8.61 & 8.32 & -17.56 \\
\hline
\end{tabular}

Table 5.3 XYZ antenna gain values between $30^{\circ}$ and $70^{\circ}$ at $0^{\circ}$ azi muth 

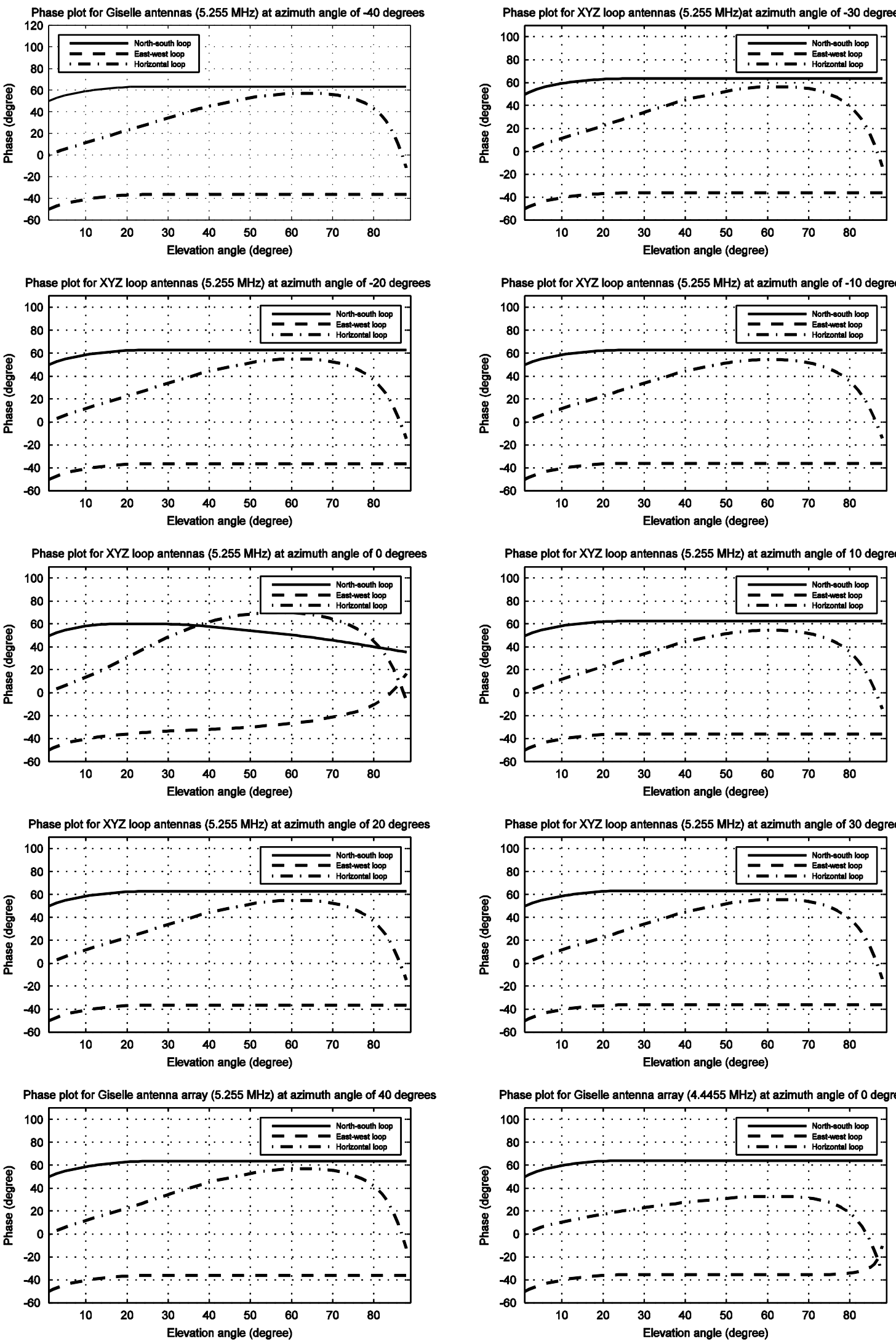

Phase plot for Giselle antenna array $(4.4455 \mathrm{MHz})$ at azimuth angle of 0 degrees

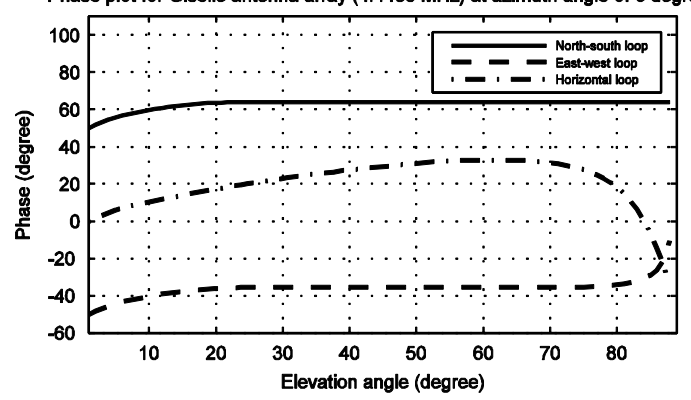

Figure 5.20 Phase patterns for XYZ antennas 


\subsubsection{Modelling results for crossed dipoles with a vertical antenna}

This antenna array consisted of two crossed dipole antennas and a vertical monopole antenna on top of the centre of the two horizontal dipole antennas and thus is an example of collocated heterogeneous arrays. The NEC-2 model for this array was built using the details given in Chapter 3 for a frequency of $5 \mathrm{MHz}$. Each wire was divided into 29 segments and a wire diameter of AWG \#14 was used which approximated the antenna wire dimensions. Average ground was used to model this antenna. The model for this antenna is shown in Figure 5.21.

The sliced two dimensional radiation pattern of the north-south antenna is shown in Figure 5.22 and the 3-D pattern in Figure 5.23. The antenna has its radiation pattern pointing towards high elevation angles and the maximum energy away from the direction of arrival of the incoming wave. The east-west oriented dipole also has the same pattern in elevation as expected but the azimuth pattern is directing energy in the direction of the incoming signal from Durham. Thus is shown in Figure 5.24 and Figure 5.25. The radiation pattern of the vertical monopole is directed towards low elevation angles and is omni-directional (Figure 5.26 Figure 5.27). Thus it would be receiving the multipath signals from the E layer of the ionosphere as opposed to the north-south and east-west dipoles which would be receiving multipath components from the $\mathrm{F}$ layers. The phase variations of the three antennas in this array are plotted in Figure 5.28.

The north-south and east-west dipoles have approximately same slopes except for the azimuth angle of zero while the vertical monopole antenna has a different slope. The slopes of all the antennas are however positive. Table 5.4 depicts the gains variations for the Giselle loops between $30^{\circ}$ and $70^{\circ}$. 


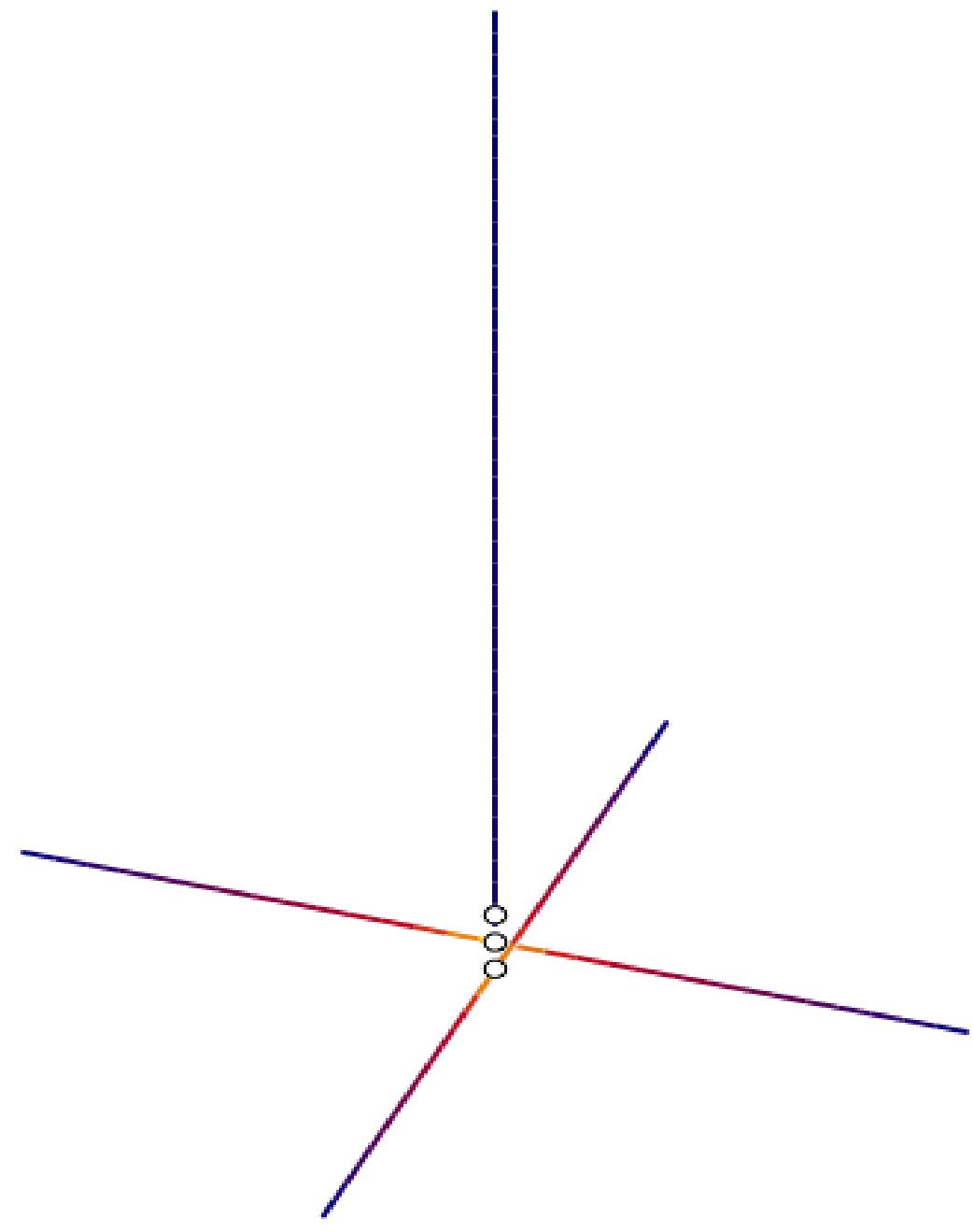

Figure 5.21 Crossed dipoles with a monopole NEC-2 model
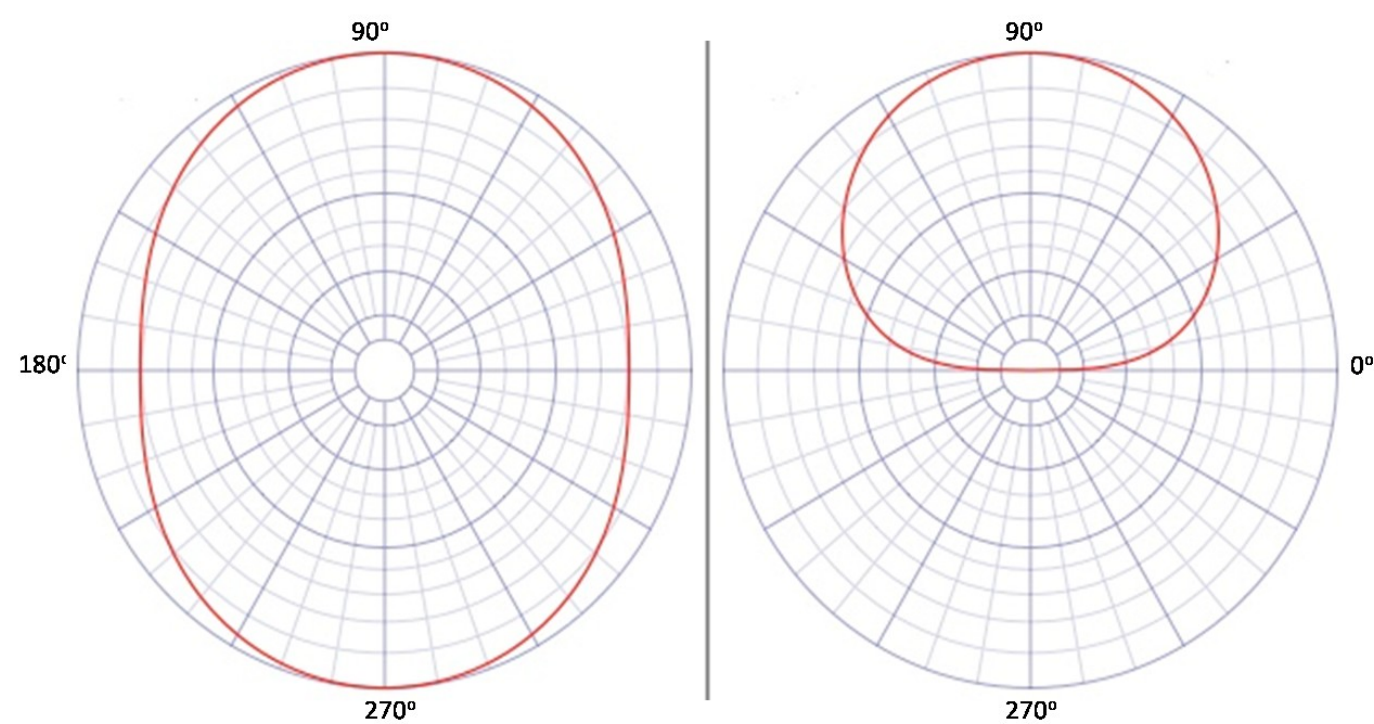

Figure 5.22 North-south dipole azimuth (left) and ele vation (right) plot 


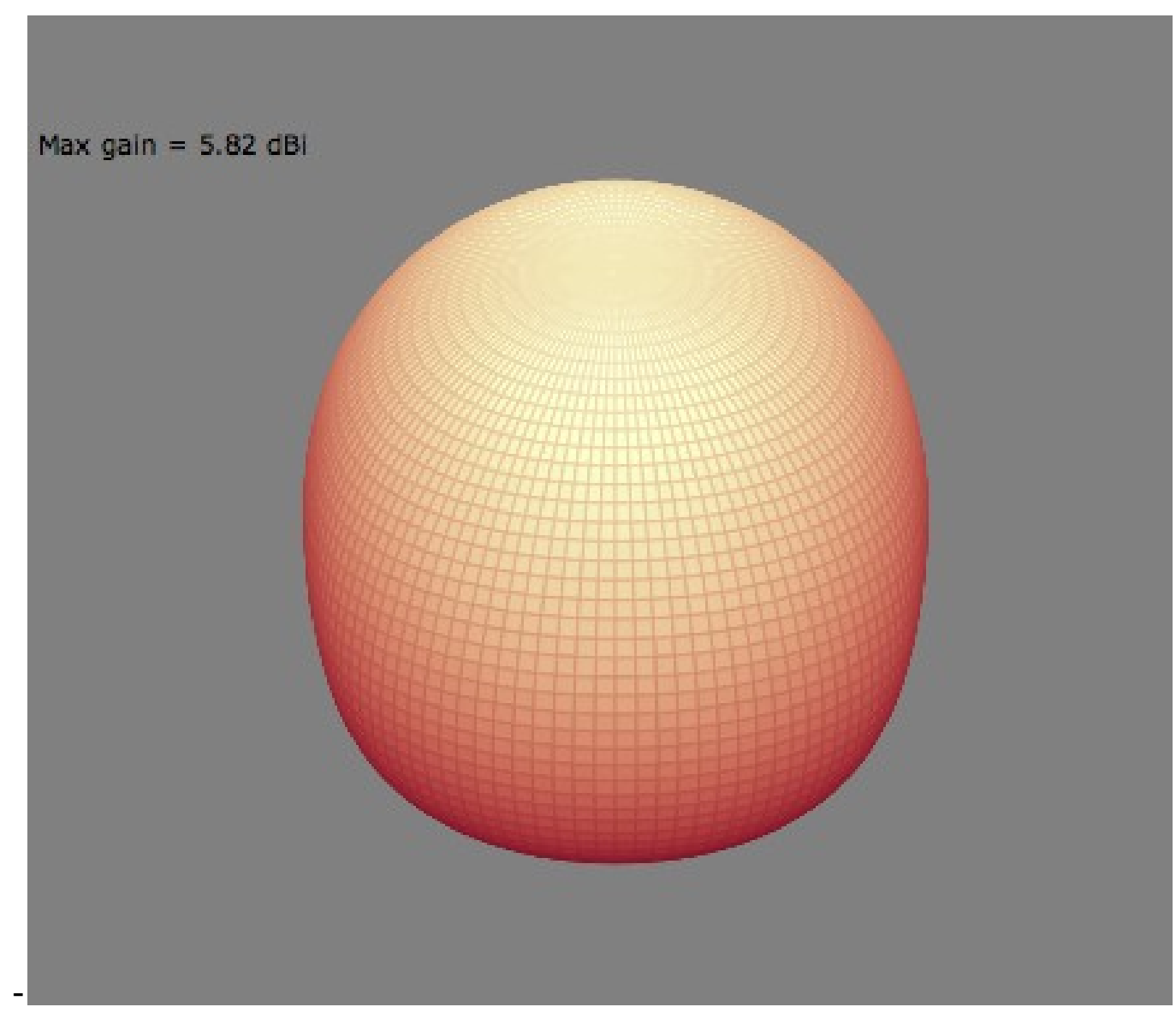

Figure 5.23 North-south dipole 3-D radiation pattern plot
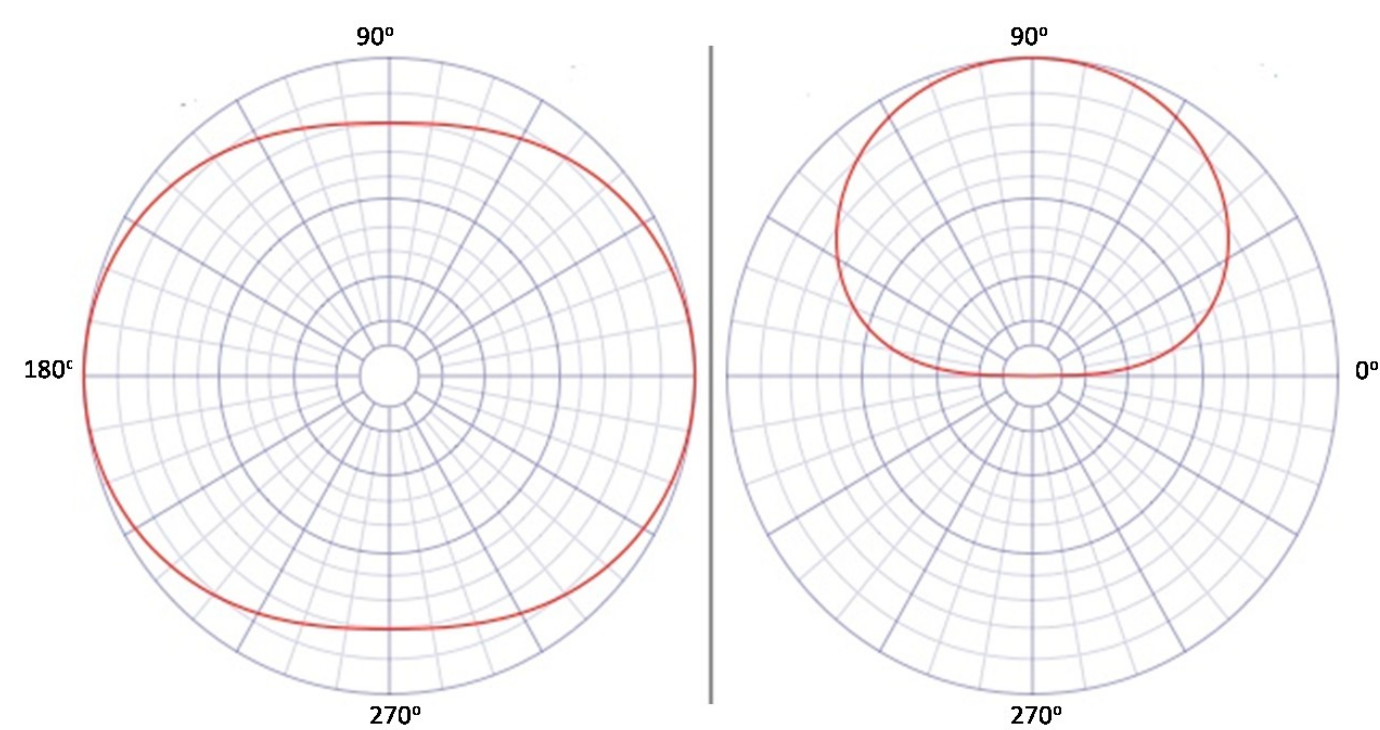

Figure 5.24 East-west dipole azi muth (left) and elevation (right) plot 


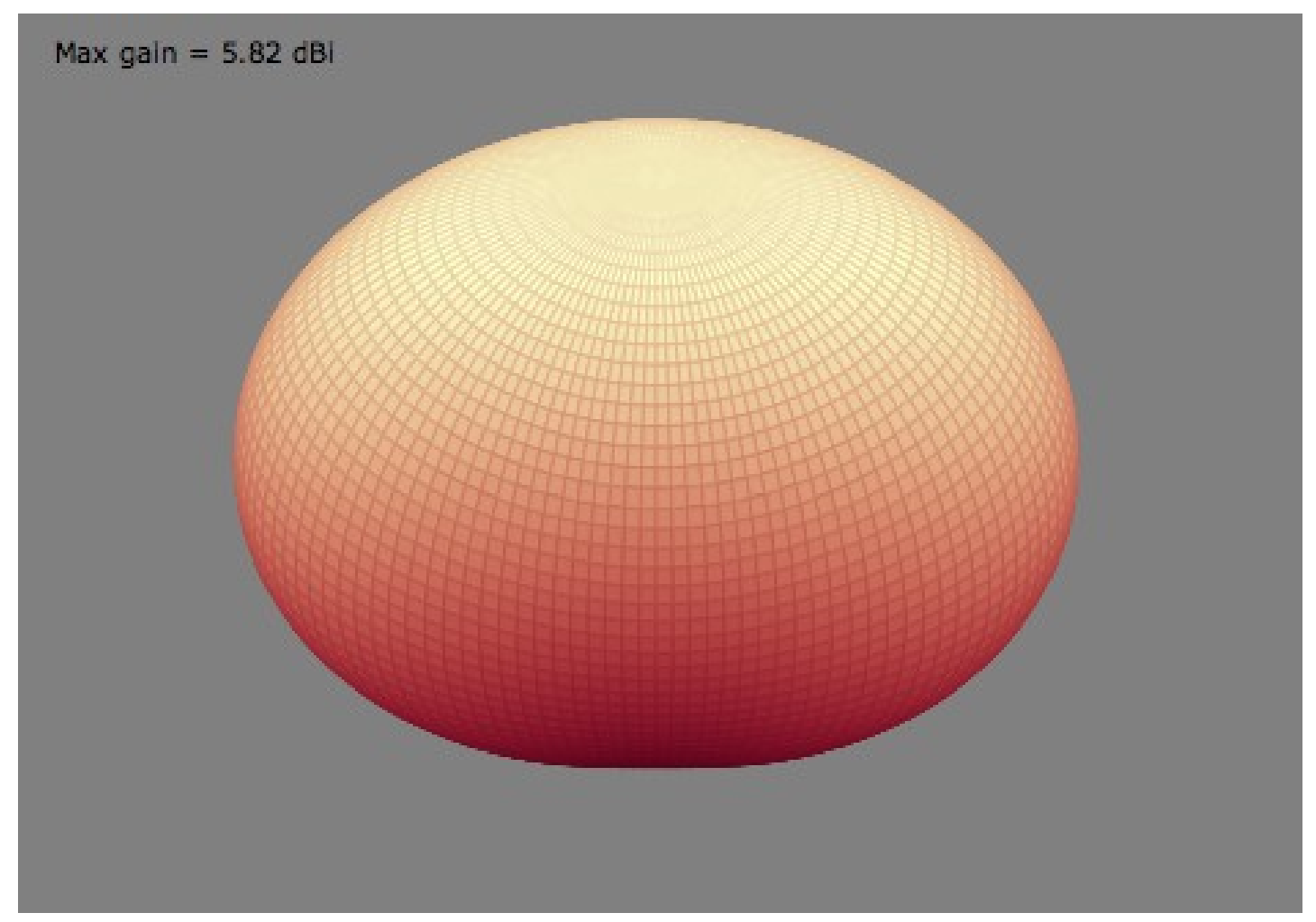

Figure 5.25 East-west dipole 3-D radiation pattern pl ot
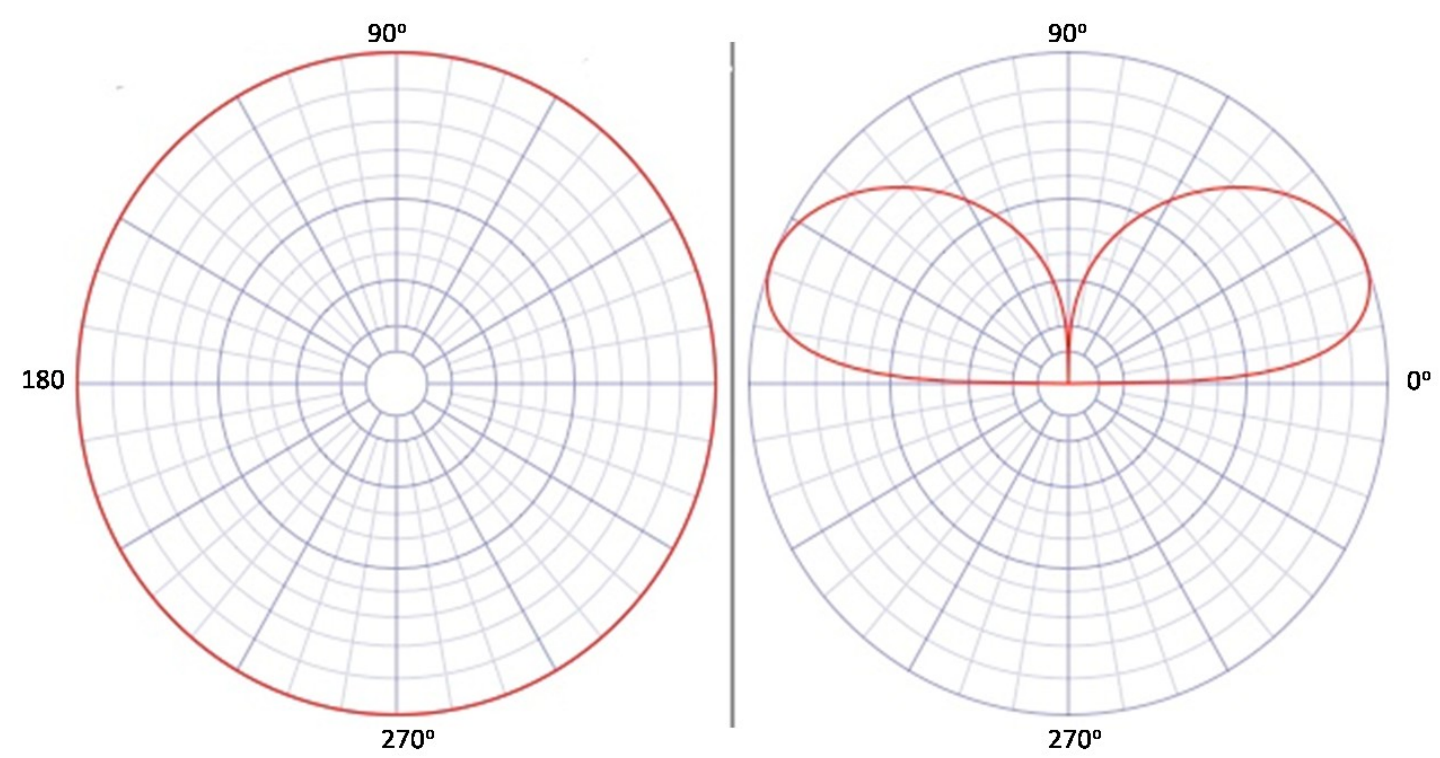

Figure 5.26 Vertical monopole azimuth (left) and ele vation (right) plot 


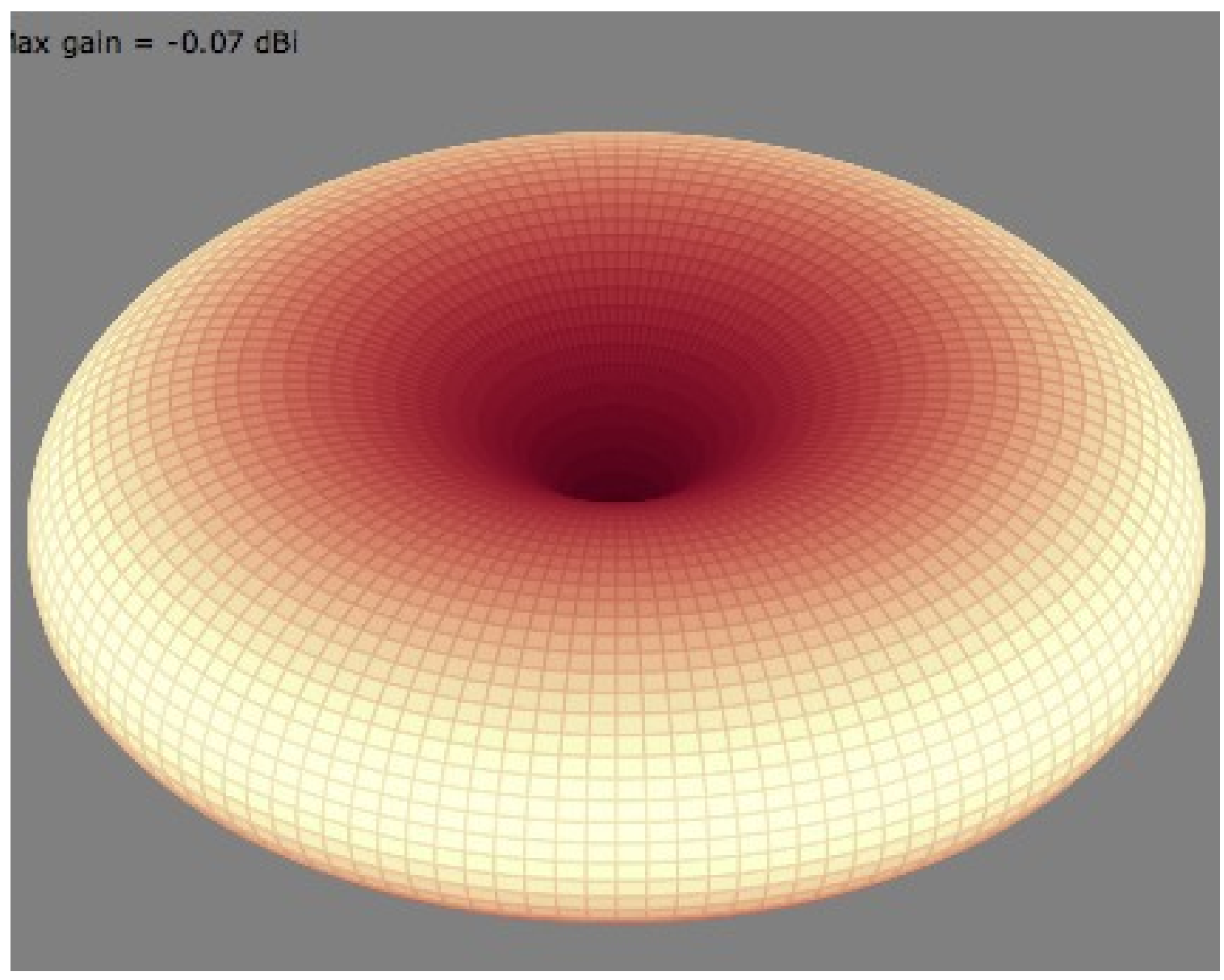

Figure 5.27 Vertical monopole 3-D radiation pattern plot

\begin{tabular}{|l|c|c|c|}
\hline & North-south dipole & East-west dipole & Vertical monopole \\
\hline Gain $(\mathrm{dBi})$ at $30^{\circ}$ & -7.56 & -6.36 & -1.63 \\
\hline Gain $(\mathrm{dBi})$ at $40^{\circ}$ & -5.82 & -4.37 & -2.15 \\
\hline Gain $(\mathrm{dBi})$ at $50^{\circ}$ & -4.28 & -3.04 & -3.37 \\
\hline Gain $(\mathrm{dBi})$ at $60^{\circ}$ & -3.00 & -2.14 & -5.35 \\
\hline Gain $(\mathrm{dBi})$ at $70^{\circ}$ & -2.05 & -1.55 & -8.45 \\
\hline
\end{tabular}

Table 5.4 Dipole array gain values between $30^{\circ}$ and $70^{\circ}$ elevation at $0^{\circ}$ azi miuth 

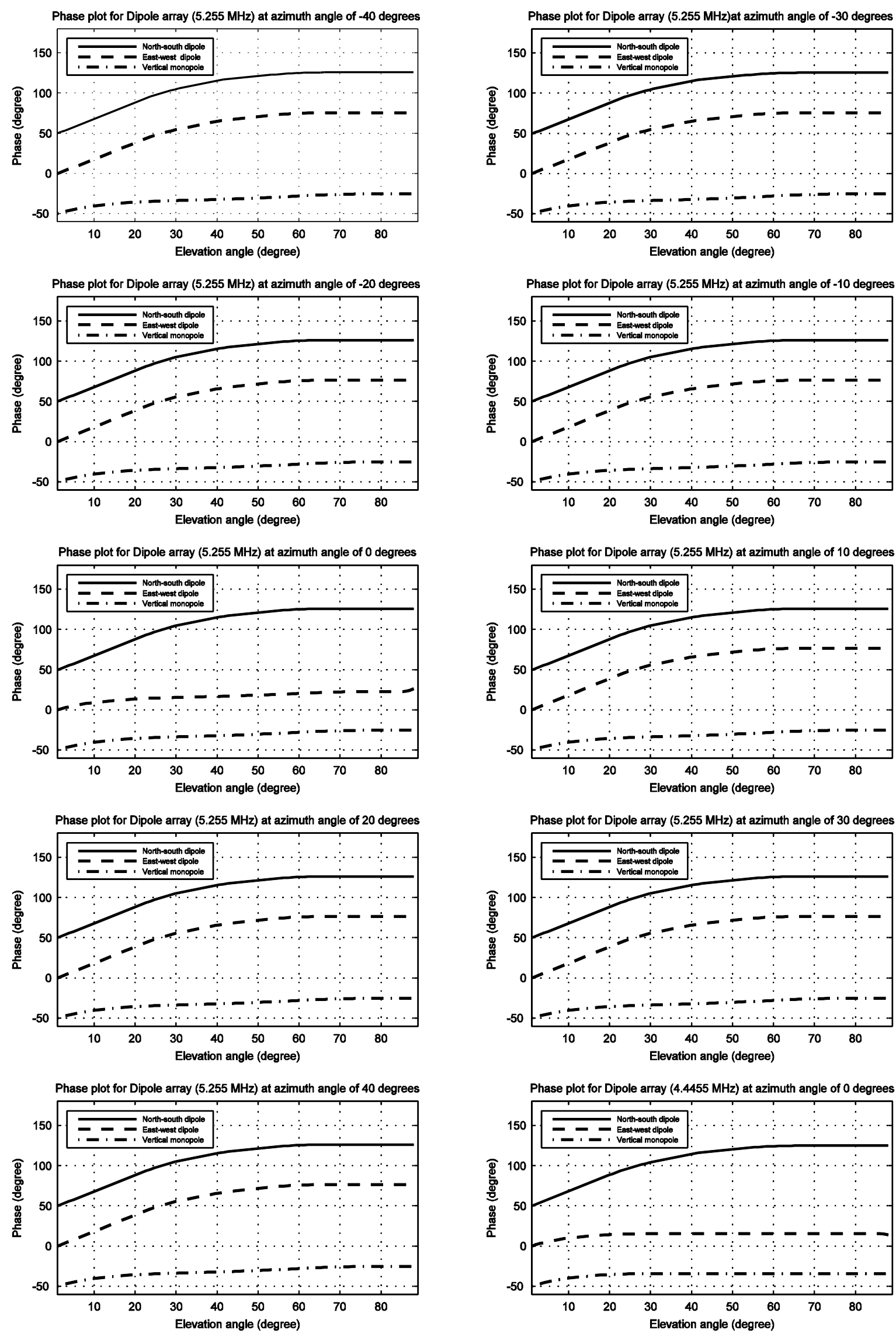

Figure 5.28 Phase patterns for dipole array 


\subsection{Concluding Remarks}

The modelling results indicate that the difference in radiation patterns of the antennas in a collocated array in terms of both amplitude and phase can contribute to the decorrelation measured. Antennas could be modelled considering only the parameter of phase pattern which could be suitable for HF-MIMO deployment. The crossed wire inverted $\mathrm{V}$ antennas are an ideal example of this case where antennas are giving decorrelated results based on the differential phase change. The Giselle loop antennas show both the pattern diversity as well as phase differences and a combination of the both introduces decorrelation in the received signals. The XYZ loop arrays show pattern diversity especially the horizontal loop with the other two loops. North-south and east-west loops have almost identical phase pattern slopes which indicate that the decorrelation between these two antennas is not due to the phase patterns. The decorrelation between these two antennas found in experiments will be due to the magnitude pattern diversity and the polarization diversity [Bisiaux and Bertel, 2000]. The crossed dipoles show similar elevation pattern but the azimuthal variations are shifted by $90^{\circ}$. The phase patterns of these two antennas have similar slopes as well. Hence the polarization diversity may be causing the decorrelation in the signals received by these antennas [Peco et al., 2009]. The vertical monopole however exhibits pattern diversity with the other two antennas in the array. 


\section{Conclusion and future work}

This experimental study was based on the application of MIMO techniques in the HF band. The focus of the project was to study the decorrelation provided by the ionosphere for different arrangements of antenna arrays. Uncoded continuous wave signals were used in all campaigns. The antenna arrays ranged from the traditional spaced arrays to some novel large and small collocated ones utilizing electrically small elemenst. Antenna modelling techniques were used to identify factors that could lead to the design of antenna arrays suitable for HF-MIMO.

The correlation and capacity estimates were calculated for the different types of antennas in the arrays from data collected on a campaign basis over a span of 2 years. The capacity estimates were consistent with the correlation results and showed an increase in capacity with increasing number of antennas. Also the antennas pairs with lowest correlation tended to give the highest values for the capacity estimates. Initial experiments used traditional homogenous spaced arrays. The assertion in literature that the correlation between the antennas in a homogenous spaced array depends on the distance that separates them was confirmed. Also it was found out that even a separation distance of $\lambda / 2$ was not sufficient to introduce sufficient decorrelation under the prevailing ionospheric conditions. In the HF band this translates into distances in the range of 10's of metres and going higher in the lower part of the HF spectrum. Such large area requirements cannot be met in many applications such as aeroplanes and ships. Also the deployment of antennas requires more time and effort. Though there has been research on using closely spaced antennas with decorrelation networks [Dumanli et al., 2007] to achieve decorrelation, it is still not mature and the drawbacks of separate antenna placements still remains. In addition the varying response of the antennas towards multipath was dependent on the orientation of the array. This limits 
their usage in application of such arrays in ships, aeroplanes etc where the orientation will change unpredictably causing the performance of the system to fluctuate.

The experiments with the large collocated antennas followed next with the crossed wire inverted $\mathrm{V}$ wires antennas orientated in the north-south and east-west direction. These provided good decorrelation results consistently over a variety of ionospheric conditions. This included experiments at different hours of the day over different parts of the year. Such results were attributed to the difference in the phase patterns of these two antennas which gave an insight into a dimension normally overlooked in the research undertaken on the decorrelation of the signals at MIMO antennas. Thus antenna arrays could be modelled specifically for HF-MIMO applications in mind that could take maximum advantage of the multipath effects of the channel. After the successful use of large collocated antennas, experiments were conducted using electrically small antennas built at Durham University. The first of these to be tested was the so called XYZ loop array consisting of the north-south, eastwest and horizontal (to the ground) loop antenna's. It gave good decorrelation results comparable to the crossed wire antennas under similar conditions. Antenna modelling of this array showed that the north-south and east-west antenna had a negligible phase difference. However they showed some pattern diversity which is likely to contribute to the decorrelation. The horizontal loop paired with the other two loops in the arrays respectively will provide a combination of both pattern and phase diversity. In addition the antenna response to the $\mathrm{O}$ and the $\mathrm{X}$ modes may be different resulting in further reduction of the correlation coefficients. The Giselle antennas also showed good decorrelation results. The modelling of Giselle revealed that although antenna 1 and antenna 2 had small phase differences, antenna pairs 1 and 3, and 2 and 3 did have phase differences. The Giselle antenna also showed pattern diversity. The crossed wire 
dipoles used in experiments also received de-correlated signals however the modelling of this arrays showed no phase pattern differences between the two dipoles. The two dipoles however showed the phase differences when paired with the vertical monopole. There was however no phase pattern changes with the azimuth angle and the phase patterns remained essentially the same. The crossed dipoles showed polarization diversity which could be the reason for the decorrelation provided. The modelling results for crossed wire inverted $\mathrm{V}$ antennas were compared with carefully selected data from the experiments where the signal reflected from different ionospheric layers. This was found out to be consistent with the modelling results whereby a large phase difference would cause a higher decorrelation between the antenna pairs.

The modelling of the collocated antennas and their use in the experiments showed that MIMO techniques could be utilized in the HF band. The selection of antennas and their orientations in space are however of prime importance and antenna modelling software's could be used to design antennas taking into consideration their polarization and pattern (both amplitude and phase) diversity.

In the future this work can be extended to model more collocated antennas based on the principles outlined in this thesis. Specifically antennas with phase patterns having inverted slopes with maximum difference could be modelled. To help in such modelling, software could be developed which uses the NEC2 engine to optimize parameters of the antennas for divergent phase patterns.

Experiments over other paths could be conducted which consist of longer and shorter paths. This would further help in isolating the modes of transmission and the effect of the antenna patterns on these modes. 
Transmissions with MIMO coding could be introduced which would allow for the transmission of digital information and BER to be measured. This was unsuccessfully tried in some of the campaigns but could be pursued in future and experiments conducted with really voice/image/video data. 


\section{REFERENCES}

Alamouti, S. (1998), A simple transmitter diversity technique for wireless communications, IEEE Journal on Selected Areas in Communications, Special issue on Signal Processing for Wireless Communications, 16(8), 1451-1458.

Andrews, M. R., P. P. Mitra, and R. deCarvalho (2001), Tripling the capacity of wireless communications using electromagnetic polarization, Nature, 316-318.

Balanis, C. (1982), Antenna theory : analysis and design, Harper \& Row, New York.

Bertel, L., D. Lemur (2002), Antenna effects on HF systems, URSI, $27^{\text {th }}$ General Assembly of the International Union of Radio Science, Maastricht, Netherlands.

Bisiaux, A., L. Bertel (2000), Antenna diversity as a means of improving HF transmissions, paper presented at Proceedings of the IEE HF Radio Systems and Techniques, Guildford, UK.

Bohnke, R., V. Kuhn, and K. D. Kammeyer (2004), Multistratum space-time codes for the asynchronous uplink of MIMO-CDMA systems, paper presented at IEEE Eighth International Symposium on Spread Spectrum Techniques and Applications, Sydney, Australia.

Bonek, E., M. Steinbaner (2001), Double-directional channel measurements, paper presented at Eleventh International Conference on Antennas and Propagation, Manchester, UK. 
Brine, N. L., C. C. Lim, and A. D. Massie (2006), Capacity estimation for HF-MIMO channel, paper presented at Proceedings of the Sixth Symposium on Radiolocation and Direction Finding, , Southwest Research Institute, San Antonio, Texas, US A.

Bubenik, D. (1977), A practical method for the numerical evaluation of Sommerfeld integrals, IEEE Transactions on Antennas and Propagation, 25(6), 904-906.

Cebik, L. B. (2001), A beginner's guide to modelling with NEC QST, 34-38.

Chen, K., 18 August 2011: cocoaNEC 2.0. [Available online at http://www.w7 ay.net/site/Applications/cocoaNEC/].

Chizhik, D., G. J. Foschini, M. J. Gans, and R. A. Valenzuela (2002), Keyholes, correlations, and capacities of multielement transmit and receive antennas, IEEE Transactions on Wireless Communications, 1(2), 361-368.

Chizhik, D., J. Ling, P. W. Wolniansky, R. A. Valenzuela, N. Costa, and K. Huber (2003), Multiple-input-multiple-output measurements and modeling in Manhattan, IEEE Journal on Selected Areas in Communications, 21(3), 321-331.

Chuah, C. N., G. J. Foschini, R. A. Valenzuela, D. Chizhik, J. Ling, and J. M. Kahn (2000), Capacity growth of multi-element arrays in indoor and outdoor wireless channels, paper presented at IEEE Wireless Communications and Networking Conference, WCNC, Chicago, USA.

Crampton, C. (1947), Naval radio direction-findin - Part IIIA: Radiocommunication, Journal of the Institution of Electrical Engineers, 94(11), 132-153. 
Davies, K. (1990), Ionospheric Radio, Peregrinus on behalf of the Institution of Electrical Engineers, London, U.K.

Dietrich, C. B., K. Dietze, J. R. Nealy, and W. L. e. Stutzman (2001), Spatial, polarization and pattern diversity for wireless handheld terminals. IEEE Transactions on Antennas and Propagation, 49(9), 1271-1281.

Dumanli, S., C. J. Railton, and D. L. Paul (2007), Decorrelation of a Closely Spaced Antenna Array and Its Influence on MIMO Channel Capacity, paper presented at The Second European Conference on Antennas and Propagation, EUCAP, Edinburgh, U.K.

Eiceg, V., H. Sampath, and S. Catreux-Erceg (2006), Dual-polarization versus singlepolarization MIMO channel measurement results and modeling, IEEE Transactions on Wireless Communications, 5(1), 28-33.

Erhel, Y., L. Bertel, and F. Marie (1998), A method of direction finding operating on an array of collocated antennas, paper presented at IEEE APS/URSI International symposium on Antennas and Propagation, Atlanta, USA.

Erhel, Y., D. Lemur, and L. Bertel (1997), HF radio direction finding operating on an heterogeneous array of antennas, paper presented at Tenth International Conference on Antennas and Propagation, Edinburgh, U.K.

Feeney, S. M., S. Salous, E. M. Warrington, S. D. Gunashekar, N. Abbasi, L. Bertel, D. Lemur, and M. Oger (2009), Compact antenna arrays for HF MIMO applications, paper presented at 11 th International Conference on Ionospheric radio Systems and Techniques, The Institution of Engineering and Technology, Edinburgh, UK. 
Foschini, G. J. (1996), Layered space-time architecture for wireless commmunication in a fading environment when using multi-element antennas, Bell Labs Technical Journal, 1(2), 41-59.

Foschini, G. J., M. J. Gans (1998), On limits of wireless communications in a fading environment when using multiple antennas, Wireless Personal Communications, 6(3), 311-335.

Frazer, G. J., T. J. Harris (2006), Implications of O-X mode interference on large HF receive arrays, WARS 2006, Leura, Australia.

Gesbert, D., H. Bolcskei, D. A. Gore, and A. Paulraj (2002), Outdoor MIMO wireless channels: models and performance prediction IEEE Transactions on Communications, 50(12), 1926-1934.

Golden, G. D., C. J. Foschini, R. A. Valenzuela, and P. W. Wolniansky (1999), Detection algorithm and initial laboratory results using V-BLAST space-time communication architecture, Electronics Letters, 35(1), 14-16.

Gunashekar, S. D., E. M. Warrington, S. Salous, W. Kassem, L. Bertel, D. Lemur, H. Zhang, and N. Abbasi (2007), An Experimental Investigation into the Feasibility of MIMO Techniques within the HF Band, paper presented at The Second European Conference on Antennas and Propagation, EUCAP, Edinburgh, UK.

Harrington, R. F. (1967), Matrix methods for field problems, Proceedings of the IEEE, 55(2), 149.

Jakes, W. C. (1974), Microwave Mobile Communications, Wiley, New York. 
Jenkins, R. W., L. E. Petrie (1997), Measurement and modelling of HF antenna gain and phase patterns and the effect on array performance, paper presented at Tenth international conference on antennas and propagation, Edinburgh, UK.

Kaye, A. R., D. A. George (1970), Transmission of Multiplexed PAM Signals Over Multiple Channel and Diversity Systems IEEE Transactions on Communication Technology, CO18(5), 520-526.

Kim, U. S., D. D. Lorenzo, J. Gautier, and P. Enge (2004), Phase effects analysis of patch antennas CRPAs for JPALS, paper presented at ION GNSS17th International Technical Meeting of the Satellite Division, Long Beach, CA, USA.

Lee, W. (1973), Effects on Correlation Between Two Mobile Radio Base-Station Antennas, IEEE Transactions on Communications, 21(11), 1214-1224.

Liang Dong, Hao Ling, and R. W. J. Heath (2002), Multiple-input multiple-output wireless communication systems using antenna pattern diversity, paper presented at IEEE Global Telecommunications Conference (GLOBECOM), Austin, USA.

Ling, J., D. Chizhik, P. Wolinansky, R. A. Valenzuela, N. Costa, and K. Huber (2002), MIMO measurement in Manhattan, paper presented at The 13th IEEE International Symposium on Personal, Indoor and Mobile Radio Communications, Lisbon, Portugal.

Loyka, S., G. Levin (2009), On physically-based normalization of MIMO channel matrices, IEEE Transactions on Wireless Communications, 8(3), 1107-1112.

Loyka, S. L. (2001), Channel capacity of MIMO architecture using the exponential correlation matrix, Communications Letters, IEEE, 5(9), 369-371. 
Malik, W. Q. (2008), Spatial correlation in ultrawideband channels, IEEE Transactions on Wireless Communications, 7(2), 604-610.

Martinsen, W. (2009), Giselle: A Mutually orthogonal triple twin-loop groundsymmetrical broadband receiving antenna for HF band, Command, Control, Communication and Intelligence Division, Defence, Science and Technology Organization, DSTO, Australia, TR-2321.

Massie, A., W. Martinsen, D. Taylor, M. Chamalaun, and J. Sorensen (2004), Giselle - DF using phase data from a ground symmetric loop, paper presented at Proceedings of the fifth Symposium on Radiolocation and Direction Finding, San Antonio, Texas, USA.

McNamara, L. F. (1991), The ionosphere: communications, surveillance and direction finding, Krieger publishing, Malabar, Florida.

Ng, K. H., E. K. Tameh, A. Doufexi, M. Hunukumbure, and A. R. Nix (2007), Efficient Multielement Ray Tracing With Site-Specific Comparisons Using Measured MIMO Channel Data, IEEE Transactions on Vehicular Technology, 56(3), 1019-1032.

Norton, K. A. (1936), The Propagation of Radio Waves over the Surface of the Earth and in the Upper Atmosphere, Proceedings of the Institute of Radio Engineers, 24(10), 1367-1387.

Paulraj, A., T. Kailath (1994), Increasing capacity in wireless broadcast systems using distributed transmission/directional reception (Patent No. 5345599). 
Paulraj, A., R. Nabar, and D. Gore (2003), Introduction to Space-Time Wireless Communication, Cambridge University Press, Cambridge, UK.

Peco, C., S. Zazo, I. Perez-Alvarez, and J. Lopez-Perez (2009), On the diversity in multiantenna HF communications, paper presented at 11th International Conference on Ionospheric radio Systems and Techniques, IRST, The Institution of Engineering and Technology, Edinburgh, U.K.

Perrine, C., Y. Erhel, D. Lemur, A. Bourdillon, and N. Melida (2006), 1300 km HF radio link with a $30 \mathrm{kbits} / \mathrm{s}$ data transfer rate, paper presented at 10 th IET International Conference on Ionospheric Radio Systems and Techniques, IRST, London, UK.

Salous, S., P. Filippidis, R. Lewenz, I. Hawkins, N. Razavi-Ghods, and M. Abdallah (2005), Parallel receiver channel sounder for spatial and MIMO characterisation of the mobile radio channel IEEE Proceed ings on Communications, 152(6), 912-918.

Shuzheng Xu, Hui Zhang, Huazhong Yang, and Hui Wang (2004), New considerations for high frequency communications, paper presented at 5th International Symposium on Multi-Dimensional Mobile Communications Proceed ings, China.

Strangeways, H. J. (2006), Estimation of signal correlation at spaced antennas for multi-moded ionospherically reflected signals and its effect on the capacity of SIMO and MIMO HF links, paper presented at 10th IET International Conference on Ionospheric Radio Systems and Techniques, London, UK.

Sung Ho Chae, Se-keun Oh, and Seong-Ook Park (2007), Analysis of Mutual Coupling, Correlations, and TARC in WiBro MIMO Array Antenna Antennas and Wireless Propagation Letters, IEEE, 6, 122-125. 
Svantesson, T., A. Ranheim (2001), Mutual coupling effects on the capacity of multielement antenna systems, paper presented at IEEE International Conference on Acoustics, Speech, and Signal Processing, (ICASSP '01).

Tarokh, V., H. Jafarkhani, and A. R. Calderbank (1999), Space-Time block codes from orthogonal designs IEEE Transactions on Information Theory, 45(5), 1456-1467.

Telatar, I. E. (1995), Capacity of multiantenna gaussian channels, European Transactions on Telecommunications, 10(6), 585-595.

Travers, D., J. Moore, and W. Sherrill (1965), Multiloop antenna arrays for highfrequency shipboard direction finding Antennas and Propagation, IEEE Transactions on Communications, 13(5), 665-671.

Van Wambeck, S. H., A. H. Ross (1951), Performance of Diversity Receiving Systems, Proceedings of the IRE, 39(3), 256-264.

Wallace, J. W., M. A. Jensen, A. L. Swindlehurst, and B. D. Jeffs (2003), Experimental characterization of the MIMO wireless channel: data acquisition and analysis, IEEE Transactions on Wireless Communications, 2(2), 335-343.

Winter, C. F. (1982), A note on utilizing far-field phase information, IEEE transactions on antennas and propagation, AP-30(5), 1010.

Winters, J. (1987), On the capacity of rad io communication systems with diversity in a rayleigh fading environment, IEEE Journal on Selected Areas in Communications, SAC-5, 878 . 
Zheng, I., D. Tse (2003), Diversity and multiplexing: A fundamental tradeoff in multiple antenna channels. IEEE Transactions on Information Theory, 49, 1073-1096. 


\section{Appendix: Published work}

N. Abbasi, E.M. Warrington, S.D. Gunashekar, S. Salous and S.M. Feeney, "HFMIMO capacity improvements using compact antenna arrays," Loughborough Antennas and Propagation Conference 2011, Loughborough, U.K.

N. Abbasi, "Antennas: The more the better," Festival of post graduate research, University of Leicester, U.K

S.D. Gunashekar, E.M. Warrington, S.M. Feeney, S. Salous, and N.M. Abbasi, "MIMO communications within the HF band using compact antenna arrays," Radio Sci., 45, RS 6013, doi. 10.1029/2010RS004416, 2010, pp1-16.

N. Abbasi, S.D. Gunashekar, E.M. Warrington, S. Salous, S. Feeney, L. Bertel, D. Lemur, M. Oger, "Capacity estimation of HF-MIMO systems", Ionospheric Radio Systems and Techniques, IRST 2009 pp 1-5.

S.D Gunashekar, E. M. Warrington, S. Salous, S. Feeney, N.M. Abbasi, L. Bertel, D. Lemur, M. Oger, 'Investigations into the feasibility of multiple input multiple output techniques within the HF band: preliminary results," Radio Science., vol. 44, RS0A19, 2009.

S.D Gunashekar, E.M. Warrington, H, Strangeways, Y. Erhel, S. Salous, S.M. Feeney, N. M. Abbasi, L. Bertel, D. Lemur, F. Marie, \& M. Oger, "Utilization of antenna arrays in HF systems," Annals of Geophysics 52 (3/4)(3/4): 323-338, 2009 
S.M. Feeney, S. Salous, E.M. Warrington, S.D Gunashekar, N. Abbasi, L. Bertel, D. Lemur \& M. Oger, "Compact antenna arrays for HF MIMO applications," Ionospheric Radio Systems and Techniques, IRST 2009, pp 1-5.

S.D. Gunashekar, E.M. Warrington, S. Salous, S. Feeney, N. Abbasi, D. Lemur, and M. Oger, " The use of heterogeneous antenna arrays in experimental HF-MIMO links," EUCAP 2009, pp 1-5.

S.D. Gunashekar, E.M. Warrington, N.M. Abbasi, S. Salous, S.M. Feeney, L. Bertel, D. Lemur and M. Oger, "HF MIMO communications using compact antenna arrays," UK URSI Festival of Radio Science, University of Birmingham, Birmingham, UK, December 2009.

S.M. Feeney, S. Salous, E.M. Warrington, S.D. Gunashekar, N.M. Abbasi, L. Bertel, D. Lemur and M. Oger, "Compact receive antenna arrays for HF MIMO applications," UK URSI Festival of Radio Science, University of Birmingham, Birmingham, UK, December 2009.

N.M. Abbasi, E.M. Warrington and S.D. Gunashekar, “Antennas Galore: Overcoming Bandwidth Limitations in High Frequency Radio Links," Festival of Postgraduate Research, University of Leicester, United Kingdom, June 2008.

S. Salous, E.M. Warrington, S.D. Gunashekar, S.M. Feeney, H. Zhang, N. Abbasi, L. Bertel, D. Lemur, M. Oger, "MIMO channel measurements in the HF band," URSI General Assembly, Chicago, 2008.

S. D. Gunashekar,, E.M. Warrington, S. Salous, S. Feeney, H. Zhang, N. Abbasi, L. Bertel, D. Lemur and M. Oger, "Early results of experiments to investigate the 
Feasibility of Employing MIMO Techniques in the HF Band," in Proceedings of the Loughborough Antennas and Propagation Conference, (March 2008, Loughborough, UK).

S.D. Gunashekar, E.M. Warrington, S. Salous, W. Kassem, L. Bertel, H. Zhang and N. Abbasi, "An Experimental Investigation into the Feasibility of MIMO Techniques Within the HF Band," IRI/COST 296 Workshop (Ionosphere - Modelling, Forcing and Telecommunications), Prague, Czech Republic, July 2007.

S.D. Gunashekar, E.M. Warrington, S. Salous, W. Kassem, L. Bertel, D. Lemur, H, Zhang, N. Abbasi, "An Experimental Investigation into the Feasibility of MIMO Techniques within the HF Band,” EUCAP 2007, Edinburgh, U.K. 